\title{
IMPLEMENTATION OF PHYSIOLOGIC FLOW CONDITIONS IN \\ A BLOOD VESSEL MIMIC BIOREACTOR SYSTEM FOR \\ THE EVALUATION OF INTRAVASCULAR DEVICES
}

\author{
A Thesis \\ Presented to the Faculty of \\ California Polytechnic State University, San Luis Obispo
}

\author{
In Partial Fulfillment \\ of the Requirements for the Degree \\ Master of Science in Engineering \\ with a Specialization in Biomedical Engineering
}

by Marc Cody Dawson

April 2009 
(C) 2008 Marc C. Dawson

ALL RIGHTS RESERVED 


\section{THESIS COMMITTEE MEMBERSHIP}

Title: IMPLEMENTATION OF PHYSIOLOGIC FLOW CONDITIONS

IN A BLOOD VESSEL MIMIC BIOREACTOR SYSTEM

FOR THE EVALUATION OF INTRAVASCULAR DEVICES

Author: Marc C. Dawson

Submitted: April, 2009

Committee Chair: Dr. Kristen O'Halloran Cardinal

Committee Member: Dr. Trevor Cardinal

Committee Member: Dr. Scott Hazelwood 


\author{
ABSTRACT \\ IMPLEMENTATION OF PHYSIOLOGIC FLOW CONDITIONS IN \\ A BLOOD VESSEL MIMIC BIOREACTOR SYSTEM FOR \\ THE EVALUATION OF INTRAVASCULAR DEVICES \\ by \\ Marc C. Dawson
}

The prevalence and devastating nature of cardiovascular diseases has led to many advancements in the therapies used to treat the millions of patients that suffer as a result of these conditions. As coronary artery disease (CAD) is the most common of these cardiovascular conditions, it is a major focus of research among the medical industry. Although lifestyle changes and drug therapies can treat early CAD, more advanced cases often require more definitive interventions. In conjunction with angioplasty, stenting of an occluded vessel has shown significant success in preventing restenosis. However, as with nearly every therapeutic process in the medical field, several complications have arisen in stented patients that pose a need for further improvement of the devices. As a result, the stent industry is constantly striving towards improving the characteristics and outcome of their product and with these efforts comes the need for extensive testing and research.

Continuous improvement and innovation in the field of tissue engineering has brought about the possibility of creating laboratory grown tissue engineered vascular grafts (TEVGs) for the purpose of replacing and/or bypassing damaged or occluded 
regions of the vasculature. By employing the techniques used to produce TEVGs, a blood vessel mimic (BVM) bioreactor system has been developed with the intent of using the resulting construct as a model for testing the cellular response of a human blood vessel to an intravascular device such as a stent. This would allow gathering of more significant data in the early stages of device development and may reduce the overall costs and time required to refine a design.

Although the BVM system has previously been used to cultivate viable constructs that were subsequently used to observe the response to a deployed stent, the flow conditions within the original design are not representative of the physiologic conditions in a native vessel. This aspect of the original system presented a need for development in order to be considered by researchers as an accurate in vitro representation of the target vessels in which the stents are used. One of the primary concerns of this environment is creating and maintaining physiologic flow conditions that will represent those present in native vessels in order to facilitate cells sodded on the construct to grow as they would under native conditions. The two key aspects of flow are pulsatility and wall shear stress.

Studies in this thesis were carried out to determine the best and most feasible methods for implementing appropriate levels of pulsation and wall shear stress in the previously established BVM bioreactor system with the intention of maintaining the original system's simplicity and high throughput potential. Pulsatile flow was created by elevating backpressure in the BVM chamber while using a different pump head and pump tubing. Wall shear stress was adjusted by altering the viscosity of the perfusate and flow rate through the system. Both pulsatile flow and shear stress were established without any major changes to the overall configuration of the system. 
Pulsatile pressures of $\sim 80 \mathrm{mmHg}$ and wall shear stress forces of $\sim 6.4 \mathrm{dyn} / \mathrm{cm}^{2}$ were established with minimal alteration to the original system. Pulsatility was created by using a 3-roller peristaltic pump head in place of the originally specified 8-roller head to create pulses that were then regulated with backpressure created by restricting down stream flow. Increasing the viscosity and corresponding flow rate allowed for instigation and control of wall shear stress at the inner wall of the BVM graft. Although the resulting protocols presented here require refinement for ultimately successful implementation, they are important underpinnings that will facilitate the eventual development of an ideal BVM system that is highly suitable for use as a high-throughput intravascular device testing model. 


\section{ACKNOWLEDGEMNTS}

Firstly, I would like to thank Dr. Kristen O'Halloran Cardinal for her continuous care and support of my efforts to complete my endeavors and for the opportunity to be a part of a team working towards a system that has the potential to greatly contribute to medical device development.

Additionally, I would like to thank committee members Dr. Trevor Cardinal and Dr. Scott Hazelwood for their input and cooperation into the betterment of this thesis.

I would also like to express my gratitude to Hans Mayer, M.S. and Dr. David Clague for improving my understanding of fluidics along with their contributions that allowed me to complete this undertaking.

My thanks and love go out to my wife, Sarah Kim and my parents, David and Cydney for their mental and emotional support through the entirety of my degree and the completion of my thesis. You all made it possible for me to achieve my goal with success and satisfaction. I know it was hard for all of us, but you have helped me more than you know and helped me get to the place where I can move on and enjoy the spoils.

I'd also like to give a shout out to my fellow members of the Cal Poly Tissue Engineering Laboratory O.G. members, Dimitri Delagrammaticas and Colby James (listed in alphabetical order). Thanks for all of the help and assistance. It was great working with you guys. To think that 41-209 was without us for so many years. See http://bmegene.calpoly.edu/facilities/te/ for our immortal imprint and fame on the world wide web. 


\section{TABLE OF CONTENTS}

INDEX OF TABLES

INDEX OF FIGURES

xii

CHAPTER 1: INTRODUCTION

OVERVIEW

PHYSIOLOGY AND PATHOLOGY OF VASCULATURE

Cadiovascular System 2

Clinical Pathologies $\ldots$

CAD Treatment Options

PRECLINICAL TESTING OF INTRAVASCULAR DEVICES

Tissue Engineered Blood Vessels as preclinical device testing models _................ 11

Financial Concerns

BVM as a Testing Environment

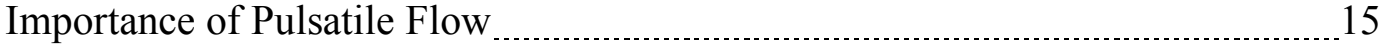

Importance of Wall Shear Stress 17

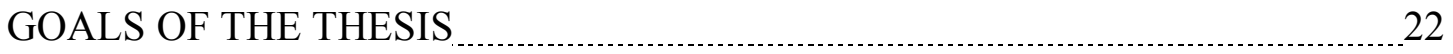

HYPOTHESIS

CHAPTER 2: DEVELOPMENT OF PULSATILE FLOW SYSTEM

INTRODUCTION 25

MATERIALS AND METHODS

Pump Head and Tubing Selection …….......................................................... 29

Actual Flow Rate vs. Pump Speed $\quad 30$

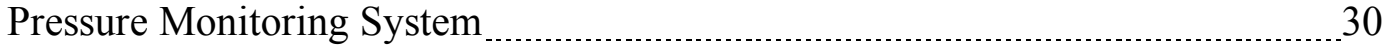

Characterization of Original Steady Flow BVM System $\quad 31$

Characterization and Development of the Pulsatile Flow Setup _....................... 32

Development of Blood Pressure Model Settings _.................................................. 35

Preliminary Cell Adhesion Test 36

Pressure Testing with First and Second Generation BVM Chambers _................... 37

Final Cell Adhesion Tests _................................................................................... 38 


\section{RESULTS}

Estimating Flow Rates

Actual Flow Rate vs. Pump Speed 43

Pressure Fluctuation Tests

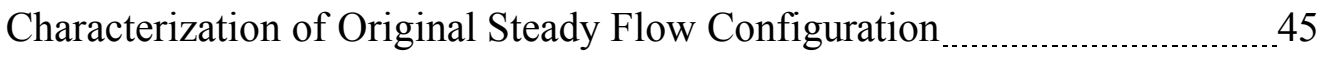

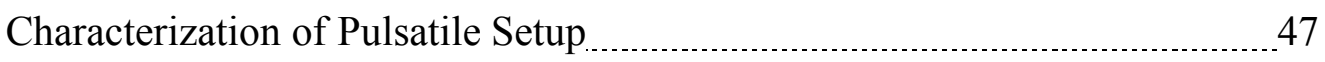

Initial Testing with Closed Loop _ 47

Addition of BVM Chamber

System Pressure with Water vs. Media 48

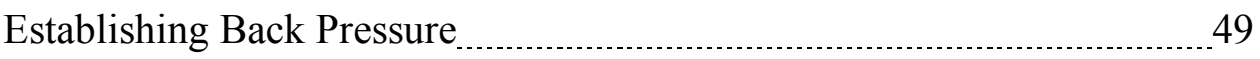

Pressure Differentials: Cardiac vs. BVM

Preliminary Cell Adhesion Test 55

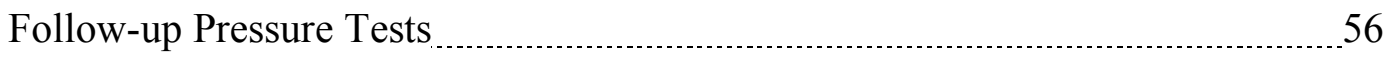

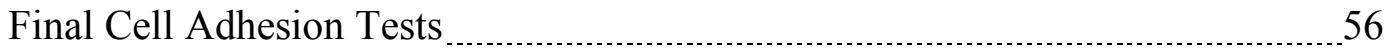

DISCUSSION

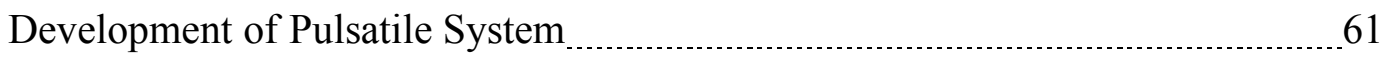

Scalability 63

Comparison to Previous Work

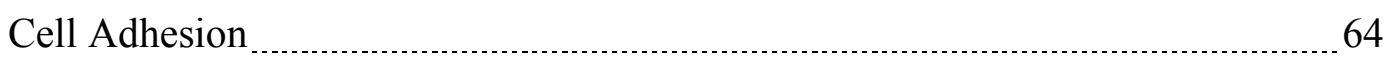

Construct Appearance and Cell Morphology 65

\section{CHAPTER 3: ESTABLISHING AND CONTROLLING WALL SHEAR STRESS INTRODUCTION}

Physics of Shear Stress in a Tube 68

Effects of Shear Stress on Blood Vessel Physiology 69

Importance of and Minimum Shear Stress Required for Cell Alignment _............. 72

Previously Used Methods for Implementing WSS in TEVGs 73

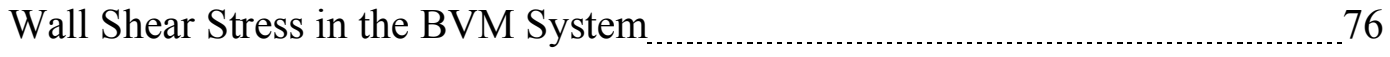

MATERIALS AND METHODS

Determination of Necessary Viscosity

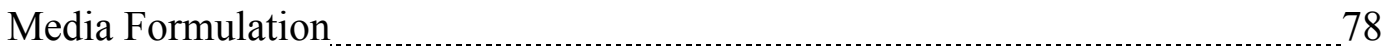


Viscosity Testing

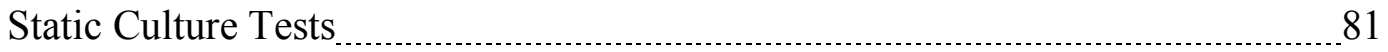

Cell Adhesion Tests

\section{RESULTS}

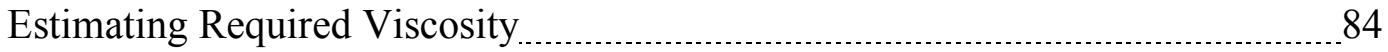

Viscosity Testing on Vilastic-3 Viscometer

Characterization of Media Viscosity $\quad 87$

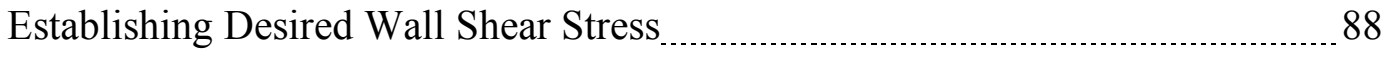

Static Culture Tests 89

Cell Adhesion Tests

DISCUSSION

Development of Wall Shear Stress 98

Effect of Dextran on HUVEC Cultures

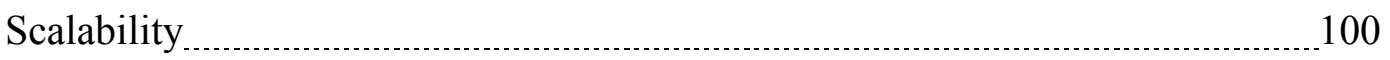

Comparison to Previous Work 100

Cell Adhesion

Construct Appearance and Cell Morphology 102

\section{CHAPTER 4: DISCUSION AND CONCLUSIONS}

OVERVIEW

FUTURE WORK

Pulsatile Flow 105

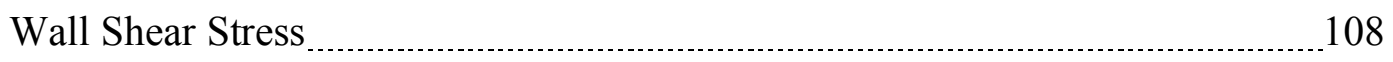

Cell Culturing $\quad 110$

Additional Future Work and Considerations $\quad 112$

CONCLUSION

CITED REFERENCES

APPENDIX A: Additional Figures

APPENDIX B: Procedural Protocols $\ldots \ldots \ldots$

APPENDIX C: Trial Construct Location $\ldots$

APPENDIX D: Figure Reprint Permissions 142 


\section{INDEX OF TABLES}

Table 2.1 Outline of tests performed using AD Instruments Data Acquisition System...31

Table 2.2 Target pressures for BVM Blood Pressure Models [80] ...................................36

Table 2.3 Pulsatile flow cell adhesion test condition summary.....................................39

Table 2.4 Data for initial comparison of potential pump configurations........................ 41

Table 2.5 Flow rates estimated using linear equations extrapolated from manufacturer's data. Blue highlighted flow rates correspond to those specified in the configuration in the original system. ......................................................... 42

Table 2.6 Actual flow rates of steady and pulsatile flow configurations.........................43

Table 2.7 Measured flow characteristics of steady flow configuration. ...........................46

Table 2.8 Measured flow characteristics of complete pulsatile system with ratchet

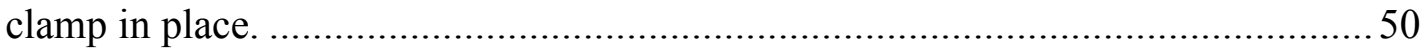

Table 2.9 Comparison of cardiac blood pressure conditions and flow settings in pulsatile BVM system (blood pressure figures taken from [80].............................53

Table 3.1 Shear stress cell adhesion test condition summary........................................ 83

Table 3.2 Calculated shear stress in BVM with $4 \mathrm{~mm}$ graft at 16-24 $\mathrm{ml} / \mathrm{min} \ldots \ldots \ldots \ldots \ldots \ldots . . .85$

Table 3.3 Calculated shear stress in BVM with $4 \mathrm{~mm}$ graft at $25-33 \mathrm{ml} / \mathrm{min} \ldots \ldots \ldots \ldots \ldots \ldots . . .85$

Table 3.4 Calculated shear stress in BVM with $4 \mathrm{~mm}$ graft at 34-40 $\mathrm{ml} / \mathrm{min} \ldots \ldots \ldots \ldots \ldots \ldots . . .85$

Table 3.5 Viscosity of lower and higher molecular weight dextran in complete

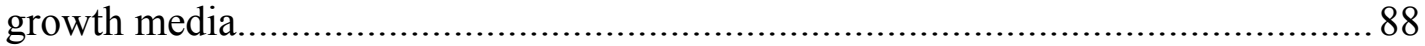

Table 3.6 Estimated confluence of static cultures of HUVECs grown in bioreactor

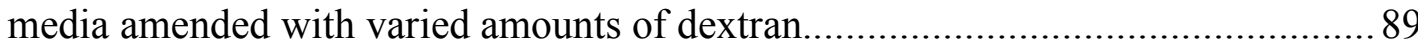




\section{INDEX OF FIGURES}

Figure 1.1 Basic structures of human artery and vein. 3

Figure 1.2 Plaque depositions in atherosclerotic artery. 5

Figure 1.3 Angioplasty with deployment of intravascular stent. 7

Figure 1.4 Pressure waveforms of steady flow BVM and native arteries. 14

Figure 1.5 Flow analysis of arterial bifurcation. 21

Figure 2.1 Pulsatile flow bioreactor by Hahn et al. 27

Figure 2.2 Diagram of bioreactor system by Hoerstrup et al. 28

Figure 2.3 Placement of pressure transducer in steady and pulsatile configurations. .......32

Figure 2.4 Steady flow configuration set up for pressure characterization. 33

Figure 2.5 Pulsatile flow configuration set up for pressure characterization. 35

Figure 2.6 Removal of ePTFE graft from BVM bioreactor chamber. 40

Figure 2.7 Plot of manufacturers estimated flow rates with linear approximations. 41

Figure 2.8 Plot of estimated flow rates using different pump tubing diameters. 42

Figure 2.9 Plot of actual flow rates of 3 and 8 roller pump heads in Table 2.6. 44

Figure 2.10 Estimated vs. actual flow rates using 3-roller pump head. 44

Figure 2.11 Pressure profile of steady flow setup at $\sim 11 \mathrm{ml} / \mathrm{min}$. 45

Figure 2.12 Plot flow characteristics of steady flow setup with 8-roller pump head. ....... 46

Figure 2.13 Pressure profiles of closed loop with 3-roller pump at $\sim 18.5 \mathrm{ml} / \mathrm{min}$. 47

Figure 2.14 Pressure profile of system with 3-roller pump at $\sim 18.5 \mathrm{ml} / \mathrm{min}$. 48

Figure 2.15 Comparison of perfusion fluids in pulsatile system at $\sim 18.5 \mathrm{ml} / \mathrm{min}$. 49

Figure 2.16 Tested options for creating backpressure. 50

Figure 2.17 Plot of flow characteristics of pulsatile setup from data in Table 2.8. 51 
Figure 2.18 Pressure profiles with constant backpressure and varied pump speed.

Figure 2.19 Comparison of BVM vs. native cardiac pressures.

Figure 2.20 Comparison of BVM vs. native cardiac pressure differentials. 54

Figure 2.21 Explanation of construct naming scheme. 57

Figure 2.22 BBI(A) and SEM(B) imaging of construct PA1. 59

Figure 2.23 BBI(A) and SEM(B) imaging of construct PB1. 59

Figure 2.24 BBI(A) and SEM(B) imaging of construct PB2. 60

Figure 2.25 BBI(A) and SEM(B) imaging of construct PB5. 61

Figure 2.26 BBI(A) and SEM(B) images of ePTFE graft sodded with HMVECs. 66

Figure 2.27 SEM images of HUVECs cultivated in a perfusion bioreactor system on a polyurethane graft. 66

Figure 3.1 Vilastic-3 Viscometer system. 81

Figure 3.2 Viscosity and shear stress of increasing Dextran concentrations. 86

Figure 3.3 Shear stress in BVM with varied media Dextran concentrations. 87

Figure 3.4 Growth media as a Newtonian fluid. 88

Figure 3.5 HUVECs static culture -Day 0. 91

Figure 3.6 HUVECs static culture-Day 1,3 and 5. 92

Figure 3.7 BBI(A) and SEM(B) imaging of construct SA1. 94

Figure $3.8 \mathrm{BBI}(\mathrm{A})$ and $\mathrm{SEM}(\mathrm{B})$ imaging of construct SA2. 95

Figure 3.9 BBI(A) and SEM(B) imaging of construct SB1. 96

Figure $3.10 \mathrm{BBI}(\mathrm{A})$ and SEM(B) imaging of construct SB2. 96

Figure $3.11 \mathrm{BBI}(\mathrm{A})$ and SEM(B) imaging of construct SB3. 97

Figure 3.12 BBI(A) and SEM(B) images of ePTFE graft sodded with HMVECs. 102

Figure A.1 BVM bioreactors in incubator for wall shear stress cell adhesion trial. 123 
Figure A.2 BVM bioreactors in incubator setup for pulsatile flow cell adhesion trial....124

Figure A.3 SEM image of construct PB1. 125

Figure A.4 High magnification SEM image of HUVEC on construct PB2. 125

Figure A.5 BBI image of construct PB3. 126

Figure A.6 SEM image of construct PB3. 126

Figure A.7 BBI image of construct PB4. 127

Figure A.8 SEM image of construct PB4. 127

Figure A.9 Higher magnification SEM image of construct PB4. 128

Figure A.10 Higher magnification SEM image of construct PB5. 128

Figure A.11 Higher magnification SEM image of construct SA1. 129

Figure A.12 Higher magnification SEM image of construct SB2. 129

Figure A.13 BBI image of construct SA3. 130

Figure A.14 SEM image of construct SA3. 130 


\section{Chapter 1: INTRODUCTION}

\section{OVERVIEW}

Along with many other laboratories around the globe, our group is attempting to develop an efficient, simple bioreactor system that can be used to grow human blood vessels in vitro. The primary goal of nearly every other group is to produce a Tissue Engineered Vascular Graft (TEVG) that can be implanted in human patients who are in need of a replacement for vessels that have been rendered dysfunctional by disease or trauma $[29,33,50,58]$. Although our lab aims to meet similar criteria by following the same principles of tissue engineering, our Blood Vessel Mimic (BVM) system is in development for the purpose of creating an in vitro assay for intravascular devices or drugs that will provide in vivo-like results.

Creating a viable BVM that has the qualities of a native vessel could provide a means to test intravascular devices or drugs that could reduce the cost and danger involved in preclinical and clinical trials that rely on animal and human testing. This system could prove to be very desirable to the medical community and device industry as it would be a valuable tool that could be implemented early in the extensive development process.

In its current state, our system has several shortcomings that must be addressed. This paper will explore the flow conditions in the system along with the formulation of the growth media with the purpose of establishing pulsatile flow and physiologic wall shear stress to promote and improve the development of the vessel. In order to fully understand the topic and the research that will be presented here, the physiology and 
pathology of the cardiovascular $(\mathrm{CV})$ system as well as the current practices, treatment methods, and state of TEVG research will be reviewed.

\section{Physiology and Pathology of Vasculature}

The Cardiovascular System

The cardiovascular system is comprised of three major components: blood vessels, which serve as conduits, the heart, which functions as a pump with the primary role of transporting nutrients and oxygen-carrying red blood cells throughout the body and finally, blood, the carrier. The two main vessel types, arteries and veins, transport oxygen-rich blood to the body's organs and oxygen-deprived blood back to the lungs. Although their primary function is to distribute blood throughout the body, blood vessels are not simple tubes of tissue, but rather complex structures designed to regulate blood pressure, oxygen and nutrient distribution, as well as to initiate and transmit chemical and neuronal signals in an effort to maintain and control cardiovascular function [73]. The coronary arteries supply blood to the heart and are of particular importance to this thesis as they are the target for devices that we aim to evaluate.

There are three layers that comprise the vessels of the body (Figure 1.1). The innermost and thinnest layer, known as the tunica intima, lines the interior of the cardiovascular system and is continuous throughout the vasculature and heart, existing as an antithrombogenic surface that directly contacts whole blood. The intima, also known as the endothelium, is primarily made up of endothelial cells (ECs) backed by a thin basement layer of connective tissue. In some muscular arteries and arterioles, the ECs are attached to an internal lamina layer. The middle layer, the tunica media, is primarily 
composed of smooth muscle cells (SMCs) supported by a layer of elastic fibers that provide mechanical strength. The SMCs aid in blood pressure regulation via radial contraction/relaxation of the vessels; an action that is controlled by a network of nerves that stem throughout the medial layer from the autonomic nervous system.

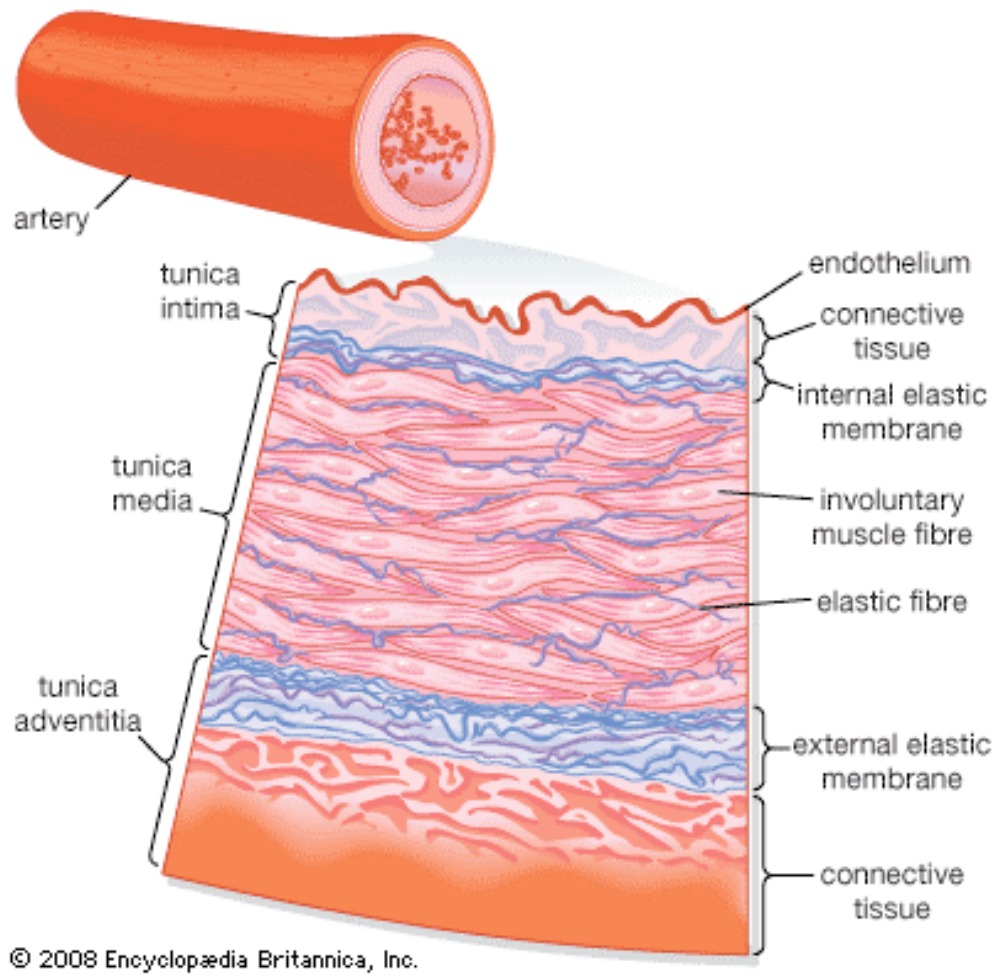

Figure 1.1 Basic structure of human blodd vessels [4]. (Permission to reprint figure granted by Encyclopedia Britanica. See Appendix D)

The outermost and thickest layer, known as the tunica adventia, encapsulates the vessel to provide overall strength and appropriate compliance with varying blood pressures. The adventia is primarily composed of collagen and elastic fibers. In thicker vessels, a microvascular network known as the vaso-vasorum passes through the adventitia to supply the medial layer with blood. However, in thinner vessels, this process occurs via 
diffusion. The nerves that control the SMCs of the medial layer also pass through the adventitia.

\section{Clinical Pathologies}

Complications and diseases of the cardiovascular system, specifically of the blood vessels, are a leading cause of death and disability in the United States and the rest of the world, resulting in nearly 870,000 deaths in 2005 in the U.S. alone [5]. Coronary artery disease $(\mathrm{CAD})$ is the most common of these ailments leading to more than half of these deaths [5]. Although preventative measures such as lifestyle modification or drug intervention can reduce the chances of developing these conditions, their presence often goes undetected for too long and damage to the vessels is irreparable.

An underlying condition that gives rise to many $\mathrm{CV}$ diseases and disabilities is arteriosclerosis. This condition is a result of damage, stiffening and subsequent inflammation of the endothelial layer of blood vessels or heart and often results in a restriction of blood flow to tissues and/or organs. The major type of arteriosclerosis is atherosclerosis, in which atheromas (plaques of fatty deposits) form on the inner walls of arteries (Figure 1.2) [6,49,77]. 


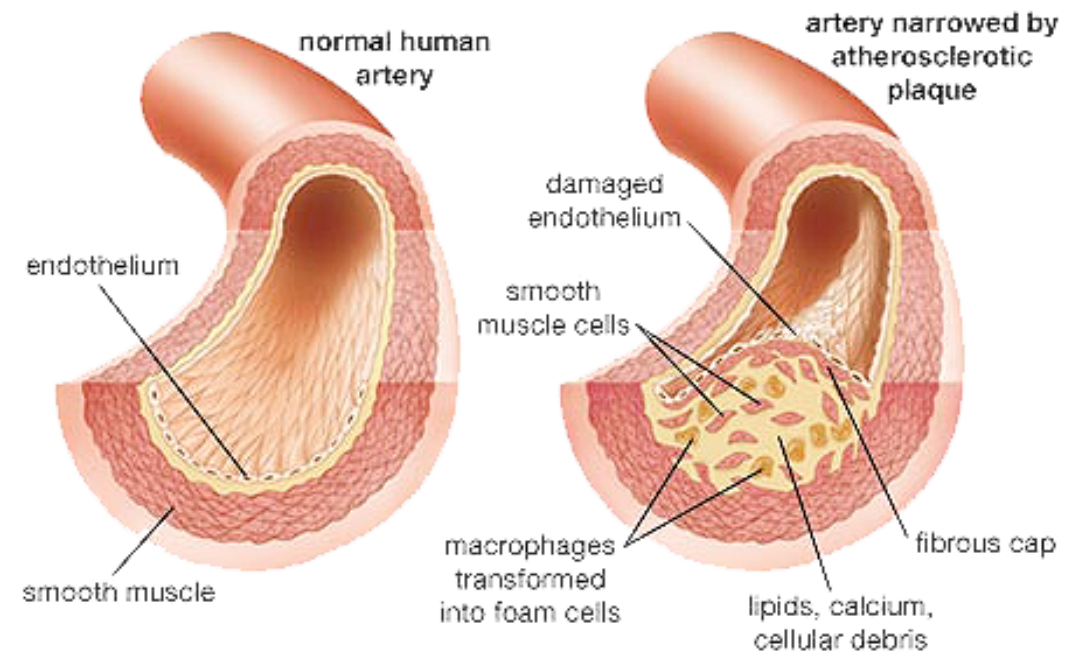

Figure 1.2 Plaque deposition in atherosclerotic artery [4]. (Permission to reprint Figure granted by Encyclopedia Brittannica. See Appendix D)

Atherosclerosis is progressive and typically begins in all people, to some degree, at birth $[6,49,77]$. The result is a hardening and/or narrowing of the arteries due to thickened arterial walls or plaque formation. Plaque lesions form on the endothelial layer usually as a result of lifestyle (high fat diet, low activity level, smoking, etc.) or genetic predisposition and if not addressed, may proceed to grow inward, reducing the size of the vessel lumen possibly resulting in vessel occlusion [6]. There are two common types of plaques: hard stable and soft unstable. The hard and stable type often cause luminal reduction, where as the soft and unstable type are more likely to break free from the vessel wall, causing a flow-impeding clot downstream that may result in organ damage or death. Further complications that can result from impeded blood flow or released clots are myocardial infarction (MI), ischemic cardiomyopathy, ischemic and hemorrhagic stroke and peripheral artery disease $[49,77]$. Vessel occlusion can be treated with drugs or interventions such as angioplasty or stenting as discussed below. 


\section{CAD Treatment Options}

When treatment beyond drug therapy and lifestyle adjustments is required for patients with $\mathrm{CAD}$, there are several options including coronary revascularization (or coronary bypass grafting - CABG), coronary angioplasty, and coronary stenting. CABG involves the use of a portion of a healthy vessel from the patient's chest or leg to redirect blood around the lesion site(s) restoring proper flow [7]. This is a highly invasive procedure that requires opening the chest cavity and a recovery period of 4-8 weeks [78].

Angioplasty, a less invasive option, involves the insertion of a catheter equipped with an inflatable balloon into the occluded vessel (Figure 1.3). The balloon portion of the catheter is positioned at the site of occlusion where it is then inflated in hopes that the occlusion will be forced open, restoring proper blood flow. According to the American Heart Association's 2008 Update to Heart Disease and Stroke Statistics Report, in 2005 there were over 1,250,000 angioplasties performed in the United States [8]. It is estimated that nearly $95 \%$ of all angioplasties (with or without stenting) performed in the United States annually have initially successful outcomes, where success is defined as prevention of death or the need for an emergency CABG [65]. Stenting, which involves the deployment of a tubular mesh structure in an occluded vessel, has improved this success rate by reducing the need for emergency CABG from $\sim 6 \%$ to less than $1 \%$ [87]. 


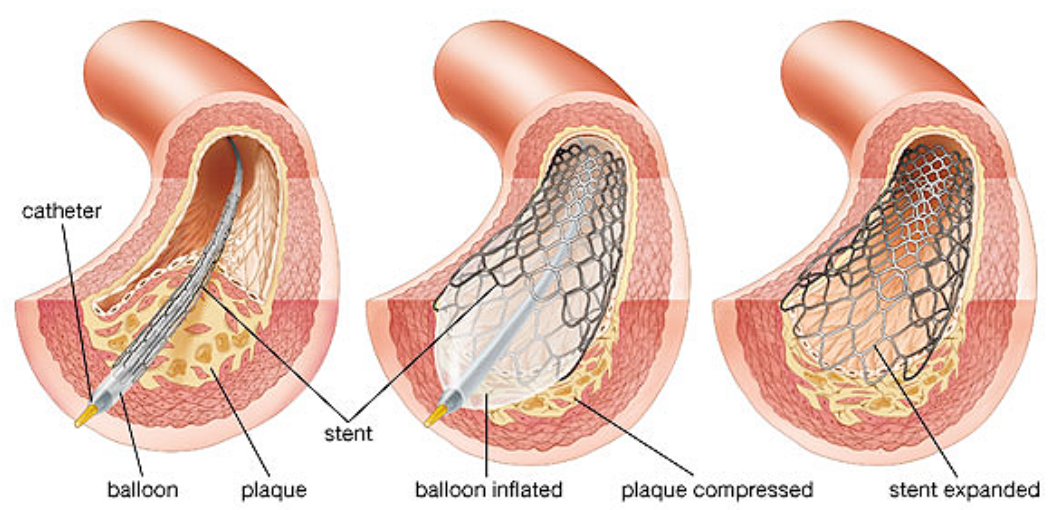

Figure 1.3 Angioplasty with deployment of intravascular stent [3]. (Permission to reprint Figure granted by Encyclopedia Brittannica. See Appendix D)

The FDA originally approved coronary stents for use when the results of angioplasty were suboptimal [87]. Their use has since increased with approximately $91 \%$ of angioplasties performed in 2007 including the placement of a coronary stent to hold the vessel open, thus reducing the chance of restenosis and the need for a repeated procedure [8].

Commercially available stents are commonly of two types: bare metal and drug eluting. Stents consist of a structural cage made of specific alloys such as $316 \mathrm{~L}$ stainless steel and cobalt chromium that are designed to be as biocompatible as possible so that when implanted the metal from which they are made is accepted by the body [44]. As with most implantable devices, there is a necessary balance between preventing rejection and promoting some degree of tissue integration while avoiding tissue over-growth. In the case of coronary stenting, this integration occurs in two processes: neointimal generation and endothelial regeneration [27]. Neointimal generation, which is typically considered a problematic response, involves an initial inflammatory response followed by an influx of lymphocytes and then SMCs around the struts of the stent in as little as 72 hours [27,66]. Endothelial regeneration, which is typically considered a desirable 
response, includes the influx of Factor VIII positive endothelial cells with complete coverage occurring in about 96 days in humans who have received a Bare Metal Stent (BMS) [27]. Extensive cell proliferation or neointimal hyperplasia in response to the metal stent itself can result in restenosis of the vessel and a negation of the effects of the procedure [52]. This has been a major complication in vascular stenting occurring in up to $80 \%$ of patients at 6 months post procedure and has lead to the development of drug eluting stents (DESs) [52,71].

Drug eluting stents are structurally similar to bare metal stents but are often coated with a polymer that is infused with an anti-inflammatory drug such as paclitaxel, dexamethasone or the limus drugs [52,72]. Cytostatic compounds such as these were thought to be the solution to neointimal hyperplasia by preventing cell replication at the implantation/injury site. However, several new issues have arisen that have inhibited the success of these devices $[52,71,72]$. Because these compounds reduce cell proliferation, they consequently inhibit healing at the site as well as proper cellular integration of the stent itself and can lead to coronary thrombosis [52]. Additional issues regarding the success of DESs include achieving proper drug concentration and potency, biocompatibility of the polymer coating, and maintaining long term efficacy of the drug $[52,71,72]$. Controlling this has been the target of many studies and due to the complex nature of cellular growth, has yet to be perfected $[71,72]$.

As discussed above, a stent has not yet been developed that is entirely effective at consistently preventing restenosis while achieving proper tissue integration. For this purpose, an in vitro tissue engineered BVM system could be an ideal testing environment as it would allow the study of the human tissue response to different bare metal and DES 
configurations that could reduce the cost and danger of animal and human studies. Due to the importance of preclinical testing for newly emerging stents and other intravascular devices, the following section will briefly review currently employed preclinical procedures.

\section{Preclinical Testing of Intravascular Devices}

The invasiveness and life supporting nature of intravascular devices requires that they undergo extensive testing prior to approval for use in people [35]. Although the final step in this process is ensuring safety and efficacy through clinical trials, a process that can take years and cost millions of dollars, the preclinical trials are also extensive, time consuming, and costly. Preclinical trials include bench, in vitro and animal testing, and typically aim to show that the device's design is appropriate for a given application and will not be toxic or damaging to the person receiving it. These preclinical studies can take 2-3 years and cost $\$ 10-20$ million dollars [35,53]. Once a device passes this step, it is subject to approval for use in clinical trials in humans. This approval process in and of itself can take three to six months [35]. Following evaluation of the clinical trials, devices can potentially be approved for market.

Many preclinical testing methods have been developed that provide an estimation of the human body's response to a device. Bench and in vitro testing include biochemical assays that assess biocompatibility, cytotoxicity and the potential for thrombogenicity, as well as mechanical testing that determines if the structural characteristics of the device are appropriate for its intended use [53]. For example, DESs have been tested using in vitro cell cultures to determine the effect of dexamethasone on SMCs and in porcine 
animal models to observe the development of neointimal hyperplasia in response to implantation of a sirolimus-eluting stent $[38,63,88]$. Although these methods are good approximations, there is still a need for more physiologic and more cost effective methods, respectively.

Animal testing involves implanting the device in an appropriate lab animal and evaluating the tissue response to the device $[38,88]$. When testing a coronary stent for example, the stent is implanted in the iliac or coronary artery of an animal (often a rabbit or a pig) using similar methods as would be used on a human patient. Depending on the study, the artery containing the stent is excised at specified time points and analyzed for responses including platelet and fibrin deposition, infiltration by blood and vascular cells and the formation of extracellular matrix (ECM) and collagen fibrils [81]. While animal testing is one of the gold standards in device development and allows for the examination of a device in vivo, in some cases, such as endovascular stenting, it has proven to be a poor example of what will occur when the device is introduced in a human subject [81]. In the case of stents, it has been shown that an animal model can predict responses to stenting but the demonstrated time period is typically much shorter than in humans [81]. This may be due to the fact that stents implanted in test animals are placed in clean, lesion free vessels rather than damaged, sclerotic ones [81]. Another possibility is that the healing time and thus the tissue response rate is proportional to the lifespan of the animal [81]. An animal with a shorter lifespan may have a faster healing rate leading to inaccurate results when predicting time and longevity of a device in humans.

The next step following successful animal studies is to determine the acceptance of and response to the device in human subjects through clinical trials [27,59]. Although 
preclinical trials can help in determining whether a device will be accepted by or cause major complications in the body, the device may fail clinical trials making the extensive time and money expended to bring a device to this point in development lost. This is often the case with many devices [35] and it would be beneficial if there were a way of testing the device in appropriate human tissues before getting to the point of clinical trials. Tissue engineering could be employed to create a sample tissue that could be used for this purpose.

\section{Tissue Engineered Blood Vessels as preclinical device testing models}

For several years, great efforts have been made to produce tissue engineered vascular grafts with the intent of implanting them into patients needing bypasses or vascular repair who cannot provide an autologous graft $[29,33,50,58,64,85]$. These efforts are of considerable merit and could be of great benefit to a vast patient base [5]. Using similarly produced grafts as living vessel models in preclinical testing of novel intravascular devices could provide value by decreasing the time and cost of development and approval of the many valuable devices that can benefit thousands of people.

Until an ideal device is developed and made readily available, there will always be efforts towards continuous improvement of current devices. Each measure of improvement, regardless of the degree of change, will be subjected to testing and approval and thus great expenditures and scrutiny by regulatory bodies. That said, an effective, accurate in vitro model could reduce initial testing costs and improve final success rates for the industry while providing data to regulatory agencies to back the safety and efficacy of these devices. 


\section{$\underline{\text { Financial Concerns }}$}

Assuming that the BVM system can be developed to produce tissue that is very similar to native vessels, not only would it be desirable to device companies for its early indicator capabilities, but it could also give valuable data at a fraction of the cost of animal testing. For the purpose of intravascular devices, rabbits are the preferred animal used in initial animal testing for the size and similarity of their iliac arteries as compared to human coronary arteries. For example, there are no functional stents that are small enough to implant in mice. Although it is difficult to attain exact figures regarding the total cost of testing coronary stents in animals, as it varies greatly with device and research group, the costs of the actual lab animals were available from Charles River Laboratories [15]. Disregarding size and sex, the least expensive rabbit available from Charles River is $\$ 84.55$. When size and sex are of importance, costs can reach as much as $\$ 183.55$ per rabbit.

For chronic studies, a major US vascular device company uses United States Department of Agriculture purpose bred dogs that cost $\sim \$ 3000$ per animal [16]. These costs are for the animal only and do not include housing, surgery and maintenance costs. Equipping a facility for survival surgeries and maintenance of up to 200 test animals can easily reach $\$ 1.3$ million [16]. If a device company performs the testing in-house, the cost of consumables used in a survival surgery alone are approximately $\$ 1000$ per animal and maintaining that animal in an IACUC approved facility before and after surgery costs

around \$20 per day, per animal [16]. Additionally, staffing the facility with a veterinarian and two technicians can cost an estimated $\$ 240,000$ per year. Excluding 
surgery costs, when taking all expenditures into account, the annual cost for maintaining an animal testing facility with 200 animals on hand approaches $\$ 2$ million per year [16].

In comparison, the current generation of BVM bioreactor system is approximately $\$ 15.25$ for the growth chamber, tubing and media reservoir. This does not include the cost of incubators, pumps, media solutions, scaffold materials and cell cultures, however several of these costs are incurred only upon initial setup of the testing facility as they are reusable equipment. The initial cost required to equip a laboratory with the necessary hardware to produce up to thirty-two steady flow or sixteen pulsatile flow systems, is approximately $\$ 47,000$. Once the testing facility is established, excluding labor costs, the only cost is consumables, which amounts to approximately $\$ 100$ for each graft setup.

The financial comparison discussed above is not meant to show that the millions of dollars spent on animal testing can be saved by replacing animal testing with our device, but rather that our device can help improve the triage process when deciding when and what devices should proceed to animal testing. When used in this way, there would be a great potential savings in testing costs as our system could be used to eliminate faulty designs early in the development process before placing it in animals.

Along with the financial concerns, use of our system in the development process could affect IACUC decisions and ethical dilemmas surrounding animal testing. Not only would unnecessary animal testing be reduced, but also animal studies could actually be improved as the decision on the number of animals to use could be adjusted based on data gathered from our system. 


\section{$\underline{B V M}$ as a Testing Environment}

A novel bioreactor system has been developed by our laboratory that has potential for use in intravascular device testing by creating a Blood Vessel Mimic (BVM) that can be grown in vitro for the purpose of deploying a stent and studying the human cellular response to the device [12]. However, in its current state, the environment in which the graft is grown is not truly representative of a physiologic vessel environment.

The luminal flow through the vessel lacks pressure fluctuations that would represent cardiac output (See Figure 1.4. Note difference in scale of Y-axes) and the viscosity of the media is well below that which is required for proper shear stress and cell alignment. The shear stress in native arteries is $>15 \mathrm{dyne} / \mathrm{cm}^{2}$ and the minimum required for proper EC alignment is $6-10$ dyne $/ \mathrm{cm}^{2}[25,28,42,46]$. The shear stress produced by the media in our original steady flow system is $\sim 0.359$ dyne $/ \mathrm{cm}^{2}$ when using a $4 \mathrm{~mm}$ graft at $15 \mathrm{ml} / \mathrm{min}$.

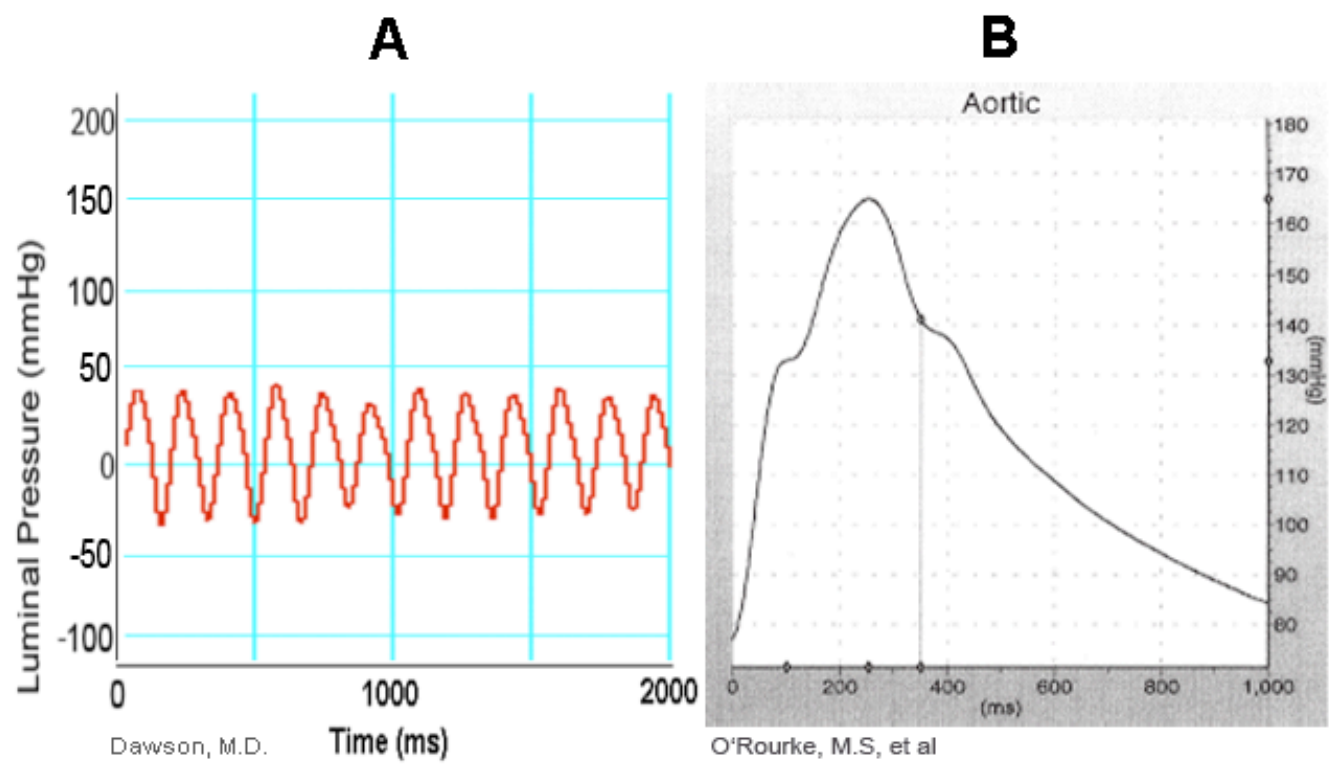

Figure 1.4 (A) Pressure waveforms of steady flow BVM and (B) native arteries [B from 60]. (Permission to reprint figure granted by British journal of clinical pharmacology. See Appendix D) 
A primary principle in tissue engineering involves the creation of an environment that best simulates the physiologic conditions in which the target tissue grows with the idea that this will produce a tissue that is more like the native tissue [11,54]. The engineered tissue must be of the appropriate cell type and structure and in the case of TEVGs, one of the main issues is orientation and development of the medial and intimal layers [50]. Two key factors that affect the cell orientation in vascular grafts, and therefore must be addressed in the in vitro BVM system as well, are flow conditions and shear stress experienced by the cells in the endothelial layer $[10,29,33,50,58,64,85]$. Thus, the flow in a TEVG or the BVM bioreactor chamber would ideally mimic or simulate specific aspects of the conditions found in native vessels; the varied pulsation characteristic of cardiac output as well as the shear stress created by the viscosity of blood.

Importance of Pulsatile Flow

Pressure fluctuations within native blood vessels are a natural occurrence due to the cyclic nature of the heart [61]. This cyclical change in hydrostatic pressure creates varying hoop stresses on the walls of the vessels resulting in varied strain in the tissue of the vessels [69]. As the pressure increases in the vessel the luminal diameter increases slightly. This change in the diameter results in axial strain that, according to Poisson's effect, results in transverse strain in the material [40]. These strains are thought to induce a cellular response that affects cell differentiation during vessel development, mechanical properties, and cell orientation $[69,84,88]$. Many methods have been developed to achieve proper mechanical stimulation and they all have presented similar results with the 
overall finding that in order to produce a native-like vascular graft in vitro, cultivation should be carried out in the presence of pulsatile flow. $[10,68,58,69]$

As described by Seliktar et al [68], when rat Smooth Muscle Cells (SMCs) were cultured and grown in collagen-based gel scaffolds under pulsatile conditions, there was an observed improvement in mechanical strength, SMC orientation, and collagen fibril organization. After 4 and 8 days of growth under pulsatile flow at $1 \mathrm{~Hz}$ to obtain 5 and $10 \%$ strain, the constructs grown under $10 \%$ strain had better collagen lattice reorganization than those grown under $5 \%$ strain. Subsequently, the mechanically conditioned constructs exhibited better mechanical properties and contractile function than the unconditioned controls grown under steady flow. Histologic examination also revealed that the constructs grown under pulsatile conditions showed improved circumferential SMC orientation and collagen fibril orientation when compared to the unconditioned control constructs [68]. Therefore, this study supports the notion that implementing physiologic conditions in tissue-engineered blood vessels is key to producing a native-like construct.

Similar results were found in a 1999 study lead by Niklason et al [58]. This group focused on the development of smaller diameter $(<6 \mathrm{~mm})$ autologous arteries that could be used in bypass grafting. Bovine aortic smooth muscle cells were seeded onto a Polyglycolic Acid (PGA) scaffold where they were grown under pulsatile flow for eight weeks. The gross appearance of the vessels at this time point was stated to be "identical to that of native arteries" and further histology showed migration of the SMCs in a manner that would provide an ideal surface on which ECs could be seeded. In contrast, the control constructs grown under steady flow exhibited no such migration and 
organization. Histologic and mechanical analysis of the constructs revealed little difference in the SMC density, but considerable improvement in suture retention strength and collagen content was seen in the pulsed constructs. With the knowledge that pulsation produces better phenotypic examples of vascular constructs, the group then tested the constructs in animal models. Both the pulsed and non-pulsed grafts were implanted in the right saphenous artery of swine. The non-pulsed grafts developed thrombosis after 3 weeks while the pulsed grafts remained patent after 4 weeks post implant. Although they cited the need for longevity studies of their grafts, results showed that pulsatile conditions are important in the development of a successful TEVG.

Many studies confirm the need for pulsatile flow to produce a vascular graft with native-like properties suitable for implantation. However, the majority of these studies have been performed using extensive culture times with complex bioreactor systems $[58,68]$. One of the objectives of our system is to develop a high throughput model with minimal complexity. Therefore, we will look to develop a method of achieving similar results with less equipment and components.

\section{Importance of Wall Shear Stress}

Although wall stretch via pulsatile flow is important to vessel development, shear stress has also been shown to be of particular importance in the phenotypic development and proper orientation of the vascular endothelial cell layer $[12,25,26,45,86]$. The flow of a fluid through a tube, in this case blood through blood vessels, creates tangential or shear forces on the inner walls of the tube [1,42]. These wall shear stresses are directly proportional to the velocity at which the fluid flows and the viscosity of the fluid $[1,82]$. 
The flow of blood through a vessel creates shear stresses on the endothelial layer that vary constantly depending on location or physiologic needs that can alter heart rate and/or blood viscosity $[26,37]$. There is, however, a constant baseline shear when the heart rate and blood viscosity are at their lowest. Consequently, it has been determined that shear forces have considerable influence on the development and progression of conditions involving the EC layer such as atherosclerosis and restenosis $[46,74,83]$. Similarly, it has been shown that shear stress affects the alignment of SMCs as well [42]. With this in mind, controlling the shear stress seen at the graft wall of our BVM system is very desirable, as it would allow the study of intravascular devices under varying conditions.

Due to its significance in cellular response, there have been many studies on the effect of shear stress on EC and SMC orientation and development $[25,26,42,45,86]$. It has been shown that shear stress affects the phenotypic behavior of the individual vascular cells, and as a result, alters the macroscopic characteristics of blood vessels and their responses to diseases $[37,46,74,83,89]$.

As vessel physiology dictates, endothelial cells experience direct contact with blood flow and thus are directly and immediately affected by shear forces caused by blood flow. Malek et al showed that shear stress induces macroscopic organization of bovine aortic endothelial cells (BAECs) as well as changes in the activity of several intracellular signaling cascades that affect the cellular cytoskeleton [45]. The alignment of the cells is thought to be important in controlling the shear forces experienced by the cells and the changes in the structure of the cells themselves is believed to be important 
for their ability to withstand increased shear forces that may be imposed during increased physical activity [45].

Shear stress has also been found to induce changes in endothelial progenitor cells (EPCs), indicating that its presence is not only important to the maintenance of existing vessels, but to angiogenesis as well [86]. By exposing EPCs to low stress levels (0.1 -2.5 dyne $/ \mathrm{cm}^{2}$ ) for 24 hours, Yamamoto et al observed differentiation, proliferation and subsequent morphologic changes consistent with the development of mature ECs [45]. This occurrence was verified by time-dependent increases in levels of genotypic expression factors and adhesion molecules. Consistent with its role in promoting proliferation and differentiation, shear stress was also found to promote tube formation by EPCs [45]. Capillary-like tubes not only appeared more rapidly, but also the length of the tubes was dramatically longer in cultures grown under increased shear [45]. Although this study regarding the behavior of EPCs is not directly relevant to this thesis, it illustrates that shear stress is important to vascular cells from their inception and lays groundwork for potential future work using our system.

Shear stress, independent of luminal pressure, has been shown to affect the behavior of blood vessels by an observed dilation of arterioles in response to increased flow velocity and/or perfusate viscosity, both of which result in increased wall shear stress [37]. Koller et al isolated arterioles from rat cremaster muscle and cultured them using a perfusion bioreactor system in the absence of dilation signaling agents with constant pressure of $60 \mathrm{mmHg}$ [37]. Experimental runs were done using vessels with and without the endothelial layer [37]. Stepwise increases in shear stress resulted in stepwise increases in the diameter of vessels with the endothelial layer intact, while vessels that 
had the EC layer removed showed almost no dilation [37]. In addition, higher shear levels elicited a greater degree of vasodilation when compared to lower shear [37]. Along with the observed change in cell alignment, this result may be evidence of autoregulation of the wall shear stress by ECs that may serve as an autoprotective mechanism, which further regulates and maintains the state of blood vessels throughout the body.

Along with its effects on the development and maintenance of healthy vessels, shear stress has been implicated in the development and progression of vascular disease $[46,74]$. As a follow up study to their work on the role of shear stress in the changes of endothelial shape cytoskeletal structure, Malek et al looked into the effect that shear stress has on atherosclerosis [46]. Although this disease is related to risk factors including genetic predispositions and lifestyle trends, this study claims that atherosclerotic development is primarily a geometric disease. This claim is based on the fact that physiologic shear stress $\left(\sim 15 \mathrm{dyne} / \mathrm{cm}^{2}\right)$ improves phenotypic EC expression and atheroprotective qualities while low shear stress $\left(<4 \mathrm{dyne} / \mathrm{cm}^{2}\right)$ induces atherogenic cell type [46]. By analyzing the shear stress characteristics of different regions of native blood vessels, a correlation has been shown between areas of decreased shear stress and an increased incidence of atherosclerotic plaques [46]. Areas of this type include natural vessel bifurcations as well as surgically altered vessels where shear stress is changed by the characteristics of flow [46]. As seen in Figure 1.5, reduced shear stress is consistent with the formation of atherosclerotic plaques [46]. Stone et al confirmed the effects of decreased wall shear stress in the thickening of arterial walls and atherosclerotic plaques with little to no change in the luminal diameter and observed an increase in intima-medial 
thickness in stented arteries [74]. Due to the fact that a stent decreases the effective diameter of a vessel, it was found that the region in and around the stented portion of the vessel experienced increased shear stresses and as a result, thickening of the vessel wall due to neointimal hyperplasia that resulted in an increase in a decrease in the luminal diameter [74]. This may contribute to restenosis seen in patients who have received vascular stents.

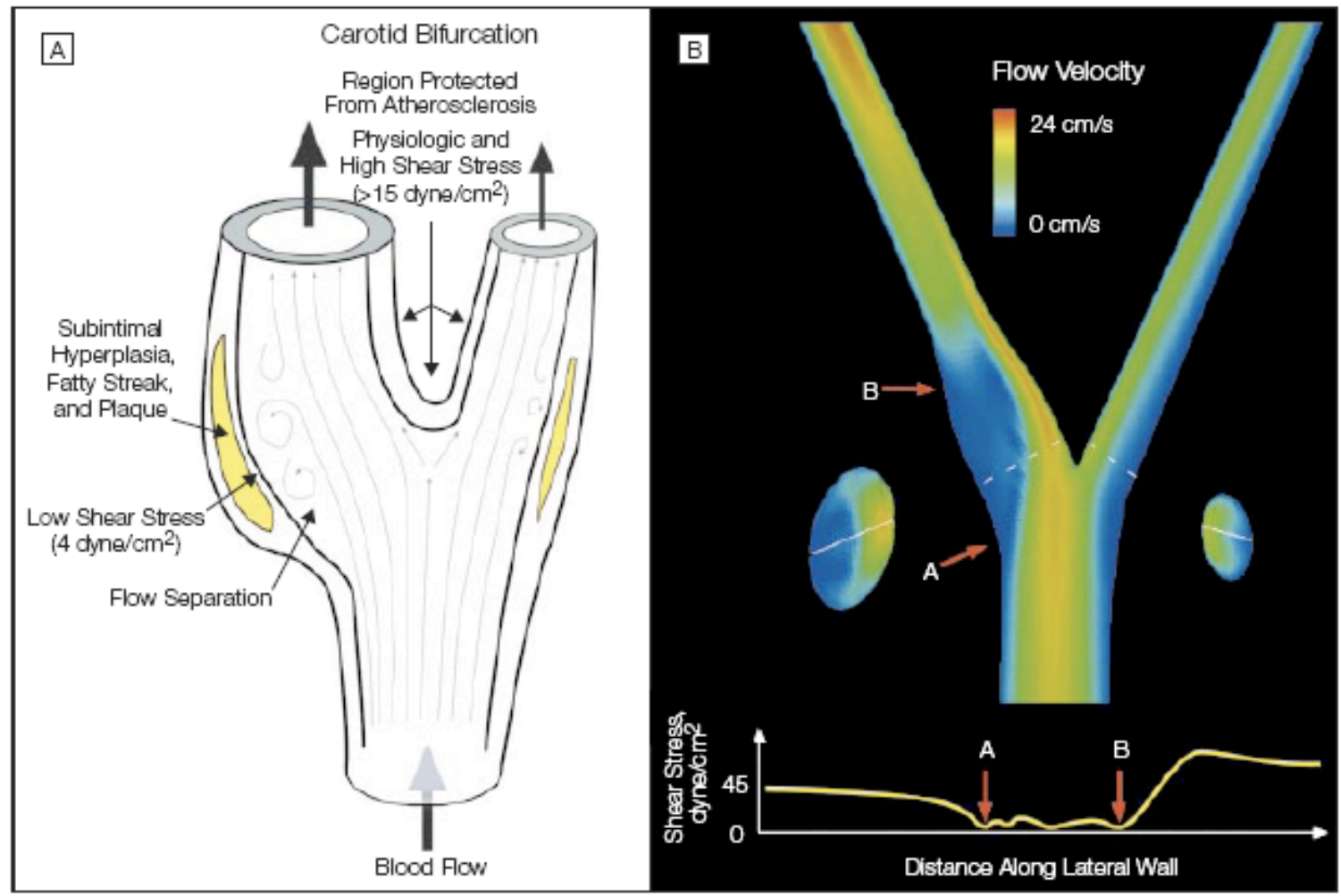

Figure 1.5 Flow analysis of arterial bifurcation. (A) Schematic illustration of the focal nature of atherosclerosis and its tendency to involve the outer walls of vascular bifurcations such as the carotid, coronary, renal, and iliac artery flow dividers. (B) Velocity map of the carotid bifurcation at end-systole using computational fluid dynamic modeling illustrates the lower velocities seen at the outer lateral edges (blue). The computed wall shear stress (bottom) shows the focal low shear magnitude at the outer walls that correspond exactly to the atherosclerosis-prone areas of the carotid bifurcation (compare with $B$ ) and is in contrast with the less susceptible inner regions of the bifurcation where flow velocity and, consequently, hemodynamic shear stress at the vessel wall is higher (yellow and green). (Figure and caption from [46].) (Permission to reprint figure granted by JAMA. See Appendix D) 
Although the role of shear stress in the development of plaques will not be directly explored in this thesis, an understanding of it is relevant to this work as it is further evidence that proper shear stress is of great importance to the proper development of blood vessels and therefore, an important aspect to include in our system. In addition, this information could be used in future work with our system to assess the validity of various stent designs and their effect on plaque development, tendency for restenosis and/or the overall flow characteristics of the vessel.

\section{GOALS OF THIS THESIS}

In continuance of research and development of a Blood Vessel Mimic bioreactor system [12], this thesis aimed to improve the current system by implementing physiologic flow conditions that more accurately represent a native vessel for use in intravascular device testing. This work was carried out in two phases. The first phase was the development and characterization of a pulsatile flow environment similar to that found in the native human circulatory system. The second phase was the formulation and implementation of a growth media to generate an increased shear force at the endothelial cell surface. Upon completion of the development of these features, each was implemented into the BVM system to assess whether or not the engineered vessel would withstand the adulterated conditions.

\section{HYPOTHESES}

The initial focus of this thesis was to establish pulsatile flow. The current system relies on a peristaltic pump to create steady flow through the graft. It is hypothesized that 
a similar peristaltic method can be used to establish pulsatile flow by incorporating a different pump head and adding backpressure to establish appropriate pressure fluctuations. Although simulating the pressure fluctuations exactly as found in vivo would be ideal, the primary objective of this thesis will be to create pressure waveforms similar in shape to physiologic blood pressure while focusing on the pressure differential rather than the ultimate pressure levels. Pulsatile flow in the system will be established and controlled by altering the system configuration while monitoring pressure levels using a pressure transducer to achieve desired waveforms. Initial testing will involve altering the system settings under real-time pressure monitoring to establish and specify settings that can later be used to consistently establish system pressure during graft cultivation.

The second phase of this thesis was to increase and control the shear forces on the endothelial layer. The current system uses a growth media with unspecified viscosity or shear rate. It is hypothesized that the addition of a media supplement can be used to augment and control viscosity and thus the wall shear stress experienced by the cells grown in the construct. The viscosity of the augmented media will be determined and specified for varying levels of the thickening agent using a Vilastic-3 viscometer This study will test two different additives that will be used to control the viscosity of the growth media and thus the shear force experienced by the cells. The two agents that will be tested are Methyl Cellulose and Dextran, both of which will be used in varied concentrations to create varied viscosities. It is important that the supplement not negatively impact cell growth and both of these reagents have been used in cell culture and are thought to be capable of establishing the desired viscosity while not impeding the 
growth characteristics of the cells involved [24,25], an aspect that will be tested prior to its introduction to the system. The supplement will be used in a concentration that establishes the desired shear stress while maintaining some degree of cell adhesion on the scaffold. Again, this is a minor alteration of the original system that is thought to be a practical solution to the need for the presence of controllable shear force where there is currently none. 


\section{Chapter 2: DEVELOPMENT OF PULSATILE FLOW SYSTEM}

\section{INTRODUCTION}

As the cardiac cycle and the nature of the heart as a pump dictates, the flow in the cardiovascular system exhibits pulsatile flow $[36,39,60]$. Noninvasive techniques have allowed the collection of data used to generate pressure waveforms demonstrating this pulsatile quality $[36,60]$. Pulsatile hemodynamic forces result in transmural strain that the vessel must resist with circumferential hoop stress that can be estimated with Laplace's Law; $\sigma=P \bullet R / t(\mathrm{P}-$ luminal pressure, $\mathrm{R}$ - vessel radius, $\mathrm{t}$ - vessel wall thickness [39].

In native blood vessels, this cyclic fluctuation in hoop stress has physiologic effects that induce arterial remodeling and alteration of vessel properties [41] by eliciting responses from smooth muscle cells (SMCs) and endothelial cells (ECs) [28,41,62,69]. Mechanical stretching of the vessel wall has been shown to affect the phenotype, orientation, extracellular matrix (ECM) deposition and growth factor release in SMC cultures [28]. These changes in the SMC layer are significant as they improve the mechanical integrity of the vessel and the presence of growth factors may improve phenotypic expression and orientation of not only the SMCs, but the EC layer as well [28].

The effects of pulsation have also been shown to directly affect the phenotypic expression of ECs $[21,23,62]$. This has been revealed through the detection of the release of an Endothelium Derived Hyperpolarizing Factor (EDHF) in porcine coronary arteries [62]. EDHF is a contributor to vessel size and is a component in the regulation of vessel 
constriction and relaxation [79]. Not only has pulsatile stretching been shown to simply affect the release of EDHF but it has also been found that it actually enhances its expression and activity in coronary arteries [21]. Along with enhancing EDHF expression, it has been demonstrated that pulsation affects the basic EC phenotype as well with an observed population of "normal" cells in regions of the vessel where strain resulting from luminal pressure was the greatest [23].

Due to the demonstrated importance of pulsation in creating a phenotypically accurate vascular graft, the development of a TEVG for implantation or device testing must include a method for simulating native pulsatile flow produced by the heart. Many approaches have been taken in attempting to best achieve this goal by groups aiming to produce a viable, implantable TEVG. There are several examples of nearly ideal waveforms, however the systems developed by these groups are rather complex and include many components that must be separately tuned and maintained $[28,29,60,85]$.

To simulate physiologic pulsatile flow, M.S. Hahn et al employed modified Cellmax pumps from Spectrum Labs to control pressure and thus pulsation within their system. A peristaltic pump drove primary flow of the perfusion fluid, which then passed through a compliance chamber to damp pulsation created by the peristaltic pump. Fluid then passed through the Cellmax pumps, which controlled pulsation by compressing or massaging the tubing. Check valves were placed before and after each Cellmax pump to prevent back flow. A multi-construct chamber was placed downstream of the Cellmax pumps with an inline pressure transducer to monitor fluid pressure at the construct chamber while the system was running [28]. A diagram of this system and resulting pressure waveforms can be seen in Figure 2.1. One key aspect of this system is that 
pulsation is controlled externally to the flow path with the intent of reducing the opportunity for contamination by avoiding media contact with pump components.
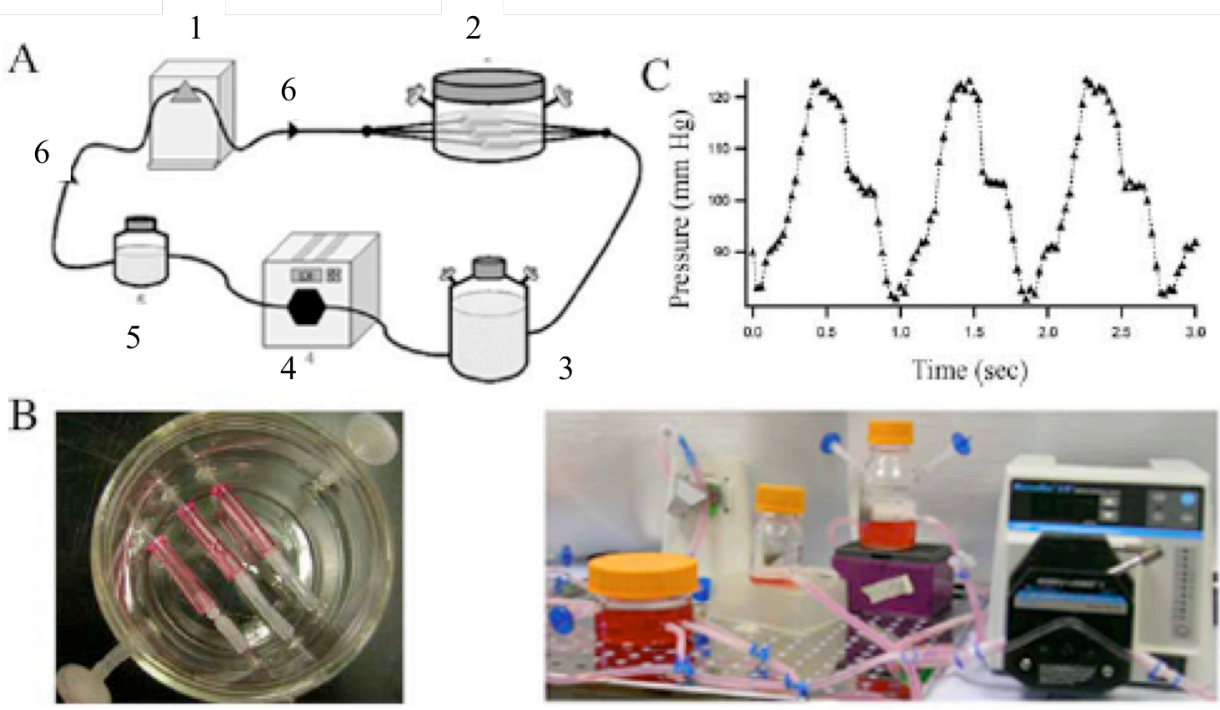

Figure 2.1 Pulsatile flow bioreactor by Hahn et al (A) Schematic of system design, which includes (1) one or more pulsatile pumps, (2) custom graft chamber, (3) media reservoir, (4) a peristaltic pump, (5) compliance chamber, (6) check valves. (B) Close up of the graft chamber. (C) Representative pressure waveforms generated using the bioreactor (D) image of the actual bioreactor system. [28] (Permission to reprint figure granted by Springerlink publications. See Appendix D)

S.P. Hoerstrup et al used a pulse-duplicating pump to create pulsation in their TEVG bioreactor system. Media was drawn into the pulse duplicator from a media reservoir and sent to four vessel constructs housed in a bioreactor chamber and returned to the media reservoir. Pulsation was created by cyclic expansion and relaxation of an elastic membrane within the pulse duplicator. The expansion/relaxation was controlled by an external dual-phase air pump, which acted by inflating and deflating the membrane.

See Figure 2.2 for the layout of this system [29]. 


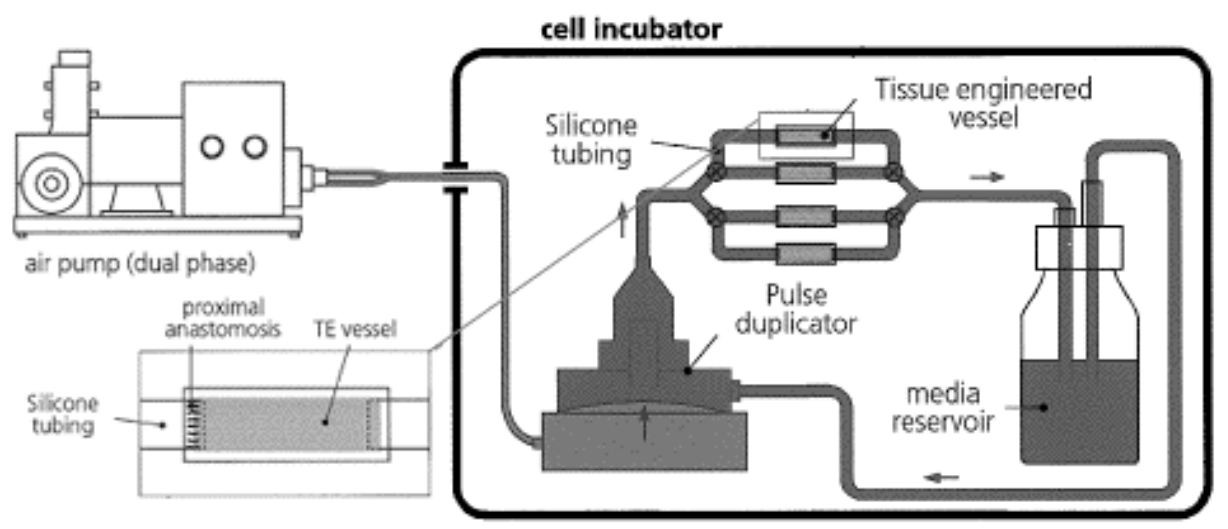

Figure 2.2 Diagram of bioreactor system by Hoerstrup et al [29]. (Permission to reprint figure granted by Elsevier publications. See Appendix D)

Although this system has fewer components than the Hahn system, the growth media passes through and directly contacts the internal workings of the pulse-duplicator, which can result in contamination of the system.

A more simplified approach was taken by Williams and Wick to develop a pulsatile bioreactor system. In this design, cell seeding and cultivation occurred using two different pump configurations in the same basic system. During cell seeding and for 24 hours thereafter a reciprocating dual syringe pump drove luminal flow. Media was concurrently pumped through the growth chamber external to the graft by a peristaltic pump to promote nutrient transport from the outer surface of the construct. Following the 24-hour seeding period, the dual syringe pump was replaced with a peristaltic pump to produce pulsatile luminal flow. This system requires more than one pump configuration, however pulsation is derived solely from a single peristaltic pump without the need for compliance chambers or flow controllers. The means for achieving pulsatility is this system are similar to our system, however there are two separate flow paths and flow meters in place that make it more complex than ours. 
Due to the demonstrated need for pulsation in an in vitro TEVG or BVM bioreactor system, the goal of the work presented in this thesis was to make changes to the current BVM system, described in Chapter 1, that would allow for an accurate simulation of the pressure fluctuations experienced by native vessels. In describing this work, the development and implementation of the alterations to the system will be discussed along with the results of preliminary BVM construct cultivation under pulsatile flow.

\section{MATERIALS AND METHODS}

The development of pulsatile flow in our system was approached with the objective of making moderate modifications meant to minimize changes to the overall usability and simplicity of the current setup. Thus, prior experience with peristaltic pump systems directed the thought that the desired pressure fluctuations could be attained by selecting a different pump head, specifying a larger diameter tubing for use at the roller interface, and slightly modifying bioreactor system configurations.

\section{Pump Head and Tubing Selection}

For the desired pulse and flow rates, a 3 and 4-roller pump head (Cole Parmer P/N HV- 07519-05 and HV-07519-06) and pump tubing from Masterflex were considered. Data regarding the flow rates expected from different pump head and tubing combinations at varied RPMs was obtained from the manufacturer. In order to make the best initial choice of pump head configuration and tubing size for our application, these data were used to extrapolate estimations of flow rates at pump speeds that would be used 
in this system. The actual flow rates were later tested to determine true flow rates at specified pump settings.

There were several material types available in the L/S 16 (Cole Parmer Model number) size and after studying their qualities; the Platinum Cured Silicone material (Cole Parmer P/N 96440-16) was selected for its biocompatibility and permeability to gases. The gas permeability of the tubing is important as it allows for oxygen and carbon dioxide exchange in the growth media without the need for a sterile vent filter on the media reservoir.

\section{Actual Flow Rate vs. Pump Speed}

The original design of our BVM bioreactor system calls for flow rates between 10 and $15 \mathrm{ml} / \mathrm{min}$ [12]. Since our lab uses different pump drives and pump heads than were used previously, it was necessary to determine what RPM settings on our pumps would produce the desired flow rates. This was determined by simply measuring the displaced volume after one minute at various RPMs. This was performed for both the steady flow setup with the 8-roller pump head and the pulsatile setup with the 3-roller pump head.

\section{Pressure Monitoring System}

Although it was hypothesized that pulsatile flow could be established using a new tubing and pump head setup, the degree to which the pressure fluctuation could be introduced and controlled was unknown. To quantify this characteristic of the system, an AD Instruments Powerlab 4/25 data acquisition system was used. System pressure was 
detected using a blood pressure specific, reusable pressure transducer (ADI $\mathrm{P} / \mathrm{N}$ MLT0699) and recorded using the LabChart software.

Prior to taking pressure readings for the BVM setups, the ADI recording system was calibrated for a $0-200 \mathrm{mmHg}$ range using a mercury-filled manometer to establish a two-point calibration that allowed the software to translate the incoming signal into accurate pressure readings. Along with its particularly ideal applicability to this study in its ability to accurately measure pressure changes in the physiologic range with great sensitivity, the ADI system's software also has the advantage of being able to further extrapolate data using preset or user defined algorithms. Therefore, not only were instantaneous pressure readings and changes recorded, but also corresponding pulse and flow rates could be calculated and plotted at different pump speeds. Table 2.1 outlines the overall approach and tests that were performed during the characterization work.

Table 2.1 Outline of tests performed using AD Instruments Data Acquisition System.

\begin{tabular}{|c|c|c|c|c|}
\hline System Configuration & $\begin{array}{c}\text { Pump } \\
\text { Head }\end{array}$ & $\begin{array}{c}\text { Perfusion } \\
\text { Fluid }\end{array}$ & $\begin{array}{c}\text { Pump } \\
\text { Tubing/ID }\end{array}$ & Measurement/Goal \\
\hline Original Steady Flow & 8-roller & water/media & L/S 14/1.6mm & Pressure Profile \\
\hline Original Steady Flow & 8-roller & water & L/S $14 / 1.6 \mathrm{~mm}$ & Flow Rate \\
\hline Basic Loop & 3-roller & water & L/S $16 / 3.1 \mathrm{~mm}$ & Pressure Profile \\
\hline Basic Loop with Reservoir & 3-roller & water & L/S $16 / 3.1 \mathrm{~mm}$ & Pressure Profile \\
\hline Pulsatile -Original Chamber & 3-roller & water/media & L/S 16/3.1mm & Flow Rate \\
\hline Pulsatile -Original Chamber & 3-roller & water/media & L/S $16 / 3.1 \mathrm{~mm}$ & Pressure Profile \\
\hline Pulsatile -Original Chamber & 3-roller & water/media & L/S $16 / 3.1 \mathrm{~mm}$ & Establish BP Models \\
\hline Pulsatile -Updated Chamber & 3-roller & water/media & L/S $16 / 3.1 \mathrm{~mm}$ & Flow Rate \\
\hline Pulsatile -Updated Chamber & 3-roller & water/media & L/S $16 / 3.1 \mathrm{~mm}$ & Pressure Profile \\
\hline Pulsatile -Updated Chamber & 3-roller & water/media & L/S $16 / 3.1 \mathrm{~mm}$ & Establish BP Models \\
\hline
\end{tabular}

\section{Characterization of Original Steady Flow BVM System}

With the pressure recording system calibrated, the flow characteristics of the original steady flow system were examined. A system was set up exactly as originally designed [12] with the exception of the incorporation of the ADI MLT0699 pressure 
transducer which was placed inline via luer lock connections just downstream of the BVM chamber (Figure 2.3). It was assumed that the addition of the pressure transducer to the system would not alter the flow or pressure fluctuations, as the inner diameter of the transducer was the same as that of the tubing used throughout that of the BVM system. Water was used in place of growth media for its cleanliness and to avoid the cost of wasting expensive media. Once the system was understood and consistent results could be achieved, readings were taken using growth media to ensure that it would produce similar results. Figure 2.4 shows the entire system as it was configured for steady flow pressure characterization.
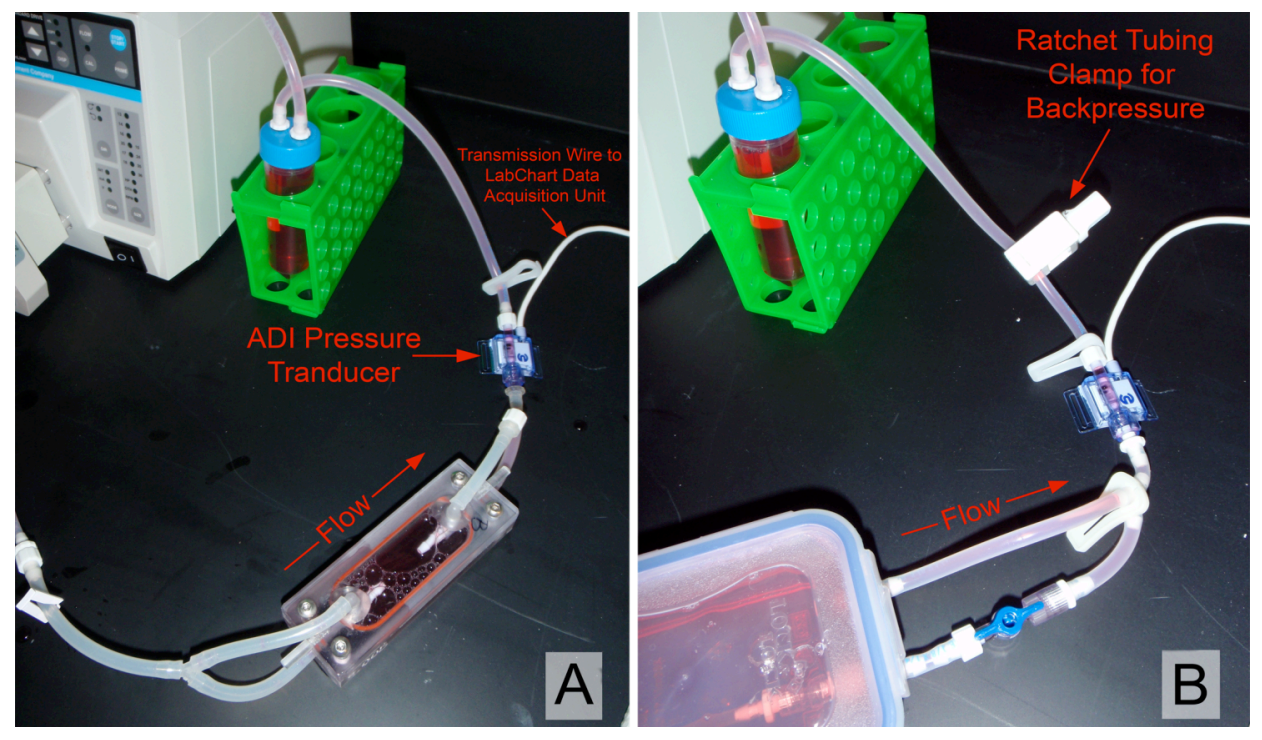

Figure 2.3 Placement of AD Instruments pressure transducer in steady flow (A) and pulsatile (B) configurations. 


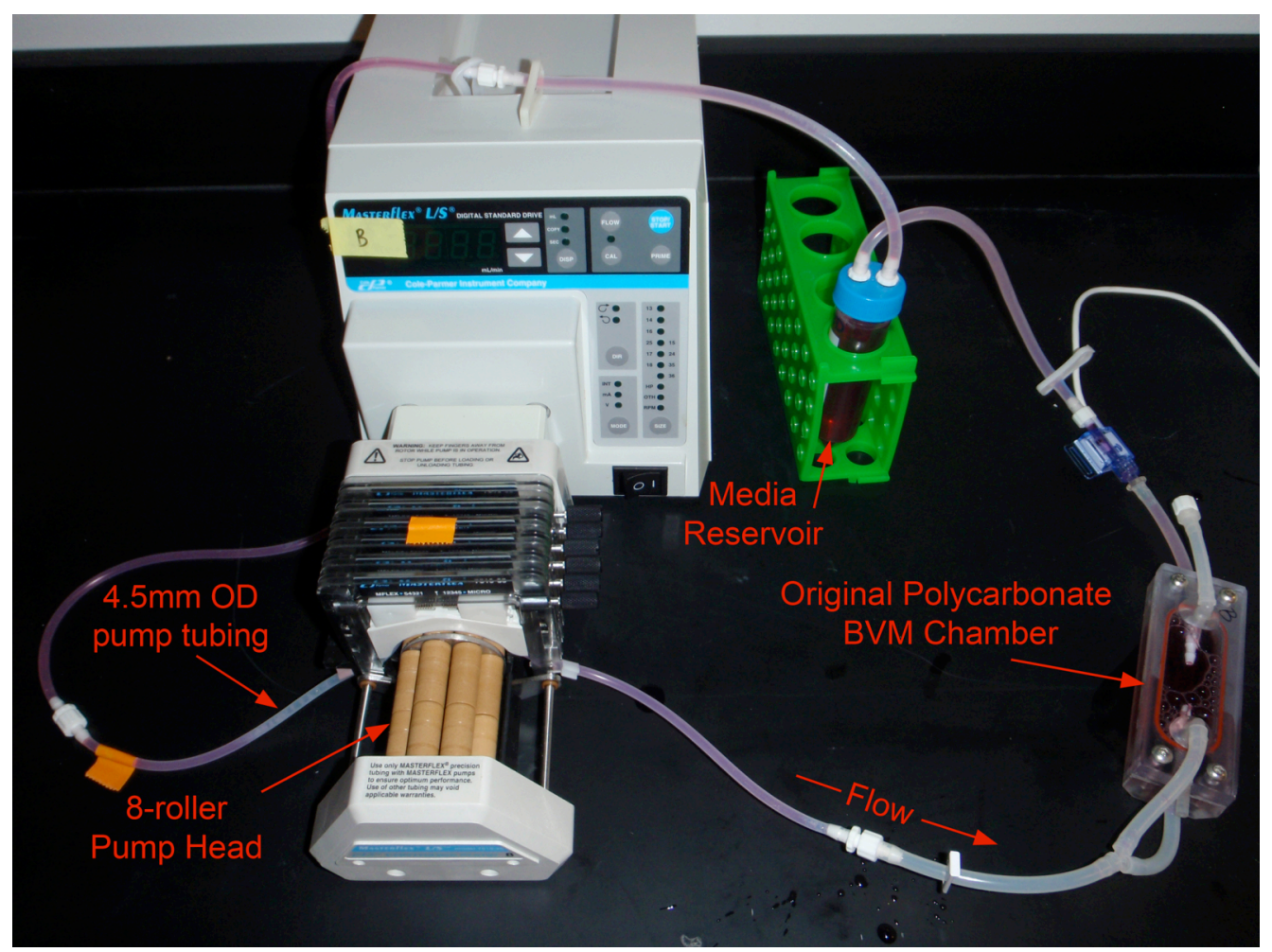

Figure 2.4 Steady flow configuration set up for pressure characterization.

\section{Characterization and Development of the Pulsatile Flow Setup}

With the pressure profile of the original steady flow setup established, the development and characterization of the pulsatile setup commenced. Initial testing involved a simple closed loop of Masterflex L/S 16 tubing driven by the 3-roller pump head with the same ADI pressure transducer in place. This testing was carried out using water at an RPM of 20 to simulate a heart rate of 60 BPM.

In order to pressurize the system and create pulsation, a stopcock was put in place downstream of the pressure transducer to regulate backpressure. Although an increase in pressure was seen, there was a subsequent vacuum following each positive spike. It was deduced that this negative pressure spike would be detrimental to the growth of the BVM as it could possibly pull the cells off of the scaffold or hinder proper seeding and 
development. Thus, a reservoir was added to the simple loop setup just upstream of the pump head and downstream of the backpressure stopcock.

The follow-up to the initial studies with the simple, closed loop involved the incorporation of the BVM chamber. The chamber was placed in the system just upstream of the pressure transducer and downstream of the pump head. The stopcock remained downstream of the pressure transducer to control backpressure in the BVM chamber. At this point, the system was configured exactly as it would be when used to culture the BVM including the ePTFE scaffold in the chamber, with the exception of the perfusion fluid, which at this point was water (Figure 2.4).

It was quickly determined that the standard stopcock did not allow for fine adjustments in back pressure as there was a very small margin between regulating restriction and complete stoppage of flow. Therefore, a roller clamp was implemented as a possible solution. Instead of acting as an inline flow controller, the roller clamp mounts external to tubing and acts by "pinching" the tubing between a roller barrel and an angled ramp resulting in a gradual increase in flow restriction. Not only was it thought that this clamp type could better control flow, but it also mounted external to the sterile barrier of the tubing, thus reducing the opportunity for contaminates to become lodged in the crevices of a stopcock.

As a potential improvement over the roller clamp, a ratchet screw tubing compressor from Cole Parmer (P/N WU-06833-10 -Figure 2.3) was tested on the ADI Powerlab system following the same procedure used for the other two clamps. Each target pressure was indexed on the clamp to allow the settings to be established and set in the system without the use of the pressure sensor. This is desirable, as the system needs 
to be pressurized in the $\mathrm{CO}_{2}$ incubator where the pressure monitoring system is not available (See Appendix A). One additional feature of the ratchet clamp is that it can be attached to up to three tubes at once allowing for identical pressure settings in up to three systems at once. Figure 2.5 illustrates the system as it was set up for pulsatile flow.

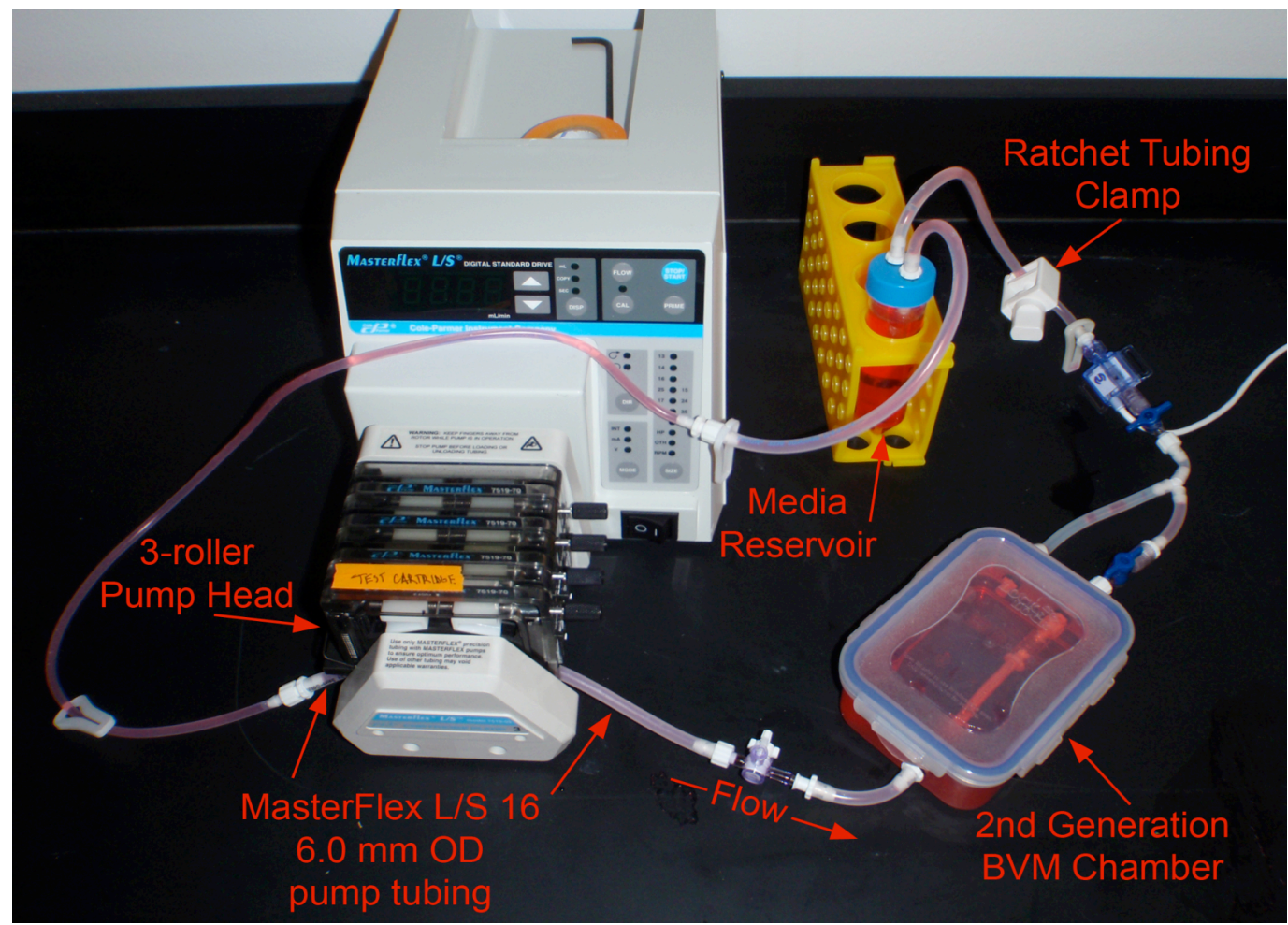

Figure 2.5 Pulsatile flow configuration set up for pressure characterization.

\section{Development of Blood Pressure Model Settings}

Following the establishment of desired pressure waveforms in the complete BVM system, the next step was to attempt to specify settings for several different models that would simulate conditions of the cardiovascular system. One of the benefits of this in vitro system lies in its tunability. Pulse rate and pressure fluctuation can be adjusted independently which would allow for modeling of conditions such as hypertension or tachycardia. 
To show that the system can simulate varied physiologic conditions, the flow setup was adjusted while monitoring with the ADI Powerlab system to determine the settings for three example blood pressure models. Settings were established for: normal adult, mild hypertensive and moderate hypertensive models. Table 2.2 indicates target pressure ranges for the each of these models.

Table 2.2 Target pressures for BVM Blood Pressure Models [80].

\begin{tabular}{|c|c|c|}
\hline $\begin{array}{c}\text { Cardiac Blood } \\
\text { Pressure } \\
\text { Condition }\end{array}$ & $\begin{array}{c}\text { Systolic Cardiac } \\
\text { Pressure }(\mathrm{mmHg})\end{array}$ & $\begin{array}{c}\text { Diastolic Cardiac } \\
\text { Pressure }(\mathrm{mmHg})\end{array}$ \\
\hline Normal & 90 & 60 \\
\hline Normal & 100 & 65 \\
\hline Normal & 110 & 70 \\
\hline Normal & 120 & 80 \\
\hline Normal & 130 & 85 \\
\hline Normal/High & 140 & 90 \\
\hline Mild High & 150 & 95 \\
\hline Moderate High & 160 & 100 \\
\hline Moderate High & 170 & 105 \\
\hline
\end{tabular}

With the pressure waveforms of the fully equipped system validated, pressure readings were taken using growth media to establish that the use of media in the system would not alter the pressure characteristics found when testing with water.

\section{Preliminary Cell Adhesion Test}

With the ratchet clamp indexed to produce the desired pressure fluctuations for the "normal adult " pressure range, two BVM setups were prepared to determine if the system could maintain long term pressure following the implementation of pulsation. For this initial test, Human Umbilical Artery Smooth Muscle Cells were sodded onto an ePTFE scaffold per the Sodding Protocol (Appendix B). These setups were allowed to grow under steady flow for several days to allow establishment of the culture and initial 
adhesion to scaffold. After 64 hours of steady flow at $\sim 12 \mathrm{ml} / \mathrm{min}$, the system was removed from the 8-roller pump and installed on the 3-roller pump head. The pump tubing was moved from a section of small tubing (OD: 4.5mm) to a section of Masterflex L/S 16 tubing (OD: 6.0mm) with the larger cartridges (Cole Parmer P/N HV-07519-75) used on the 3-roller head. Flow was initiated at $20 \mathrm{RPM}$ to give a flow of $\sim 18.5 \mathrm{ml} / \mathrm{min}$ and a pulse rate of $60 \mathrm{BPM}$. The ratchet clamp was mounted downstream of the BVM chamber on both setups and tightened down on the tubing to initiate pressurization of the systems to $\sim 70 \mathrm{mmHg}$ and pulsation within the graft.

Upon reaching full pressurization of the system, it was noticed that the restriction in luminal flow intended to create backpressure was causing excessive transmural flow. In order to continue the test run, backpressure was reduced to $\sim 30 \mathrm{mmHg}$ and the pump speed was reduced to 15 RPM to restore luminal flow. One of the BVM setups was taken down after 2 hours of pulsation and the second setup was taken down after 24 hours. Once the system was down, the graft was removed and placed in Histochoice to preserve it for later analysis. Due to the problems experienced in these trials, analysis of these particular constructs was not performed in this thesis, however the samples were stored for potential subsequent studies.

Pressure Testing with First and Second Generation BVM Chambers

Due to observed problems with excessive transmural flow during initial cell adhesion tests, the flow characteristics were reexamined on the ADI system with greater scrutiny. The original tests were done without considering the rigidity of the BVM chamber. In order to determine if this would affect the excessive transmural flow seen in 
the initial tests, an older version of the BVM chamber made of rigid polycarbonate with a highly secure lid was tested on the ADI system to determine if it would behave similarly to the updated chamber.

Another aspect of the system that was not considered in initial pressure tests was the state of the ePTFE graft. In initial tests, excessive transmural flow may have been overlooked, as the graft was not denucleated and thus may have been less permeable to the perfusion fluid. To determine whether or not this was a factor in maintaining luminal flow and pressure, pressure tests were done with an ePTFE graft that was denucleated (See Appendix B for the cell sodding protocol).

\section{$\underline{\text { Final Cell Adhesion Tests }}$}

With the pressurization of the system better understood, more extensive cell adhesion tests were performed. Six grafts were sodded with human umbilical vein endothelial cells (HUVECs) and allowed to grow for 72 hours under steady flow. After 72 hours, five of the six setups were moved to the 3-roller pump head and for comparative purposes, these variable groups were maintained at three different pressure settings. The remaining graft was maintained under steady flow on the 8-roller pump head at $15 \mathrm{ml} / \mathrm{min}$ for use as a control. See Table 2.3 for an overview of the test conditions. To avoid disturbing or dislodging the cells from the scaffold, the pump speed was reduced to 10 RPM for the initial application of backpressure and pressurization of the system. The ratchet clamp was tightened to a previously indexed setting and the pressure was then ramped up incrementally by increasing the pump speed by three RPM every hour until the final setting of 20 RPM was established. Once the final pressure and 
pump speed were reached, the constructs were maintained at these settings for 24 hours, at which point all six setups were taken down.

Table 2.3 Pulsatile flow cell adhesion test condition summary.

\begin{tabular}{|c|c|c|c|c|c|}
\hline Group/Construct \# & $\begin{array}{c}\text { Pump Head } \\
\text { Used }\end{array}$ & $\begin{array}{c}\text { Final } \\
\text { Pulsatile } \\
\text { Pressure } \\
(\mathrm{mmHg})\end{array}$ & $\begin{array}{c}\text { Final Flow } \\
\text { Rate } \\
(\mathrm{ml} / \mathrm{min})\end{array}$ & Pump RPM & $\begin{array}{c}\text { Time at Final } \\
\text { Pulse } \\
\text { Pressure } \\
\text { (hours) }\end{array}$ \\
\hline PA1 & 8-roller & unspecified & 15 & 140 & 24 \\
\hline PB1 & 3-roller & unspecified & 18.5 & 20 & 24 \\
\hline PB2 & 3-roller & $\sim 40$ & 18.5 & 20 & 24 \\
\hline PB3 & 3-roller & $\sim 40$ & 18.5 & 20 & 24 \\
\hline PB4 & 3-roller & $\sim 80$ & 18.5 & 20 & 24 \\
\hline PB5 & 3-roller & $\sim 80$ & 18.5 & 20 & 24 \\
\hline
\end{tabular}

To take down the systems, the peristaltic pumps were turned off, stopcocks proximal and distal to the graft chamber were closed and the tubing cartridge and tubing were removed from the pump head. If additional systems were to be maintained, the pump was restarted at the specified RPM. With the setup removed from the pump system, the chamber was opened and the scaffold was carefully cut at both ends using a No. 11 scalpel blade while still submerged in media. Without compressing or bending the scaffold, it was then placed in a vial of Histochoice for preservation until later analysis with SEM and bisbenzimide (BBI) staining (see Figure 2.6). When placing the excised graft in the fixative, the orientation of the graft was marked as to keep track of the distal and proximal ends for analysis purposes. The media was drained from the chamber and the tubing and all parts were washed for reuse.

When all grafts had been in Histochoice for at least 24 hours at room temperature, a portion of the distal end of the fixed graft was cut off. This section was then cut longitudinally in order to separate the upper and lower hemispheres of the graft. These sections were then placed in 1:1000 dilution of BBI stain. After 24 hours in this solution, the samples were analyzed under a fluorescent microscope. 


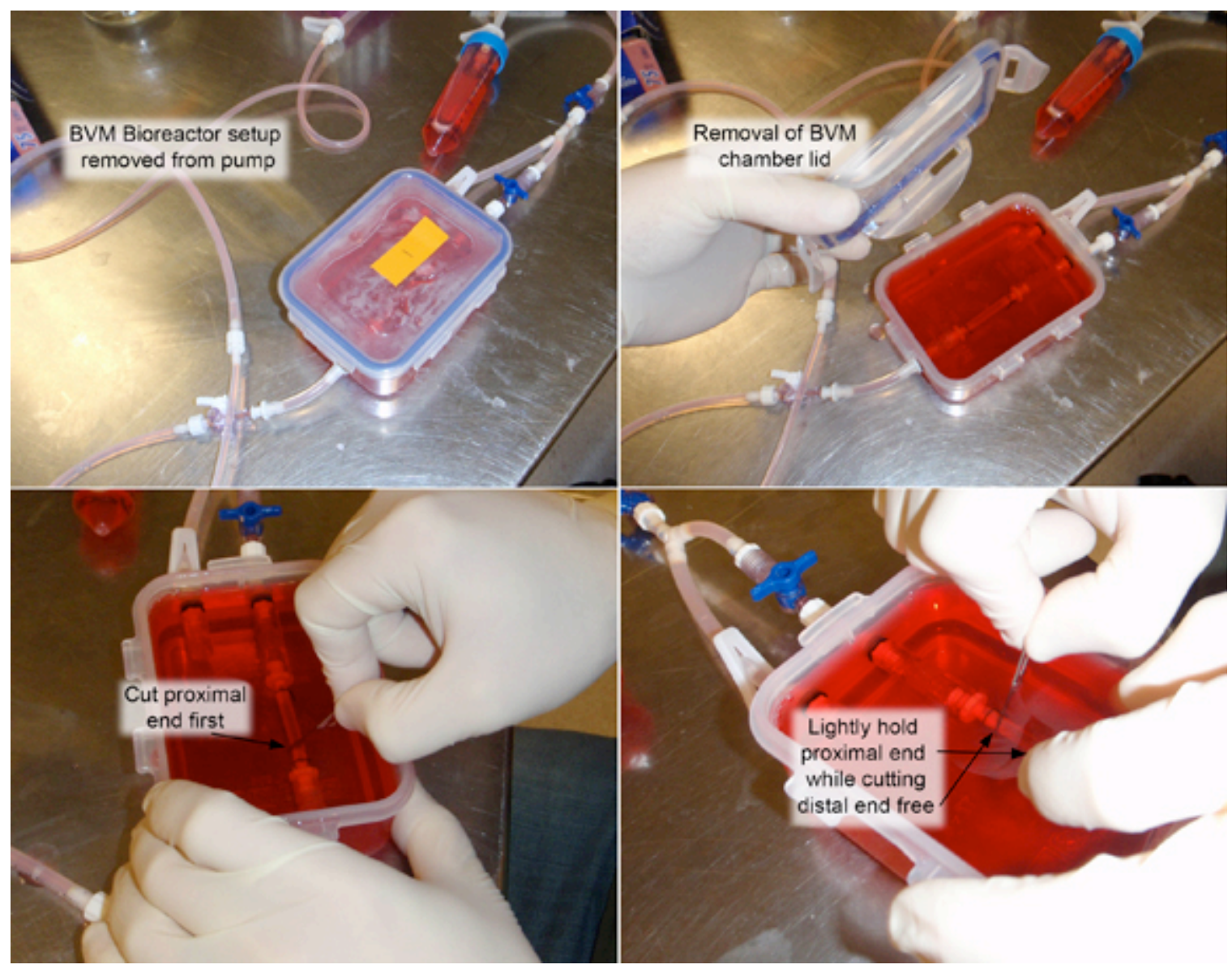

Figure 2.6 Removal of ePTFE graft from BVM bioreactor chamber upon completion of growth period.

After BBI imaging was completed, the sections were placed in a dessicator overnight to dry them out in preparation for Scanning Electron Microscopy (SEM). Once dry, the samples were gold sputter coated for 70 seconds and analyzed in the SEM.

\section{$\underline{\text { RESULTS }}$}

Estimating Flow Rates

The following flow rates were obtained from Cole Parmer (Table 2.4). These data were plotted and the resulting linear equations (Figure 2.7) were used to estimate the flow rates at different pump speeds (Table 2.5 and Figure 2.8). 
Table 2.4 Data for initial comparison of potential pump configurations.

\begin{tabular}{|c|c|c|c|c|c|c|}
\hline \multicolumn{7}{|c|}{$\begin{array}{c}\text { Flow Rate Data Provided by } \\
\text { Pump Manufacturer }\end{array}$} \\
\hline \multirow{2}{*}{$\begin{array}{c}\text { Pump } \\
\text { RPM }\end{array}$} & \multicolumn{3}{|c|}{ FLOW (mL/min) } & \multicolumn{2}{c|}{ Beats/min. } \\
\cline { 2 - 7 } & L/S 13 & L/S 14 & L/S 16 & L/S 25 & 3 roller & 4 roller \\
\hline 0 & 0 & 0 & 0 & 0 & 0 & 0 \\
\hline 6 & 0.3 & 1.3 & 4.6 & 10 & 18 & 24 \\
\hline 9 & 0.45 & 1.95 & 6.9 & 15 & 27 & 36 \\
\hline 12 & 0.6 & 2.6 & 9.2 & 20 & 36 & 48 \\
\hline 18 & 0.9 & 3.9 & 13.8 & 30 & 54 & 72 \\
\hline 24 & 1.2 & 5.2 & 18.4 & 40 & 72 & 96 \\
\hline
\end{tabular}

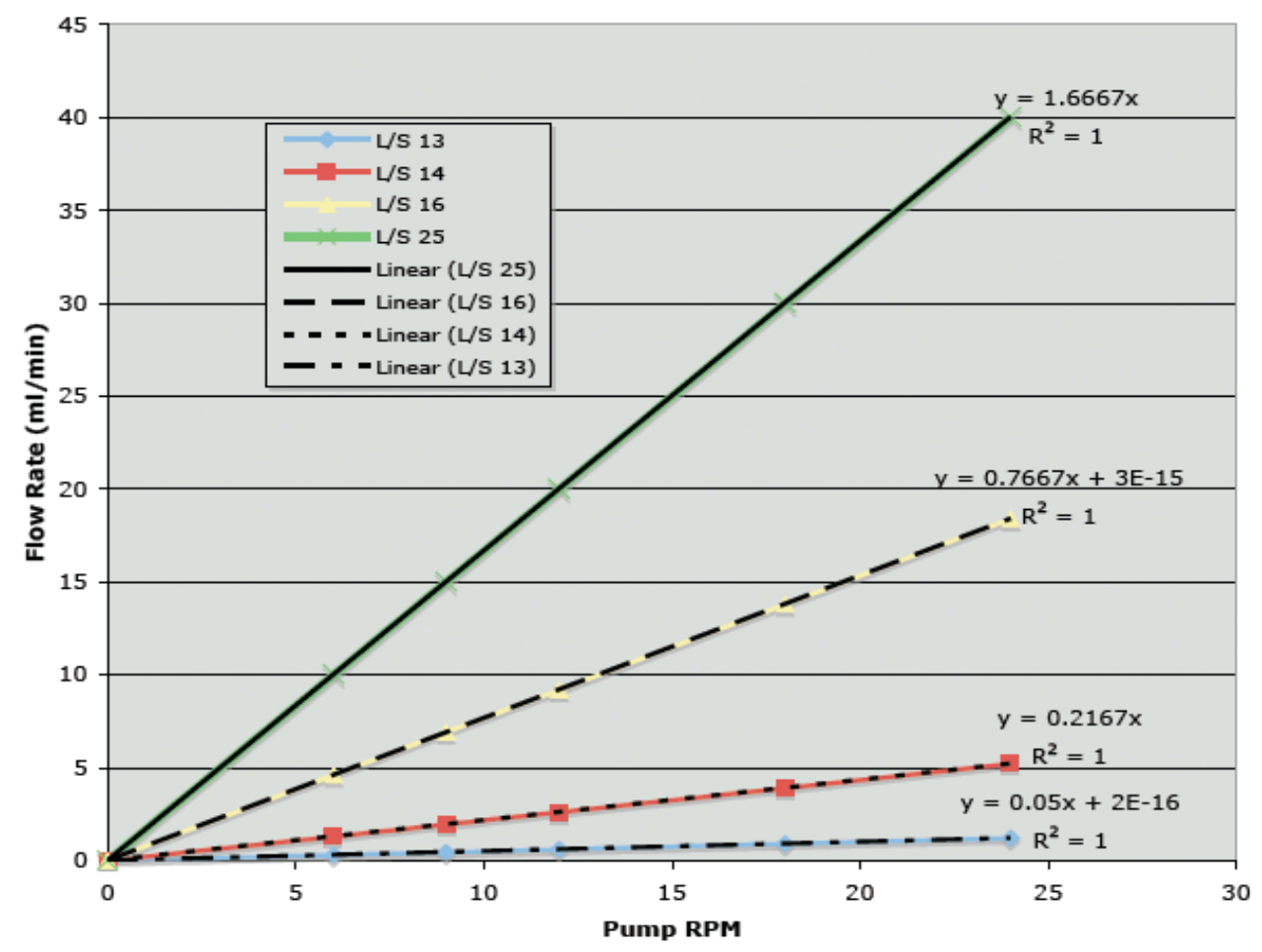

Figure 2.7 Plot of manufacturers estimated flow rates with linear approximations. 
Table 2.5 Flow rates estimated using linear equations extrapolated from manufacturer's data. Blue highlighted flow rates correspond to those specified in the configuration in the original system.

\begin{tabular}{|c|c|c|c|c|c|c|}
\hline \multicolumn{7}{|c|}{$\begin{array}{c}\text { Estimated Flow Rates Extrapolated from } \\
\text { Manufacturer Data }\end{array}$} \\
\hline \multicolumn{7}{|c|}{ Linear Equations Resulting from Plot of Data Above } \\
\hline & L/S 13 & \multicolumn{4}{|c|}{ Flow Rate $=0.05($ RPM $)+2 \mathrm{E}-16$} & \\
\hline & L/S 14 & \multicolumn{4}{|c|}{ Flow Rate $=0.2167($ RPM $)$} & \\
\hline & L/S 16 & \multicolumn{4}{|c|}{ Flow Rate $=0.7667(\mathrm{RPM})+3 \mathrm{E}-15$} & \\
\hline & L/S 25 & \multicolumn{4}{|c|}{ Flow Rate $=1.6667($ RPM $)$} & \\
\hline Pump & \multicolumn{4}{|c|}{ FLOW $(\mathrm{mL} / \mathrm{min})$} & \multicolumn{2}{|c|}{ Beats/min. } \\
\hline RPM & $\mathrm{L} / \mathrm{S} 13$ & $\mathrm{~L} / \mathrm{S} 14$ & $\mathrm{~L} / \mathrm{S} 16$ & $\mathrm{~L} / \mathrm{S} 25$ & 3 roller & 4 roller \\
\hline 15 & 0.8 & 3.3 & 11.5 & 25.0 & 45 & 60 \\
\hline 16 & 0.8 & 3.5 & 12.3 & 26.7 & 48 & 64 \\
\hline 17 & 0.9 & 3.7 & 13.0 & 28.3 & 51 & 68 \\
\hline 19.56 & 1.0 & 4.2 & 15.0 & 32.6 & 58.68 & 78.24 \\
\hline 20 & 1.0 & 4.3 & 15.3 & 33.3 & 60 & 80 \\
\hline 21 & 1.1 & 4.6 & 16.1 & 35.0 & 63 & 84 \\
\hline 30 & 1.5 & 6.501 & 23 & 50 & 90 & 120 \\
\hline 35 & 1.8 & 7.6 & 26.8 & 58.3 & 105 & 140 \\
\hline 40 & 2.0 & 8.7 & 30.7 & 66.7 & 120 & 160 \\
\hline
\end{tabular}

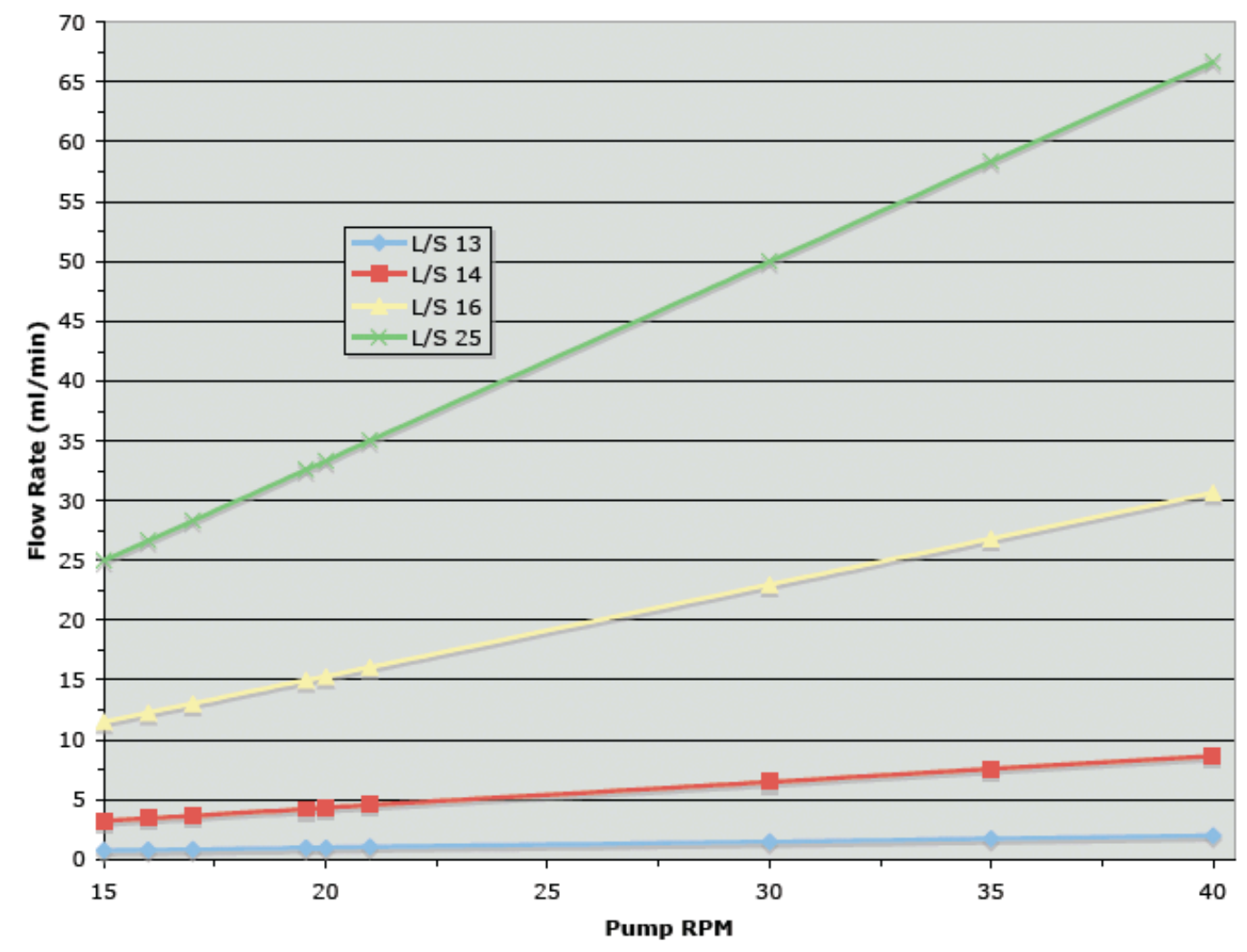

Figure 2.8 Plot of estimated flow rates using different pump tubing diameters.

Based on the estimated flow rates seen in Table 2.5 the Masterflex L/S 16 sized tubing showed the most desirable characteristics when paired with the 3-roller pump head. The 
L/S 16 tubing has an outer diameter of $6.0 \mathrm{~mm}$ and an inner diameter of $3.1 \mathrm{~mm}$. This tubing was selected for use in the pulsatile BVM system for its ability to produce the desired flow rates (highlighted in blue in Table 2.5 at the corresponding desired pulse rates).

\section{Actual Flow Rate vs. Pump Speed}

The results of the flow rate tests can be seen below in Table 2.6 and Figure 2.9. For a comparison of the estimated and actual flow rates see Figure 2.10. Due to the larger diameter of the pump tubing used in the pulsatile design, a much lower RPM was required in the pulsatile setup to produce desired flow rate. This was desirable as it produced appropriate pulse rates that corresponded to physiologic conditions as compared to the high pulse rates using the 8-roller head.

Table 2.6 Actual flow rates of steady and pulsatile flow configurations.

\begin{tabular}{|c|c|c|c|c|c|}
\hline \multicolumn{6}{|c|}{ Measured Flow Rates for 3-roller Pump Head } \\
\hline Pump RPM & $\begin{array}{c}\text { Flow Rate } \\
(\mathrm{ml} / \mathrm{min})\end{array}$ & $\begin{array}{c}\text { Pulse Rate } \\
(\mathrm{bpm})\end{array}$ & Pump RPM & $\begin{array}{c}\text { Flow Rate } \\
(\mathrm{ml} / \mathrm{min})\end{array}$ & $\begin{array}{c}\text { Pulse Rate } \\
(\mathrm{bpm})\end{array}$ \\
\hline 10 & 9 & 30 & 30 & 27.5 & 90 \\
\hline 15 & 14 & 45 & 35 & 32 & 105 \\
\hline 20 & 18.5 & 60 & 40 & 36 & 120 \\
\hline 25 & 22.8 & 75 & 45 & 41 & 135 \\
\hline
\end{tabular}

\begin{tabular}{|c|c|c|c|c|c|}
\hline \multicolumn{7}{|c|}{ Measured Flow Rate for 8-roller Pump Head } \\
\hline Pump RPM & $\begin{array}{c}\text { Flow Rate } \\
(\mathrm{ml} / \mathrm{min})\end{array}$ & $\begin{array}{c}\text { Pulse Rate } \\
(\mathrm{bpm})\end{array}$ & Pump RPM & $\begin{array}{c}\text { Flow Rate } \\
(\mathrm{ml} / \mathrm{min})\end{array}$ & $\begin{array}{c}\text { Pulse Rate } \\
(\mathrm{bpm})\end{array}$ \\
\hline 100 & 10 & 800 & 220 & 23 & 1760 \\
\hline 110 & 11 & 880 & 240 & 24.5 & 1920 \\
\hline 120 & 11.5 & 960 & 260 & 27 & 2080 \\
\hline 130 & 13 & 1040 & 280 & 29 & 2240 \\
\hline 140 & 15 & 1120 & 300 & 30.5 & 2400 \\
\hline 150 & 16 & 1200 & 320 & 32.5 & 2560 \\
\hline 160 & 17 & 1280 & 340 & 34 & 2720 \\
\hline 170 & 18 & 1360 & 360 & 36.5 & 2880 \\
\hline 180 & 19 & 1440 & 380 & 38.5 & 3040 \\
\hline 190 & 20 & 1520 & 400 & 40 & 3200 \\
\hline 200 & 21 & 1600 & & & \\
\hline
\end{tabular}




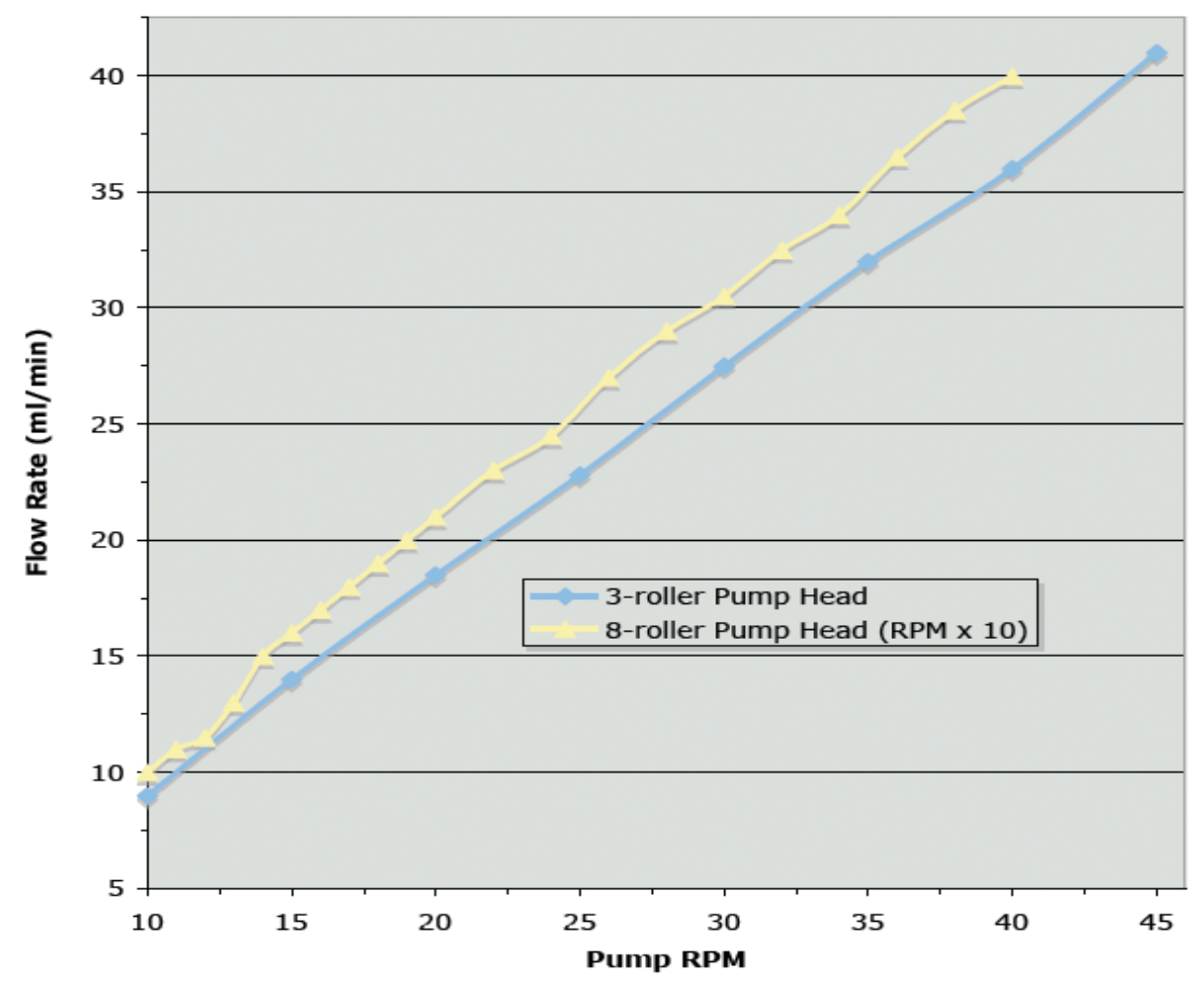

Figure 2.9 Plot of actual flow rates of 3 and 8 roller pump heads in Table 2.6.

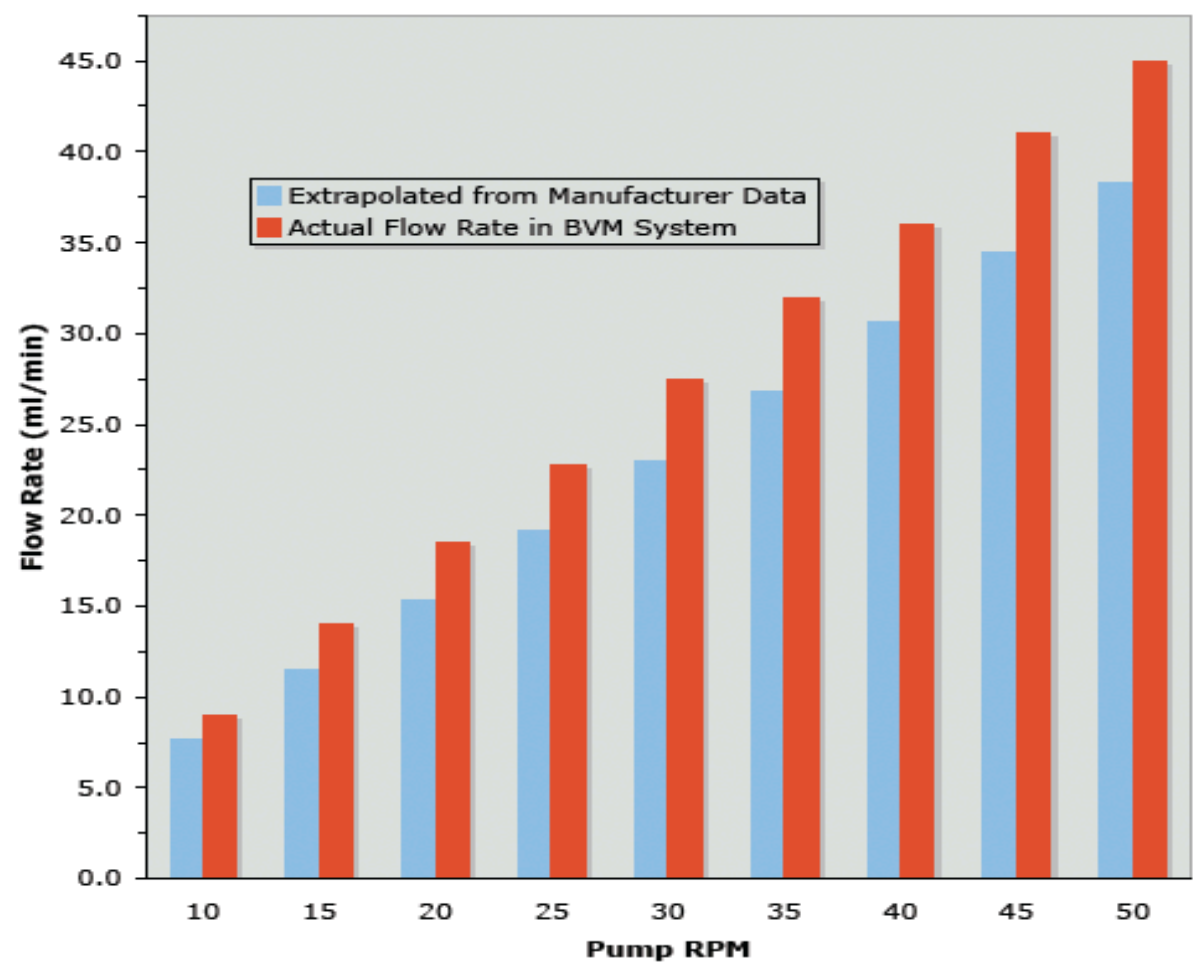

Figure 2.10 Estimated vs. actual flow rates using 3-roller pump head. 
Although the actual flow rates were higher than those predicted using the manufacturers data for L/S 16 tubing, they were relatively close, demonstrating that not only does the system produce appropriate flow rates at desired pulse rates, but also the decision to use L/S 16 tubing was appropriate.

\section{$\underline{\text { Pressure Fluctuation Tests }}$}

\section{Characterization of Original Steady Flow Configuration}

As expected, the small tubing and 8-roller pump head produced a pressure profile with consistently small fluctuations at very high pulse rates (Figure 2.11). The same setup was then tested using growth media in the system instead of water. Although this waveform is not shown, the use of media did not alter the resulting pressure profile.

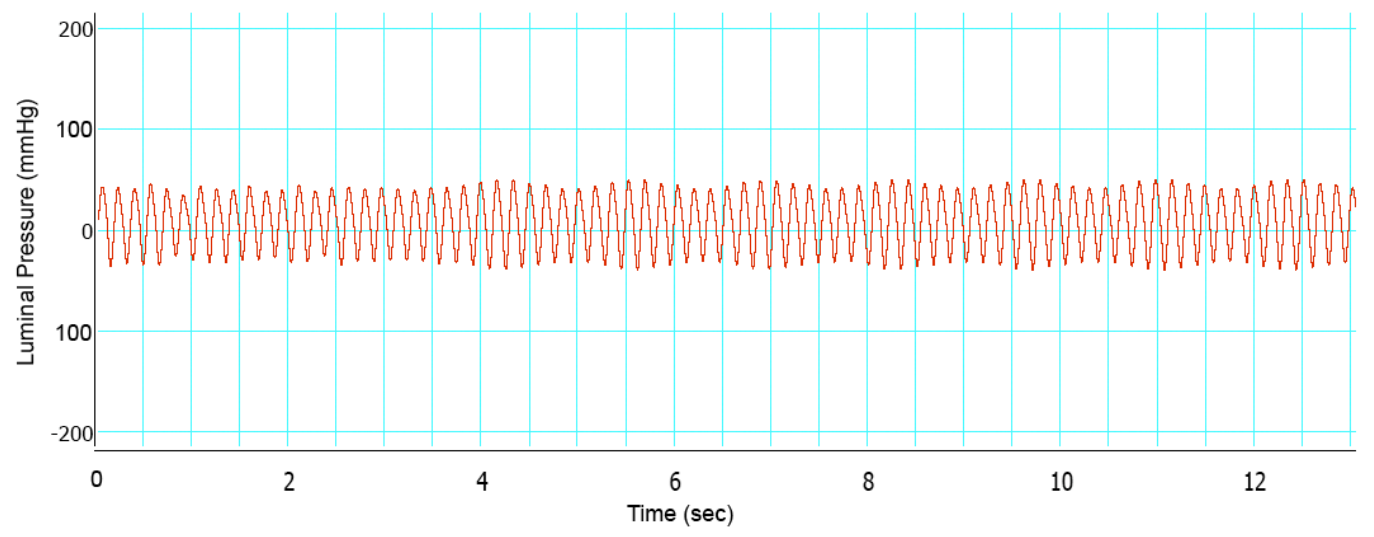

\section{Figure 2.11 Pressure profile of steady flow setup at $\sim 11 \mathrm{ml} / \mathrm{min}$.}

The steady flow setup was tested at different pump speeds to determine how the luminal pressure was affected. There was an observed increase in pressure levels in the system. However, this increase was not uniform, with a sharp decrease seen at higher RPMs, an issue possibly due to cavitations in the tubing at the pump head (Table 2.7 and Figure 2.12). 
Table 2.7 Measured flow characteristics of steady flow configuration.

\begin{tabular}{|c|c|c|c|c|c|}
\hline \multicolumn{5}{|c|}{ Flow Rate and Pressure Figures for 8-roller Pump Head } \\
\hline $\begin{array}{c}\text { Pump } \\
\text { RPM }\end{array}$ & $\begin{array}{c}\text { Flow Rate } \\
(\mathrm{ml} / \mathrm{min})\end{array}$ & $\begin{array}{c}\text { Maximum } \\
\text { Pressure } \\
(\mathrm{mmHg})\end{array}$ & $\begin{array}{c}\text { Minimum } \\
\text { Pressure } \\
(\mathrm{mmHg})\end{array}$ & $\begin{array}{c}\text { Pressure } \\
\text { Differential } \\
(\mathrm{mmHg})\end{array}$ & $\begin{array}{c}\text { Pulse Rate } \\
\text { (bpm) }\end{array}$ \\
\hline 100 & 10 & 16 & 8 & 8 & 800 \\
\hline 110 & 11 & 17 & 7 & 10 & 880 \\
\hline 120 & 11.5 & 18 & 6 & 12 & 960 \\
\hline 130 & 13 & 20 & 5 & 15 & 1040 \\
\hline 140 & 15 & 22 & 5 & 17 & 1120 \\
\hline 150 & 16 & 25 & 2 & 23 & 1200 \\
\hline 160 & 17 & 27.5 & 0.7 & 26.8 & 1280 \\
\hline 170 & 18 & 28 & 1 & 27 & 1360 \\
\hline 180 & 19 & 28 & 0.5 & 27.5 & 1440 \\
\hline 190 & 20 & 30 & 2.8 & 27.2 & 1520 \\
\hline 200 & 21 & 28 & 1.2 & 26.8 & 1600 \\
\hline 220 & 23 & 29 & 0.7 & 28.3 & 1760 \\
\hline 240 & 24.5 & 31 & -2.5 & 33.5 & 1920 \\
\hline 260 & 27 & 33 & -3 & 36 & 2080 \\
\hline 280 & 29 & 40 & -3 & 43 & 2240 \\
\hline 300 & 30.5 & 46 & -4 & 50 & 2400 \\
\hline 320 & 32.5 & 53 & -3.8 & 56.8 & 2560 \\
\hline 340 & 34 & 50 & -4 & 54 & 2720 \\
\hline 360 & 36.5 & 47 & -5 & 52 & 2880 \\
\hline 380 & 38.5 & 46 & -5 & 51 & 3040 \\
\hline 400 & 40 & 41 & -6 & 47 & 3200 \\
\hline
\end{tabular}

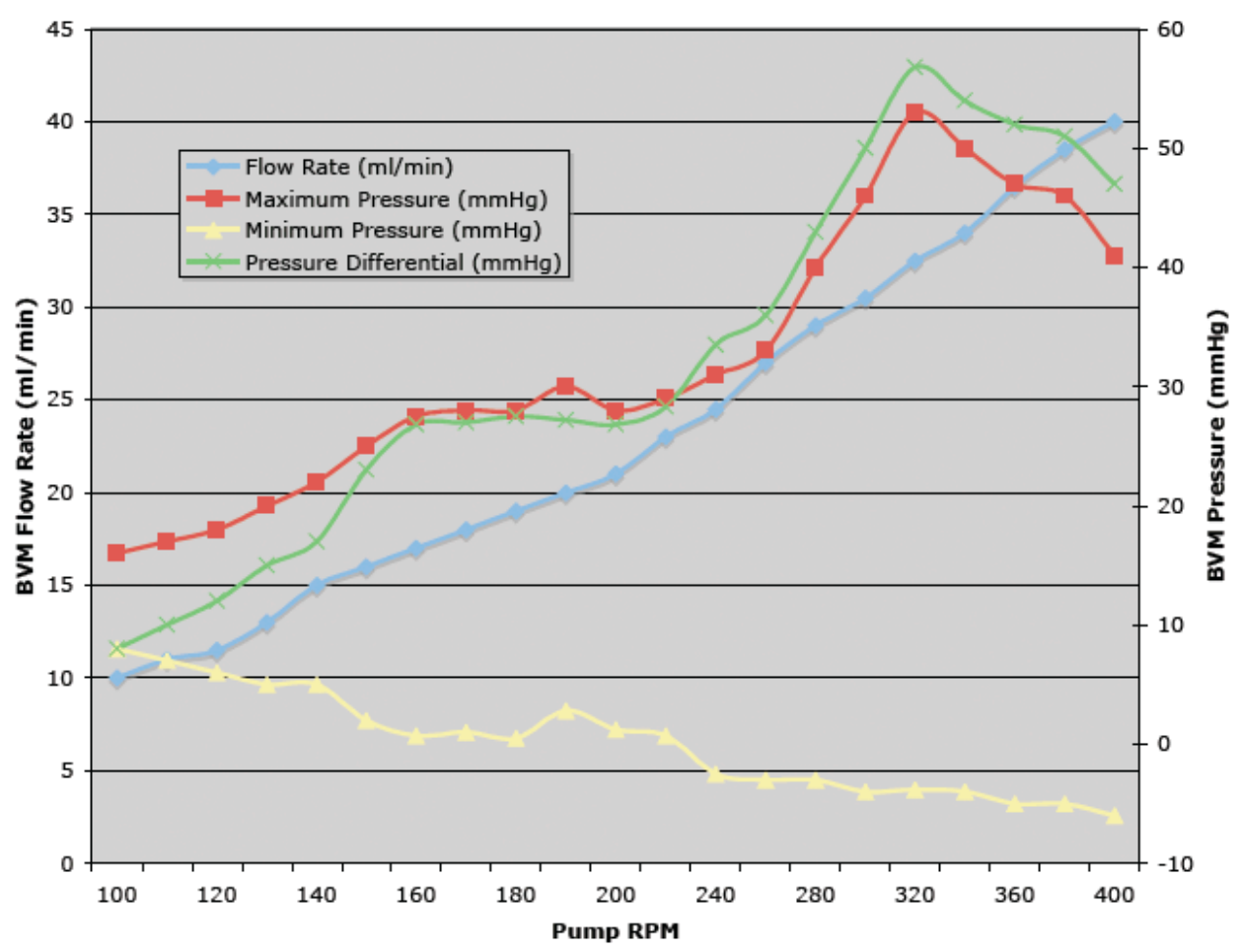

Figure 2.12 Plot flow characteristics of steady flow setup with 8-roller pump from data in Table 2.7 . 


\section{Characterization of Pulsatile Setup}

\section{Initial Testing with Closed Loop}

The initial testing of the pulsatile setup with the 3-roller pump head and simple closed loop of L/S 16 tubing indicated the presence of a vacuum following the positive pressure spike. This was remedied by the addition of the media reservoir to the system between the pump and stopcock (Figure 2.13).
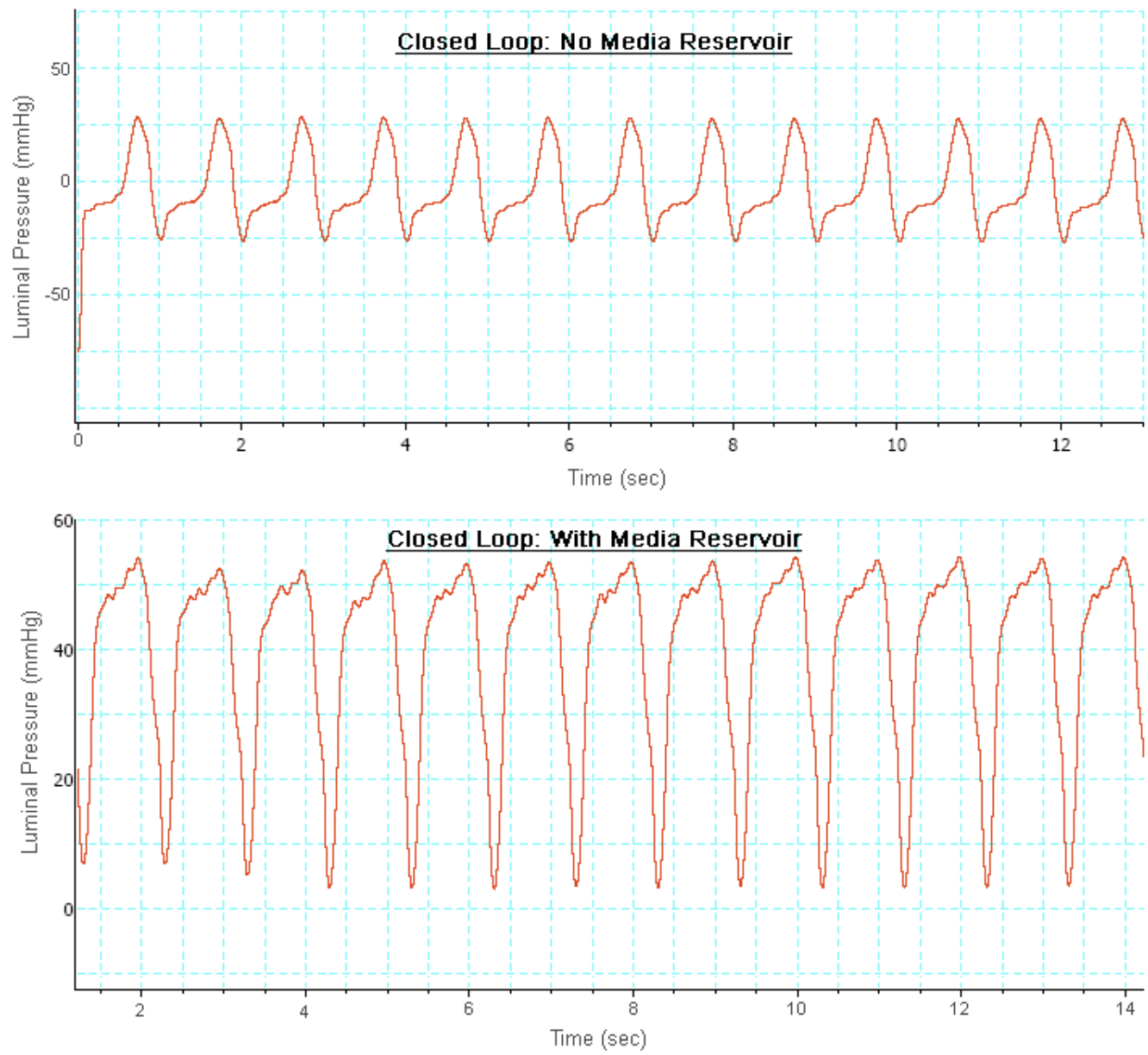

Figure 2.13 Pressure profiles of closed loop with 3 -roller pump at $\sim 18.5 \mathrm{ml} / \mathrm{min}$ using a ratchet clamp downstream of pressure transducer to create backpressure. Note the presence of a vacuum in top graph that was remedied by the addition of the media reservoir as seen in the bottom profile. 


\section{Addition of BVM Chamber}

Pressure profiles were obtained following the addition of the BVM chamber to the closed loop setup. This setup showed similar results to the simple loop with the reservoir (Figure 2.14).

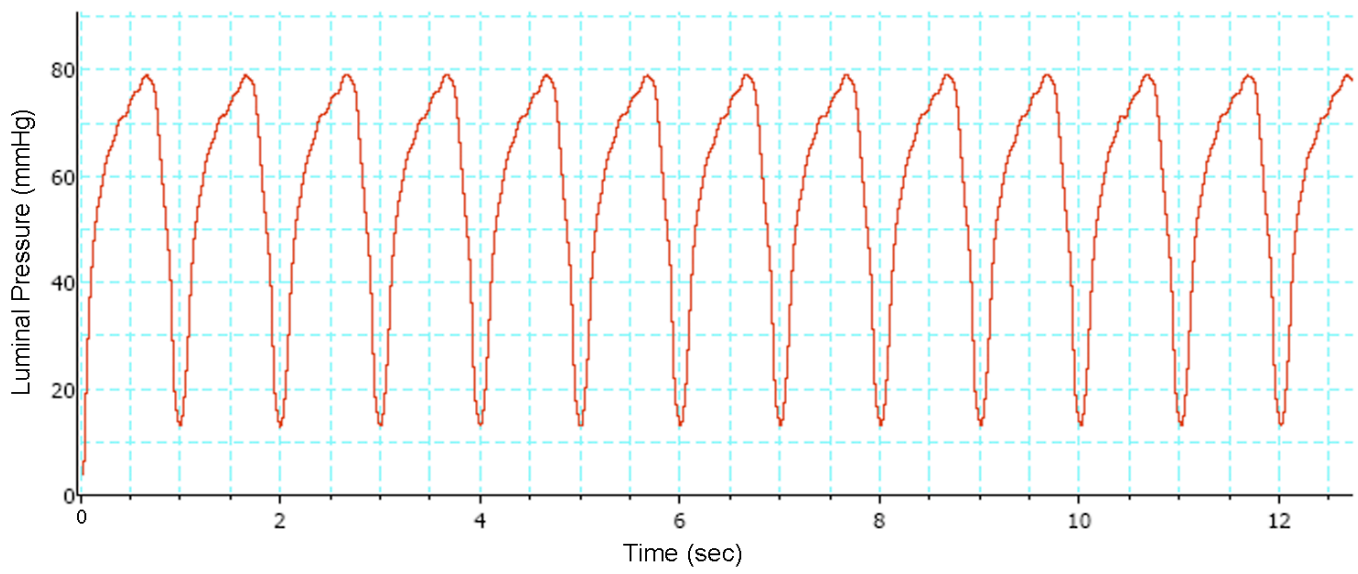

Figure 2.14 Pressure profile of system with BVM chamber and 3-roller pump at $\sim 18.5 \mathrm{ml} / \mathrm{min}$ with ratchet clamp downstream of the pressure transducer to create backpressure.

\section{System Pressure with Water vs. Media}

The complete system was then tested with water and media using backpressure and pump speeds setting to ensure that the use of media would not significantly alter the pressure profile of the system. There was no observed change caused by the introduction of media to the system (Figure 2.15). 


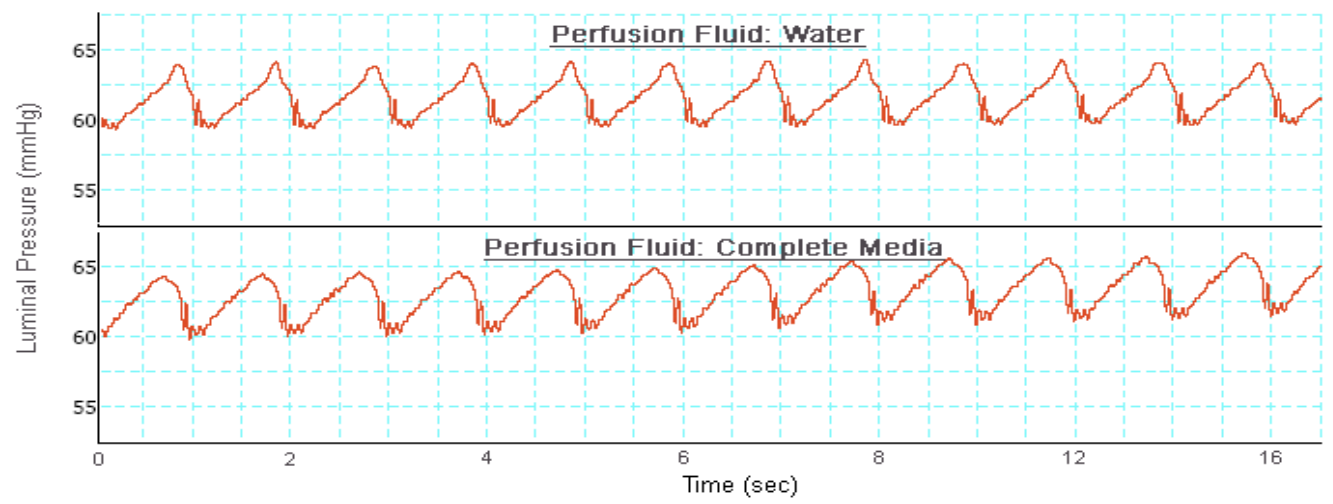

Figure 2.15 Comparison of perfusion fluids in complete pulsatile system at $\sim 18.5$ $\mathrm{ml} / \mathrm{min}$.

\section{Establishing Back Pressure}

Use of the roller clamp provided similar results upon initial testing and identical pressure waveforms as those obtained with the stopcock. However, when an attempt was made to establish settings for various flow models, the roller clamp posed similar difficulty in allowing for small variations in pressure. Although this was an improvement over the stopcock, the ratchet clamp was investigated as a means of improving the ability to fine-tune the pressure settings. The ratchet clamp proved to be the most user-friendly of the three, however, adjustment of the pressure ranges were still not as indexable as desired as very small adjustments of the clamp caused large changes in system pressure. See Figure 2.16 for the tested devices. As a result, a single applicable clamp setting was set using the pressure measurement system and desired pressure and flow was adjusted via pump speed only. 

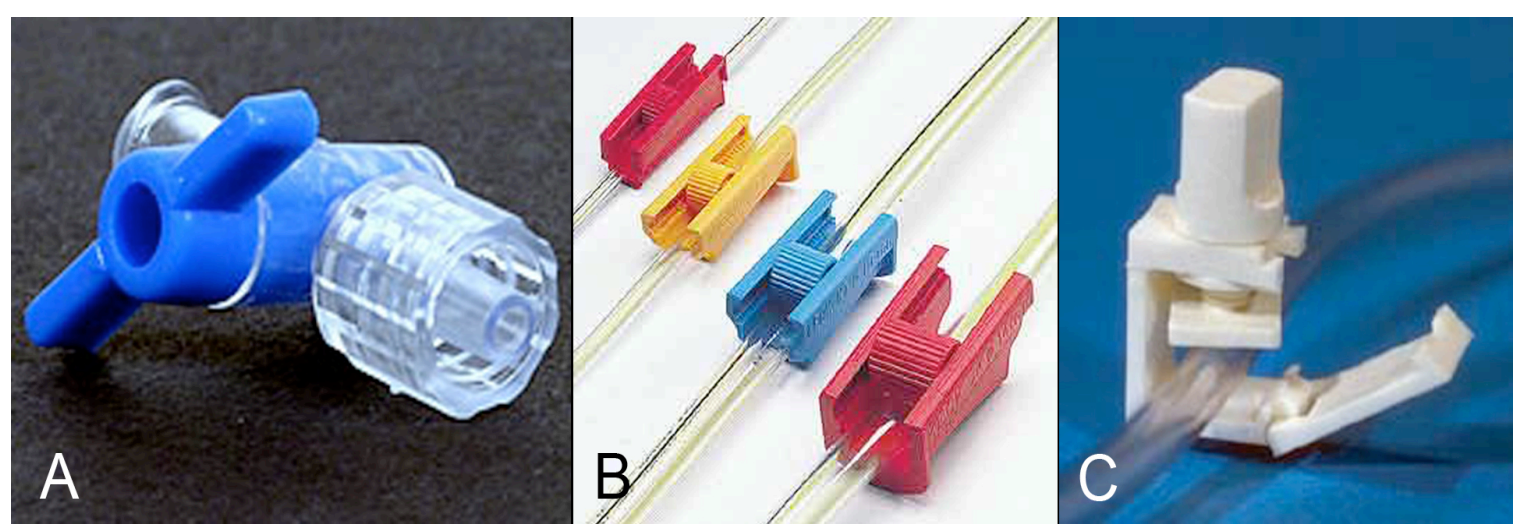

Figure 2.16 Tested options for creating backpressure. (A) Stopcock, (B) Roller Clamp, (C) Ratchet clamp.

The flow rates and pressure readings were measured in the pulsatile setup to determine the pressure endpoints at different pump speeds. Although pump speed and pressure can be adjusted independently in this system, the backpressure was left constant during these tests in order to simplify the setup. Contrary to the steady flow setup using the 8-roller pump head, luminal pressure increased steadily relative to pump speed (Table 2.8) and with the backpressure setting left unchanged, the system pressure increased with pump speed, but the measure flow rates and waveforms remained similar (Table 2.8 and Figure 2.17).

Table 2.8 Measured flow characteristics of complete pulsatile system with ratchet clamp in place.

\begin{tabular}{|c|c|c|c|c|c|}
\hline \multicolumn{5}{|c|}{ Flow Rate and Pressure Figures for 3-roller Pump Head } \\
\hline $\begin{array}{c}\text { Pump } \\
\text { RPM }\end{array}$ & $\begin{array}{c}\text { Flow Rate } \\
(\mathrm{ml} / \mathrm{min})\end{array}$ & $\begin{array}{c}\text { Maximum } \\
\text { Pressure } \\
(\mathrm{mmHg})\end{array}$ & $\begin{array}{c}\text { Minimum } \\
\text { Pressure } \\
(\mathrm{mmHg})\end{array}$ & $\begin{array}{c}\text { Pressure } \\
\text { Differential } \\
(\mathrm{mmHg})\end{array}$ & $\begin{array}{c}\text { Pulse Rate } \\
(\mathrm{bpm})\end{array}$ \\
\hline 10 & 9 & 27 & 9 & 18 & 30 \\
\hline 15 & 14 & 36 & 5 & 31 & 45 \\
\hline 20 & 18.5 & 79 & 17 & 62 & 60 \\
\hline 25 & 22.8 & 101 & 19 & 82 & 75 \\
\hline 30 & 27.5 & 128 & 33 & 95 & 90 \\
\hline 35 & 32 & 150 & 50 & 100 & 105 \\
\hline 40 & 36 & 166 & 66 & 100 & 120 \\
\hline 45 & 41 & 170 & 69 & 101 & 135 \\
\hline
\end{tabular}




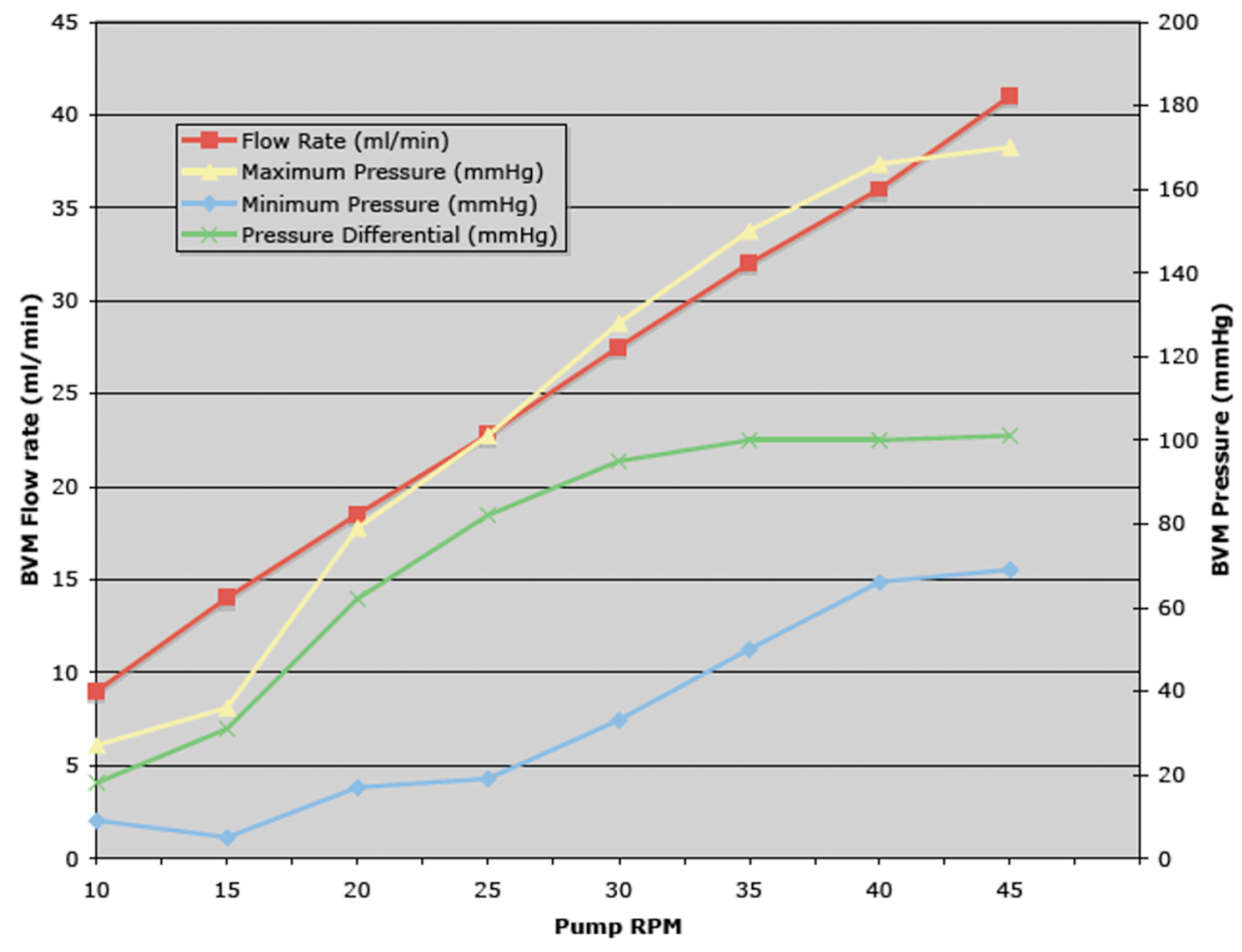

Figure 2.17 Plot of flow characteristics of pulsatile setup from data in Table 2.8. 


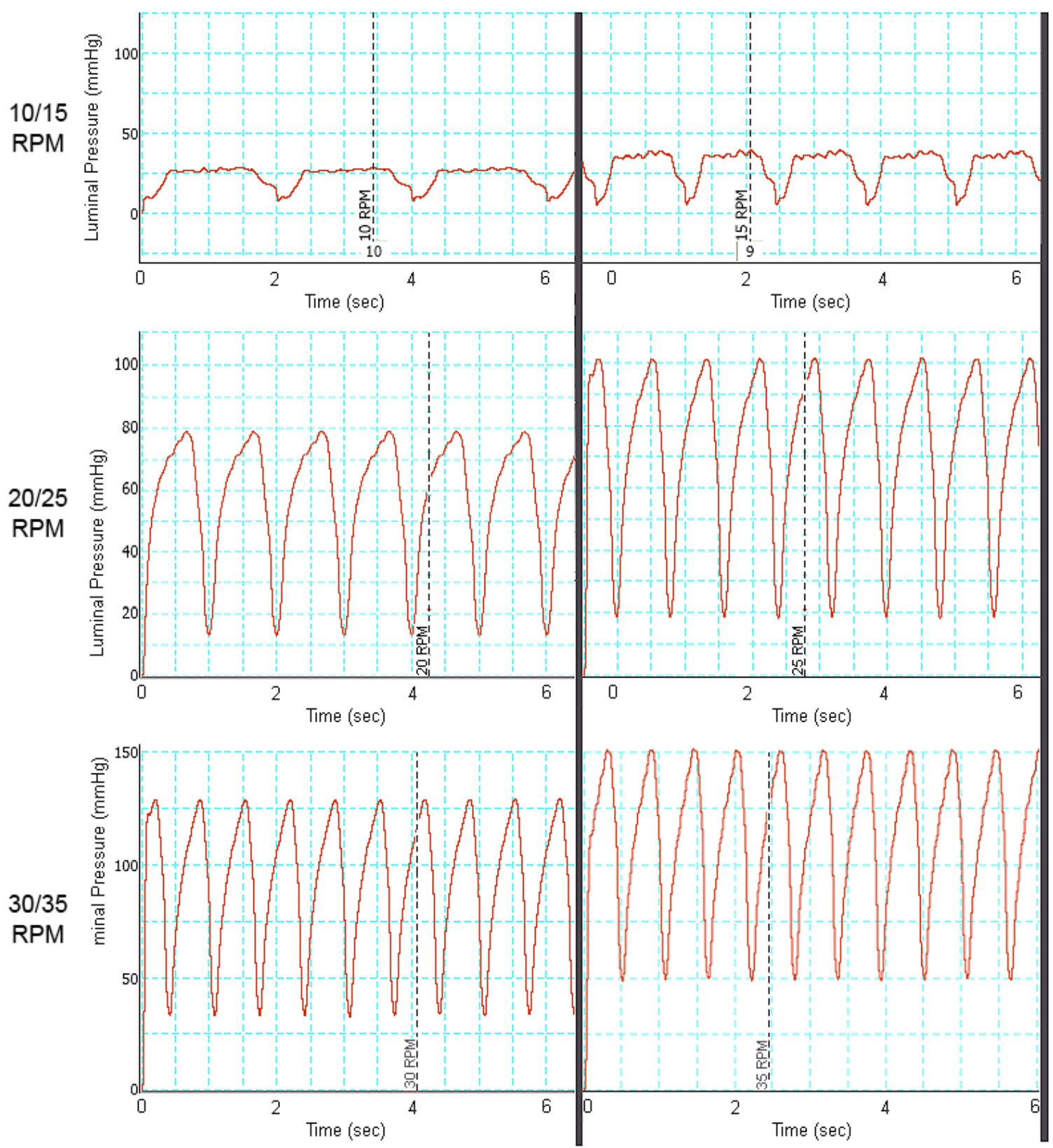

Figure 2.18 Pressure profiles with constant backpressure and varied pump speed.

As seen in the plots above in Figure 2.18, the waveforms of the 10 and 15 RPM settings appear different than the 20-35 RPM settings. These recordings were done using identical system configurations with the only variable being changes in pump speed. The difference in the first two waveforms is most likely time dependent as the increased pressure at these settings had to be maintained over the same period with the flattened, 
wavy peaks indicating that the system pressure was held at the elevated level between pulses.

\section{Pressure Differentials: Cardiac vs. BVM}

BVM pressures were compared to those found as a result of native cardiac outputs in patients with varied degrees of hypertension (Table 2.9 and Figure 2.19). This comparison allows for the use of the BVM as a model of disease conditions by simply increasing the pump speed while leaving the backpressure setting constant. Due to the fact that the BVM system is open and thus cannot maintain a baseline pressure, the minimum pressure, and therefore, the pressure differentials in the BVM system are not significantly similar to native conditions (Figure 2.20). However, the maximum pressures seen in the BVM lumen showed similar increases as the native systolic pressures found in varying degrees of hypertension. This is important, as the peak pressure is responsible for the cyclic wall strain that was previously described as having great importance in vessel development.

Table 2.9 Comparison of cardiac blood pressure conditions and flow settings in pulsatile BVM system (blood pressure figures taken from [80].

\begin{tabular}{|c|c|c|c|c|c|c|}
\hline $\begin{array}{c}\text { Cardiac Blood Pressure } \\
\text { Condition/Pump RPM }\end{array}$ & $\begin{array}{c}\text { Maximum } \\
\text { BVM Pressure } \\
(\mathrm{mmHg})\end{array}$ & $\begin{array}{c}\text { Minimum } \\
\text { BVM Pressure } \\
(\mathrm{mmHg})\end{array}$ & $\begin{array}{c}\text { BVM Pressure } \\
\text { Differential } \\
(\mathrm{mmHg})\end{array}$ & $\begin{array}{c}\text { Systolic } \\
\text { Cardiac } \\
\text { Pressure } \\
(\mathrm{mmHg})\end{array}$ & $\begin{array}{c}\text { Diastolic } \\
\text { Cardiac } \\
\text { Pressure } \\
(\mathrm{mmHg})\end{array}$ & $\begin{array}{c}\text { Cardiac } \\
\text { Pressure } \\
\text { Differential } \\
(\mathrm{mmHg})\end{array}$ \\
\hline Normal/10 RPM & 27 & 9 & 18 & 90 & 60 & 30 \\
\hline Normal/15 RPM & 36 & 5 & 31 & 100 & 65 & 35 \\
\hline Normal/20 RPM & 79 & 17 & 62 & 110 & 70 & 40 \\
\hline Normal/25 RPM & 101 & 19 & 82 & 120 & 80 & 40 \\
\hline Normal/30 RPM & 128 & 33 & 95 & 130 & 85 & 45 \\
\hline Normal/High & N/A & N/A & N/A & 140 & 90 & 50 \\
\hline Mild High/35 RPM & 150 & 50 & 100 & 150 & 95 & 55 \\
\hline Moderate High/40 RPM & 166 & 66 & 100 & 160 & 100 & 60 \\
\hline Moderate High/45RPM & 170 & 69 & 101 & 170 & 105 & 65 \\
\hline
\end{tabular}




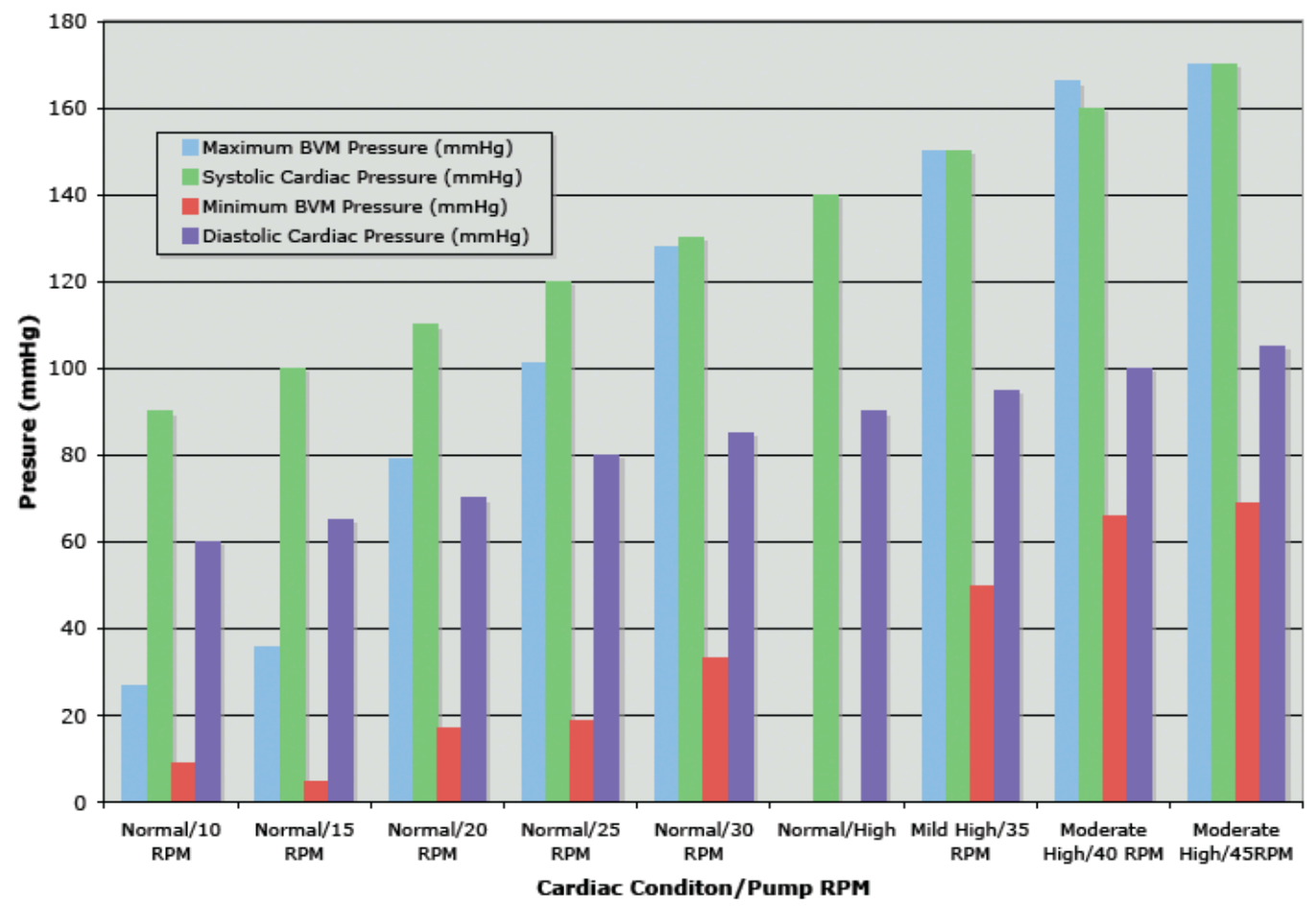

Figure 2.19 Comparison of BVM vs. native cardiac pressures.

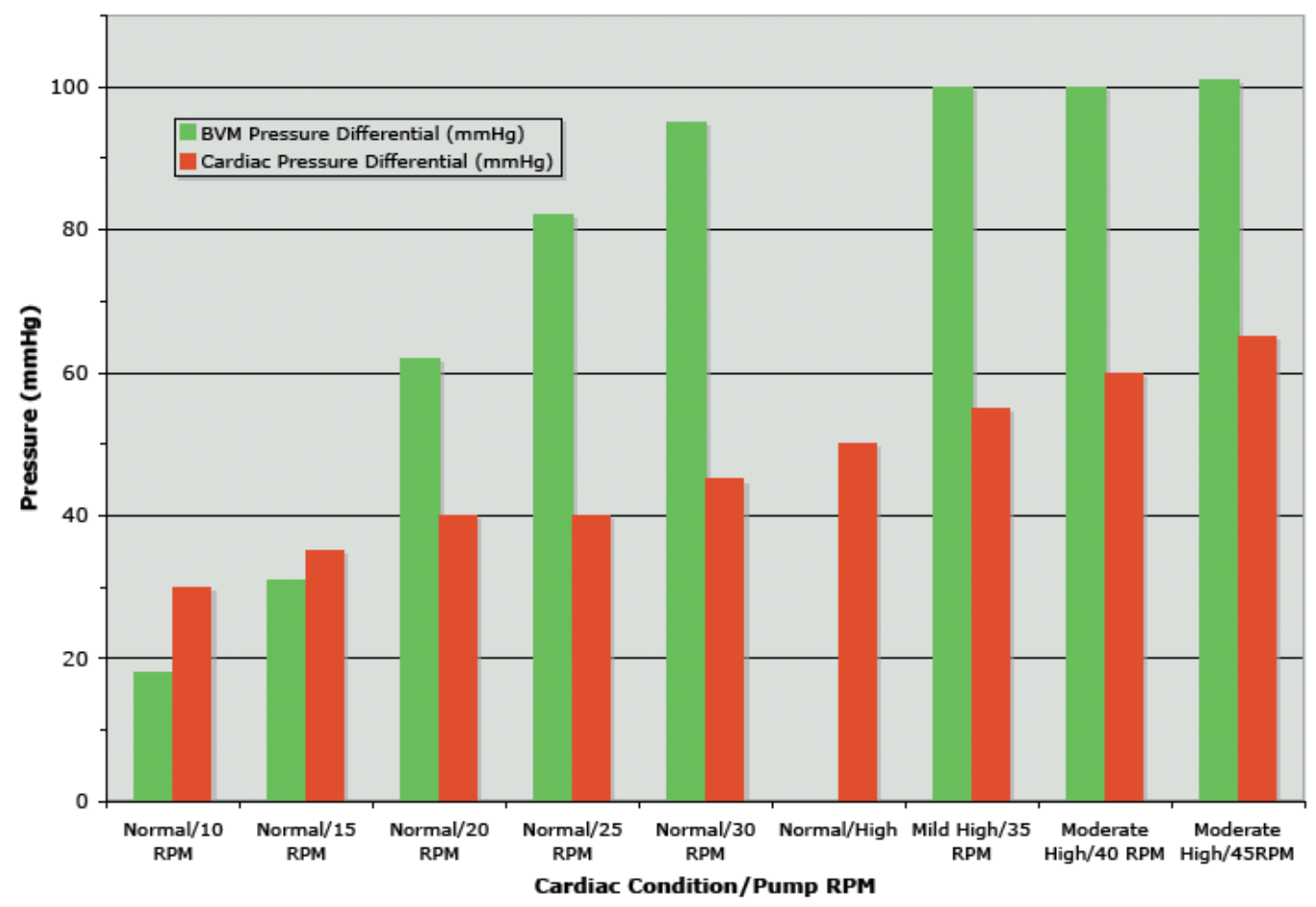

Figure 2.20 Comparison of BVM vs. native cardiac pressure differentials. 


\section{Preliminary Cell Adhesion Test}

After running a HUVSMC-sodded vessel for several minutes under pulsatile flow, it was noticed that the fluid level in the media reservoir was dropping, indicating a problem with the return flow. Upon examination, it was assumed that because pressurization and pulsation relies on restricting luminal flow, the increased pressure seen at the porous graft was resulting in transmural flow rather than maintaining pulsatile luminal flow. It was assumed that this might be a result of the compressible air in the headspace of the BVM chamber.

Since no immediate solution was possible without disrupting the system, the pressure and pump speed were reduced to $\sim 30 \mathrm{mmHg}$ and $15 \mathrm{RPM}$ in an effort to allow relief of the backpressure via luminal flow only. After running for several minutes at these new settings, there was no apparent drop in the media level in the reservoir. One of the BVM setups was taken down after running for 2 hours under pulsatile flow at $\sim 30 \mathrm{mmHg}$ to allow for assessment of the construct after exposure to pulsatile flow for a relatively short time period.

The second of the two setups was maintained at $\sim 30 \mathrm{mmHg}$ and 15 RPM overnight. Because the empty headspace of the BR chamber was thought to be a cause of the excessive transmural flow, it was filled with media for the overnight run. The next morning it was noticed that the level of the media reservoir had dropped below the draw tube and the tubing of the BVM system and graft did not contain any media. A leak was discovered that later was determined to be a breach in the seal of the lid of the BVM chamber. It is unknown how long the system was in this state. When this leak was discovered this setup was immediately taken down. 
All grafts taken down following this preliminary test were placed in Histochoice to preserve them. However, due to the problems experienced with excessive transmural flow and leaking, these samples were not analyzed as it was assumed that the resulting data could not be used to draw any valuable conclusions.

\section{Follow-up Pressure Tests}

The systems were retested on the AD Instruments LabChart system and it was determined that the pressurization of the extra-luminal chamber was caused by backflow through the extra-luminal outlet. This was remedied by placing a tubing clamp on the outlet tubing that could be tightened following the sodding procedure. The tests with the denucleated graft showed no change in waveforms or the ability to sustain pressure. As an additional check, the system was run at target pressures with no fluid in the extraluminal space to visually assess the degree of transmural flow. No transmural flow was detected under these conditions. As a final confirmation of the system's ability to maintain consistent pressure over extended periods of time, the system was configured and ran as it would be for pulsatile graft cultivation while monitoring system pressure with the ADI system for 4 hours. Although this result is not shown, there was little variation in system pressure from the beginning to the end of the test.

\section{Final Cell Adhesion Tests}

The setup of the six BVM constructs proceeded without difficulty. Each graft was sodded with approximately $7 \times 10^{6}$ cells, providing a sodding density of $\sim 1.6 \times 10^{6}$ cells $/ \mathrm{cm}^{2}$. When inserting the graft into the chambers of constructs PA1, PB3 and PB4, it 
was noticed that the graft had to be somewhat stretched causing it to be under tension once in place. It was unknown how this would affect the sodding or cultivation. See Figure 2.21 to understand construct name codes.

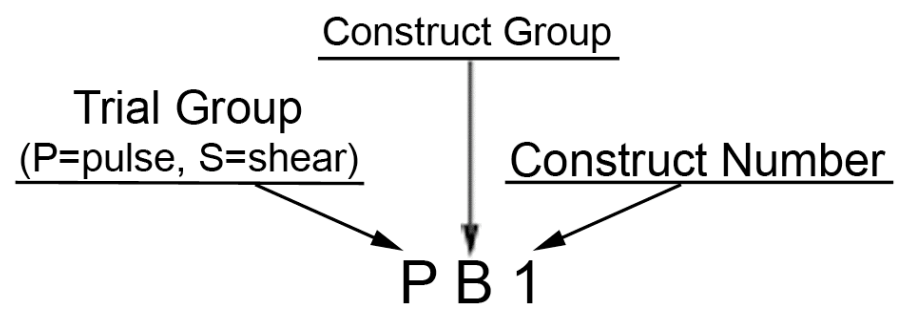

Figure 2.21 Explanation of construct naming scheme.

The initial 72 -hour growth period under steady flow conditions at $15 \mathrm{ml} / \mathrm{min}$ was completed without any major complications and after 72 hours, the variable groups were transferred to the 3-roller pumps and the systems were pressurized to initiate pulsatile flow under the conditions outlined in Table 2.3. When changing construct PB3 over to pulsatile flow, it was noticed that the small diameter pump tubing used in the initial steady flow growth period had developed a minor crack resulting in a slight leak. This did not result in any significant loss of media volume. The complications experienced when pressurizing the system in the preliminary cell adhesion tests were not seen in this trial, allowing all five of the pressurized variable groups to run for 24 hours without any problems. In order to achieve the desired pulsation pressure, the backpressure clamp had to be carefully adjusted while observing the media return flow and the resulting clamp settings were consistent among all four of the pressurized systems.

After 24 hours of pulsatile flow, all bioreactor systems were taken down. Before removing the bioreactor systems from the incubator it was noticed that PA1 and PB4 had developed significant airspace (bubbles) in the graft that may have prevented the dorsal 
(upper) hemisphere of the graft from being fully immersed in media. It is unknown how long this condition existed.

BBI imaging showed that cells remained on all six grafts following exposure to steady flow as well as the varying levels of pulsation (Figure 2.22-Figure 2.25). This was confirmed by SEM imaging and although all constructs indicated the presence of cells on the scaffold, the number of cells present varied. For purposes of the analysis presented here, cell coverage on the grafts was assessed on a qualitative basis by estimating coverage as percentage of the overall surface.

Analysis of construct PA1, the 8-roller steady-flow control setup, indicated that cells were present following the four-day cultivation period, however surface density was not as extensive as expected (Figure 2.22). This result may have been due to the tension under which the graft was installed in the bioreactor. BBI imaging of this construct indicated a considerable number cell nuclei present. However, when visualized under SEM, the cell density on the graft surface did not correspond with this result, as surface coverage was somewhat sparse. There was, however, some indication of cell spreading and/or matrix deposition by the cells that remained on the surface. 


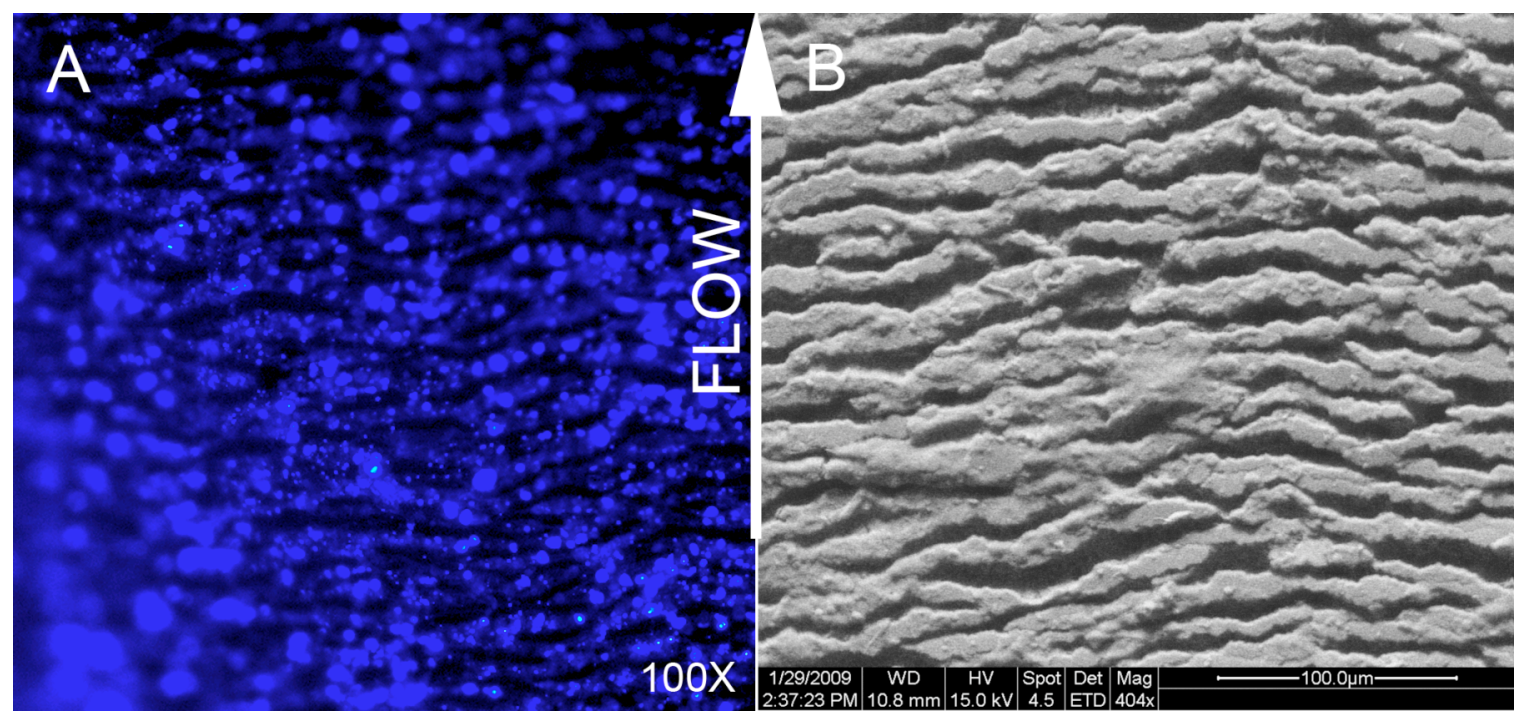

Figure 2.22 BBI(A) and SEM(B) imaging of construct PA1 (96 hours of steady flow (a) $15 \mathrm{ml} / \mathrm{min})$.

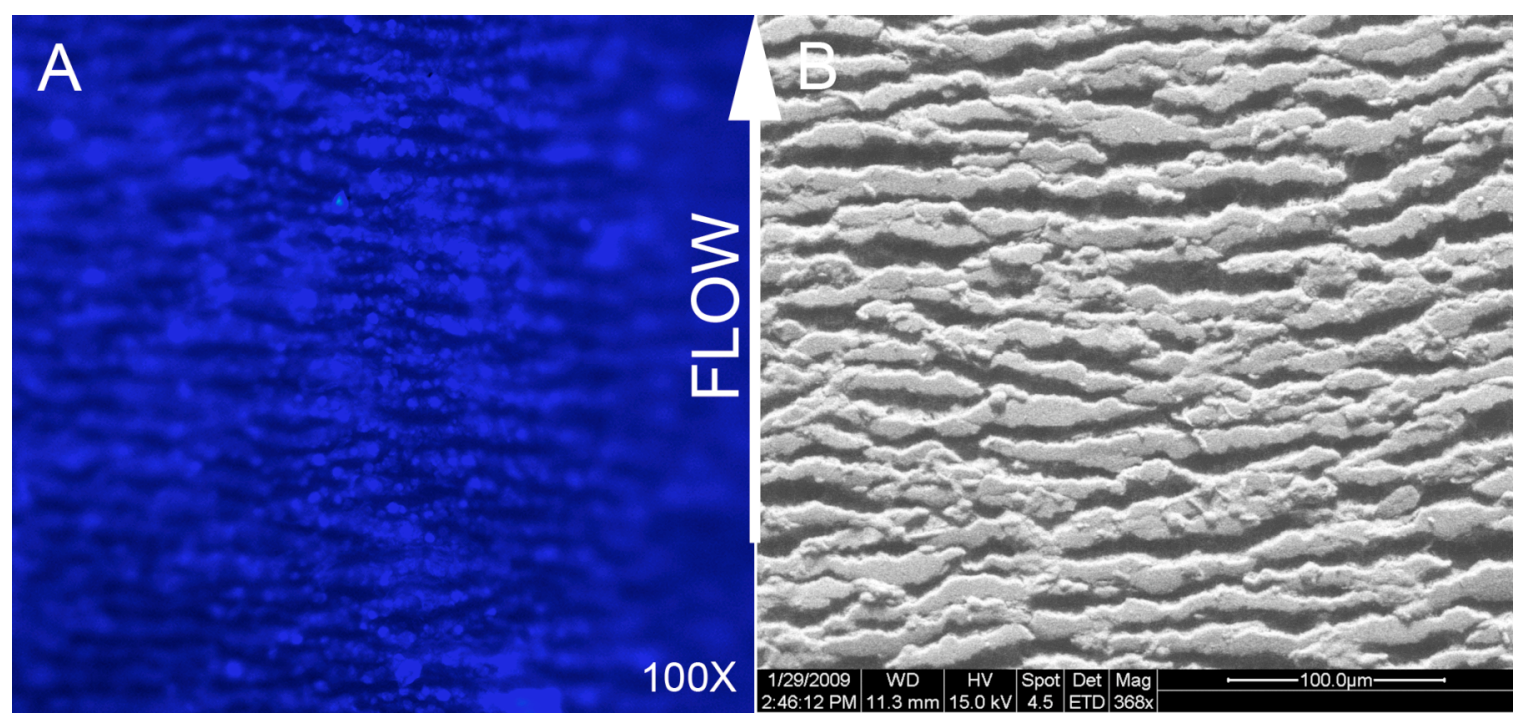

Figure 2.23 BBI(A) and SEM(B) imaging of construct PB1 (72 hours of steady flow on 8-roller pump@ 15ml/min and 24 hours of steady flow on 3-roller pump).

The 3-roller control setup, construct PB1, produced a graft with an increased surface cell density compared to the PA1 construct. The BBI results in Figure 2.23 show a similar number of nuclei to those in Figure 2.22, but there was a moderate increase in the number of cells on and in between the nodes of the ePTFE graft of the PB1 construct (Figure 2.23). 
Constructs grown under 24 hours of pulsation indicated an increased surface cell density as well as more extensive cell extension and/or matrix deposition. This was most evident in construct PB2, which was exposed to $\sim 40 \mathrm{mmHg}$ pulsatile flow for 24 hours. As seen in Figure 2.24, there were more, larger cell nuclei than those in the control construct PA1 and PB1. The surface cell density was also noticeably higher in the SEM image of PB2 and there was an apparent increase in cell extension and/or matrix deposition as indicated by the fibrous tendrils extending across the nodes.

Imaging of the constructs grown under $80 \mathrm{mmHg}$ pulsatile flow indicated that the cells remained on the graft following 24 hours of exposure to the increased level of pulsation (Figure 2.25). The BBI image of the PB5 construct showed a similar number of nuclei as the control groups, however there was a noticeable increase in surface cell density and cell extension and/or matrix deposition as seem in the SEM image. For more construct images See Appendix A.
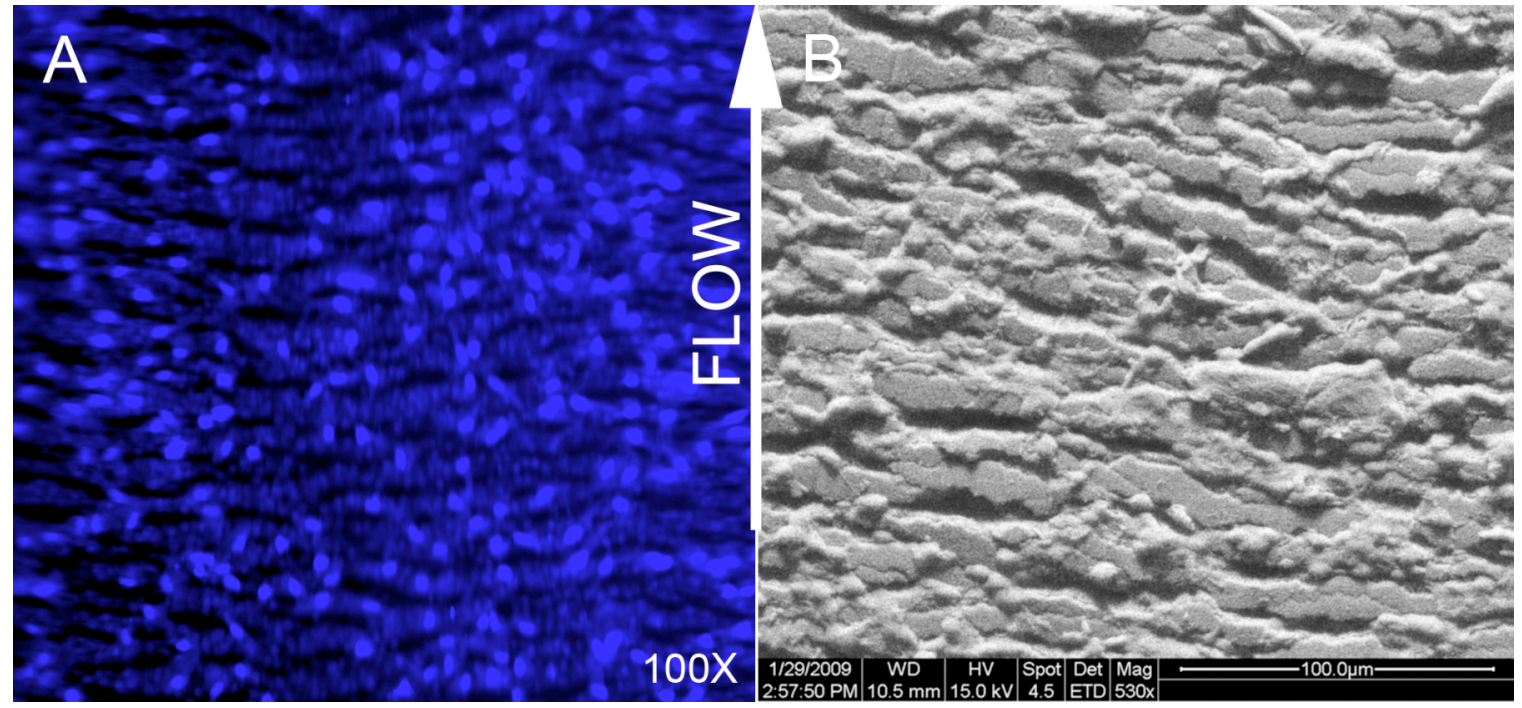

Figure 2.24 BBI(A) and SEM(B) imaging of construct PB2 (72 hours of steady flow on 8-roller pump @ 15ml/min and 24 hours of pulsatile flow on 3-roller pump at $18.5 \mathrm{ml} / \mathrm{min}$ and $\sim 40 \mathrm{mmHg}$ ). 


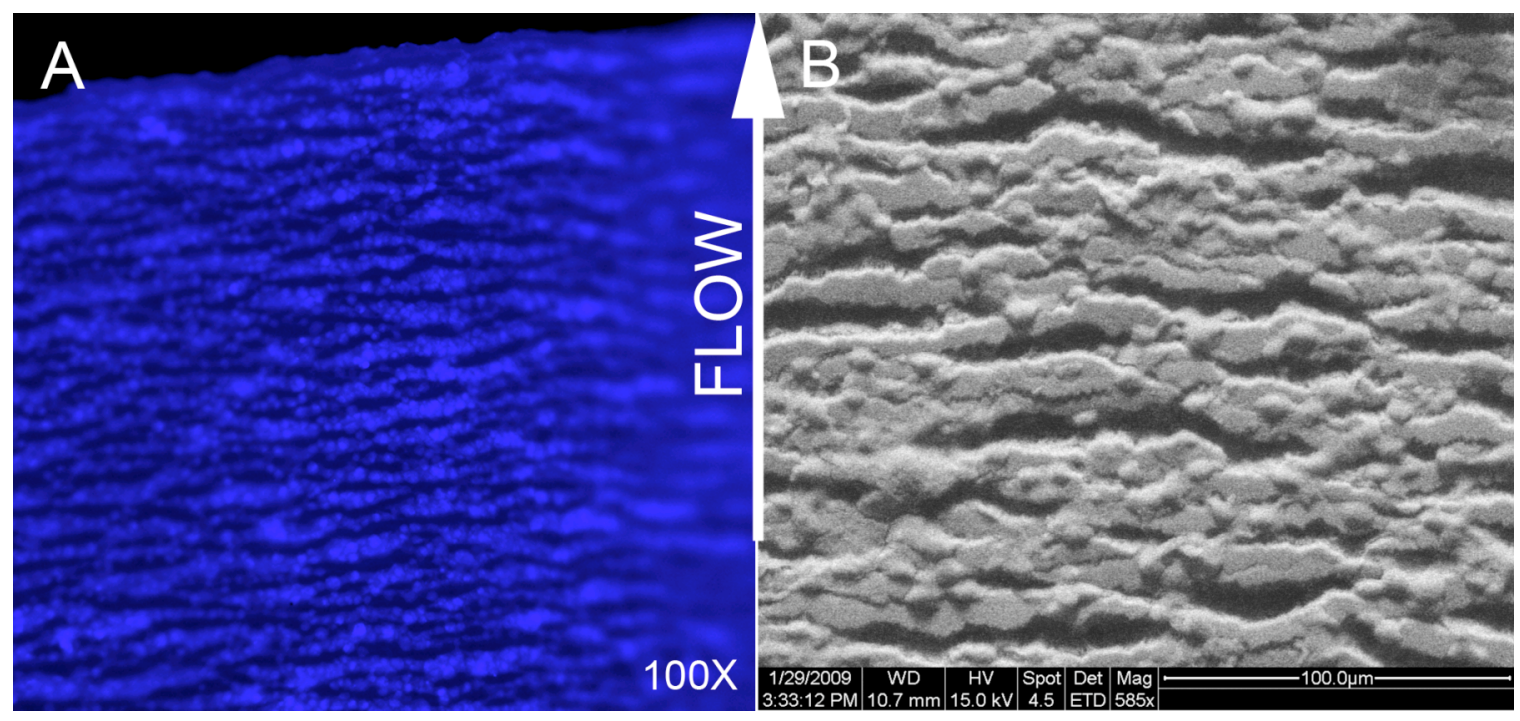

Figure 2.25 BBI(A) and SEM(B) imaging of construct PB5 (72 hours of steady flow on 8-roller pump@ $15 \mathrm{ml} / \mathrm{min}$ and 24 hours of pulsatile flow on 3-roller pump at $18.5 \mathrm{ml} / \mathrm{min}$ and $\sim 80 \mathrm{mmHg}$ ).

\section{DISCUSSION}

\section{Development of Pulsatile System}

The primary aim of the work discussed in this chapter was to develop and implement pulsatile flow in the BVM bioreactor system with the intention of creating flow conditions similar to those experienced by native vessels in order to eventually improve the phenotypic expression of human blood vessel cells grown in vitro. Due to the fact that this system was originally designed to be a simple, high throughput-testing environment for coronary stents, the development of pulsatile flow was approached with the goal of maintaining the original system's simplicity and potential for scale-up. This work was intended to be used as groundwork for the development of an ideal bioreactor system that can be used to produce native-like BVMs. Since this system had not yet been tested under pulsatile conditions, the specific objective of this portion of this thesis was to 
determine if a BVM construct could be cultivated in this system with the addition of pulsatile flow while maintaining cell adhesion.

The original system was configured for steady, perfusive flow via a peristaltic pump that relied on small diameter pump tubing and an eight roller pump head to produce many small pulses to drive flow. Testing of this system demonstrated that the pressure waveform was not desirable as it oscillated between equal positive and negative values at a rate of $\sim 6 \mathrm{~Hz}$ or 360 beats per minute. The initial thought was that pulsatile flow could be established with minor alterations to this previously established system. These changes included using a different pump head with fewer rollers and larger pump tubing; a combination that ultimately produced larger pressure fluctuations at desired flow rates and a lower pulse rate.

To determine which pump head/tubing configuration would provide the desired conditions, the system was tested with a data acquisition system that measured real-time system pressure. This allowed different configurations to be tested and adjusted until a desired waveform was established. The initial choice of the pump head and tubing was based on flow rate and pulse rate. The thought was that if the desired flow rate could be established using a configuration that would concurrently run at a desired pulse rate, the application of backpressure downstream of the growth chamber could be used to create and adjust pressurization of the system. After testing several methods for applying the backpressure, an external tubing clamp was chosen and although this method effectively created desired waveforms, the pressure was not as adjustable as originally desired. This aspect of the system will require further development to allow for more finely tunable system pressure. 


\section{Scalability}

Along with maintaining simplicity, another aspect of the BVM system taken into consideration during the development of pulsatile flow was scalability. The changes made to the system to achieve pulsation did not affect the overall configuration of the system to any great degree as it involved the addition of larger diameter tubing and the use of a different pump head. The only aspect that may affect scalability is the number of

constructs that can be driven by each pump. The original system's eight roller pump head can drive up to twelve systems at once while the 3-roller head configured with the large tubing cartridges used in this study can house only four systems making incubator space more of a concern (See Appendix A).

\section{Comparison to Previous Work}

The implementation of pulsatile flow in a TEVG bioreactor system is not a novel achievement. Although other groups have succeeded in creating systems that accomplish this, the methods used are typically more involved and complex $[28,29,85]$. These systems incorporate multiple pumps, flow meters and flow controllers to create physiologic pulsation. The use of these devices may allow for more finely tunable pressure fluctuations that can be constantly monitoring during cultivation, however the added complexity may also bring about more complications. By establishing pulsatile flow using the methods described in this thesis, there are fewer opportunities for contamination and/or problems that may result from device failure in a large-scale application such as ours. 


\section{Cell Adhesion}

The final step of this work was to determine whether or not a BVM construct could be cultivated under pulsatile flow using the methods described above. As this was preliminary work in establishing pulsatile flow, the primary goal was to find out whether or not the sodded cells would maintain adhesion to the scaffold after exposure to pulsatile pressures. Due to the fact that there was not a large enough sample size to make statistically significant claims, no conclusive statements can be made as to the conditions that will provide optimal growth and cell morphology. However, for purposes of this thesis, it can be concluded that the methods developed here can be used to cultivate a BVM construct under pulsatile flow conditions and that the sodded cells will maintain adhesion to the ePTFE scaffold at peak pressures of $\sim 80 \mathrm{mmHg}$ for up to 24 hours.

Although the cell adhesion test indicated the presence of cells following cultivation, there were some notable observations and assumptions made based on the results. As mentioned above, the surface density of the steady flow control construct (PA1) was unexpectedly low. This was further questioned when the density on the surface of the pressurized variable groups was found to be considerably higher. Because the BBI image of this construct indicates the presence of cell nuclei, the only discrepancy that could explain this result is the tension placed in the scaffold when it was installed in the chamber. The fact that there were nuclei present, but very few cells on the surface may indicate that the stretching of the ePTFE, which was transverse to the nodal orientation of the material, may have expanded the internodal spaces allowing the cells to be pushed deeper in the pores making them indistinguishable under SEM. To determine if this is the case, further analysis should be performed. 


\section{Construct Appearance and Cell Morphology}

While analysis of the cell adhesion test constructs indicated the presence of cells, the resulting morphology when visualized under SEM was questioned, as there was no confluent monolayer that would be expected from cultivation of endothelial cells. Again, the sample size was not large enough to make any substantial claims regarding this result. However, because our lab did not have a great deal of experience culturing HUVECs, the growth characteristics of this cell type were investigated and based on the results of several other groups, there could be several explanations for the resulting morphology that may provide some suggestions for methods used in future work with this cell type. Prior experience with Human Microvessel Endothelial Cells (HMVECs) grown in the BVM system led to the expectation that surface morphology would appear as it does in Figure 2.26. However, a study by Hsu et al into the growth of HUVECs in a perfusion bioreactor resulted in the morphology displayed in Figure 2.27 [31]. When compared to the micrographs of the constructs resulting from the work in this thesis, the morphology is somewhat similar. The Hsu construct was cultivated on a polyurethane scaffold rather than ePTFE which may account for the difference in appearance, however the possibility that the unexpected morphology in our results is a characteristic of the HUVEC phenotype cannot be ruled out without further investigation. 


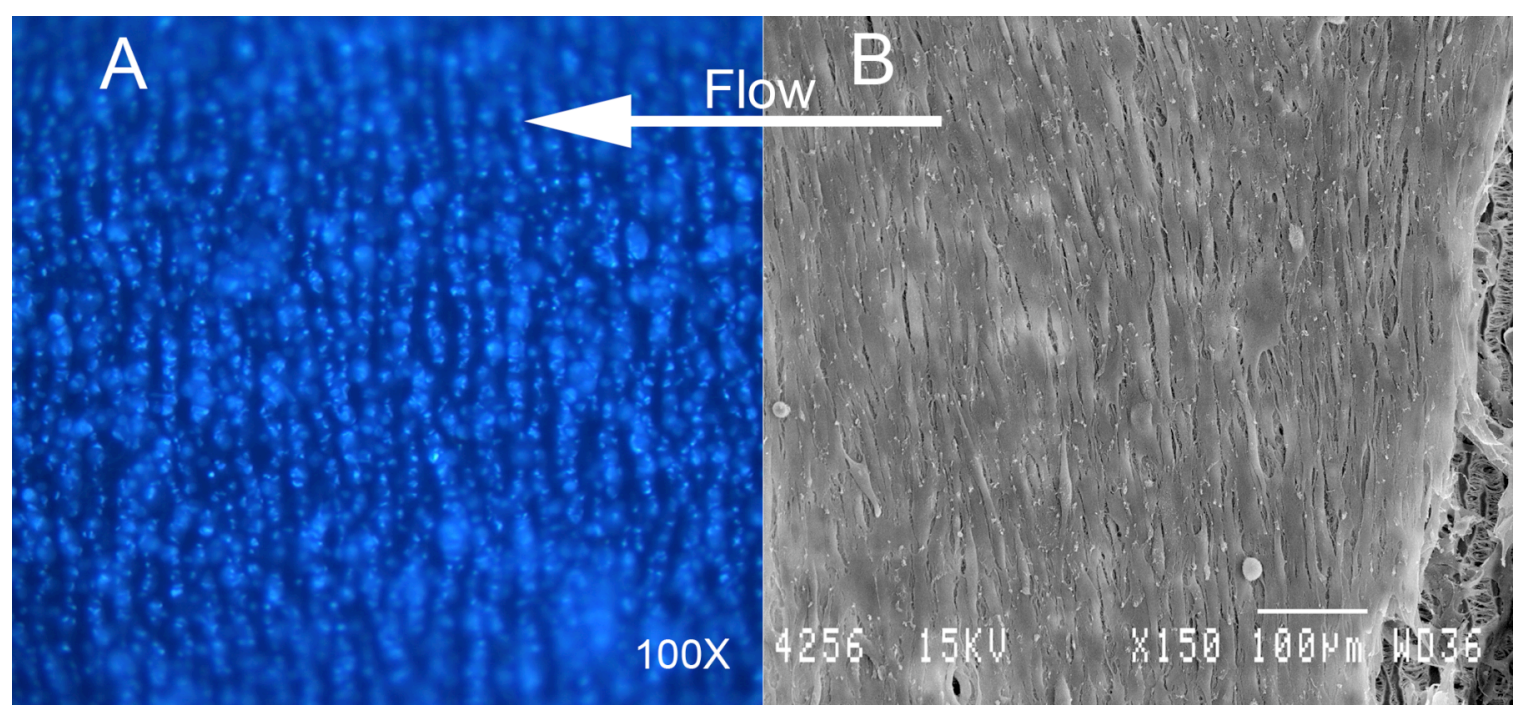

Figure 2.26 BBI(A) and SEM(B) images of ePTFE graft sodded with HMVECs. K. Cardinal, unpublished data.

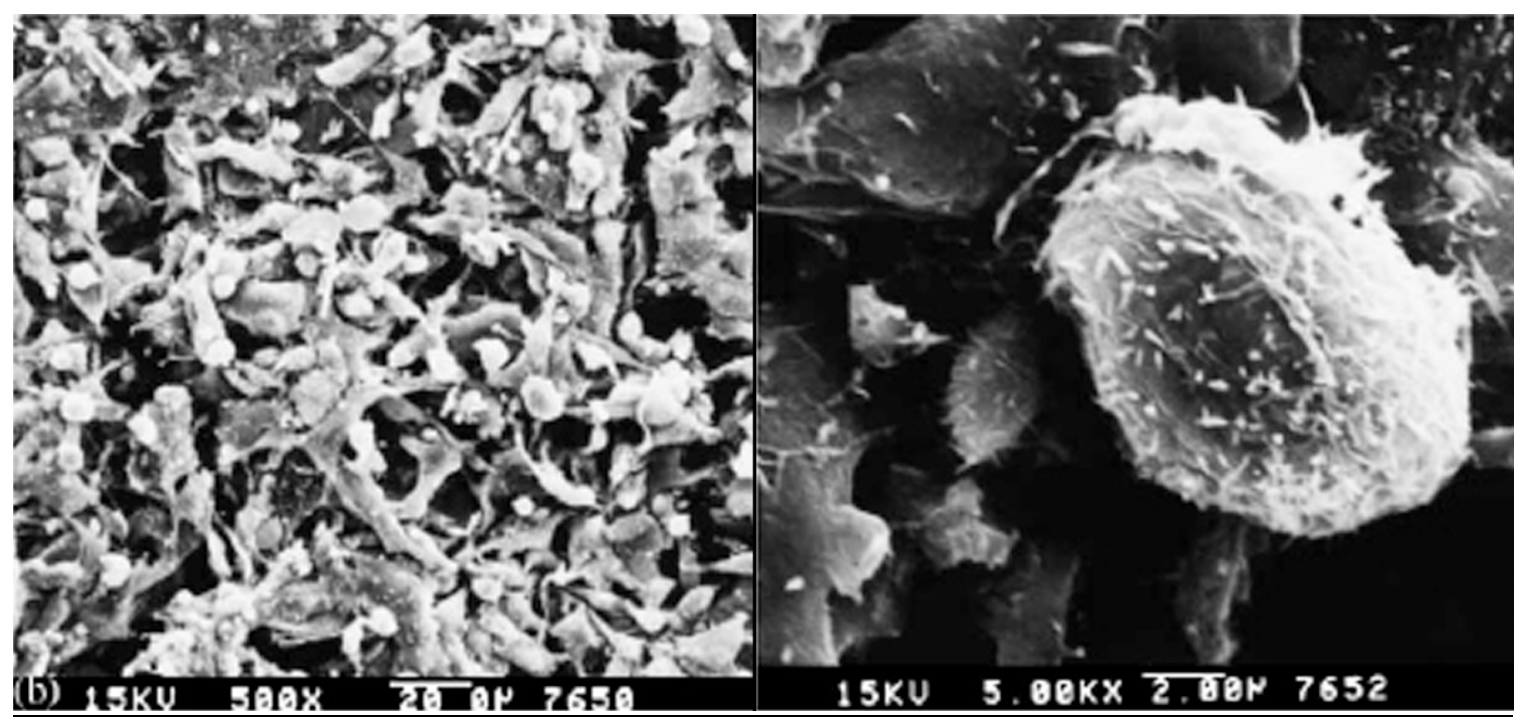

Figure 2.27 SEM images of HUVECs cultivated in a perfusion bioreactor system on a polyurethane graft [31]. (Permission to reprint figure granted by Elsevier publications. See Appendix D)

In addition to the possibility that the appearance of the grafts grown under the conditions described above was due to the cell type used, this result may also have been partially due to the qualities of the scaffold material. As is characteristic of ePTFE, there is little potential for radial distension as a result of the luminal pressure fluctuations. This may have prevented the cells from being exposed to the strain that is the ultimate goal of 
pulsatile flow. Although this strain has been shown to primarily affect the growth of SMCs, it has also been found to improve EC growth to some degree $[41,58,62,68]$.

While the lack of compliance may have limited the beneficial effects of pulsation, it may have also amplified its potential for negatively affecting construct growth. Due to the lack of scaffold compliance, the cyclic increases in luminal pressure may not have been relieved, resulting in compression of the cells against the scaffold. This possibility may explain both the poor cell morphology as well as the observation that the cells appeared to be lodged further in the internodal spaces than was expected. Currently, there are efforts to develop a scaffold for use in the BVM bioreactor system that will allow for the compliance necessary to expose sodded cells to the cyclic strain required to produce an ideal construct. 


\section{CHAPTER 3: ESTABLISHING AND CONTROLLING WALL SHEAR STRESS}

\section{INTRODUCTION}

As described in Chapter 1, wall shear stress experienced by the endothelial cells of blood vessels can have a profound effect on nearly every aspect of the vessel: cell phenotype, cell orientation, and even development and progression of certain diseases and conditions $[37,42,45,48,74,76,86]$. Before presenting the methods and results of this work, it is necessary to discuss some basic principles of shear stress in fluid flow, the physiologic importance of shear stress, the level of shear required to produce desired effects, the methods by which other groups have implemented shear stress in their systems, and the concept behind developing the desired shear stress in our model.

\section{Physics of Shear Stress in a Tube}

In the case of fluid flow, viscous or wall shear stress is the tangential force experienced by a solid boundary when the fluid flows past it. The properties of the fluid, the geometry and properties of the boundary surface and the characteristics of the flow itself can affect the wall shear stress experienced at the boundary $[17,82]$. Due to the fact that the scaffold material and geometry are essentially fixed in this study, these variables will be considered constant and wall shear stress will be addressed by considering only the fluid's viscosity and flow rate. Although there are many other possible factors to consider such as the pressure gradient across the diameter of the tube, the resistant properties of the tube material and varied geometries of the tube, this is beyond the scope of this work. 
Dynamic viscosity is the force per unit area required to move two horizontal planes past one another when the two planes are separated by constant distance [19]. In the case of the BVM, the two horizontal planes are the wall of the graft and the plane created by the perfusate flow. In this case, the calculated dynamic viscosity will be considered to be the same over the entire inner circumference of the graft surface. The international system (SI) unit of dynamic viscosity is the Pascal-second $(\mathrm{Pa} \bullet \mathrm{s})$ while the centimeter-gram-second (CGS) unit is the Poise (P) [17]. For purposes of this study, viscosity will be referred to in centipoise (cP), which is equal to $0.001 \mathrm{~Pa} \bullet \mathrm{s}$ [2]. The SI unit for shear stress is the Pascal (Pa), but for purposes of this work, shear stress will be referred to in dyne $/ \mathrm{cm}^{2}$, or $0.1 \mathrm{~Pa}[2]$.

\section{Effects of Shear Stress on Blood Vessel Physiology}

Proper phenotypic expression and alignment of vascular cells is significantly dictated by the shear forces experienced by the cells and therefore, the characteristics of flow within the vessel $[37,42,45,48,74,76,86]$. Shear stress aids in regulating phenotype by altering gene expression, which results in changes in the release of growth factors and adhesion molecules [45]. This can affect the cells on an individual, morphologic level as well as on the tissue level with observed changes in cell organization and grouping $[18,37,45]$.

Lee et al investigated the effects of shear stress on cell and stress fiber orientation in smooth muscle cells (SMCs) isolated from the coronary arteries of dogs [42]. They also aimed to determine the magnitude of shear and time required to illicit cellular responses in order to gain an understanding of the mechanism that drives this response 
[42]. By culturing the isolated cells in a shear-controlled environment, they found that increased shear stress influences the alignment of SMCs and, contrary to studies on the alignment of ECs, SMCs were found to arrange themselves perpendicular to flow [42]. This alignment was found to be dependent on stress magnitude as well as exposure time [42]. While cultures grown for $0-48$ hours under static or $1 \mathrm{dyne} / \mathrm{cm}^{2}$ conditions did not show signs of organized alignment, shear stresses between 10-20 dyne $/ \mathrm{cm}^{2}$ induced perpendicular orientation with the greatest degree of alignment resulting from exposure to the higher end of this range [42]. Additionally, the degree of alignment was found to be time-sensitive as orientation became perpendicular to flow more quickly (within 4 hours) at $20 \mathrm{dyne} / \mathrm{cm}^{2}$ and more extensive with longer exposure times, with the greatest alignment occurring after 48 hours of exposure [42]. Although a specific mechanism was not identified that directly controls the SMCs response to the shear stress, fluorescent staining of the cultures grown under increased shear revealed increased levels of perpendicular alignment of F-actin microfilaments compared to those grown in static conditions [42].

In a study performed by Malek et al, confluent BAEC monolayers were cultivated in a 20 dyne $/ \mathrm{cm}^{2}$ shear stress environment for 24 hours and then analyzed for morphology, orientation, presence of actin fibers, $\beta$-actin mRNA levels, and intracellular enzyme activity [45]. Microscopic visualization indicated a change from the "cobblestone" appearance of static EC cultures to a uniform alignment in the direction of flow when grown in increased shear conditions [45]. Similarly, actin fiber staining showed that the actin fibers that were radially arranged around the individual cells in static cultures formed "cables" directed parallel to flow [45]. This morphologic 
observation was further supported by the detection of increased levels of intracellular $\beta$ actin mRNA [45]. Intracellular calcium levels and tyrosine kinase (TK) activity were also found to affect morphologic changes [45]. When static cultures of BAECs were treated with calcium chelators such as BAPTA-AM, the shape of these cells was altered [45]. Additionally, when cells grown in increased shear were treated with herbimycin A, an inhibitor of TK, the morphologic changes and actin fiber formation were not observed [45]. The specific mechanisms by which calcium and TK act were not determined.

It has been demonstrated that endothelial cells (ECs) align parallel to the direction of flow when the perfusion fluid generates shear forces of $6-10 \mathrm{dyne} / \mathrm{cm}^{2}$ [48]. The mechanism for this is not well understood, but within 25 minutes of exposure to increased shear forces lamellipodae and "stress fibers," that showed random orientation about cells in static cultures became organized into "cables" that were parallel to the direction of flow. Masuda et al did not state it, but because these stress fibers contain actin, orientation may be facilitated by the fibers pulling the cell body in the desired direction in response to sensed shear stresses. Although viscous stress mainly affects endothelial cells due to the direct contact, it has also been shown to affect the alignment of smooth muscle cells (SMCs) [42]. When exposed to stresses above $10 \mathrm{dyn} / \mathrm{cm}^{2}$, SMCs were observed to self-orient themselves perpendicular to the direction of fluid flow [42]. This finding is consistent with the observed SMC orientation induced by cyclic radial distension that results from pulsatile flow [42].

Along with its influence on individual cell orientation and phenotype, shear stress affects the physiology of the vessel as a whole, with the manifestation and progression of conditions such as coronary artery disease and restenosis $[37,46,74,83]$. Using the same 
cellular mechanisms, this effect has also been characterized as the endothelial layer's atheroprotective response in regions of higher shear stresses [48]. By observing different regions of the vasculature while considering the flow patterns typical to that region, investigators noted that regions where shear stress was laminar and constant, there was less of a tendency to develop atherosclerotic tissue, indicating that a protective mechanism had also been activated in the endothelial layer [45,76]. The opposite was also shown to be true in regions where shear stress was typically variable and turbulent, with a mean stress of less than $6 \mathrm{dyne} / \mathrm{cm}^{2}$, there was a higher instance of atherosclerotic plaque formation [76]. For purposes of this thesis, the observed effects that shear stresses have on vascular cells confirms the need to establish physiologic shear stress in our BVM system in order to create a native-like construct.

\section{Importance of and Minimum Shear Stress Required for Cell Alignment}

With it understood that shear stress is a significant factor in the proper development of vascular cells and blood vessels as a whole, it is necessary to discuss the degree of shear stress that must be imposed on our system to produce the best possible construct. Although no absolute minimum shear stress requirement could be determined from existing studies, there was a general consensus on the approximate range of stress necessary for EC and SMC alignment; from 6-10 dyne/ $\mathrm{cm}^{2}[18,25,28,42,45,48]$.

One study investigated the effects of shear on endothelial progenitor cells (EPCs) [86]. It was found that EPCs respond to stresses as low as $0.1-2.5$ dyne $/ \mathrm{cm}^{2}$ [86]. Video analysis showed that confluent layers of EPCs exhibited signs of alignment parallel to flow within 10 minutes of exposure to shear stresses $\sim 2$ dyne $/ \mathrm{cm}^{2}$ [48]. After one hour of 
exposure to the same stress level, $\sim 70 \%$ of the cells' lamellipodae (stress fibers) had become aligned downstream and parallel to flow and after 24 hours the cells themselves had oriented their long axes in a similar manner [48]. Furthermore, it was observed that the alignment of the cells and their lamellipodae became random when shear stresses were reduced to stasis [48]. Although EPCs are not directly relevant to this thesis, their response is notable as it reinforces the need for a proper shear environment and may provide a basis for future work in our lab.

Among the research reviewed for this thesis, there is conflicting data regarding the minimum shear stress required to produce an ideal, native-like blood vessel construct. There is a common range, however, of $6-10$ dyne $/ \mathrm{cm}^{2}[18,28,37,42,45,48,74,76,86]$. For purposes of this thesis, the target shear stress will be $6-8 \mathrm{dyne} / \mathrm{cm}^{2}$ as the low flow rate and diameter of the graft make achieving the higher side of this range $\left(9-10 \mathrm{dyne} / \mathrm{cm}^{2}\right)$ difficult without making the viscosity of the media abnormally high.

\section{Previously Used Methods for Implementing Wall Shear Stress (WSS) in TEVGs}

Among the many studies on the effects of shear stress on the alignment and organization of vascular cells, the most common method for introducing shear forces to cell cultures involves the use of a parallel-plate flow system $[18,42,48,86]$. Some work has been done using a perfusion system similar to ours however; the objective of most of these studies does not focus directly on the morphologic and physiologic activity of vascular cells but rather on the development of a viable TEVG [37,25,28].

Masuda et al designed a system that aimed to characterize the alignment and morphologic response of bovine carotid arterial endothelial cells with real-time analysis 
[48]. The ECs were plated onto glass substrates, which were placed in a flow chamber that was equipped for video microscopy [48]. A peristaltic pump was used to drive the flow of the growth media, which was amended with polyvinylpyrolidone or methylcellulose to increase the viscosity [48]. The resulting viscosity of the media was not specified, but it is assumed that the shear stress was primarily controlled by adjusting the flow rate and distance between the glass plates. In addition to the need for shear stress in order to induce alignment of ECs, the key finding from this study was that cultures should consist of confluent monolayers when applying shear stresses, as it was found that sparsely cultured ECs exposed to $\geq 6$ dyne $/ \mathrm{cm}^{2}$ did not align with flow even after 100 hours of exposure [48]. This finding is relevant to the current thesis work as it presents the need to use low shear conditions during the sodding period and allow a confluent monolayer of cells to become established on the scaffold prior to introducing increased shear stress to the system.

Using a system similar to that of the Masuda group, Davies et al examined the effects of laminar versus turbulent flow under increased shear conditions on EC growth [18]. Bovine aortic endothelial cell monolayers were grown on glass coverslips in a modified cone-plate viscometer [18]. Shear stress in their system was controlled by adjusting the media flow rate and the distance between the growth plates [18]. Consistent with other studies of this type, normal growth and proper alignment (parallel to fluid flow) were observed in cultures subjected to shear stresses $\sim 8$ dyne $/ \mathrm{cm}^{2}$. An important result of this work was the observation that along with suitable shear stress, laminar as opposed to turbulent flow is also a requirement for proper growth. Even with shear 
stresses up to $14 \mathrm{dyne} / \mathrm{cm}^{2}$, if the flow was turbulent, the cell orientation became random and there was observed cell retraction and loss from the initial monolayer [18].

Koller et al used a TEVG style perfusion bioreactor system to investigate the effects of shear stress and flow on the dilation of arterioles [37]. First-order, 10-40 $\mu \mathrm{m}$ diameter, arterioles were isolated from six-week old Wistar rats and placed directly in a perfusion chamber by attaching micropipettes to each end of the vessel [37]. Pressure in the system was held constant at $60 \mathrm{mmHg}$ [37]. Dextran (MW 77,800) was added to the media at concentrations of $0,2,4$ and $6 \%$ to produce viscosities of $0.9,1.35,2.23$ and 3.16 centipoise respectively [37]. The wall shear stress, which ranged from $0-470$ dyne $/ \mathrm{cm}^{2}$, was calculated using $\tau_{\text {wall }}=4 \mu \mathrm{Q} / \pi \mathrm{r}^{3}$ [37]. The results presented by Koller et al indicated that arterioles dilate in response to increased shear forces, a finding that is consistent with native vessel response [37].

Guidicandrea et al presented a study that aimed to develop what they termed an "Ideal Flow Circuit" which was used to characterize the response of endothelial cells that were seeded onto grafts. The perfusion system they developed was quite complex as it had analysis probes that provided real-time feedback on the $\mathrm{pH}, \mathrm{pO} 2$ and $\mathrm{pCO} 2$ levels of the circulating media [25]. These levels were automatically adjusted by an oxygenator, mounted inline, with temperature control via a heat exchanger [25]. A pressure control system monitored and adjusted pulsation in the system by opening and closing a set of solenoid valves mounted inline [25]. Investigators made it clear that shear stress was essential in the development of their "ideal" system and relied on the addition of Dextran (MW 77,000) to increase the viscosity of their media. This allowed for the establishment of physiologic shear stresses of $\sim 7.6$ dyne $/ \mathrm{cm}^{2}$ with flow rates around $212 \mathrm{ml} / \mathrm{min}$ [25]. 
The study did not present any direct results regarding cell alignment or morphology, but they indicated that this shear stress improved cell retention on the graft and that the ECs present were more "normal" than those seen under lower shear stresses [25]. They also concluded that their system accurately simulates native conditions and could be used to study the behavior of ECs under varying conditions [25].

\section{Wall Shear Stress in the BVM System}

Without changing the geometry or composition of the scaffold, the fundamental methods for increasing and controlling wall shear stress in our BVM will involve adjusting flow rate and/or the viscosity of the fluid itself. Due to limitations of the current BVM system, appropriate wall shear stress cannot be achieved by simply increasing the flow rate, as the viscosity of the media is near that of water. Therefore, the composition of the media will be manipulated to control the shear stress sensed by the cells. The use of Dextran and Methylcellulose as media additives that increase the viscosity and thus wall shear stress has been shown to be suitable for use in vascular cell and TEVG cultivation $[25,37,48]$. Although it has not been used extensively for viscosity control in TEVG cultivation, Methylcellulose has been shown to have no adverse effects on endothelial cell cultures [19].

As with the development of pulsatile flow in our system, the implementation of shear stress was approached with the objective of achieving physiologic wall shear forces without making any major changes to the fundamental design, setup and operation of the BVM system. Due to the flow parameters established in the original system, there was limited flexibility in adjusting the luminal flow rate, as cell adherence to the ePTFE 
scaffold is sensitive. The original system's flow rate of $\sim 15 \mathrm{ml} / \mathrm{min}$ is relatively low when compared to other TEVG bioreactor setups such as those described above [12]. Thus, the approach taken here was to rely mainly on altering the viscosity of the perfusion fluid to increase shear.

The initial thought when approaching this objective was to simply add a thickening agent to the media in a concentration that would provide the desired viscosity and corresponding shear stress. Before attempting to evaluate the effects of shear stress in the BVM system, the first step was to determine whether or not these additives would be capable of adjusting viscosity to an appropriate value for use in our system.

\section{MATERIALS AND METHODS}

Determination of Necessary Viscosity

Based on the parameters set forth by the configuration of the original system, several assumptions were established when deriving an equation to calculate the wall shear stress seen at the inner wall of the BVM construct. For purposes of this work, luminal flow was assumed to be fully developed, pressure driven, steady and laminar. This does not take into account the presence of pulsation and how it can alter shear stresses in a tube, a factor that is beyond the scope of this thesis. In addition to the assumed flow characteristics, it was assumed that the construct scaffold is a perfectly circular tube. It was realized that the scaffold is made of a pliable material and may not maintain a perfectly circular profile, but for the purposes of these calculations this assumption was maintained. With these assumptions accepted, the following equation was established. 


$$
\tau_{\mathrm{wall}}=32 \mu \mathrm{Q} / \pi \mathrm{D}^{3}
$$

Where: $\tau_{\text {wall }}$ is the calculated wall shear stress ( $\mathrm{N} / \mathrm{m}^{2}$ or Pascal), $\mu$ is the viscosity of the perfusion fluid $\left(\mathrm{N} \bullet \mathrm{s} / \mathrm{m}^{2}\right), \mathrm{Q}$ is the volumetric flow rate $\left(\mathrm{m}^{3} / \mathrm{s}\right)$, and $\mathrm{D}$ is the tubing diameter of the construct graft $(\mathrm{m})$. This equation was used to calculate the minimum viscosity needed to achieve the desired minimum shear stress of 6 dyne $/ \mathrm{cm}^{2}$ or 0.6 Pascals. Additionally, it was used to determine the shear stress resulting from flowing media with different concentrations of dextran through the BVM construct.

\section{$\underline{\text { Media Formulation }}$}

\section{Methylcellulose}

Based on the literature provided by Sigma-Aldrich, methylcellulose (MC, SigmaAldrich $\mathrm{p} / \mathrm{n}$ M0555) had the potential to achieve the necessary viscosity in very low concentrations, as it is a rather large polymer. In order to prepare the growth media with added MC, the manufacturer's preparation protocol was followed. This procedure indicated that solubilization should be carried out using water as the solvent. However, for our purposes, it was necessary to use complete growth media in the place of water to maintain the proper concentrations of the other components. For initial tests 2, 4, 6, and $8 \%(\mathrm{~g} / \mathrm{ml})$ solutions were prepared. About one-third of the needed volume of media was agitated and heated to $80^{\circ} \mathrm{C}$. Once at temperature, the powdered methylcellulose was added and allowed to stir until thoroughly dispersed. To completely solubilize the solution, cold media was added to bring the solution to final volume. The solution was cooled while agitating for $\sim 45$ minutes. The solution was then filter sterilized. 


\section{Dextran}

Powdered Dextran from Leuconostoc mesenteroides was obtained from SigmaAldrich (Sigma-Aldrich p/n D4751). Based on previous experience with this polymer, it was known that the powder form could be added directly to the complete media, and with gentle heating and agitation, solubilization would occur. As with methylcellulose, 2, 4, 6, and $8 \%(\mathrm{~g} / \mathrm{ml})$ solutions of dextran were prepared for initial tests. Follow up tests included concentrations up to $30 \%$ dextran in complete growth media. The appropriate amount of powdered dextran was added to the corresponding volume of complete growth media in $50 \mathrm{ml}$ conical tubes at room temperature. The tubes were then placed in a $37^{\circ} \mathrm{C}$ water bath for $\sim 5$ minutes, and then agitated using a vortex until the powder was completely dissolved. For higher concentrations, additional heated time was required. These solutions were then filter sterilized. A higher MW dextran from Sigma Aldrich (P/N D4876) was later tested in the same manner to determine if desired viscosities could be achieved at lower polymer concentrations.

\section{Viscosity Testing}

\section{$\underline{\text { Dextran }}$}

The viscosity of the growth media was determined using a Vilastic-3 viscometer available from Vilastic Scientific Inc. This device is designed specifically for use with biologic fluids and determines viscosity and elasticity based on the rate at which the fluid oscillates in a cylindrical tube. The Vilastic-3 comes calibrated from the manufacturer but if necessary, calibration is performed using water as a baseline fluid. Based on its 
makeup and lack of any insoluble suspensions, it was assumed that the growth media was a Newtonian fluid.

Operation of the Vilastic-3 was very straightforward. Software test parameters were set based on manufacturer suggestions for Newtonian fluids in the desired viscosity range. These parameters included: oscillation frequency, integration time, low and high drive which were set to $2 \mathrm{~Hz}, 5$ seconds, 10 and 30 respectively. The low and high drive levels are unit less parameters that affect the amplification of the shear and strain rates exhibited by the unit during measurement. With parameters set, the fluid to be tested was drawn into the sample chamber at room temperature using a standard syringe. Once the sample was in position, the chamber was sealed and the test run was then initiated through the software interface (see Figure 3.1). The Vilastic software output the shear rate, shear strain and the shear stress seen in the sample tube as well as the viscosity and elasticity of the fluid.

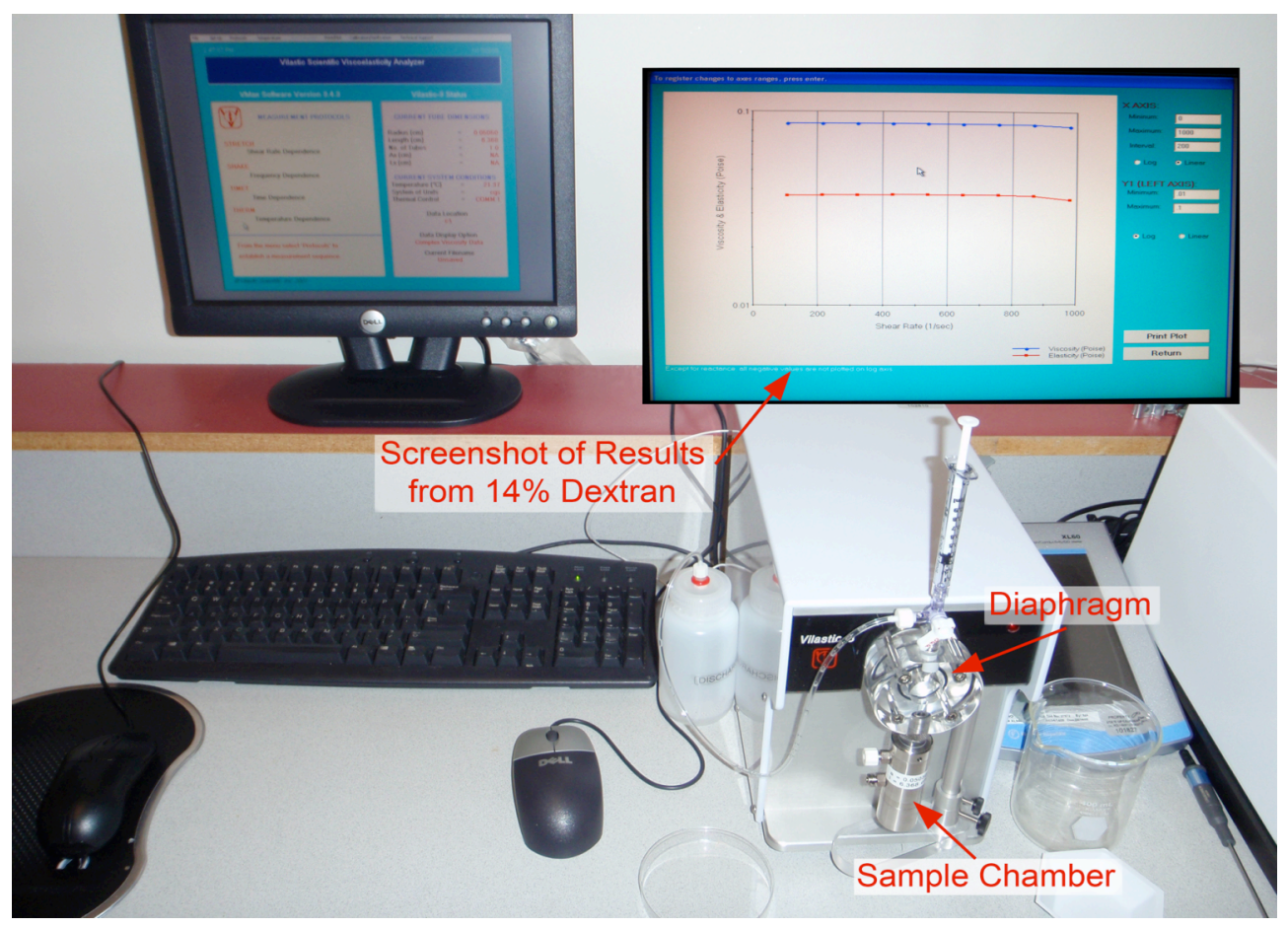

Figure 3.1 Vilastic-3 Viscometer system. 
The standard growth media containing $0 \%$ dextran was tested to determine the shear stress of the original setup. Subsequent samples were tested to determine what concentration of dextran would be required to establish the desired shear stress in our system. These viscosities were then used to calculate the shear stresses that would be experienced by the BVM constructs.

\section{$\underline{\text { Static Culture Test }}$}

Solutions of growth media containing $0,10,14,20$ and $30 \%$ dextran were used to grow HUVECs in static culture well plates to determine if the added dextran would alter the growth of the cells. HUVECs were grown to confluence in a $75 \mathrm{ml} \mathrm{T-flask} \mathrm{at} \mathrm{which}$ point they were used to inoculate a set of 6-well plates. After adding $5 \mathrm{ml}$ of endothelial cell media (ECM) with endothelial growth supplement from Science Cell to each well, 1 $\mathrm{ml}$ containing $\sim 5 \times 10^{5}$ cells was used to inoculate each well. After $\sim 24$ hours to allow the cells to become adhered to the well surface, the EC media was replaced with complete bioreactor media with the dextran concentrations mentioned above. Each concentration was grown in duplicate for a total of 10 wells. The cultures were monitored daily for five days to assess any changes in viable cell density, morphology and overall culture health. Light microscopy was used to visualize and photograph the cultures in order to compare the effects of the increased dextran concentrations with those grown sans dextran. For purposes of this study, culture evaluation was done on a qualitative basis with confluence estimated based on approximated surface coverage.

\section{Cell Adhesion Tests}


The final step of this work involved cultivating the BVM constructs using the higher viscosity media to determine if cell adhesion could be maintained under the increased shear conditions. For initial tests, Human Umbilical Vein Endothelial Cells (HUVECs) were sodded onto an ePTFE scaffold per the Sodding Protocol (Appendix B). These constructs were allowed to grow under steady flow and low shear conditions for 3 days to allow the cells to become attached to the scaffold under previously established conditions.

To avoid cell loss that may occur from a sudden increase in shear, the introduction of increased shear was done in a stepwise, ramp-up manner. After 3 days of growth using media containing no dextran, the low shear bioreactor media on the variable groups was replaced with complete bioreactor media containing $8 \%$ dextran. The control group received a new batch of bioreactor media with $0 \%$ dextran. For the initial change to viscous media ( $8 \%$ dextran), the flow rate was reduced to $10 \mathrm{ml} / \mathrm{min}$ to produce a wall shear stress of $\sim 1.15 \mathrm{dyn} / \mathrm{cm}^{2}$ to allow the cells to adapt to the increase in shear levels. After 24 hours of growth under these conditions, the $8 \%$ media was replaced with a $14 \%$ dextran solution and the flow rate was again reduced to $10 \mathrm{ml} / \mathrm{min}$ to avoid shocking the cells with a sudden increase in shear. The $14 \%$ dextran concentration was chosen as it will produce the desired minimum shear stress of $\sim 6.4 \mathrm{dyn} / \mathrm{cm}^{2}$. To achieve this, the flow rate was increased to $\sim 28 \mathrm{ml} / \mathrm{min}$. The flow rate was increased in hourly increments of $\sim 1.5 \mathrm{ml} / \mathrm{min}$ under steady flow conditions to $19 \mathrm{ml} / \mathrm{min}$ where it was held over night. The flow rate was increased in the same manner the next morning to $28 \mathrm{ml} / \mathrm{min}$.

For comparative purposes, constructs were maintained in two groups that were run at different flow rates (Table 3.1). Group A experienced lower shear conditions 
while Group B contained constructs that were exposed to shear forces intended to induce cell alignment $\left(\geq 6 \mathrm{dyn} / \mathrm{cm}^{2}\right)$.

Table 3.1 Shear stress cell adhesion test condition summary.

\begin{tabular}{|c|c|c|c|c|}
\hline Group/Construct \# & $\begin{array}{c}\text { Final } \\
{[\text { Dextran }]} \\
(\%)\end{array}$ & $\begin{array}{c}\text { Final Flow } \\
\text { Rate } \\
(\mathrm{ml} / \mathrm{min})\end{array}$ & $\begin{array}{c}\text { Final Wall } \\
\text { Shear Stress } \\
\left(\mathrm{dyn} / \mathrm{cm}^{2}\right)\end{array}$ & $\begin{array}{c}\text { Time at Final } \\
\text { WSS (hours })\end{array}$ \\
\hline SA1 & 0 & 15 & 0.3592 & 24 \\
\hline SA2 & 14 & 15 & 3.4251 & 24 \\
\hline SA3 & 14 & 15 & 3.4251 & 24 \\
\hline SB1 & 0 & 28 & 0.6709 & 8 \\
\hline SB2 & 14 & 28 & 6.3982 & 8 \\
\hline SB3 & 14 & 28 & 6.3982 & 24 \\
\hline
\end{tabular}

After 24 hours of growth under these conditions, all setups in Group A were taken down and the grafts were placed in Histochoice for later analysis using the same procedure described in the cell adhesion test section of Chapter 2. The control construct (SB1) and one exposed to $14 \%$ dextran (SB2) in Group B were taken down after 8 hours of flow at $28 \mathrm{ml} / \mathrm{min}$. The remaining construct in Group B (SB3) was taken down after 24 hours of exposure to $28 \mathrm{ml} / \mathrm{min}$. These samples were later analyzed using SEM and BBI staining.

\section{$\underline{\text { RESULTS }}$}

Estimating Required Viscosity

Based on the flow rate of $15 \mathrm{ml} / \mathrm{min}$, the needed viscosity to achieve the desired minimum shear stress of $6 \mathrm{dyn} / \mathrm{cm}^{2}$ was approximately 15.2 centipoise or 0.0152 Pa-s. This would produce a shear stress of $\sim 6.04 \mathrm{dyn} / \mathrm{cm}^{2}$ in the BVM with a $4 \mathrm{~mm}$ graft. If a $3 \mathrm{~mm}$ graft was used, the shear stress would be $\sim 14.3 \mathrm{dyn} / \mathrm{cm}^{2}$.

Viscosity Testing on Vilastic-3 Viscometer 


\section{Methylcellulose}

Due to the fact that the preparation procedure for methylcellulose was quite rigorous with regard to the temperature required to solubilize the polymer, it was thought that certain components of the media such as proteins may be damaged, making it a less desirable option. Filtration of the prepared solutions was problematic as well, with higher concentrations taking up to 45 minutes for filtration of $\sim 40 \mathrm{ml}$. Additionally, some unidentified particulates remained on the filter membrane after all of the solution had passed through the filter. Due to these difficulties, methylcellulose was abandoned as a potential additive in our system.

\section{$\underline{\text { Dextran }}$}

In contrast to methylcellulose, media containing dextran proved to be especially simple to prepare. The only difficulty was with solutions of higher concentrations, which required additional heating and agitation times. Filtration time was longer than standard media due to the added viscosity, but did not exceed 10 minutes. Resulting viscosities, along with luminal shear stress in a $4 \mathrm{~mm}$ graft, are shown below in Table 3.2-Table 3.4, Figure 3.2 and Figure 3.3. The figures highlighted in green in Tables 3.2-3.4 indicate conditions that create the minimum shear stress of $6 \mathrm{dyn} / \mathrm{cm}^{2}$. The viscosities and resulting shears stresses for concentrations below $10 \%$ and flow rates below $16 \mathrm{ml} / \mathrm{min}$ were omitted from the data chart, as they did not produce the minimum shear stress required for use in this system. This finding prompted the decision to increase the flow rate. 
Table 3.2 Calculated shear stress in BVM with $4 \mathrm{~mm}$ graft at $16-24 \mathrm{ml} / \mathrm{min}$.

\begin{tabular}{|c|c|c|c|c|c|c|c|c|c|c|}
\hline $\begin{array}{c}\text { Flow Rate } \\
\text { (ml/min) }\end{array}$ & 16 & 17 & 18 & 19 & 20 & 21 & 22 & 23 & 24 \\
\hline$\%$ Dextran & \multicolumn{9}{|c|}{ Shear Stress $\left(\mathrm{dyn} / \mathrm{cm}^{2}\right)$} \\
\hline $10.0 \%$ & 2.2785 & 2.4209 & 2.5633 & 2.7057 & 2.8481 & 2.9905 & 3.1358 & 3.2725 & 3.4178 \\
\hline $12.0 \%$ & 3.0456 & 3.2359 & 3.4263 & 3.6166 & 3.8070 & 3.9973 & 4.1915 & 4.3742 & 4.5683 \\
\hline $14.0 \%$ & 3.6535 & 3.8818 & 4.1102 & 4.3385 & 4.5669 & 4.7952 & 5.0281 & 5.2473 & 5.4802 \\
\hline $16.0 \%$ & 3.7384 & 3.9721 & 4.2057 & 4.4394 & 4.6730 & 4.9067 & 5.1450 & 5.3693 & 5.6076 \\
\hline $18.0 \%$ & 3.8321 & 4.0716 & 4.3111 & 4.5507 & 4.7902 & 5.0297 & 5.2740 & 5.5039 & 5.7482 \\
\hline $20.0 \%$ & 4.0900 & 4.3456 & 4.6012 & 4.8568 & 5.1125 & 5.3681 & 5.6288 & 5.8742 & 6.1350 \\
\hline $22.0 \%$ & 4.1471 & 4.4063 & 4.6655 & 4.9247 & 5.1839 & 5.4431 & 5.7075 & 5.9563 & 6.2207 \\
\hline $24.0 \%$ & 4.3416 & 4.6129 & 4.8842 & 5.1556 & 5.4269 & 5.6983 & 5.9751 & 6.2356 & 6.5123 \\
\hline $26.0 \%$ & 5.1140 & 5.4336 & 5.7532 & 6.0729 & 6.3925 & 6.7121 & 7.0381 & 7.3450 & 7.6710 \\
\hline $28.0 \%$ & 5.5364 & 5.8824 & 6.2284 & 6.5744 & 6.9204 & 7.2665 & 7.6194 & 7.9516 & 8.3045 \\
\hline $30.0 \%$ & 6.0089 & 6.3844 & 6.7600 & 7.1355 & 7.5111 & 7.8866 & 8.2697 & 8.6302 & 9.0133 \\
\hline
\end{tabular}

Table 3.3 Calculated shear stress in BVM with $4 \mathrm{~mm}$ graft at $25-33 \mathrm{ml} / \mathrm{min}$.

\begin{tabular}{|c|c|c|c|c|c|c|c|c|c|c|}
\hline $\begin{array}{c}\text { Flow Rate } \\
\text { (ml/min) }\end{array}$ & 25 & 26 & 27 & 28 & 29 & 30 & 31 & 32 & 33 \\
\hline$\%$ Dextran & \multicolumn{9}{|c|}{ Shear Stress $\left(\mathrm{dyn} / \mathrm{cm}^{2}\right)$} \\
\hline $10.0 \%$ & 3.5630 & 3.6997 & 3.8450 & 3.9902 & 4.1269 & 4.2722 & 4.4174 & 4.5542 & 4.6994 \\
\hline $12.0 \%$ & 4.7625 & 4.9452 & 5.1394 & 5.3335 & 5.5163 & 5.7104 & 5.9046 & 6.0873 & 6.2815 \\
\hline $14.0 \%$ & 5.7131 & 5.9323 & 6.1652 & 6.3982 & 6.6174 & 6.8503 & 7.0832 & 7.3024 & 7.5353 \\
\hline $16.0 \%$ & 5.8460 & 6.0703 & 6.3086 & 6.5469 & 6.7712 & 7.0096 & 7.2479 & 7.4722 & 7.7105 \\
\hline $18.0 \%$ & 5.9925 & 6.2224 & 6.4667 & 6.7110 & 6.9409 & 7.1852 & 7.4295 & 7.6595 & 7.9038 \\
\hline $20.0 \%$ & 6.3957 & 6.6411 & 6.9018 & 7.1626 & 7.4080 & 7.6687 & 7.9294 & 8.1748 & 8.4356 \\
\hline $22.0 \%$ & 6.4850 & 6.7339 & 6.9982 & 7.2626 & 7.5114 & 7.7758 & 8.0402 & 8.2890 & 8.5534 \\
\hline $24.0 \%$ & 6.7891 & 7.0496 & 7.3264 & 7.6031 & 7.8636 & 8.1404 & 8.4172 & 8.6777 & 8.9544 \\
\hline $26.0 \%$ & 7.9970 & 8.3038 & 8.6298 & 8.9559 & 9.2627 & 9.5887 & 9.9147 & 10.2216 & 10.5476 \\
\hline $28.0 \%$ & 8.6575 & 8.9896 & 9.3426 & 9.6955 & 10.0277 & 10.3807 & 10.7336 & 11.0658 & 11.4187 \\
\hline $30.0 \%$ & 9.3963 & 9.7569 & 10.1399 & 10.5230 & 10.8835 & 11.2666 & 11.6497 & 12.0102 & 12.3933 \\
\hline
\end{tabular}

Table 3.4 Calculated shear stress in BVM with $4 \mathrm{~mm}$ graft at $34-40 \mathrm{ml} / \mathrm{min}$.

\begin{tabular}{|c|c|c|c|c|c|c|c|}
\hline $\begin{array}{c}\text { Flow Rate } \\
(\mathrm{ml} / \mathrm{min})\end{array}$ & 34 & 35 & 36 & 37 & 38 & 39 & 40 \\
\hline$\%$ Dextran & \multicolumn{7}{|c|}{ Shear Stress $\left(\mathrm{dyn} / \mathrm{cm}^{2}\right)$} \\
\hline $10.0 \%$ & 4.8447 & 4.9814 & 5.1266 & 5.2719 & 5.4086 & 5.5538 & 5.6965 \\
\hline $12.0 \%$ & 6.4756 & 6.6584 & 6.8525 & 7.0467 & 7.2294 & 7.4236 & 7.6143 \\
\hline $14.0 \%$ & 7.7682 & 7.9874 & 8.2203 & 8.4532 & 8.6725 & 8.9054 & 9.1342 \\
\hline $16.0 \%$ & 7.9488 & 8.1731 & 8.4115 & 8.6498 & 8.8741 & 9.1124 & 9.3465 \\
\hline $18.0 \%$ & 8.1481 & 8.3780 & 8.6223 & 8.8666 & 9.0965 & 9.3408 & 9.5808 \\
\hline $20.0 \%$ & 8.6963 & 8.9417 & 9.2024 & 9.4632 & 9.7086 & 9.9693 & 10.2254 \\
\hline $22.0 \%$ & 8.8178 & 9.0666 & 9.3310 & 9.5954 & 9.8442 & 10.1086 & 10.3683 \\
\hline $24.0 \%$ & 9.2312 & 9.4917 & 9.7685 & 10.0453 & 10.3058 & 10.5825 & 10.8544 \\
\hline $26.0 \%$ & 10.8736 & 11.1804 & 11.5065 & 11.8325 & 12.1393 & 12.4653 & 12.7856 \\
\hline $28.0 \%$ & 11.7717 & 12.1038 & 12.4568 & 12.8097 & 13.1419 & 13.4949 & 13.8416 \\
\hline $30.0 \%$ & 12.7763 & 13.1369 & 13.5199 & 13.9030 & 14.2635 & 14.6466 & 15.0229 \\
\hline
\end{tabular}




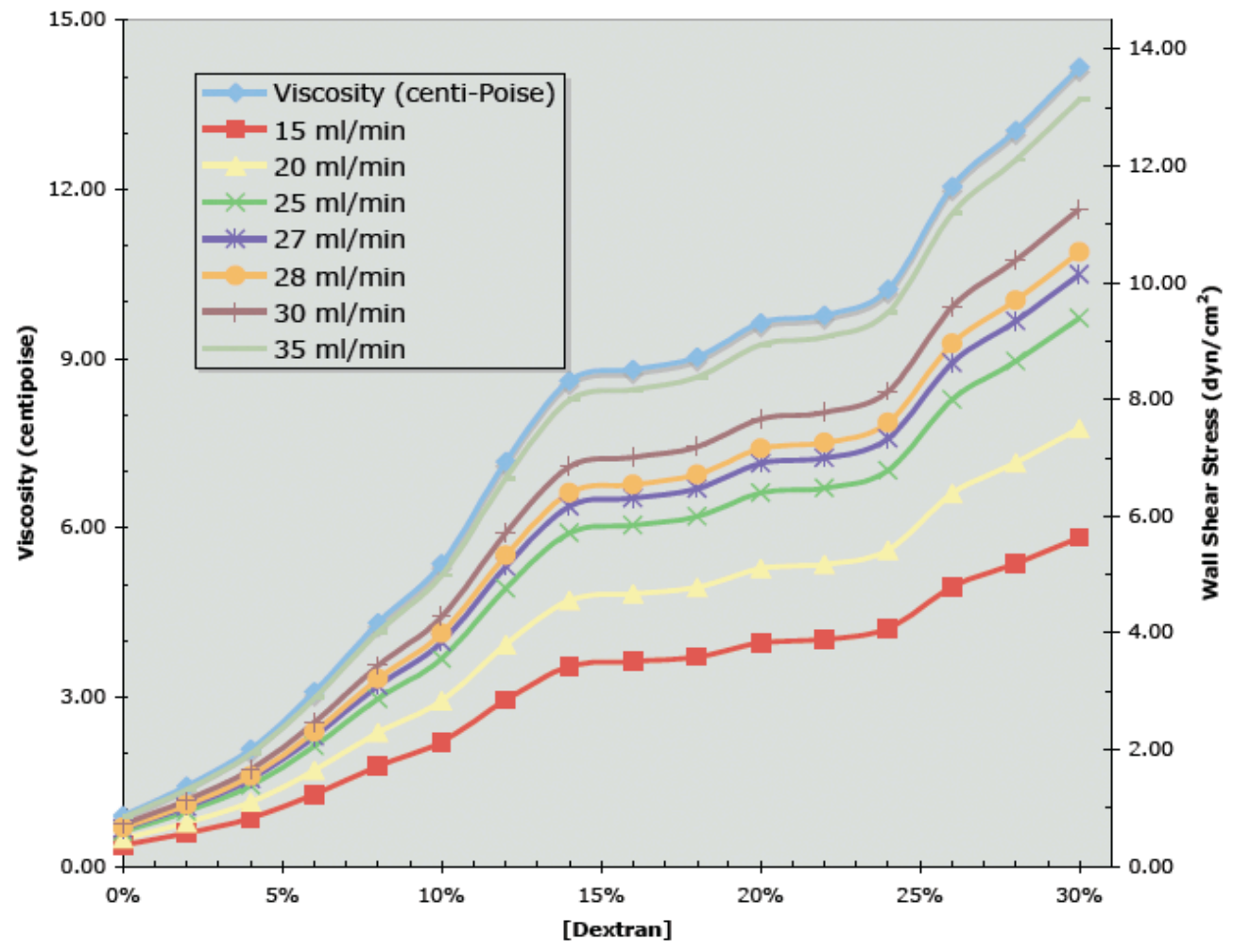

Figure 3.2 Viscosity and shear stress of increasing Dextran concentrations at different flow rates.

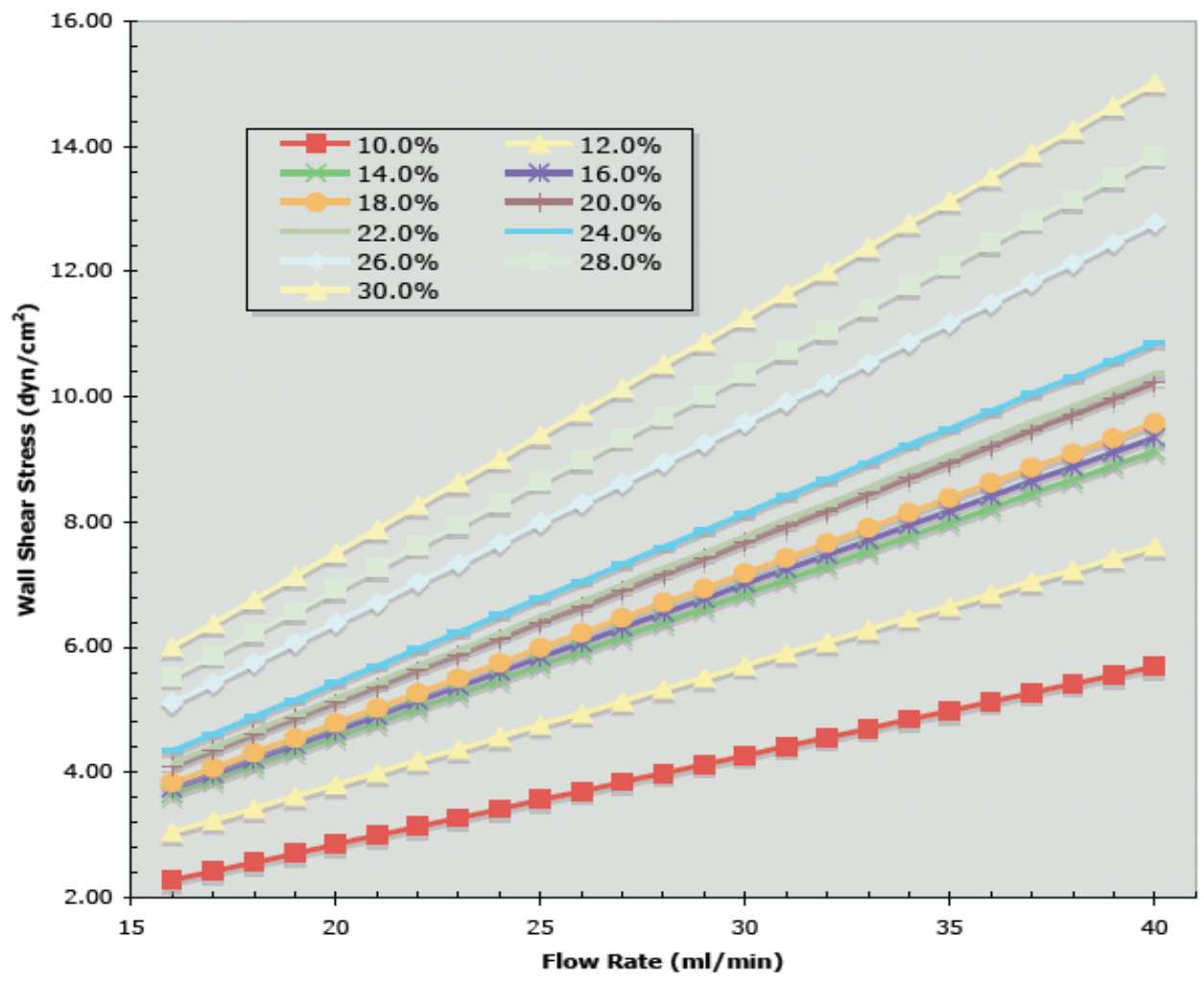

Figure 3.3 Shear stress with increasing flow rates of growth media with varied concentrations of Dextran. 


\section{Characterization of Media Viscosity}

As mentioned previously, it was assumed that complete growth media with and without added dextran was a Newtonian fluid and would not exhibit a change in viscosity with changing shear rates. However, to be certain that this was the case, the solutions were tested at different shear rates and were found, in fact, to have the properties of a Newtonian fluid (Figure 3.4).

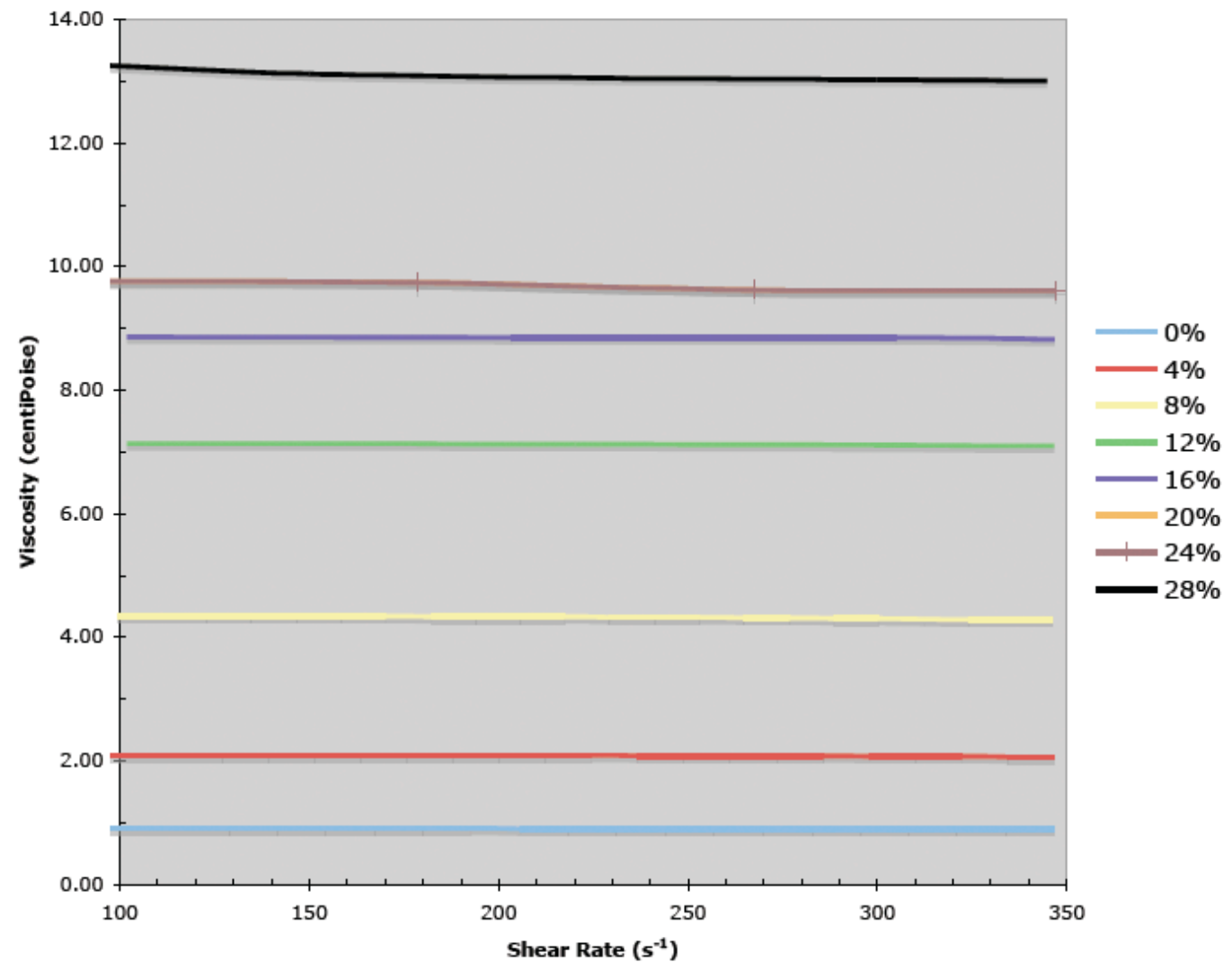

Figure 3.4 Viscosity of different Dextran concentration at varying shear rates to show Newtonian property of media. This data was extrapolated from the results of viscosity testing of each dextran concentration.

\section{Establishing Desired Wall Shear Stress}

As mentioned previously, one of the main objectives of this thesis work was to implement the desired conditions with as little disruption to the original system as 
possible. However, upon determining the viscosity of the various concentrations of dextran, it was realized that in order to achieve the desired minimum shear stress in the system of $6 \mathrm{dyn} / \mathrm{cm}^{2}$ with the current flow rate of $15 \mathrm{ml} / \mathrm{min}$, the media would have to be over 30\% dextran (Sigma $\mathrm{p} / \mathrm{n} 4751$ ). It was decided that this concentration was too high, as it may adversely affect cellular growth. Thus, a higher molecular weight dextran (Sigma $\mathrm{p} / \mathrm{n}$ D4876) was investigated with the idea that it could provide the desired viscosity at lower concentrations. However, it was found that the higher molecular weight dextran (D4876) actually produced a slightly lower viscosity at comparable concentration and thus was abandoned as an option (Table 3.5).

\section{Table 3.5 Viscosity of lower and higher molecular weight dextran in complete growth media.}

\begin{tabular}{|c|l|l|}
\hline & $\begin{array}{l}\text { Sigma D4751: MW 64E3- } \\
\text { 76E3 daltons }\end{array}$ & $\begin{array}{l}\text { Sigma D4876: MW 100E- } \\
\text { 200E3 daltons }\end{array}$ \\
\hline [Dextran] & \multicolumn{2}{|c|}{ Viscosity (cP) } \\
\hline $0 \%$ & 0.9027 & 0.9027 \\
\hline $2 \%$ & 1.4191 & 1.3616 \\
\hline $4 \%$ & 2.0840 & 1.7825 \\
\hline $6 \%$ & 3.1012 & 2.2672 \\
\hline $8 \%$ & 4.3217 & 3.4908 \\
\hline $10 \%$ & 5.3686 & 4.1910 \\
\hline
\end{tabular}

In order to achieve the minimum shear stress of $6 \mathrm{dyn} / \mathrm{cm}^{2}$, it was decided that the flow rate would have to be increased to avoid the need for excessively high dextran concentrations. In an effort to balance the need to use the lowest possible dextran concentration and flow rate that would provide desired shear stresses, it was decided that the system would be run at $27-28 \mathrm{ml} / \mathrm{min}$ with media consisting of $14 \%$ dextran $(\mathrm{g} / \mathrm{ml})$.

\section{Static Culture Tests}


After one day of growth in media containing endothelial cell growth supplement, the cells had become well adhered to the bottom surface of the wells with $\sim 95-100 \%$ confluence (Table 3.6 and Figure 3.5). After 24 hours (Day1) of growth in the bioreactor media with and without dextran, cultures grown in the solution containing 0,10 and $14 \%$ dextran all showed similar growth with an observed adhesion and extension of the cell bodies and their lamellipodae (Figure 3.6). The cultures grown in $20 \%$ dextran showed acceptable adhesion, however, the morphology showed a moderate level of lamellipodae retraction resulting in a more spherical appearance (Figure 3.6).

\section{Table 3.6 Estimated confluence of static cultures of HUVECs grown in bioreactor media amended with varied amounts of dextran.}

\begin{tabular}{|c|c|c|c|c|c|c|}
\hline $\begin{array}{c}\text { [Dextran] } \\
(\%)\end{array}$ & Day 0 & Day 1 & Day 2 & Day 3 & Day 4 & Day 5 \\
\cline { 2 - 7 } & \multicolumn{6}{|c|}{ Confluence/Adherence to Well Surface } \\
\hline 0 & 100 & 100 & 100 & 95 & 90 & 90 \\
\hline 10 & N/A & 95 & 95 & 90 & 85 & 85 \\
\hline 14 & N/A & 95 & 95 & 90 & 85 & 85 \\
\hline 20 & N/A & 90 & 85 & 80 & 75 & 65 \\
\hline 30 & N/A & 85 & 75 & 60 & 40 & 20 \\
\hline
\end{tabular}

The decrease in confluence relative to increasing dextran concentration as seen in Table 3.6, may have been due to cytotoxicity of higher dextran levels or a decrease in the gas permeability of the higher viscosity medias that contained higher dextran concentrations. The decrease in confluence relative to culture time (Days) was most likely due to nutrient depletion of the static media. This trend was consistent in cultures grown in 30\% dextran with an even more extensive degree of lamellipodae retraction and spherical cell morphology (Figure 3.6). The following days showed a similar progression with cultures grown in 0,10 and 14\% maintaining similar adhesion and cell extension. After 48 hours (Day 2) of growth, cultures containing 10 and 14\% dextran did show some cell loss, but not to any significant degree. However, the cultures grown in 20 and $30 \%$ dextran for 
two days began to exhibit extensive retraction and increased release from the well surface. After 72 hours (Day 3), the 0, 10 and 14\% cultures maintained comparable cell adhesion and morphology with a slight increase in cell loss. At this point, those grown in 20 and $30 \%$ continued to degrade at a more rapid rate. Particularly, the cultures containing 30\% dextran contained clumps of dead or released cells. This overall trend continued through Day 5 of culture growth with near complete culture loss in the wells containing 30\% dextran.

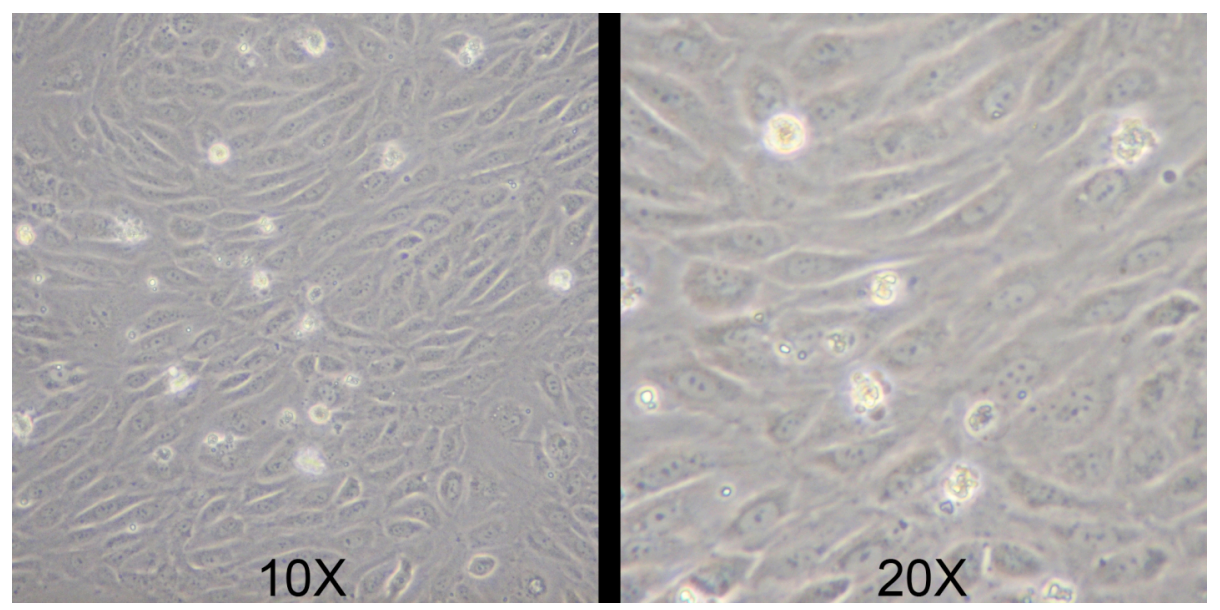

Figure 3.5 HUVECs static culture -Day 0. 

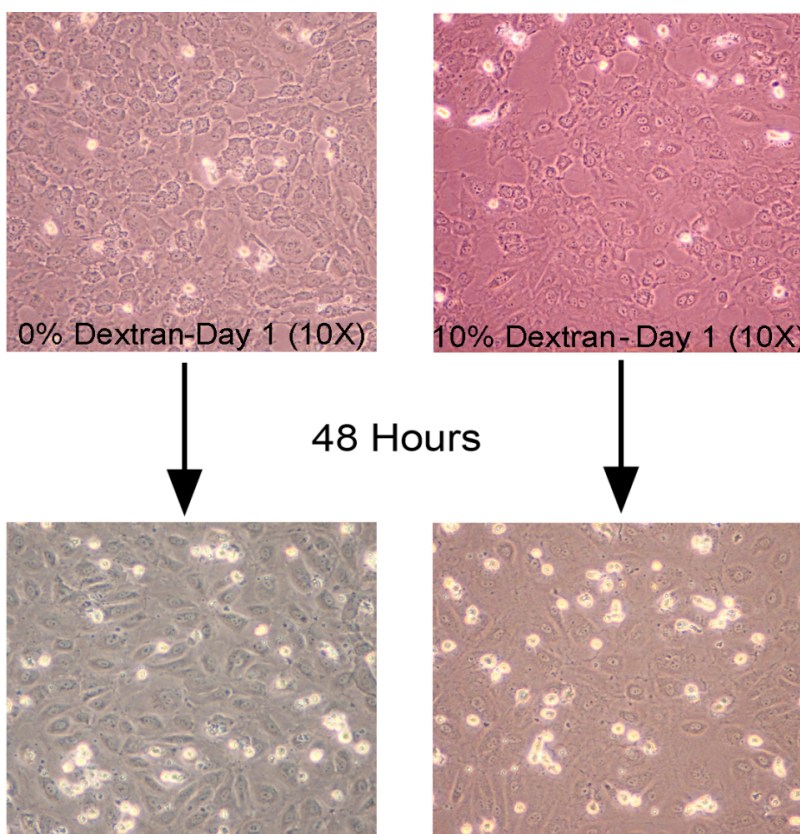

48 Hours

10\% Dextran-Day $1(10 x$

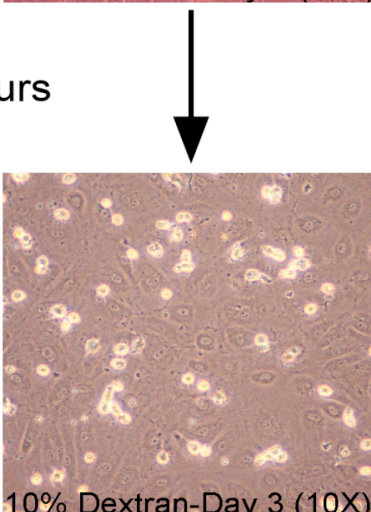

$0 \%$ Dextran-Day $3(10 X)$
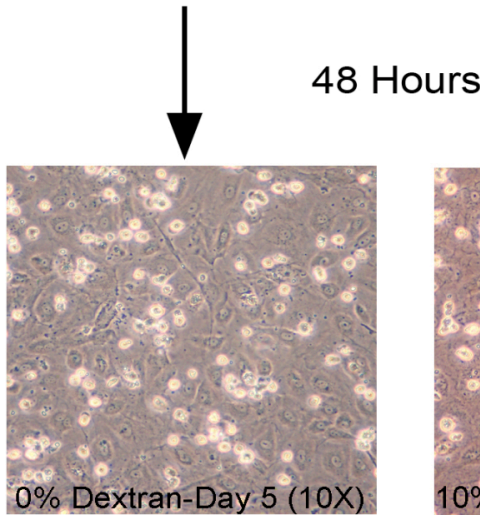

Dextran-Day $3(10 x)$
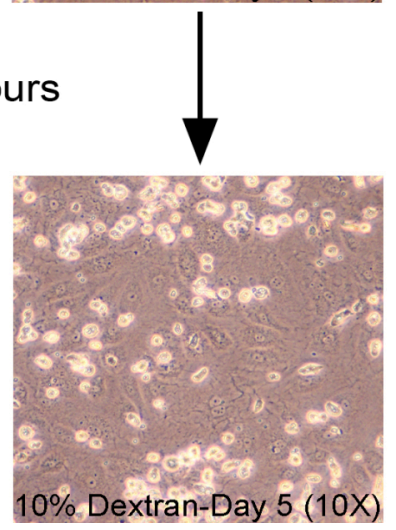

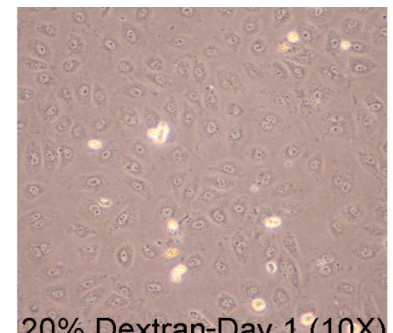

$14 \%$ Dextran-Day 1 (10X)

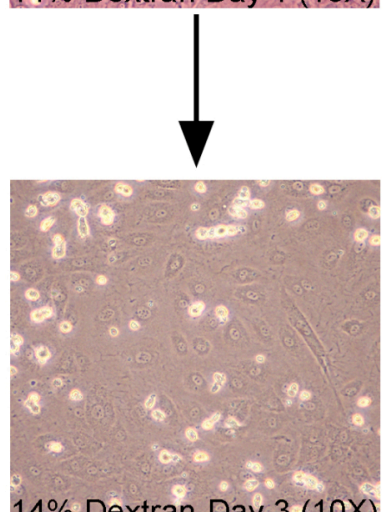

$14 \%$ Dextran-Day $3(10 x)$

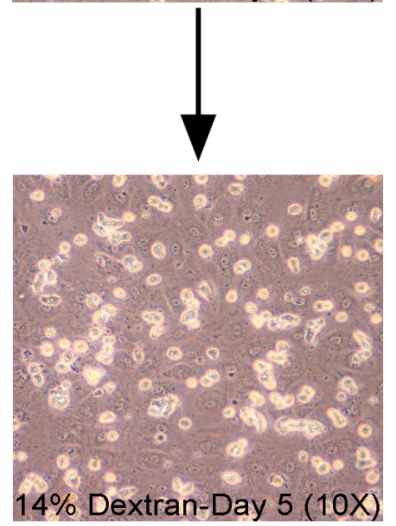

$20 \%$ Dextran-Day $1(10 x)$

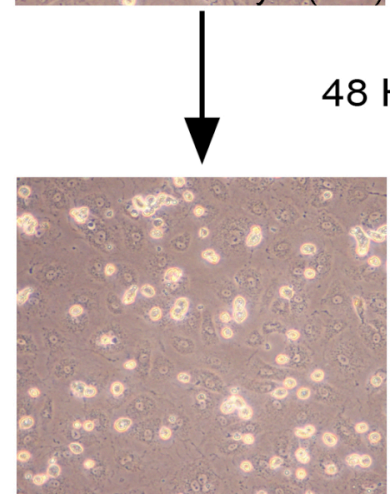

20\% Dextran-Day $3(10 x)$

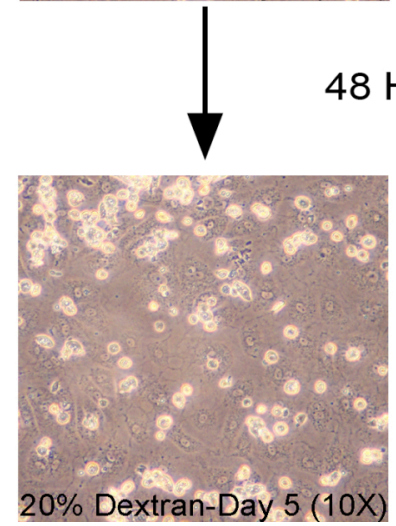

48 Hours

$30 \%$ Dextran-Day $3(10 x)$
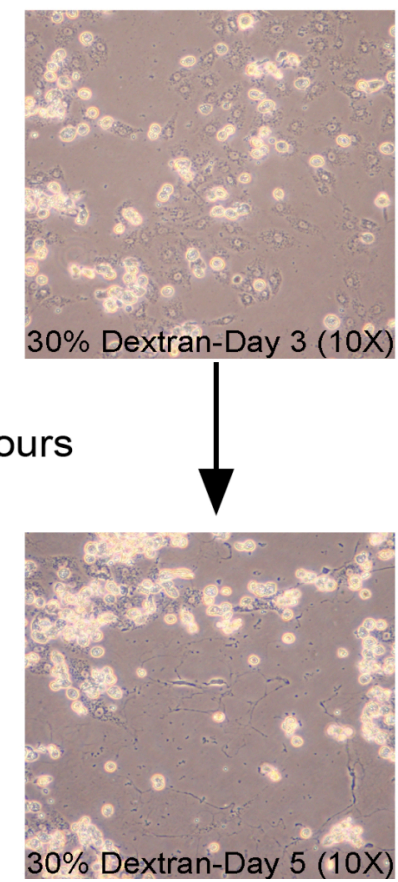

Figure 3.6 Cell morphology and culture appearance of static cultures of HUVECs grown in bioreactor media amended with varied amounts of dextran. Arrows indicate an elapsed time of 48 hours. 
From the results of this static culture growth test, it is concluded that the use of

dextran is not toxic to HUVECs as the culture maintained growth in media containing dextran for 5 days. Additionally, when used in concentrations up to $14 \%$, dextran does not affect the morphology and overall culture health to any significant degree. However, when used in static cultures at higher concentrations, there was an observed significant degree of cell loss, change in morphology and decrease in overall culture health. This is not to say that these concentrations are unsafe for culture use, however before they are implemented, further studies should be performed to investigate these effects in perfusion systems where growth conditions are more dynamic. Although it was not determined, the greater degree of cell loss in cultures containing higher concentrations of dextran (20 and $30 \%$ ) may be due to a decrease in gas exchange and nutrient transfer caused by the increased viscosity of these solutions.

\section{Cell Adhesion Tests}

The setup of the six BVM constructs proceeded without any major difficulties. As in the cell adhesion test in Chapter 2, each graft was sodded with approximately $7 \mathrm{x}$ $10^{6}$ cells resulting in a sodding density of $\sim 1.6 \times 10^{6}$ per $\mathrm{cm}^{2}$. When placing the grafts into the chambers, extra care was taken to avoid installing any of them under tension as was experienced in the pulsatile flow cell adhesion trials.

The initial 3-day growth period following sodding was carried out under steady flow conditions at $15 \mathrm{ml} / \mathrm{min}$ using standard media containing no dextran. The only complications found during this growth period were minor leaks in the pump tubing of constructs SA2 and SB2. The leaks were sealed and the tubing was moved on the pump 
head to avoid further cracking. After 72 hours of growth under these conditions, the media was changed to establish the test conditions outlined in Table 3.1. Each bioreactor system was then taken down at the appropriate time points without any adverse observations. Analysis with BBI staining and SEM indicated that the cells remained on all of the grafts following exposure to all levels of wall shear stress.

Analysis of the control construct, SA1, provided more expected results than the control construct in the Chapter 2 trials. BBI imaging indicated the presence of many nuclei that appeared to be on and in between the nodes of the ePTFE scaffold. SEM analysis confirmed this, as there was nearly complete filling of the internodal spaces and considerable coverage of the nodes themselves. There was also a considerable degree of cell spreading and/or matrix deposition as seen in the SEM image of Figure 3.7. The morphology was different than was expected as there was not a solid monolayer of cells, but as mentioned in Chapter 2, this may be in part due to the growth characteristics of HUVECs.

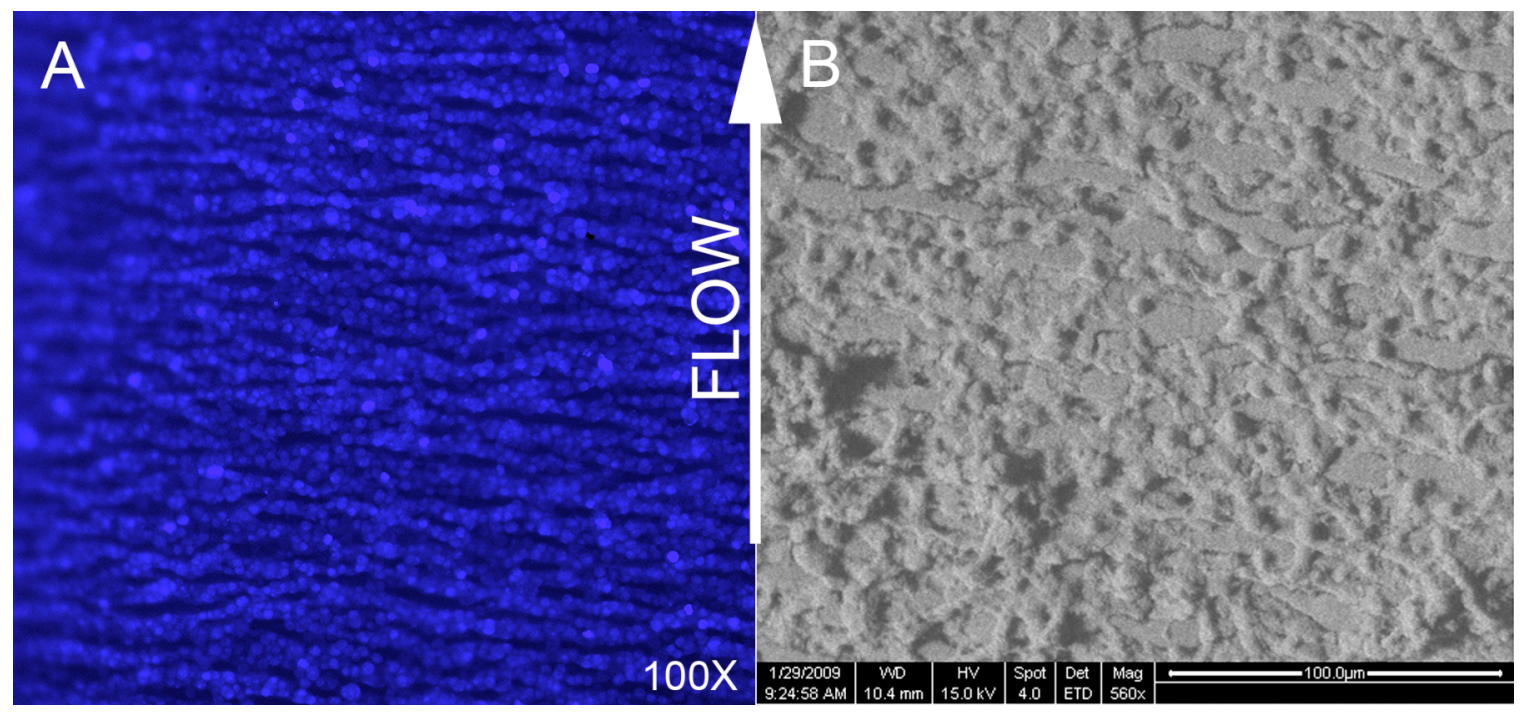

Figure 3.7 BBI(A) and SEM(B) imaging of construct SA1 (0\% Dextran, $15 \mathrm{ml} / \mathrm{min}$ $0.3592 \mathrm{dyn} / \mathrm{cm}^{2}$ for 24 hours). 
Imaging of the constructs exposed to mid levels of wall shear stress $(\sim 3.43$ dyn $/ \mathrm{cm}^{2}$ ) showed that the cells remained on the graft. As seen in Figure 3.8, the nuclei present on construct SA2 appeared to be somewhat larger than those in construct SA1. However visualization with SEM revealed that the surface cell density was not as extensive as the control indicating some cell loss caused by exposure to these shear stress levels. The cells present also showed an increased degree of clumping. The results of construct SA3 are not shown, as it was cultivated under identical conditions as SA2 and it was similar in appearance (Figure A.13 and Figure A.14).

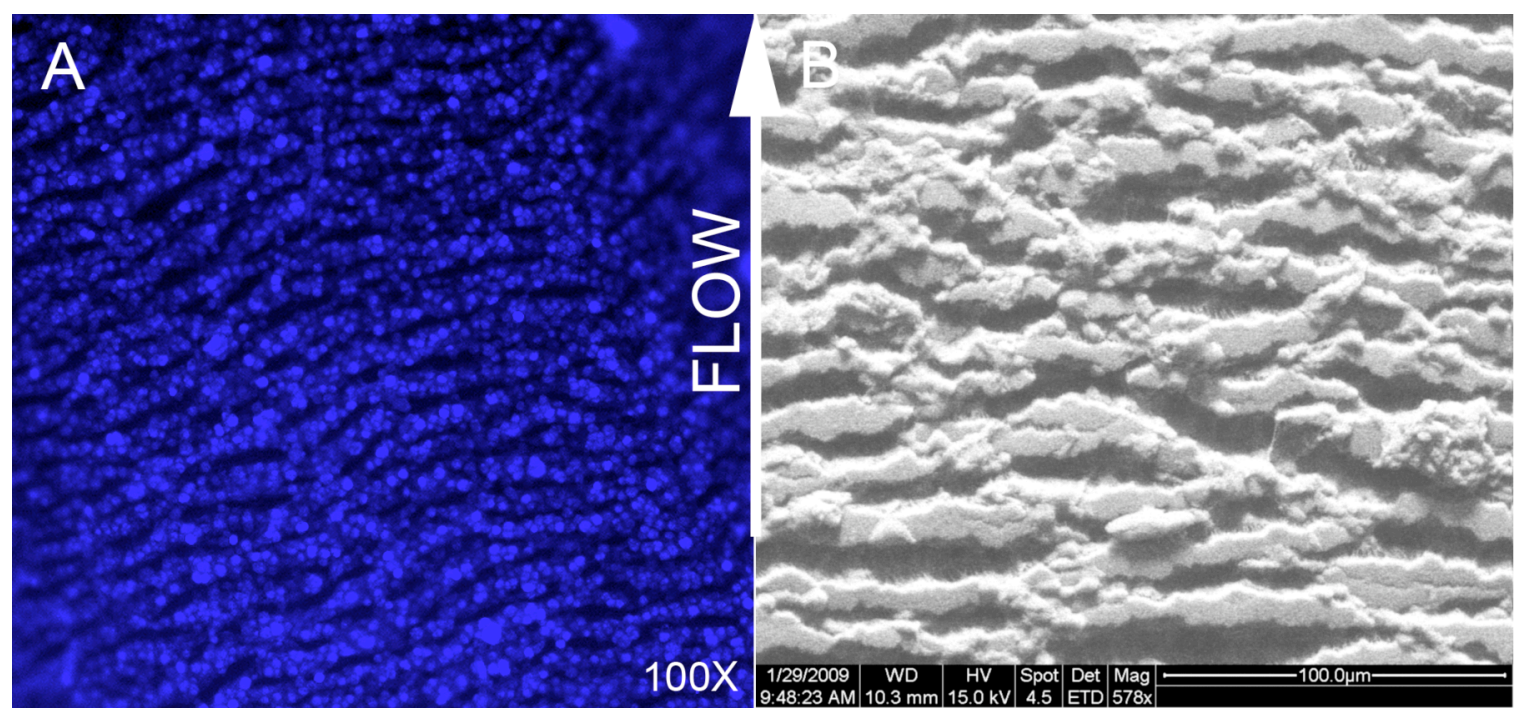

Figure 3.8 BBI(A) and SEM(B) imaging of construct SA2 (14\% Dextran, 15 $\mathrm{ml} / \mathrm{min}$, $3.4251 \mathrm{dyn} / \mathrm{cm}^{2}$ for 24 hours).

The SB1 construct was cultivated under an increased flow rate, but without any Dextran added to the media. The wall shear stress experienced by the cells in this graft was $\sim 0.671 \mathrm{dyn} / \mathrm{cm}^{2}$ for eight hours. The BBI image of this construct showed a similar number of cell nuclei present and although surface cell coverage was slightly more sparse than the lower flow control (SA1), it was higher than that of construct SA2. Note that the SEM image of SB1 in Figure 3.9 is taken at a higher magnification. 


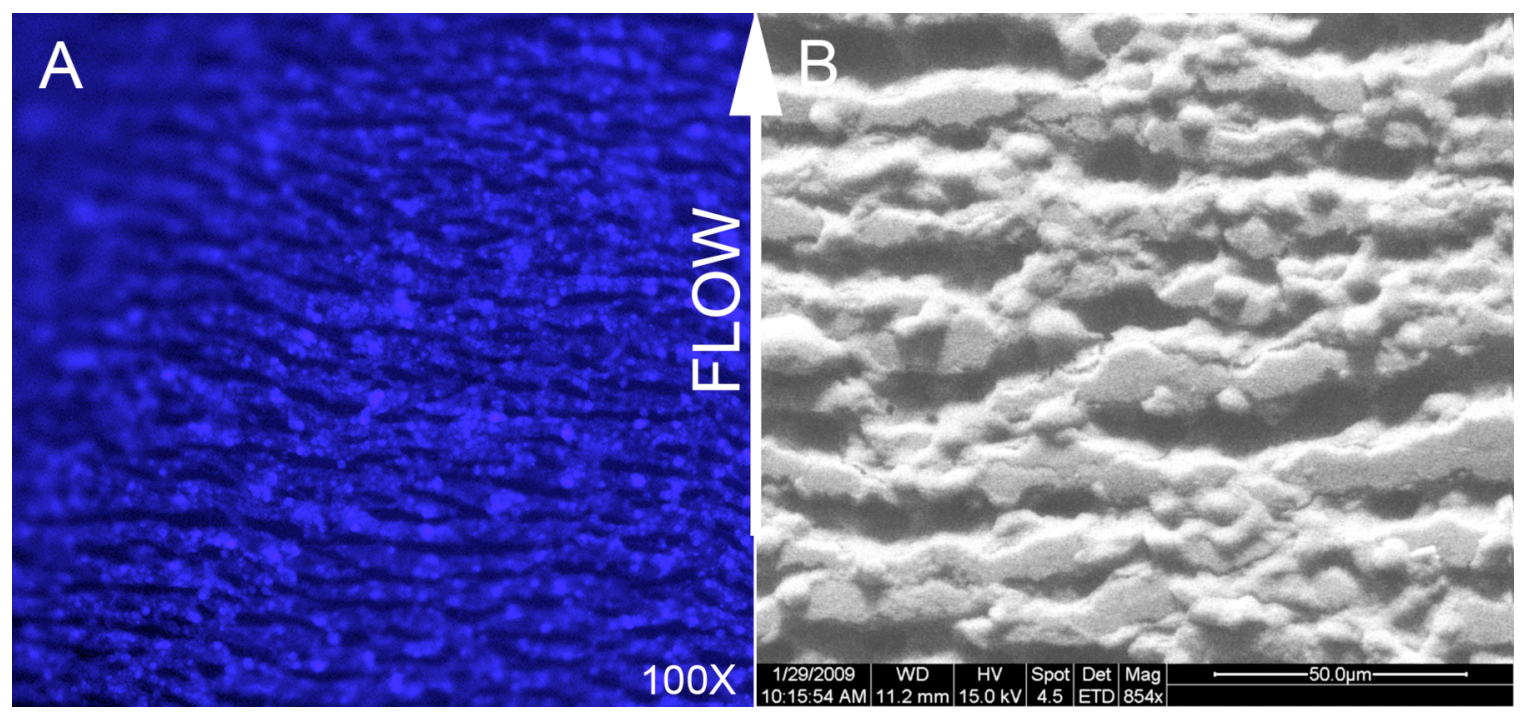

Figure 3.9 BBI(A) and SEM(B) imaging of construct SB1 (0\% Dextran, 28ml/min, $0.6709 \mathrm{dyn} / \mathrm{cm}^{2}$ for 8 hours).

Construct SB2 was exposed to $\sim 6.4 \mathrm{dyn} / \mathrm{cm} 2$ for 8 hours. Analysis of this construct confirmed the retention of the cells on the surface (Figure 3.10). There were many nuclei present on the nodes of the scaffold as shown in the BBI image. SEM imaging showed a moderate degree of filling of the internodal spaces and little cell extension and/or matrix deposition.

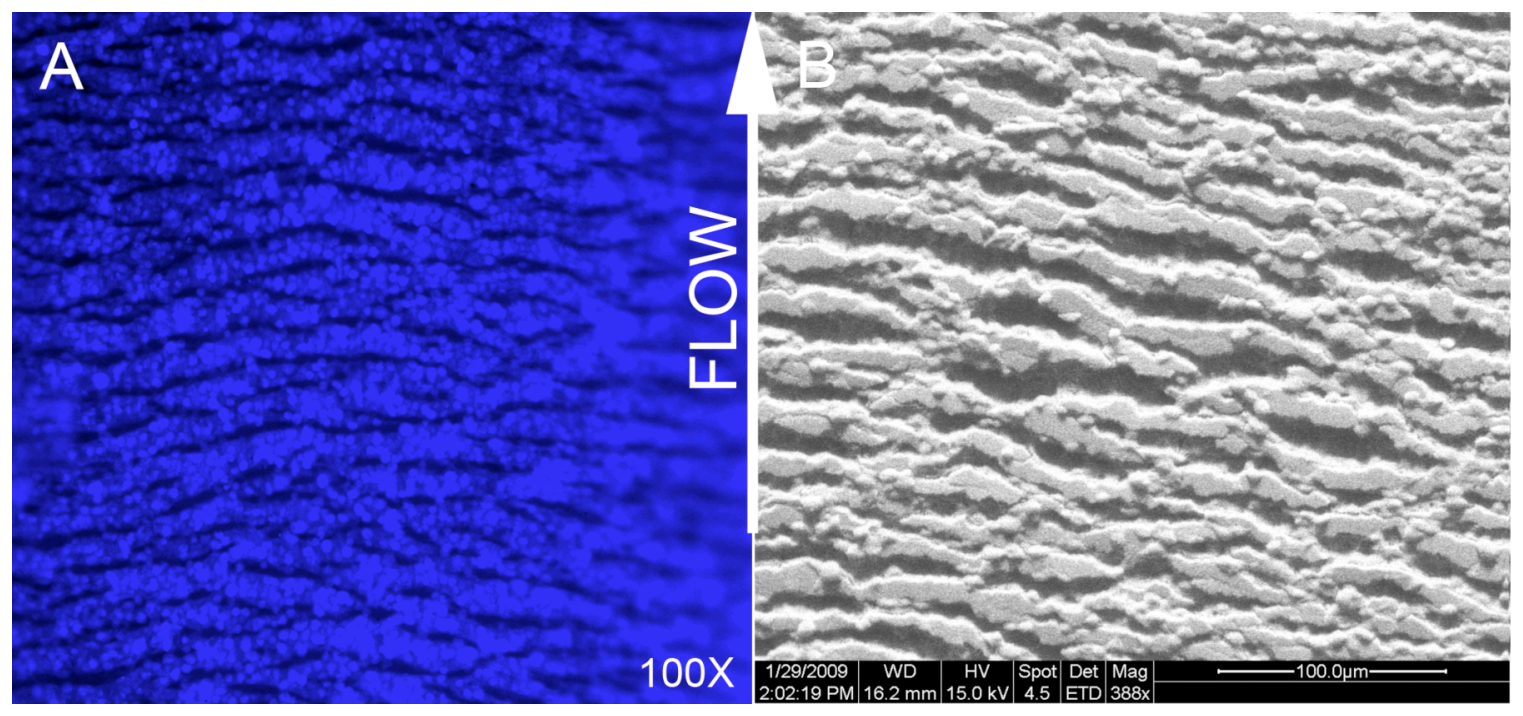

Figure 3.10 BBI(A) and SEM(B) imaging of construct SB2 (14\% Dextran, 28ml/min $6.3982 \mathrm{dyn} / \mathrm{cm}^{2}$ for 8 hours). 
Construct SB3 was exposed to the most rigorous conditions as it experienced $\sim 6.4$ $\mathrm{dyn} / \mathrm{cm}^{2}$ for 24 hours. Analysis revealed that the cells were retained on the scaffold and although the BBI image indicates a similar number of nuclei as the SB1 construct, the surface density and morphology were somewhat different. The cells on this scaffold appeared slightly more spread out and there was an apparent increase in the degree of cell extension and/or matrix deposition as indicated by the fibers along and across the scaffold node (Figure 3.11). For more cell surface images See Appendix A.

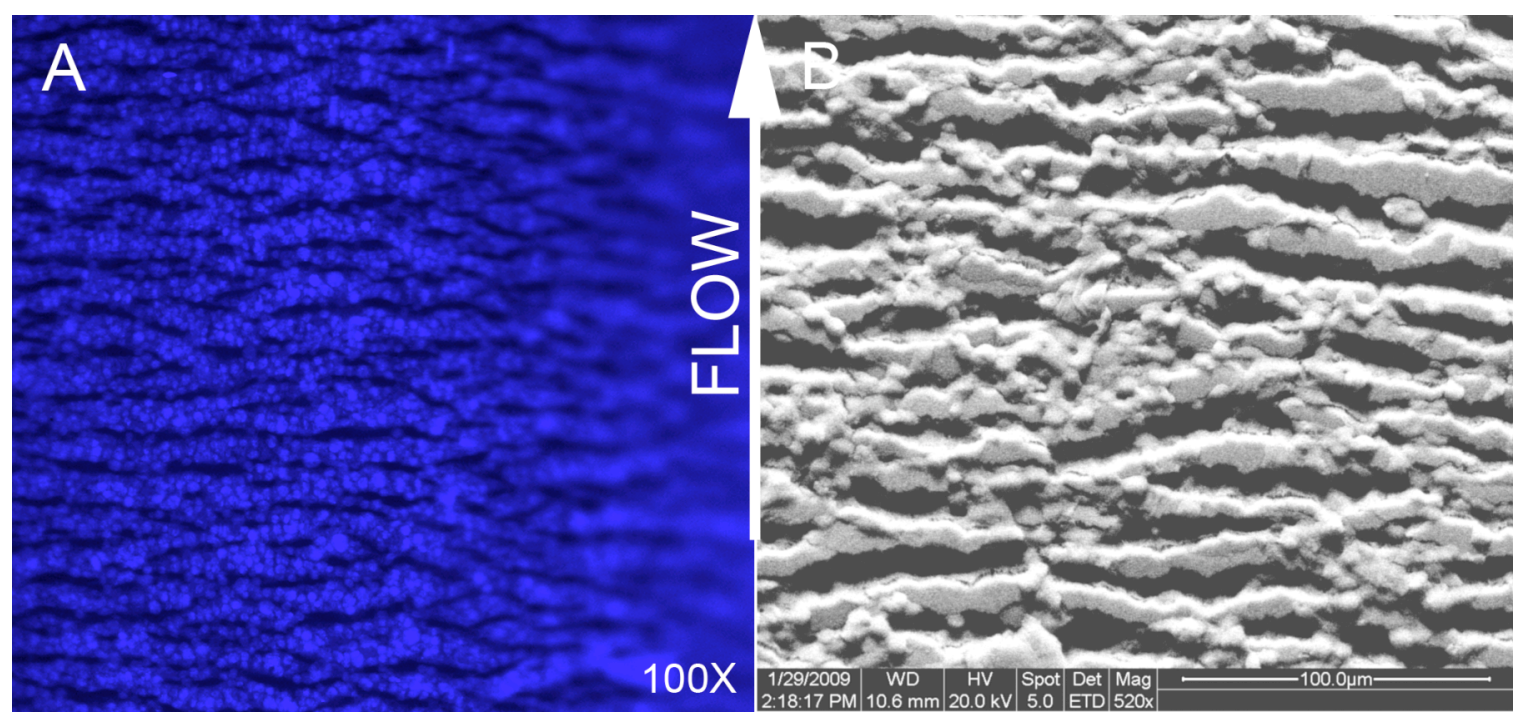

Figure 3.11 BBI(A) and SEM(B) imaging of construct SB3 (14\% Dextran, 28ml/min, $6.3982 \mathrm{dyn} / \mathrm{cm}^{2}$ for 24 hours).

\section{$\underline{\text { DISCUSSION }}$}

As with the implementation of pulsatile flow in the BVM bioreactor system, the main goal of the work presented in this chapter was to create wall shear stress at the inner surface of the graft with the intention of creating an environment in which the phenotype and orientation of the sodded cells would be improved over the previously configured system. Consistent with the overall intent of this system as a high throughput, in vitro coronary stent testing device, the creation of wall shear stress was approached with ease 
of use and scalability in mind. As shear stress had not yet been implemented in this system, the primary goals of this part of this thesis were to devise a simple method for doing so and to subsequently determine whether or not the applied conditions were conducive to cultivation of a cell-lined BVM.

The original configuration of the BVM system was not developed with appropriate wall shear stress in mind and after testing the viscosity of the media, it was found that the shear stress experienced by cells grown in the original system was $\sim 0.35$ $\mathrm{dyn} / \mathrm{cm}^{2}$, a figure well below the desired minimum of $6 \mathrm{dyn} / \mathrm{cm}^{2}$. The immediate thought when conceiving a method for increasing this figure was to simply increase the viscosity of the media to a level that would invoke desired shear stresses while keeping in mind the importance of not affecting cellular growth with said additive. It was also realized that the flow rate may need to be adjusted to achieve the desired shear stress, however this was to be used secondary to increasing the viscosity as it would further change the original configuration of the system.

\section{Development of Wall Shear Stress}

Contrary to the development process and preliminary testing involved in the pulsatile flow section of this thesis, the physical implementation of wall shear stress into the system was less demanding, as it required a simple change in the media used. The original concept was to adulterate the perfusion media to increase the viscosity and therefore the resulting shear stress placed on the inner walls of the BVM scaffold. Although there were many initial ideas for potential additives that could achieve this, background research prompted the decision to investigate methylcellulose and dextran for 
use in our system. However, due to the inconvenience of using methylcellulose in our media, it was abandoned as an option and testing was carried out using dextran only.

After determining the viscosities of various concentrations of dextran, the resulting shear stresses at different flow rates in the $4 \mathrm{~mm}$ diameter graft were calculated. As these measurements and calculations were rather straightforward, the difficulty posed by this portion of the thesis pertained primarily to deciding what combination of media viscosity and flow rate would provide the needed shear stress without bringing harm to the cells.

\section{Effect of Dextran on HUVEC Cultures}

Various concentrations of dextran in the bioreactor media were tested in static cultures of HUVECs to determine if and at what concentration the culture would be hindered simply by the presence of the dextran itself. Before carrying out this test, shear stresses were calculated at various dextran concentrations and flow rates. Using this data, the desired concentration of $14 \%$ dextran was selected as it was thought that it would not be too high for the wellbeing of the cells and it would provide the desired shear stress without requiring an excessively high flow rate. This was supported by the results of the static culture test as cultures grown in up to $14 \%$ dextran did not show significantly greater cell loss than those grown in lower concentrations. Although, the cultures grown in 20 and $30 \%$ dextran did show significant cell loss compared to those grown in $14 \%$, it is unknown how the cells would respond to media consisting of dextran concentrations in the $15-19 \%$ range. The effects of these additional formulations would require further 
investigation, which may have been warranted if lower flow rates and/or higher shear stress are desired.

\section{$\underline{\text { Scalability }}$}

As developed here, the method for implementing appropriate wall shear stress in the BVM system did not alter the overall physical configuration of the system to any degree. The bioreactor, tubing, scaffold, and peristaltic pumps were configured exactly as presented in the original design. The only changes were to the media formulation and the pump speed required during cultivation. Based on this statement, the physical scalability of this system with desired shear stress is exactly as it was with the original system. However, based on observations during the growth tests performed here, there may be a concern during long-term trials. Due to the increased pump speed required to achieve the necessary $28 \mathrm{ml} / \mathrm{min}$ there may be an issue with reliability of the pump tubing as cracks developed at the pump interface that resulted in minor leaks. This was not problematic in the relatively short trials presented here, however, a longer growth period may result in excessive media loss and/or culture contamination. This issue may be addressed with the implementation of more robust tubing.

\section{Comparison to Previous Work}

Due to its importance, the effects of shear stress on the growth and orientation of endothelial cells has been extensively investigated $[18,37,42,45,48,74,76,86]$. Several groups have succeeded in developing perfusion bioreactor systems used to cultivate TEVGs that apply appropriate shear levels using methods similar to those seen here 
$[25,28,37]$. Therefore, the achievement regarding the implementation of shear stress presented in this thesis is not unique when compared to the work of other groups in the field, but rather a means of confirming its validity in our system. The results presented here are valuable as the goal was not to develop novel methodology, but rather to implement shear stress conditions for the proper development of a native-like endothelial layer in the BVM. The novel aspect of this work is in the information provided regarding the variety of dextran concentrations and the respective viscosities and corresponding shear stresses at different flow rates, as this was not found to be available in previously published data. With this said, the work presented in this thesis should be evaluated as a novel condition specific to this BVM system and as groundwork for future development of an ideal BVM system.

\section{Cell Adhesion}

After determining the necessary parameters for achieving specific shear stress levels at the graft surface, the final phase of this portion of this thesis was to determine whether or not a HUVEC-sodded BVM could maintain cell adhesion with an increase in shear forces. Although the number of grafts cultivated in this investigation does not allow for claims that can be considered statistically significant regarding the best configuration, it has been demonstrated that shear stresses previously shown to improve EC growth can be implemented into this BVM system while maintaining cell adhesion. As mentioned above, although establishing shear stress in a bioreactor system such as this is not a novel achievement, some degree of accomplishment can be credited to the cells 
remaining on the scaffold under increased shear on an ePTFE scaffold, as this material is not highly conducive to cell adhesion.

\section{Construct Appearance and Cell Morphology}

As shown in Figure 3.7-Figure 3.11, the analysis of the constructs indicated that cells remained on the graft following exposure to all of the various shear stress conditions applied in this work. This was confirmed with the presence of nuclei as well as visualization under SEM. Although simply maintaining cell adhesion was the primary goal and measure of success in these trials, more critical examination showed that the cells exhibited an undesirable morphology and subsequently did not form a confluent monolayer on the graft surface. For an example of the desired surface appearance of a BVM cultivated in this system, see the micrograph of the HMVECs sodded construct cultivated in a previous study (Figure 3.12).

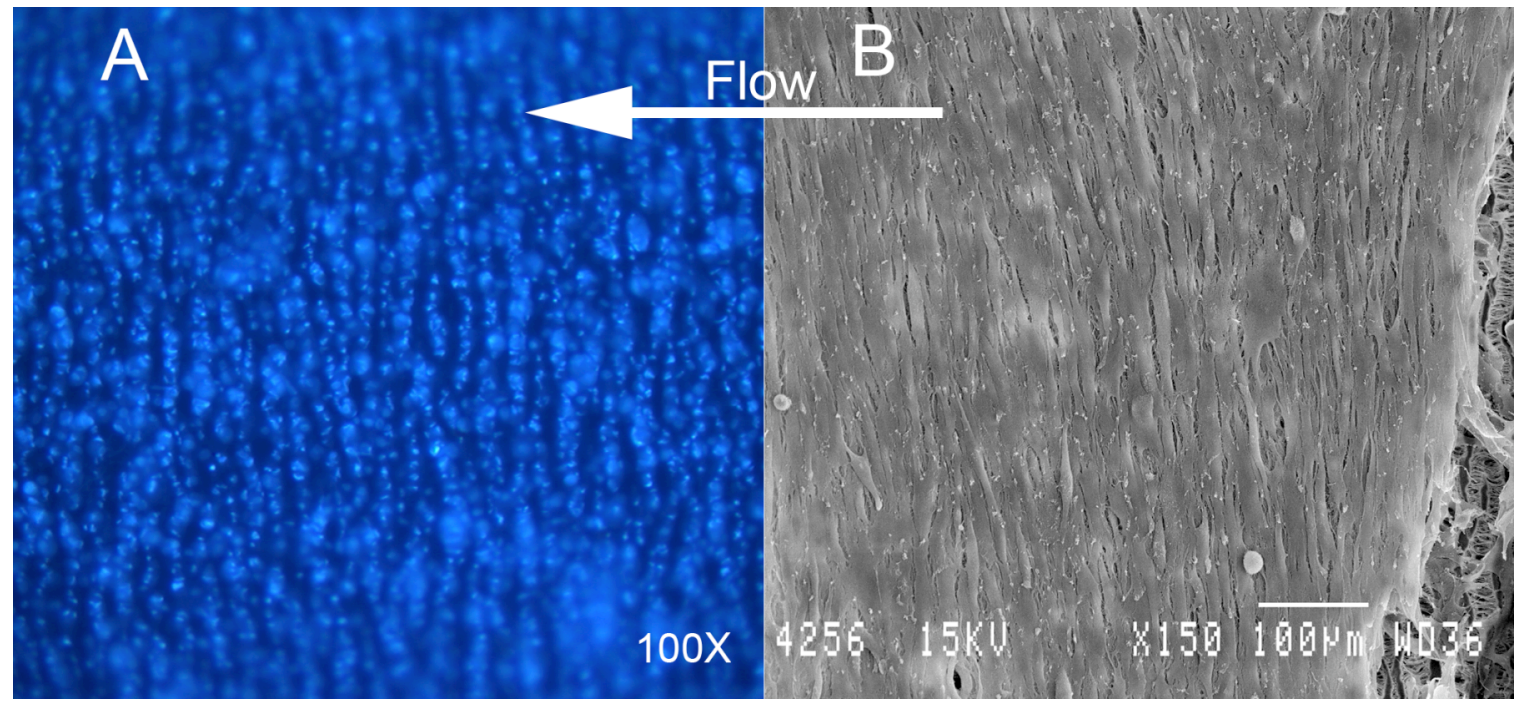

Figure 3.12 BBI(A) and SEM(B) images of ePTFE graft sodded with HMVECs $(0 \%$ Dextran, $15 \mathrm{ml} / \mathrm{min} 0.3592 \mathrm{dyn} / \mathrm{cm}^{2}$ ). K. Cardinal, unpublished data. 
As discussed in Chapter 2, the surface morphology seen in constructs cultivated for this thesis may have been due to the inherent nature of HUVECs and therefore not a direct result of the applied shear conditions. This possibility is backed by the fact that the cell morphology found on the control construct (SA1), which did not experience shear stress of any kind, was similar in appearance to those exposed to increased shear. When compared to the micrograph of HMVECs, which was exposed to identical conditions as SA1, the resulting appearance of all constructs cultivated in this trial can be considered dissimilar. Therefore, the fact that the control construct in this trial was similar in appearance to the variable groups, but dissimilar to the expected results seen in Figure 3.12, it cannot be concluded that the undesired morphology are a direct result of the changes made in this work.

Although the claim that the unfavorable morphology may be due to the HUVEC cell type cannot be backed by the results presented here, the results of several other groups' studies into the behavior of the HUVEC cell type may provide some explanation and/or ideas for potential improvements to the protocol used to cultivate HUVEC sodded BVM constructs with and without shear $[32,47,51]$. Further investigation is required to determine whether or not the method of implementing shear stress developed in this thesis can be used to cultivate a BVM with desirable morphology and surface confluence. 


\section{CHAPTER 4: DISCUSSION AND CONCLUSIONS}

\section{OVERVIEW}

The primary goal of this thesis was to develop, implement and test the feasibility of establishing physiologic flow conditions in a bioreactor system used to cultivate Blood Vessel Mimics (BVMs) in an effort to contribute to the overall goal of creating an ideal BVM construct. For purposes of this system, the development of an "ideal" setup would maintain its simple, high-throughput configuration, while making changes to the original design that will produce a construct with native-like qualities. The work presented here proposes methods for introducing both pulsatile flow and levels of shear stress that are intended to improve the phenotypic expression and therefore scientific value of the BVM system. Two key changes other than those presented in this thesis involve replacing the ePTFE scaffold with one that exhibits more native-like compliance and improved cell adhesion properties along with developing a method for sodding the graft with cell types that will create a construct with native-like layering. These two alterations are currently under development and although they are not discussed extensively in this thesis, they are necessary to fully realize the benefit of the work presented herein.

As discussed previously, the introduction of physiologic pulsatile flow and increased wall shear stress will improve the development of sodded cells in the BVM bioreactor system. The pressure fluctuations resulting from pulsatile flow create radial hoop stresses and an ensuing strain intended to improve the cell orientation and layer composition of the BVM construct. Although this has been shown to improve the growth of ECs, the benefit of this feature has been primarily noticed in the tunica media, or layer 
containing smooth muscles cells $[28,34,57]$. Appropriate levels of wall shear stress, on the other hand will primarily benefit the development of the EC layer as it has been shown to induce proper cell alignment as well as phenotypic and growth factor expression $[37,45,48]$. The work presented here aimed to show that these conditions could be introduced in a previously established system in a way that would allow for cell adhesion, while maintaining the system's original simplicity.

Although the results of the cell adhesion tests were suboptimal with regard to cell morphology in both the pulsatile flow and shear stress trials, the principle goal of maintaining some degree of cell adhesion was a success. Secondary to maintaining adhesion, cell morphology and graft surface structure showed a need for improvement. The studies in this thesis were intended to establish groundwork for future development and experimentation, and in this respect, are viewed as a success. Based on the experiences and results from this work, several aspects of the system were identified as potential targets for future studies that can optimize the results of BVM constructs cultivated in this system.

\section{FUTURE WORK}

\section{Pulsatile Flow}

The work performed in this thesis regarding the development of pulsatile flow was successful in that a simple, minimally-modified variation of the original system was used to cultivate an endothelial cell-sodded BVM construct while maintaining some degree of cell adhesion. However, the resulting system and the construct it was used to produce showed some need for improvements. 
As mentioned previously, the tunability of the pulsatile pressure conditions could be improved. This can be addressed with three objectives in mind: (1) providing a more precisely indexed and adjustable pressurization method that will allow for minute, gradual adjustments to the system pressure independent of flow rate, (2) establishing a consistent baseline pressure that simulates a native diastolic pressure level, and (3) optimization of the pressure ramp-up protocol.

To improve the tunability of the system pressure, the method for applying backpressure could be enhanced. Possible solutions could involve the incorporation of a flow controller design for this application or the placement of check valves inline with the system to reduce the tendency for back flow inherent to peristalsis.

The establishment of a simulated diastolic or baseline pressure has many potential solutions that may bring about many potential complications. The complexity seen in the bioreactor systems of other groups cultivating TEVGs is often due to the need to maintain this baseline pressure as the system must be very well sealed to prevent leaks and very well controlled. It is unknown how well the current system can maintain elevated pressures while still enduring the pressure spikes seen with pulsation. Because this change would add substantial complexity to a system meant to maintain simplicity, the need to do so should be investigated by determining whether or not adequate construct growth can be achieved by relying solely on the pulsed pressure without the application of the baseline pressure.

The protocol used to ramp up pressure was developed in an effort to gradually apply the desired pressure levels without disturbing the cells' growth or adhesion. This was established without any prior experience with methods that would provide the best 
possible results. Future studies could be developed to optimize the timing and rate of this increase.

Along with improving the qualities of pulsatile flow and the methods for creating it, there is potential for investigation into the use of pulsation in the cultivation of a SMC sodded construct. All cell adhesion tests performed in this thesis were carried out using endothelial cells and although pulsatile flow has been shown to benefit the phenotypic expression of ECs, it has also been shown to be a key aspect in the development of the SMC layer in particular $[10,41,58,62,68,69]$. For this to be fully explored, a different scaffold material would most likely be necessary. The current scaffold material, ePTFE, does not allow for much compliance and as a result, the degree of cyclic radial strain experienced by the cells is limited. As our laboratory aims to create an ideal BVM for intravascular device testing, the inclusion of SMCs and a more compliant scaffold are important endeavors that will allow for the full benefit of pulsatile flow to be realized. Currently, both of these needs are being addressed, as protocols for cultivating SMC sodded constructs and the creation of electrospun scaffolds are under development.

In addition to future work regarding the improvements upon the system as it is currently configured, the pulsatile flow of our system could also potentially be used to study the response of endothelial cells to varying degrees of pressure variation. This was briefly discussed in the Methods section of Chapter 2, as different settings were established that corresponded with different hypertensive conditions. These settings were not tested in a sodded BVM and the degree of cell adhesion under these conditions is unknown, however these settings can be achieved and are a potential target for future studies. 


\section{Wall Shear Stress}

In accordance with the initial goal of this work, a simple method for specifying and implementing appropriate levels of wall shear stress in an endothelial cell-sodded BVM was successfully developed, as discussed in Chapter 3 of this thesis. Furthermore, it was shown that these conditions could be used in the cultivation of a vascular construct while maintaining cell adhesion. Although this can be deemed a success for the purposes of this thesis, the work presented here reveals that further work is required to refine these conditions in the effort to produce an ideal BVM construct.

Unlike the apparent need to address the tunability of pulsatile flow, shear stress in the system can be easily and accurately adjusted to the desired level by varying the media viscosity and corresponding flow rate. These levels were characterized in this thesis, and further refinement of this characterization of flow conditions and resulting calculated shear levels is possible by employing more extensive principles of fluid dynamics. As discussed previously, several assumptions were made as to the flow conditions in the BVM scaffold that may have inadvertently disregarded certain characteristics that affect resulting shear levels. To address this, future investigations should determine whether the flow in the construct is entirely laminar or if there is some turbulence present as this has been shown to greatly affect the results of TEVG cultivation $[18,76]$.

In addition to addressing the possible presence and effect of turbulent flow on shear stress in the BVM system, there is a need to address the effect of pulsatile flow on the degree of shear stress experienced by the cells on the scaffold. For purposes of the shear stress studies performed in this thesis, the effect of pulsatility on wall shear stress was disregarded as the flow was considered to be steady and fully developed. However, 
due to the fact that the BVM system will eventually include pulsatile flow, its effects must be taken into account as pulsatility has been shown to enhance wall shear stress in a setup such as the BVM system [55]. To address this, others groups have included the Wormersley parameter in their shear stress calculations [25]. Once the effect of pulsatile flow on the calculated shear stress in the BVM system is fully understood, it can be easily tested by using media with the required viscosity in the 3-roller, pulsatile setup developed in chapter two of this thesis. Use of the 3-roller pump head will also help prevent the problems experienced in the shear stress cell adhesion trials, as the lower pump speed and wall thickness of the tubing reduce the chances for cracks to form.

Along with more accurately characterizing the flow in the BVM system, there is also a potential for improvement in the protocols used to implement shear stress. Items to address include: the concentration of dextran and corresponding flow rate used with the shear ramp-up protocol.

To achieve the desired minimum shear stress of $6 \mathrm{dyn} / \mathrm{cm}^{2}$, the perfusion media consisted of $14 \%$ dextran. Though this concentration was not found to hinder cellular growth to any significant degree, it is higher than concentrations used by other groups and the resulting construct did not exemplify the qualities and desired morphology that would be expected from exposure to these shear levels (e.g. cell alignment). This could be due to a number of different factors, however future investigations should focus on determining an optimal combination of dextran concentration and flow rate for use in the BVM bioreactor system.

In addition to optimizing the methods for adjusting shear stress levels, future work should determine the best possible way to implement shear in the BVM system. 
The protocols employed in the cell adhesion studies of Chapter 3 were intended to gradually increase shear stress in an effort to minimize cell loss that may occur with a sudden increase. However, this gradual increase may have actually increased cell loss and/or contributed to the observed undesired surface morphology as it has been shown that decreased shear levels can cause degradation and cell retraction in the endothelial layer of TEVGs and native blood vessels [46,48]. If this were the case, the resulting damage to the cell layer may not have been overcome by exposure to the desire minimum shear stress of $6 \mathrm{dyn} / \mathrm{cm}^{2}$ for 24 hours. Conversely, the ramp-up may need to be more gradual as the scaffold material, ePTFE, is inherently problematic in cell adhesion.

\section{Cell Culturing}

Other than improvements upon the system's flow characteristics and pressurization methods, the resulting cell morphology poses a need for possible change. As described in Chapters 2 and 3, the culturing characteristics of HUVECs may present a target for future investigations. There were three common procedures found that may be employed to improve the results of HUVEC cultivation. Based on studies by Inoguchi et al and Hsu et al on the morphology and surface retention of HUVECs, the first method that could be adopted involves an additional step following the sodding of cells onto the scaffold. Both the Inoguchi and Hsu found that incubating the sodded grafts in static conditions prior to introducing perfusive flow improved cell retention and spreading [31,32]. The second addition to the methods used when cultivating HUVECs in the BVM involves the growth media. The growth media used when culturing the cells statically in preparation for sodding contained a growth supplement specific to 
endothelial cells. This component was removed from the perfusion media used when cultivating the cells in the BVM. For improved morphology, it is suggested that HUVECs be cultured in the BVM with media containing ECGS. [20,47]. The final possible improvement to the cultivation procedure involves a change to the graft preparation. Inoguchi et al and Feugier et al found success in their respective studies by coating the interior surface of the graft with collagen prior to sodding them with cells $[20,32]$. This would provide a protein to which the cells may better adhere. Although these suggested changes to the procedures used in our lab might improve the qualities of the resulting constructs, they add an additional element of complexity to a system that is intended to be simple and high throughput and thus may be unnecessary.

An alternative to improving the results of cultivating HUVECs is to try using an endothelial cell from a different source such as HMVECs. Although there are many sources for ECs, our lab has extensive experience and success with HMVECs in particular and would thus eliminate the cells' growth characteristics as a variable during the development of pulsatile flow and shear stress in the BVM system. However, due to the fact that microvessels and not large arteries are the source for this cell type, our lab is trying to move away from using them in the BVM. HMVECs are more prone to differentiate than ECs from larger vessels and using a cell type such as HUVECs may produce a more native-like large artery in the BVM

In addition to optimizing the techniques for culturing human vascular cells used in the BVM system, there may be a need to explore the use of the same cell types from different species. This may present a valuable precursor to animal studies. Similarly, there may be a need to specify the genetic variability of the cell lines from which 
constructs are cultivated. Currently, BVM constructs are sodded with cells from a single cell line at a time. Although these cell lines are collected from a variety of donor sources, further specification and tracking of each cell line used could simulate the variability found in clinical human trials.

\section{Additional Future Work and Considerations}

Apart from designing future studies that aim to improve the methods developed in this thesis and other work intended to implement physiologic conditions in this BVM system to improve the outcome of the cultivated constructs, there are many potential subjects for future investigations using the BVM system as a tool. As discussed previously, the intended use for this model is as an in vitro means to investigate the cellular response to implanted coronary stents. However, because of its versatile design it can also be used to study other intravascular devices such as catheters used for coronary angioplasty as well as vascular response to blood vessel related conditions and drugs. As an example, our lab has used the BVM system to evaluate intravascular optical coherence tomography (OCT). These studies could be carried out with little or no modifications to the system as it exists now. A balloon catheter can be easily inserted and activated through the same port used to deploy a stent while a drug study might involve simply adding the subject pharmaceutical to the media at some point during cultivation.

Potential studies that could be facilitated by the work presented in this thesis include the effects and possible treatment options for vascular disease conditions. For example, the pulsatile flow setup could be used to investigate the effects of hypertension 
by increasing the system pressure to that which is seen in patients with this condition (as outlined in Table 2.2). Similarly, by altering wall shear stress using the methods developed here, the system can be used to study the potential for atherosclerotic plaque development with the correct cellular constructs in place. Along with simply studying cellular response to conditions such as these, they can be combined with drug or coronary stent studies to determine how the vessel will respond to a drug or the presence of a stent. Again, these studies cannot be used to replace in vivo studies, but they can be used as preliminary data that may enhance the drug or device development process.

Although some development is still required to produce an ideal BVM system, when that goal is achieved it may prove to be a valuable tool in the drug and device industry. Along with providing a device or drug company with valuable results that may improve their development process, a valid test is important as it may be presented to the Food and Drug Administration or other governing bodies as preclinical data in Investigational Device Exemption (IDE), PreMarket Approval (PMA) or 510K applications $[28,48]$. Data presented in these applications are used to illustrate that the drug or device in question is safe and effective, and if a vascular construct with native characteristics can be produced using the BVM bioreactor system, there is potential use for it in this manner.

\section{CONCLUSION}

The ultimate goal of creating pulsatile flow and increasing wall shear stress in the BVM bioreactor system was successfully achieved. In accordance with the initial intentions of this thesis, this was performed without any major changes to the overall 
configuration of the system and as mentioned before, the results of this thesis can be used as groundwork for many future studies into the creation of an ideal BVM. Although our system is similar to those of several other groups that have developed TEVG bioreactor systems capable of producing high quality, native-like constructs, our BVM bioreactor system is superior in its simplicity and use as a high-throughput device testing model. As a result, this system has limitless potential for use as an in vitro model and with some development it may eventually provide a means of testing that could significantly benefit the medical device industry. 


\section{CITED REFERENCES}

1. Fluid Mechanics: Glossary. [cited 2008 August 13]; Available from: http://www.efunda.com/formulae/fluids/glossary.cfm.

2. Dynamic Viscosity Converter. [cited 2008 June 29]; Available from: http://www.unitconversion.org/unit_converter/viscosity-dynamic.html.

3. Balloon angioplasty and stent insertion, in Encyclopedia Britannica. 2008, Retrieved on November 15, 2008 from Encyclopedia Britannica Online: http://p2.www.britannica.com/EBchecked/topic-art/720793/95218/Balloonangioplasty-and-stent-insertion-In-a-coronary-artery-where.

4. Atherosclerotic Plaque, in Encyclopedia Britannica. 2008, Retrieved on November 15, 2008 from Encyclopedia Britannica Online: http://www.britannica.com/EBchecked/topic/40935/atherosclerotic-plaque.

5. American Heart Association. Cardiovascular Disease Statistics. [cited 2008 November 17]; Available from: http://www.americanheart.org/presenter.jhtml?identifier $=4478$.

6. American Heart Association. Atherosclerosis. [cited 2008 November 17]; Available from: http://www.americanheart.org/presenter.jhtml?identifier=4440.

7. American Heart Association. Bypass Surgery, Coronary Artery. [cited 2008 November 17]; Available from: http://www.americanheart.org/presenter.jhtml?identifier $=4484$.

8. American Heart Association. Heart Disease and Stroke statistics: 2008 Update At-a-Glance. 2008 [cited 2008 December 19]; Available from: http://www.americanheart.org/.

9. Bancroft, G.N., et al., Fluid flow increases mineralized matrix deposition in 3D perfusion culture of marrow stromal osteoblasts in a dose-dependent manner. Proceedings of the National Academy of Sciences, 2002.99(20): p. 12600-12605.

10. Barron, V., et al., Bioreactors for cardiovascular cell and tissue growth: a review. Annals of biomedical engineering, 2003. 31(9): p. 1017-1030.

11. Butler, D.L., S.A. Goldstein, and F. Guilak, Functional tissue engineering: the role of biomechanics. Journal of biomechanical engineering, 2000. 122: p. 570.

12. Cardinal, K.O.H., et al., Tissue-engineered vascular grafts as in vitro blood vessel mimics for the evaluation of endothelialization of intravascular devices. Tissue engineering, 2006. 12(12): p. 3431-3438. 
13. Center for Disease Control and Prevention. Heart Disease. 02/12/2009 [cited 2009 February 24]; Heart Disease Statistics]. Available from: www.cdc.gov/heartdisease.

14. Chaplin, M. Water Structure and Science. 2008 12/18/2008 [cited 2008 November 22]; Available from: http://www.lsbu.ac.uk/water/hyrhe.html.

15. Charles River. Products and Services Guide. [cited 2008 October 17]; Available from: http://www.criver.com/.

16. Chollette, M., Director, Software Technology \& Innovation, Cardiac Rhythm Management Division, St. Jude Medical. Phone Conversation 12/23/2008.

17. Dartevelle, S., Numerical and granulometric approaches to geophysical granular flows, Ph.D. thesis, , in Department of Geological and Mining Engineering, Michigan Technological University: Houghton.

18. Davies, P.F., et al., Turbulent fluid shear stress induces vascular endothelial cell turnover in vitro. Proceedings of the National Academy of Sciences, 1986. 83(7): p. 2114-2117.

19. Engineering Toolbox. Dynamic, Absolute and Kinematic Viscosity. [cited 2008 July 27]; Available from: http://www.engineeringtoolbox.com/dynamic-absolutekinematic-viscosity-d_412.html.

20. Feugier, P., et al., Attachment, morphology and adherence of human endothelial cells to vascular prosthesis materials under the action of shear stress. Biomaterials, 2005. 26(13): p. 1457-1466.

21. Fisslthaler, B., et al., Cyclic stretch enhances the expression and activity of coronary endothelium-derived hyperpolarizing factor synthase. Hypertension, 2001. 38(6): p. 1427-1432.

22. Fox, Stuart I., Human Physiology 4th Edition. 1992: Brown Publishers.

23. Fry, D.L., Acute vascular endothelial changes associated with increased blood velocity gradients. Circulation research, 1968. 22(2): p. 165-197.

24. Gallik, S., A. Bradshaw, and M. van Wambeck, Effects of methylcellulose on epithelial cells. In Vitro Cellular \& Developmental Biology-Animal, 1993. 29(10): p. 755-757.

25. Giudiceandrea, A., et al., Development and evaluation of an ideal flow circuit: assessing the dynamic behavior of endothelial cell seeded grafts. Journal of Artificial Organs, 2000. 3(1): p. 16-24.

26. Greenwald, S.E. and C.L. Berry, Improving vascular grafts: the importance of 
mechanical and haemodynamic properties. The Journal of Pathology, 2000. 190(3): p. 292-299.

27. Grewe, P.H., et al., Acute and chronic tissue response to coronary stent implantation: pathologic findings in human specimen. Journal of the American College of Cardiology, 2000. 35(1): p. 157-163.

28. Hahn, M.S., et al., Physiologic pulsatile flow bioreactor conditioning of poly (ethylene glycol)-based tissue engineered vascular grafts. Annals of biomedical engineering, 2007. 35(2): p. 190-200.

29. Hoerstrup, S.P., et al., Tissue engineering of small caliber vascular grafts. European Journal of Cardio-thoracic Surgery, 2001. 20(1): p. 164-169.

30. Holmes Jr, D.R., et al., Restenosis after percutaneous transluminal coronary angioplasty (PTCA): a report from the PTCA Registry of the National Heart, Lung, and Blood Institute. The American journal of cardiology, 1984. 53(12): p. 77C.

31. Hsu, S., et al., The effect of dynamic culture conditions on endothelial cell seeding and retention on small diameter polyurethane vascular grafts. Medical Engineering and Physics, 2005. 27(3): p. 267-272.

32. Inoguchi, H., et al., The effect of gradually graded shear stress on the morphological integrity of a huvec-seeded compliant small-diameter vascular graft. Biomaterials, 2007. 28(3): p. 486-495.

33. Isenberg, B.C., C. Williams, and R.T. Tranquillo, Small-diameter artificial arteries engineered in vitro. Circulation research, 2006. 98(1): p. 25-35.

34. Jeong, S.I., et al., Mechano-active tissue engineering of vascular smooth muscle using pulsatile perfusion bioreactors and elastic PLCL scaffolds. Biomaterials, 2005. 26(12): p. 1405-1411.

35. Kaplan, A.V., et al., Medical Device Development From Prototype to Regulatory Approval. Circulation, 2004. 109(25): p. 3068-3072.

36. Karamanoglu, M., A system for analysis of arterial blood pressure waveforms in humans. Computers and Biomedical Research, 1997. 30(3): p. 244-255.

37. Koller, A., D. Sun, and G. Kaley, Role of shear stress and endothelial prostaglandins in flow-and viscosity-induced dilation of arterioles in vitro. Circulation research, 1993. 72(6): p. 1276-1284. 
38. Kornowski, R., et al., In-stent restenosis: contributions of inflammatory responses and arterial injury to neointimal hyperplasia. Journal of the American College of Cardiology, 1998. 31(1): p. 224-230.

39. Ku, D.N., Blood flow in arteries. Annual Review of Fluid Mechanics, 1997. 29(1): p. 399-434.

40. Lakes, R. Meaning of Poisson's Ratio. [cited 2008 June 28]; Available from: http://silver.neep.wisc.edu/ lakes/PoissonIntro.html.

41. Laurent, S., et al., Local pulse pressure is a major determinant of large artery remodeling. Clinical and Experimental Pharmacology and Physiology, 2001. 28(12): p. 1011-1014.

42. Lee, A.A., et al., Fluid shear stress-induced alignment of cultured vascular smooth muscle cells. Journal of biomechanical engineering, 2002. 124: p. 37.

43. Liu, M.W., G.S. Roubin, and S.B. King, Restenosis after coronary angioplasty. Potential biologic determinants and role of intimal hyperplasia. Circulation, 1989. 79(6): p. 1374-1387.

44. Maintz, D., et al., 64-slice multidetector coronary CT angiography: in vitro evaluation of 68 different stents. European radiology, 2006. 16(4): p. 818-826.

45. Malek, A.M., Mechanism of endothelial cell shape change and cytoskeletal remodeling in response to fluid shear stress. Journal of Cell Science, 1996. 109(4): p. 713-726.

46. Malek, A.M., S.L. Alper, and S. Izumo, Hemodynamic shear stress and its role in atherosclerosis. JAMA, 1999. 282(21): p. 2035-2042.

47. Marin, V., et al., Endothelial cell culture: protocol to obtain and cultivate human umbilical endothelial cells. Journal of immunological methods, 2001. 254(1-2): p. 183-190.

48. Masuda, M. and K. Fujiwara, Morphological responses of single endothelial cells exposed to physiological levels of fluid shear stress. Front Med Biol Eng, 1993. 5(2): p. 79-87.

49. Mayo Clinic Staff. Cardiovascular disease 101: Understanding heart and blood vessel conditions. 2007 8/9/2007 [cited 2008 November 23]; Available from: http://www.mayoclinic.com/health/cardiovascular-disease/HB00032.

50. Mitchell, S.L. and L.E. Niklason, Requirements for growing tissue-engineered vascular grafts. Cardiovascular Pathology, 2003. 12(2): p. 59-64.

51. Morigi, M., et al., Fluid shear stress modulates surface expression of adhesion 
molecules by endothelial cells. Blood, 1995. 85(7): p. 1696.

52. Morton, A., R. Walker, and J. Gunn, Current challenges in coronary stenting: from bench to bedside. Biochem. Soc. Trans, 2007. 35: p. 900-904.

53. Mosenkis, R., Strategies for Bench Testing Medical Devices Careful planning is crucial when embarking on a medical device testing project. Choosing the appropriate tests, times, and facilities-while sticking to your testing goal-is key. MEDICAL DEVICE AND DIAGNOSTIC INDUSTRY, 2003. 25(4): p. 58-62.

54. Muschler, G.F., C. Nakamoto, and L.G. Griffith, Engineering principles of clinical cell-based tissue engineering. The Journal of Bone and Joint Surgery, 2004. 86(7): p. 1541-1558.

55. Nakata, M., et al., Augmentative effect of pulsatility on the wall shear stress in tube flow. Artificial Organs, 1999. 23(8): p. 727-731.

56. National Heart Lung and Blood Institute. Disease and Conditions Index. [cited; Available from: http://www.nhlbi.nih.gov/health/dci/Browse/Heart.html.

57. Niklason, L.E., et al., Morphologic and mechanical characteristics of engineered bovine arteries. Journal of vascular surgery: official publication, the Society for Vascular Surgery [and] International Society for Cardiovascular Surgery, North American Chapter, 2001. 33(3): p. 628.

58. Niklason, L.E., et al., Functional arteries grown in vitro. Science, 1999. 284(5413): p. 489.

59. Nobuyoshi, M., et al., Restenosis after percutaneous transluminal coronary angioplasty: pathologic observations in 20 patients. Journal of the American College of Cardiology, 1991. 17(2): p. 433-439.

60. O'Rourke, M.F., A. Pauca, and X.J. Jiang, Pulse wave analysis. British journal of clinical pharmacology, 2001. 51(6): p. 507.

61. Ofili, E.O., A.J. Labovitz, and M.J. Kern, Coronary flow velocity dynamics in normal and diseased arteries. The American journal of cardiology, 1993. 71(14): p. 3-9.

62. Popp, R., I. Fleming, and R. Busse, Pulsatile Stretch in Coronary Arteries Elicits Release of Endothelium-Derived Hyperpolarizing Factor A Modulator of Arterial Compliance. Circulation Research, 1998. 82(6): p. 696-703.

63. Pross, C., et al., Dexamethasone inhibits vascular smooth muscle cell migration via modulation of matrix metalloproteinase activity. Journal of Surgical Research, 2002. 102(2): p. 57-62. 
64. Ratcliffe, A., Tissue engineering of vascular grafts. Matrix Biology, 2000. 19(4): p. 353-357.

65. Ritchie, J.L., et al., Association between percutaneous transluminal coronary angioplasty volumes and outcomes in the Healthcare Cost and Utilization Project 1993-1994. The American journal of cardiology, 1999. 83(4): p. 493-497.

66. Roberts, H.W. and S.W. Redding, Coronary artery stents: Review and patientmanagement recommendations. The Journal of the American Dental Association, 2000. 131(6): p. 797-801.

67. Schmedlen, R.H., et al., Tissue engineered small-diameter vascular grafts. Clinics in plastic surgery, 2003. 30(4): p. 507-517.

68. Seliktar, D., et al., Dynamic mechanical conditioning of collagen-gel blood vessel constructs induces remodeling in vitro. Annals of biomedical engineering, 2000. 28(4): p. 351-362.

69. Seliktar, D., R.M. Nerem, and Z.S. Galis, Mechanical strain-stimulated remodeling of tissue-engineered blood vessel constructs. Tissue engineering, 2003. 9(4): p. 657-666.

70. Sodian, R., et al., Tissue-engineering bioreactors: a new combined cell-seeding and perfusion system for vascular tissue engineering. Tissue engineering, 2002. 8(5): p. 863-870.

71. Sousa, J.E., P.W. Serruys, and M.A. Costa, New frontiers in cardiology drugeluting stents: part I. Circulation, 2003. 107(17): p. 2274-2279.

72. Sousa, J.E., P.W. Serruys, and M.A. Costa, Clinical Cardiology: New FrontiersNew Frontiers in Cardiology: Drug-Eluting Stents: Part II. CirculationHagertown, 2003. 107(18): p. 2383-2389.

73. Stilson, T. Blood Vessels. [cited 2008 July 27]; Available from: http://facweb.northseattle.edu/tstilson/homepage/.

74. Stone, P.H., et al., Effect of endothelial shear stress on the progression of coronary artery disease, vascular remodeling, and in-stent restenosis in humans in vivo 6-month follow-up study. Circulation, 2003. 108(4): p. 438-444.

75. Thompson, C.A., et al., A novel pulsatile, laminar flow bioreactor for the development of tissue-engineered vascular structures. Tissue engineering, 2002. 8(6): p. 1083-1088.

76. Traub, O. and B.C. Berk, Laminar shear stress mechanisms by which endothelial 
cells transduce an atheroprotective force. Arteriosclerosis, thrombosis, and vascular biology, 1998. 18(5): p. 677-685.

77. U.S._Department_of_Health_and_Human_Services. Disease and Conditions Index. 2007 [cited 2008 November 11]; Available from:

http://www.nhlbi.nih.gov/health/dci/Browse/Heart.html.

78. University of Chicago Medical Center. Coronary Artery Bypass Surgery. [cited 2008 October 24]; Available from:

http://www.uchospitals.edu/specialties/heart/services/coronary-arterydisease/bypass/index.html.

79. Urakami-Harasawa, L., et al., Importance of endothelium-derived hyperpolarizing factor in human arteries. Journal of Clinical Investigation, 1997. 100(11): p. 2793-2799.

80. Vaughn Blood Pressure Chart. [cited 2008 June 17]; Available from: http://www.vaughns-1-pagers.com/medicine/blood-pressure.htm

81. Virmani, R., et al., Drug eluting stents: are human and animal studies comparable? British Medical Journal, 2003. 89(2): p. 133-138.

82. Weisstein, E.W. Shear Stress. 2007 [cited 2008 October 23]; Available from: http://scienceworld.wolfram.com/physics/ShearStress.html.

83. Wentzel, J.J., et al., Relationship between neointimal thickness and shear stress after Wallstent implantation in human coronary arteries. Circulation, 2001. 103(13): p. 1740-1745.

84. Williams, B., Mechanical influences on vascular smooth muscle cell function. Journal of hypertension, 1998. 16(12): p. 1921.

85. Williams, C. and T.M. Wick, Perfusion bioreactor for small diameter tissueengineered arteries. Tissue engineering, 2004. 10(5-6): p. 930-941.

86. Yamamoto, K., et al., Proliferation, differentiation, and tube formation by endothelial progenitor cells in response to shear stress. Journal of Applied Physiology, 2003. 95(5): p. 2081-2088.

87. Yock, C.A., et al., Cost-effectiveness of bypass surgery versus stenting in patients with multivessel coronary artery disease. The American Journal of Medicine, 2003. 115(5): p. 382-389.

88. Zhang, Q., et al., Neointimal hyperplasia persists at six months after sirolimuseluting stent implantation in diabetic porcine. Cardiovasc Diabetol, 2007. 6: p. 16. 
89. Ziegler, T. and R.M. Nerem, Tissue engineering a blood vessel: Regulation of vascular biology by mechanical stresses. Journal of cellular biochemistry, 1994. 56(2). 


\section{APPENDIX A: Additional Figures}

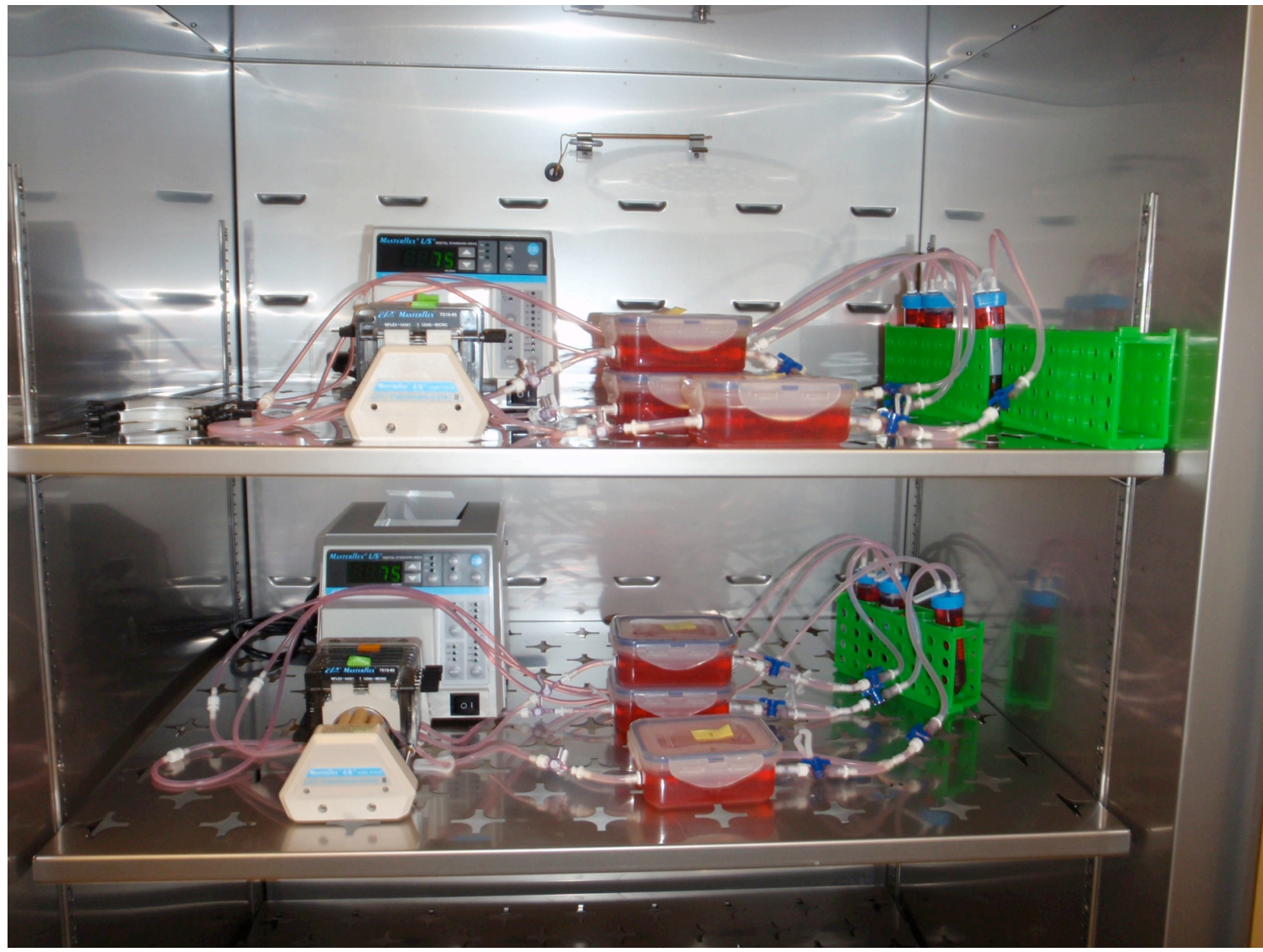

Figure A.1 BVM bioreactors in incubator setup for wall shear stress cell adhesion trial. 


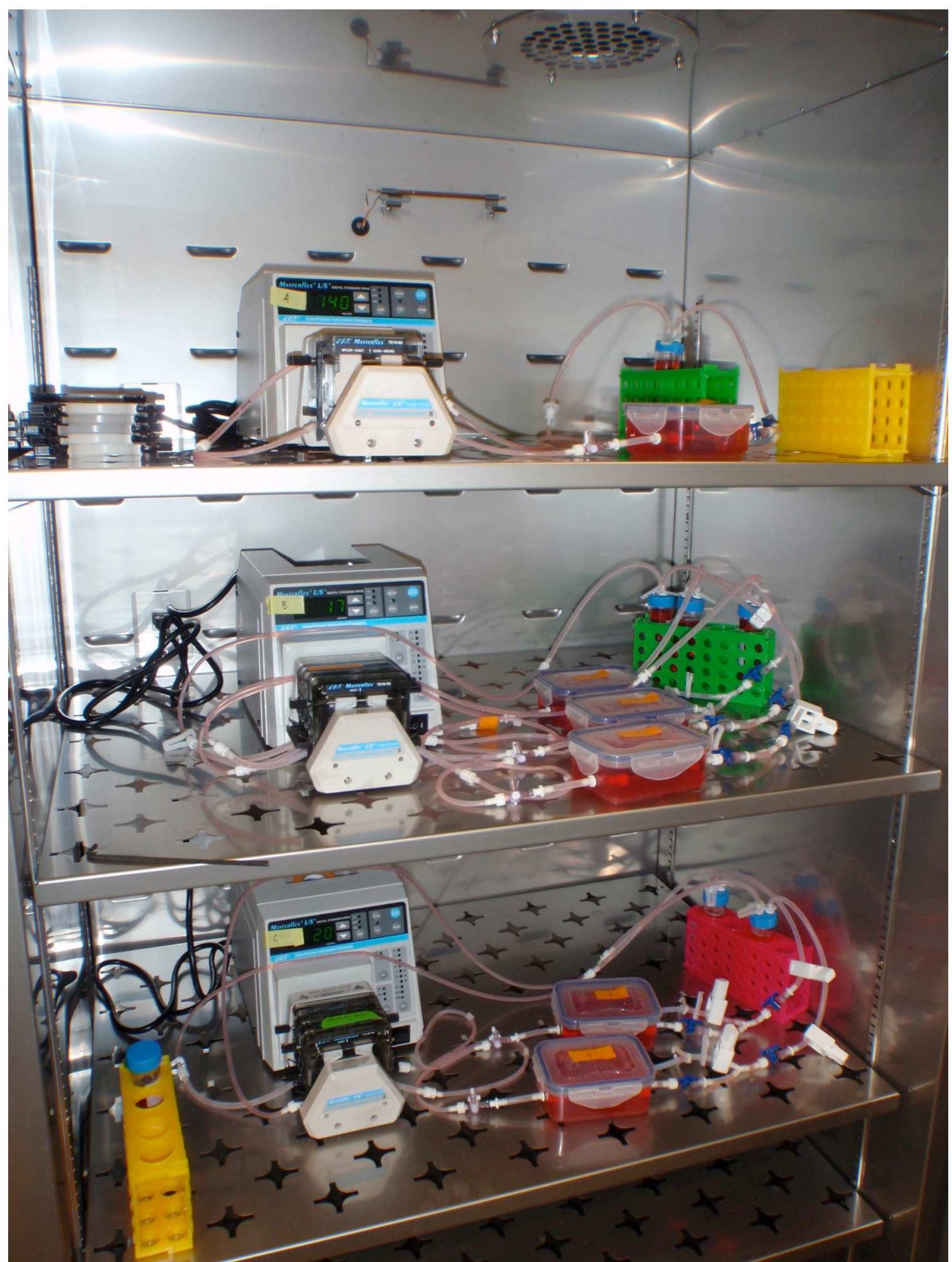

Figure A.2 BVM bioreactors in incubator setup for pulsatile flow cell adhesion trial. 


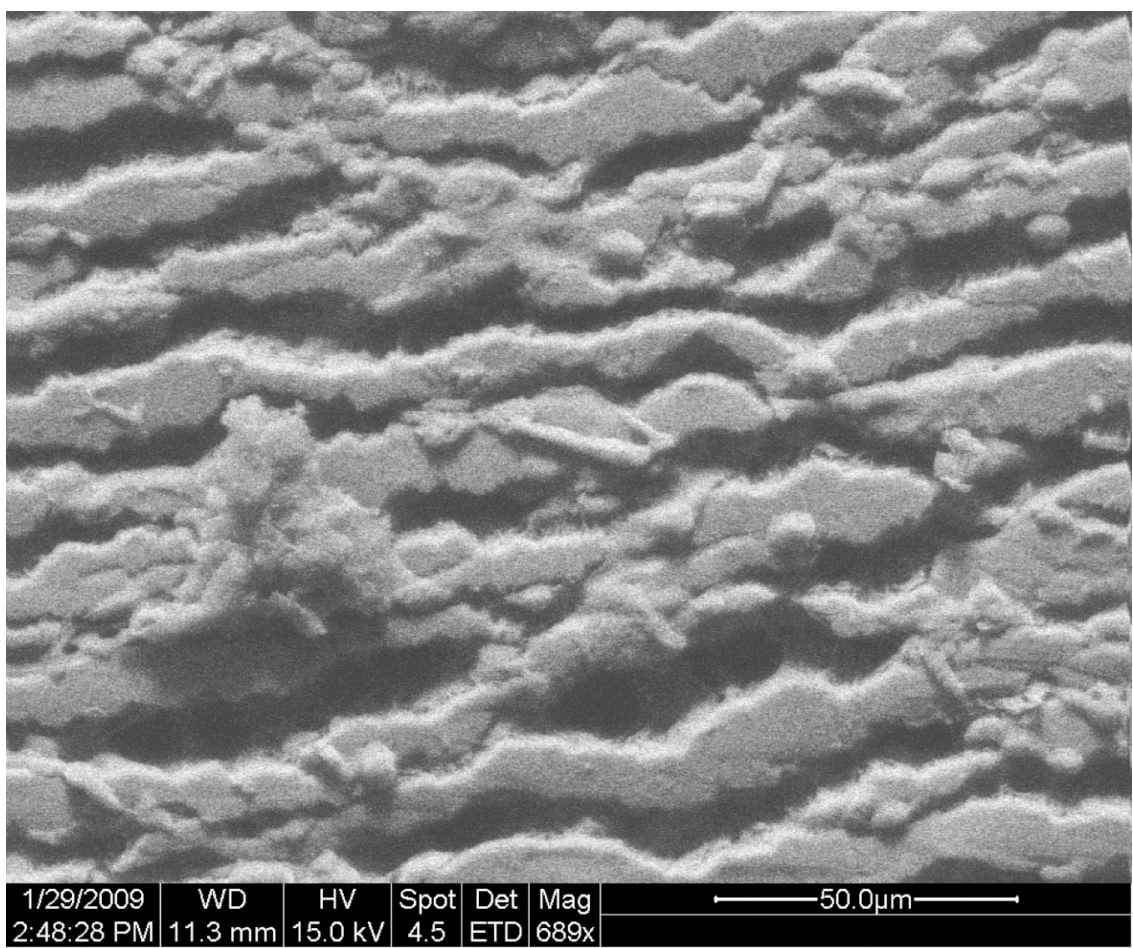

Figure A.3 SEM image of construct PB1 (72 hours of steady flow on 8-roller pump (a) $15 \mathrm{ml} / \mathrm{min}$ and 24 hours of steady flow on 3-roller pump).

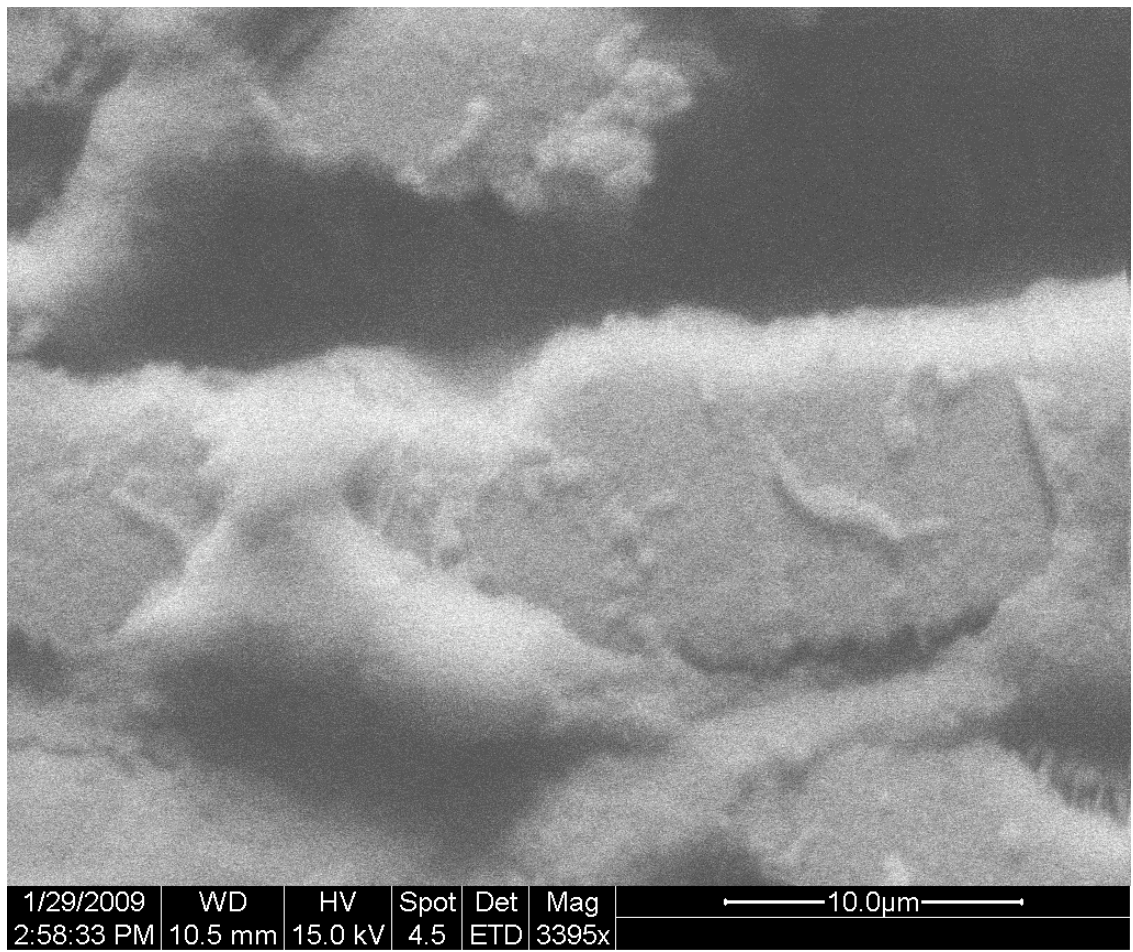

Figure A.4 High magnification SEM image of HUVEC on construct PB2 (72 hours of steady flow on 8-roller pump@ 15ml/min and 24 hours of pulsatile flow on 3roller pump at $18.5 \mathrm{ml} / \mathrm{min}$ and $\sim 40 \mathrm{mmHg}$ ). 


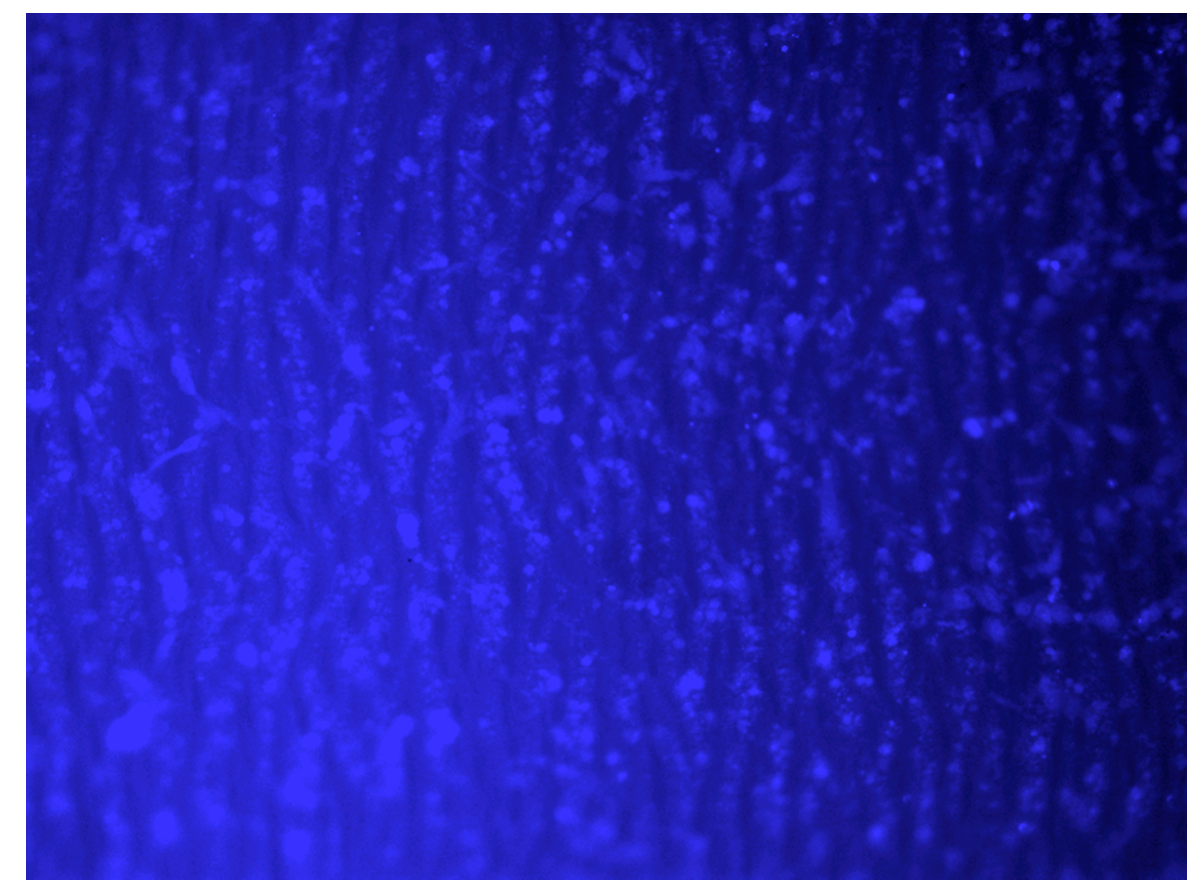

Figure A.5 BBI image of construct PB3 (72 hours of steady flow on 8-roller pump @ $15 \mathrm{ml} / \mathrm{min}$ and 24 hours of pulsatile flow on 3-roller pump at $18.5 \mathrm{ml} / \mathrm{min}$ and $\sim 40 \mathrm{mmHg}$ ).

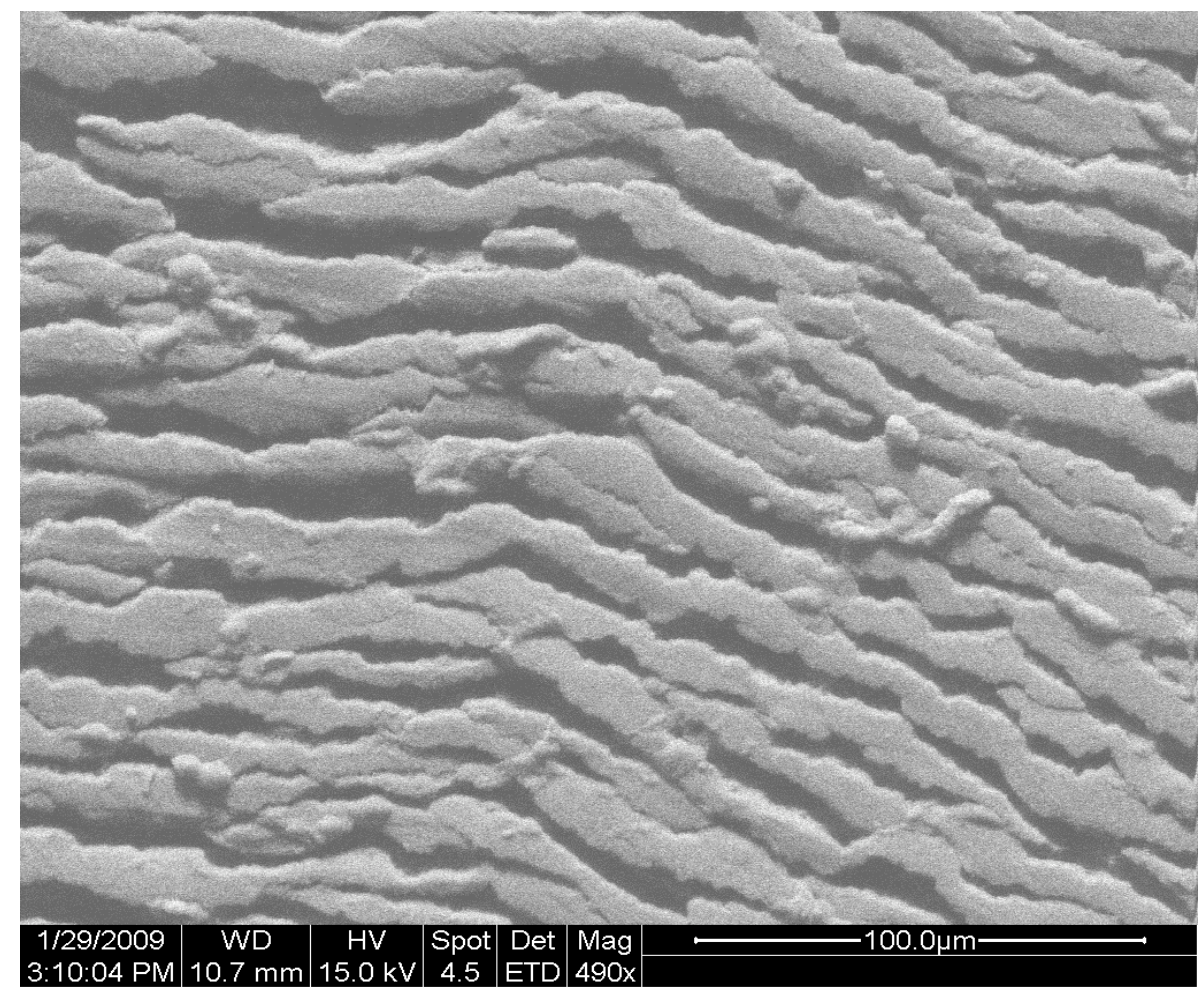

Figure A.6 SEM image of construct PB3 (72 hours of steady flow on 8-roller pump @ $15 \mathrm{ml} / \mathrm{min}$ and 24 hours of pulsatile flow on 3 -roller pump at $18.5 \mathrm{ml} / \mathrm{min}$ and $\sim 40 \mathrm{mmHg}$ ). 


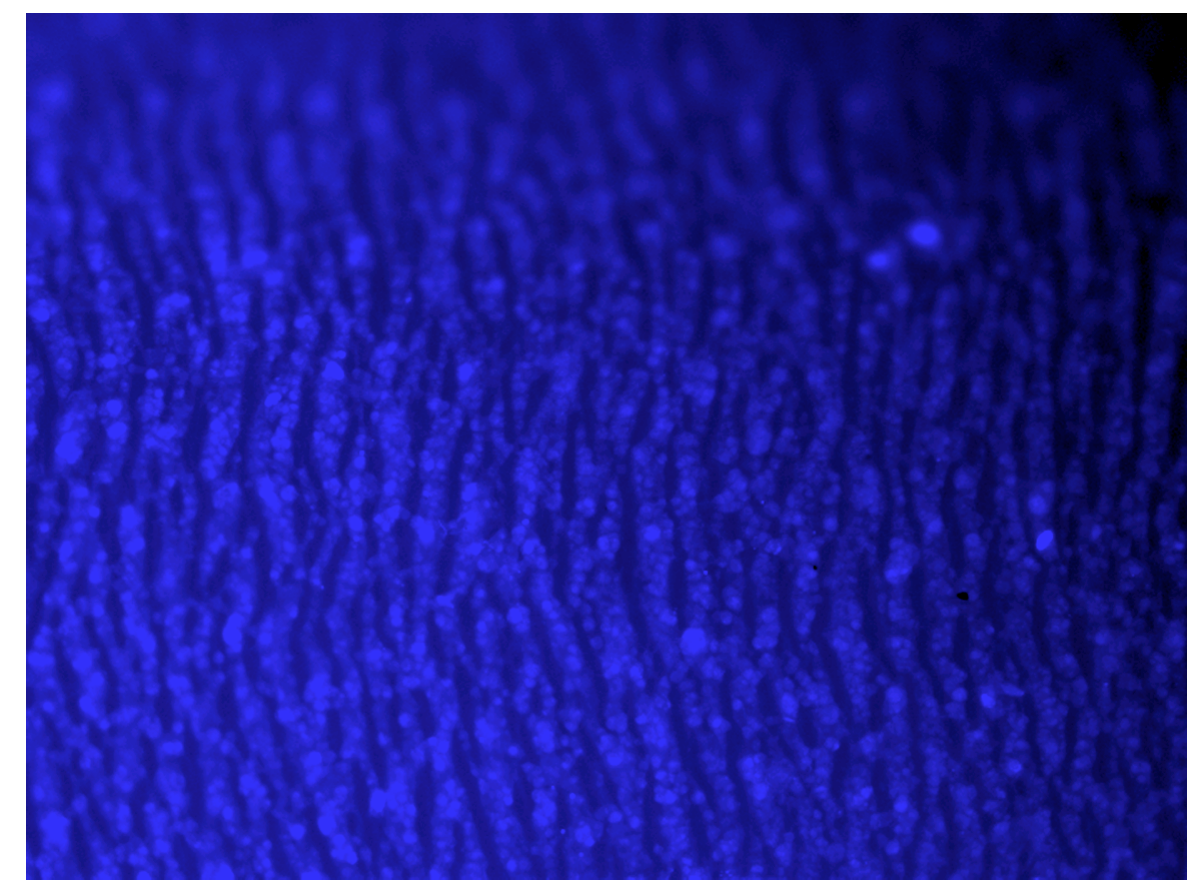

Figure A.7 BBI image of construct PB4 (72 hours of steady flow on 8-roller pump @ $15 \mathrm{ml} / \mathrm{min}$ and 24 hours of pulsatile flow on 3-roller pump at $18.5 \mathrm{ml} / \mathrm{min}$ and $\sim 80 \mathrm{mmHg}$ ).

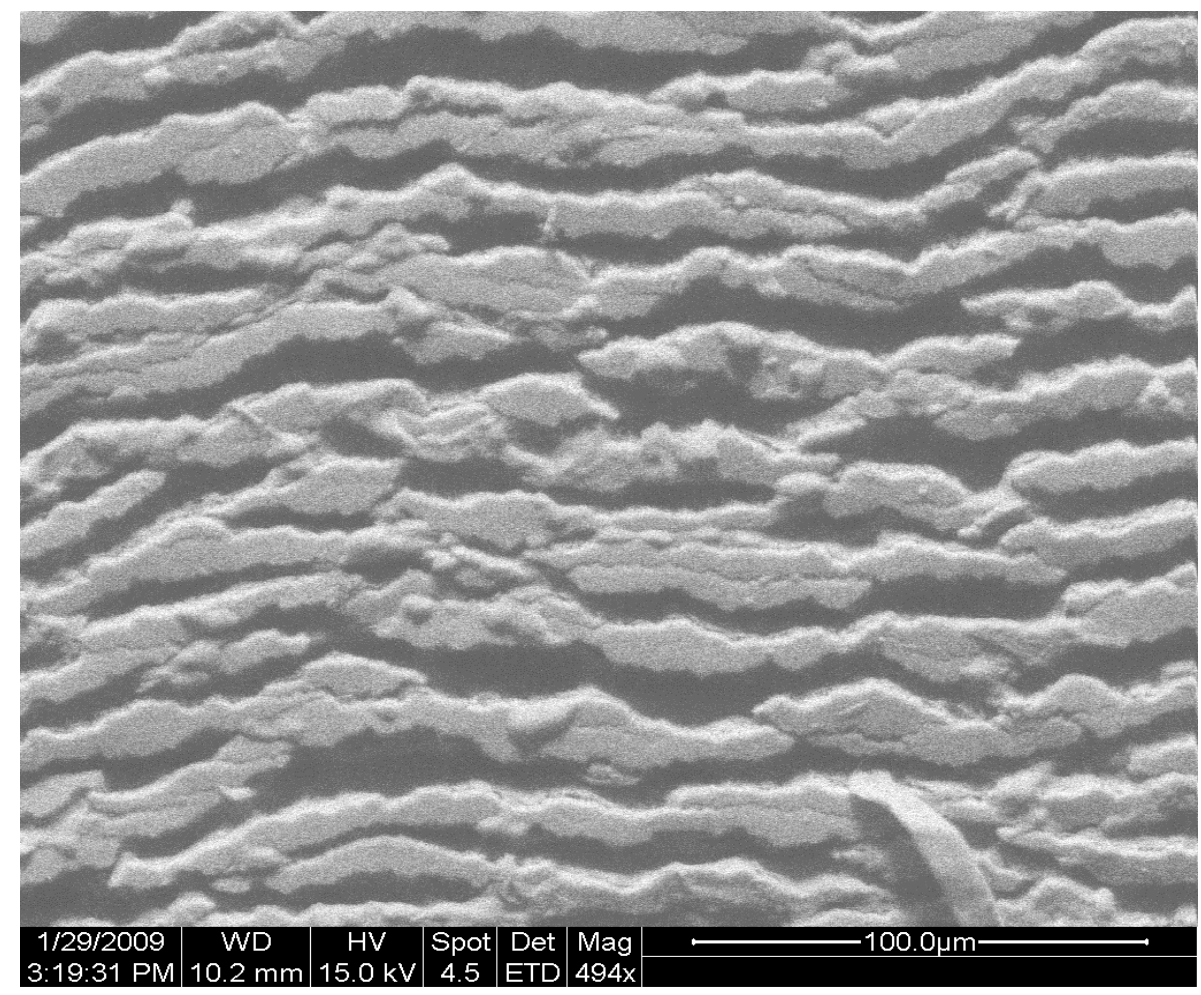

Figure A.8 SEM image of construct PB4 (72 hours of steady flow on 8-roller pump @ $15 \mathrm{ml} / \mathrm{min}$ and 24 hours of pulsatile flow on 3 -roller pump at $18.5 \mathrm{ml} / \mathrm{min}$ and $\sim 80 \mathrm{mmHg}$ ). 


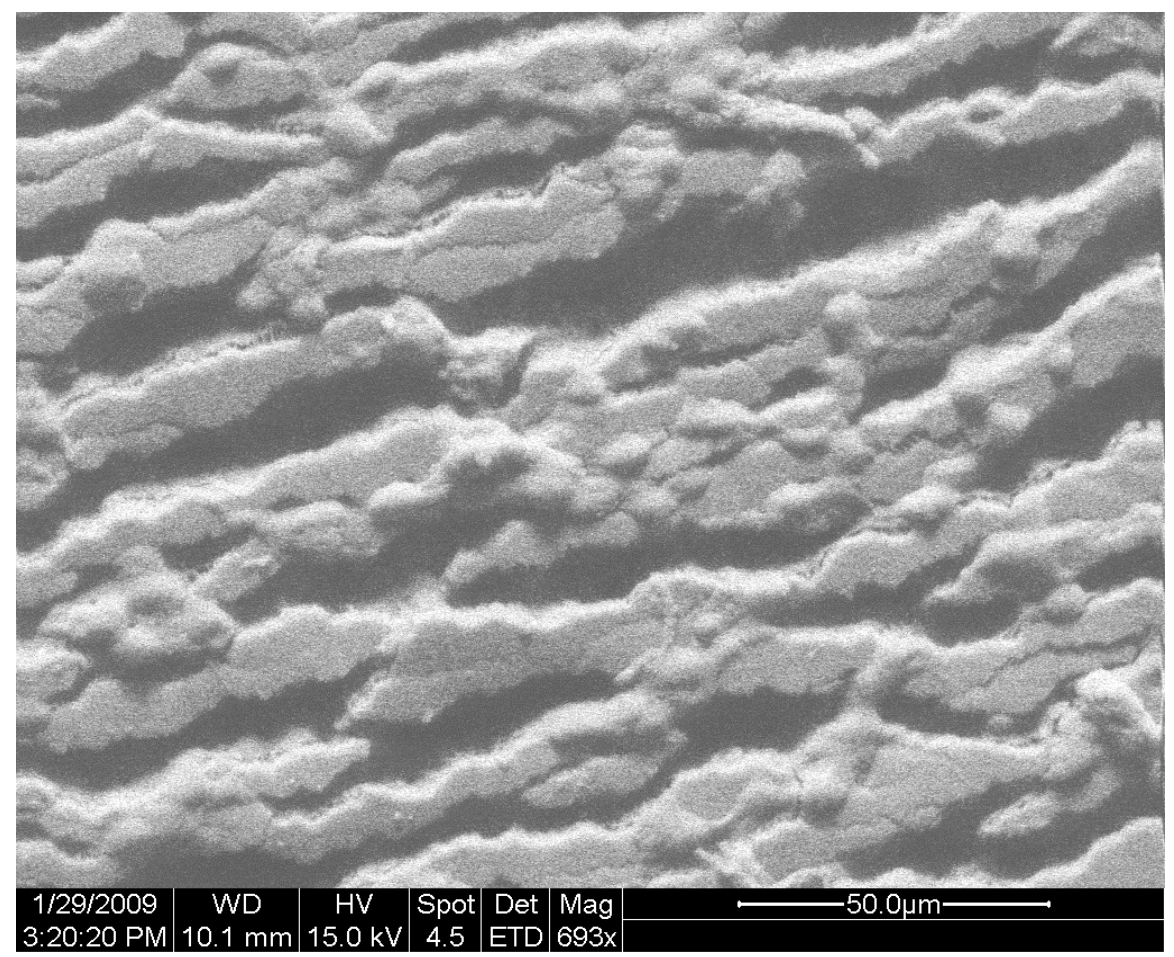

Figure A.9 Higher magnification SEM image of construct PB4 (72 hours of steady flow on 8-roller pump @ 15ml/min and 24 hours of pulsatile flow on 3-roller pump at $18.5 \mathrm{ml} / \mathrm{min}$ and $\sim 80 \mathrm{mmHg}$ ).

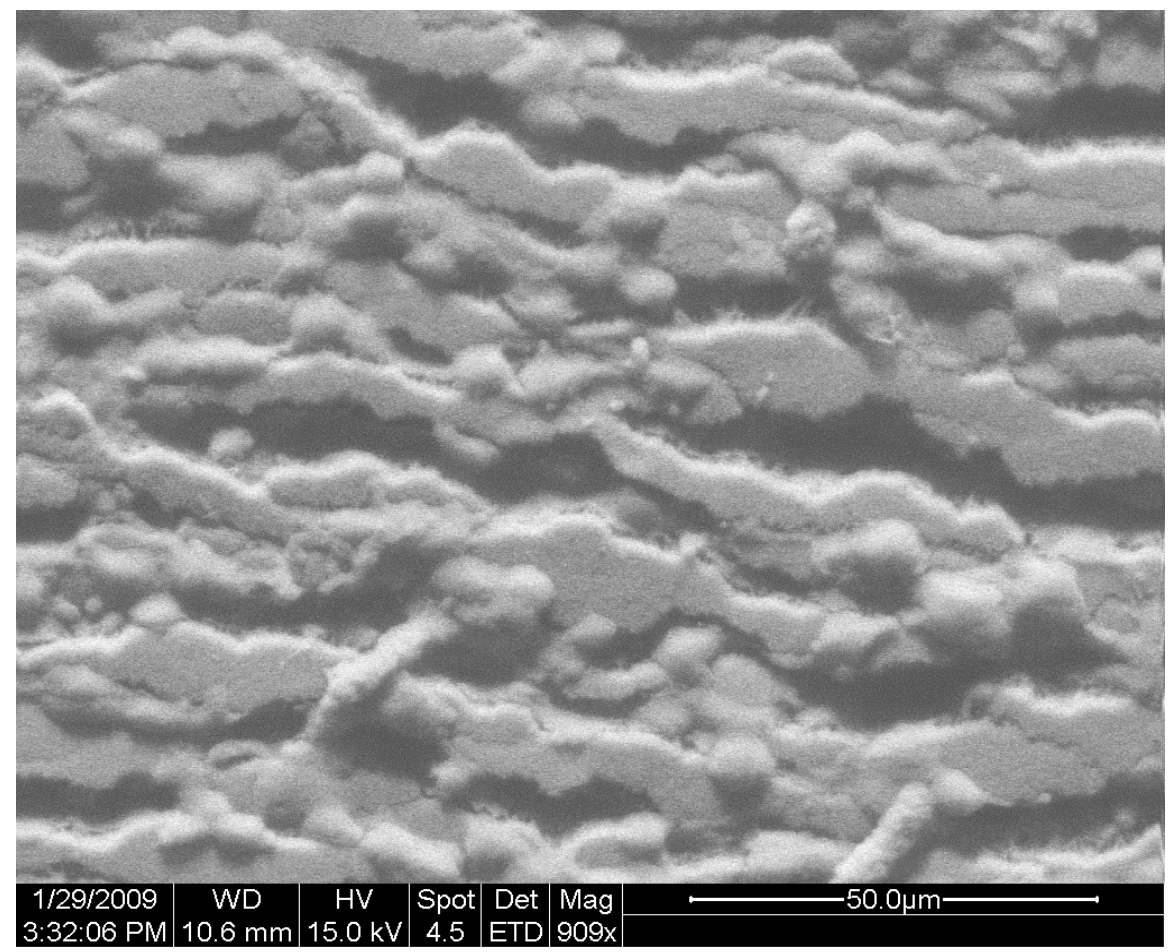

Figure A.10 Higher magnification SEM image of construct PB5 (72 hours of steady flow on 8-roller pump @ 15ml/min and 24 hours of pulsatile flow on 3-roller pump at $18.5 \mathrm{ml} / \mathrm{min}$ and $\sim 80 \mathrm{mmHg}$ ). 


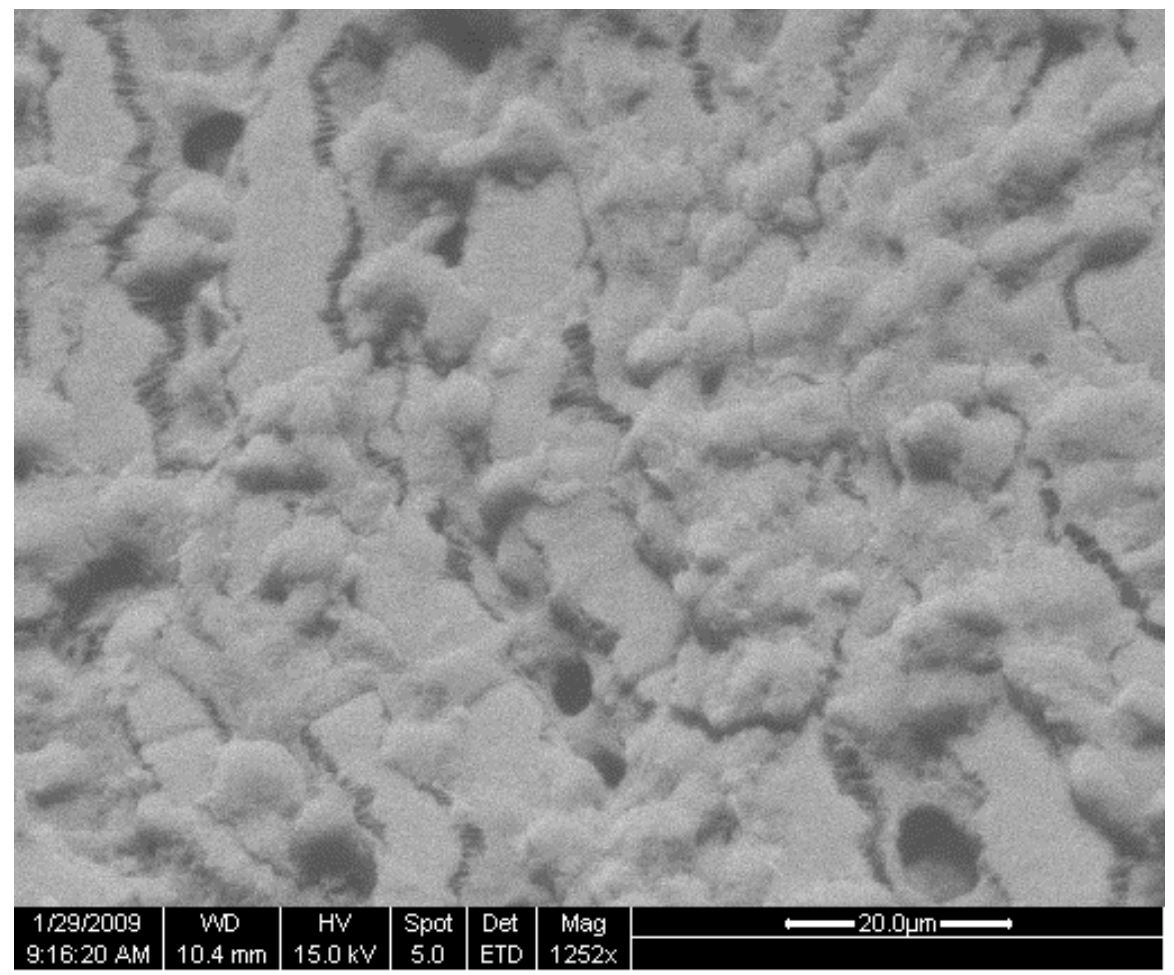

Figure A.11 Higher magnification SEM image of construct SA1 (0\% Dextran, $15 \mathrm{ml} / \mathrm{min} 0.3592 \mathrm{dyn} / \mathrm{cm} 2$ for 24 hours).

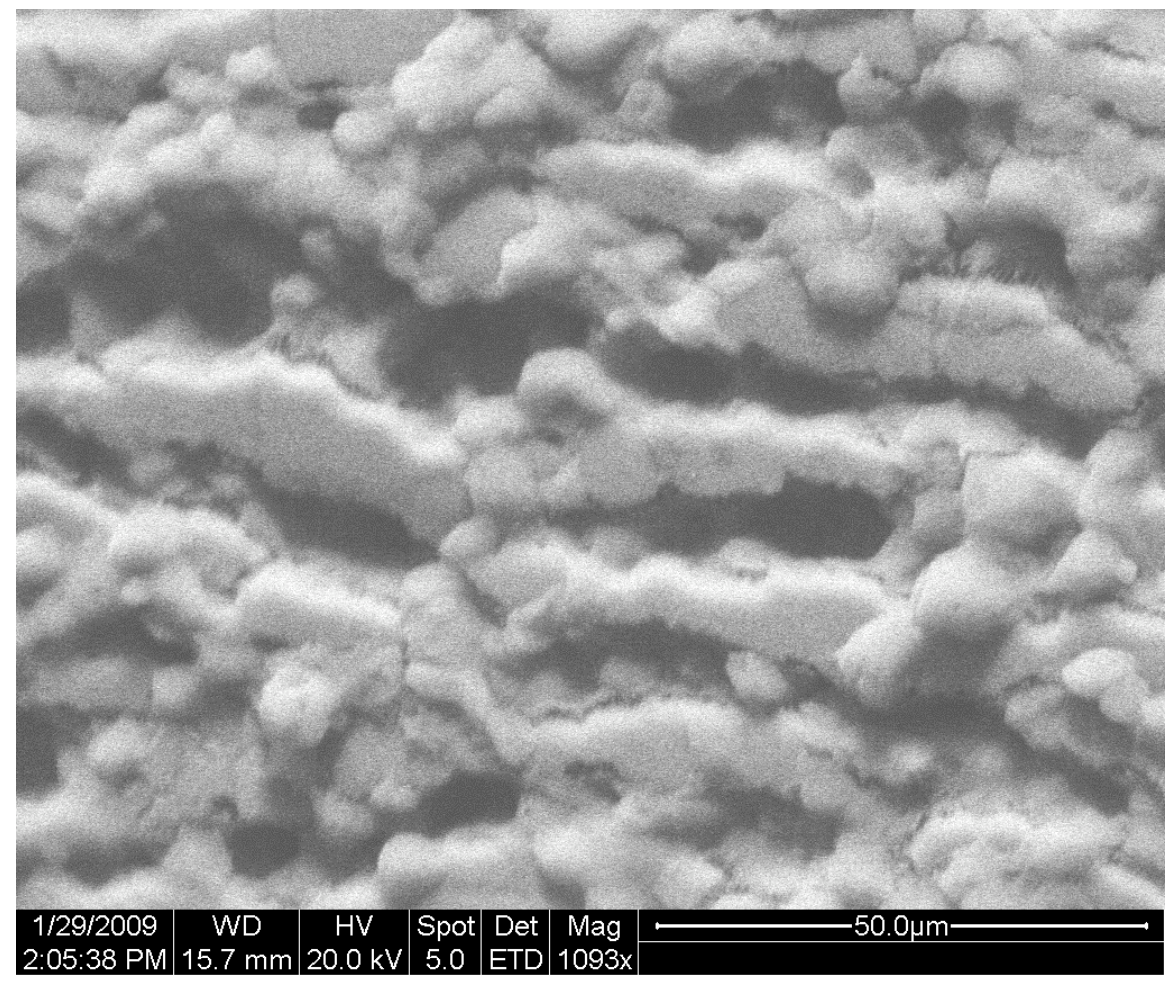

Figure A.12 Higher magnification SEM image of construct SB2 (14\% Dextran, $28 \mathrm{ml} / \mathrm{min} 6.3982 \mathrm{dyn} / \mathrm{cm} 2$ for 24 hours). 


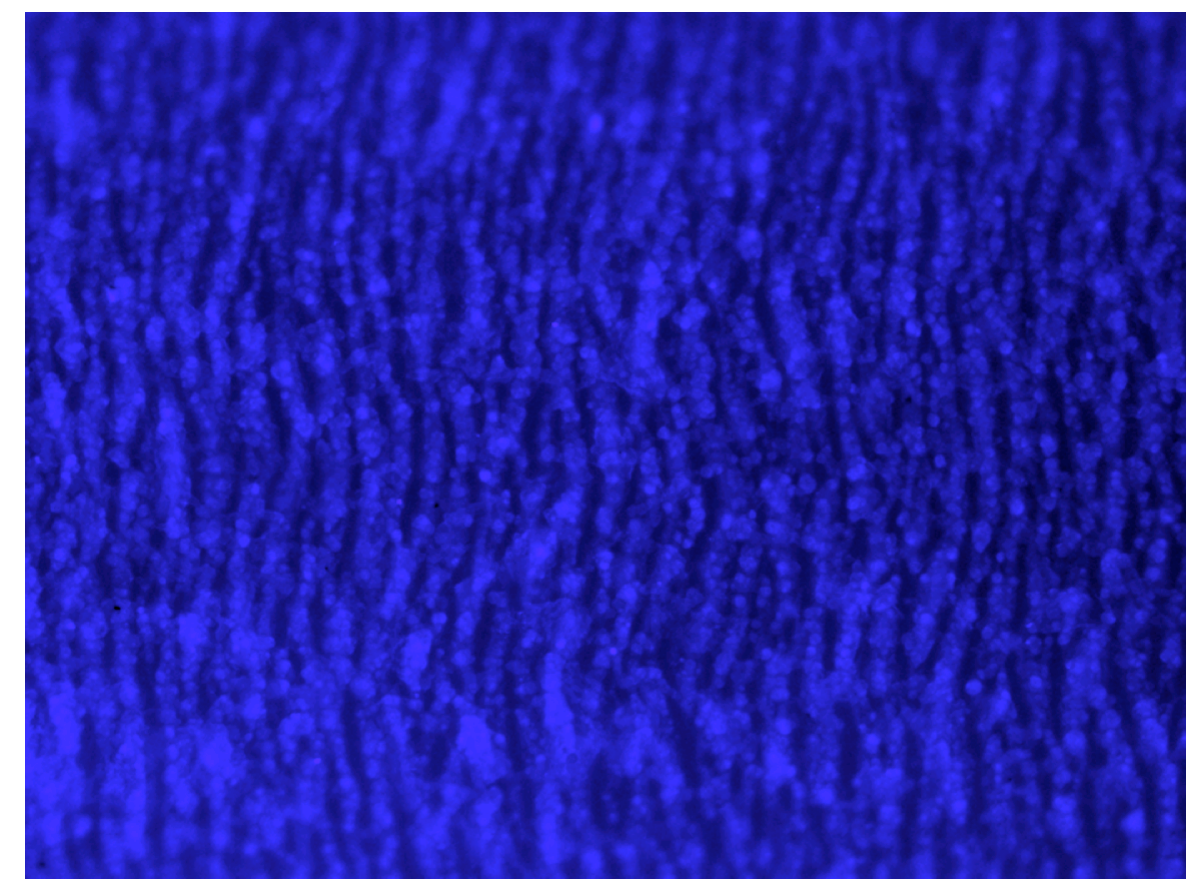

Figure A.13 BBI image of construct SA3 (14\% Dextran, 15ml/min, $3.4251 \mathrm{dyn} / \mathrm{cm} 2$ for 24 hours).

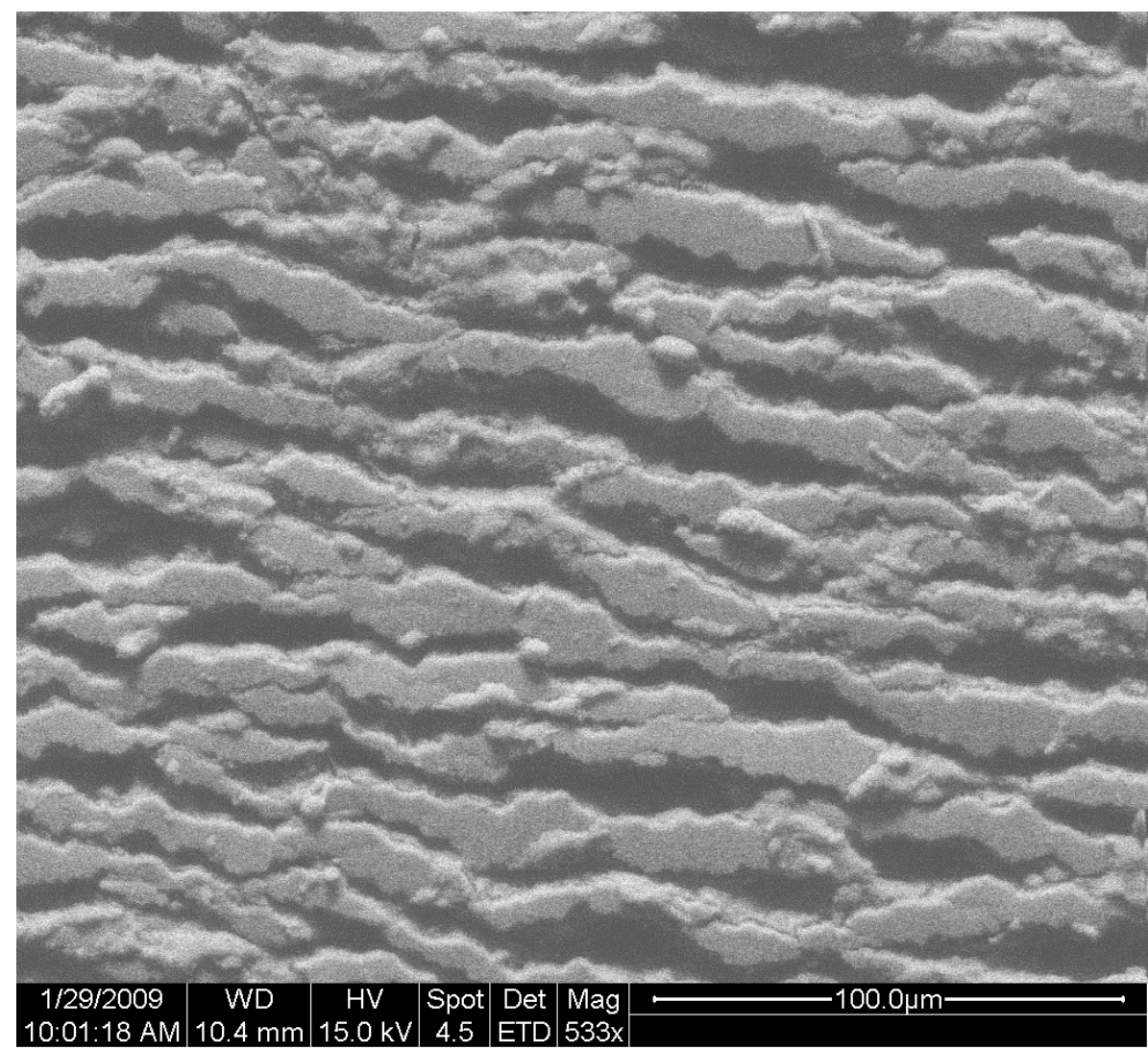

Figure A.14 SEM image of construct SA3 (14\% Dextran, 15ml/min, $3.4251 \mathrm{dyn} / \mathrm{cm} 2$ for 24 hours). 


\section{APPENDIX B: Procedural Protocols}

\section{LABCHART PRESSURE ACQUISITION SYSTEM}

\section{Initiating the System}

Turn on the power to the PC, AD Instruments Input Amplifier and Bridge Amp

Start the LabChart software.

Open the Input Amplifier window.

Select an appropriate Range depending on the current input voltage $(10 \mathrm{mV})$

Shift and stretch the vertical amplitude axis to make the best use of the available display area

Leave all other settings as the default

Press $O K$

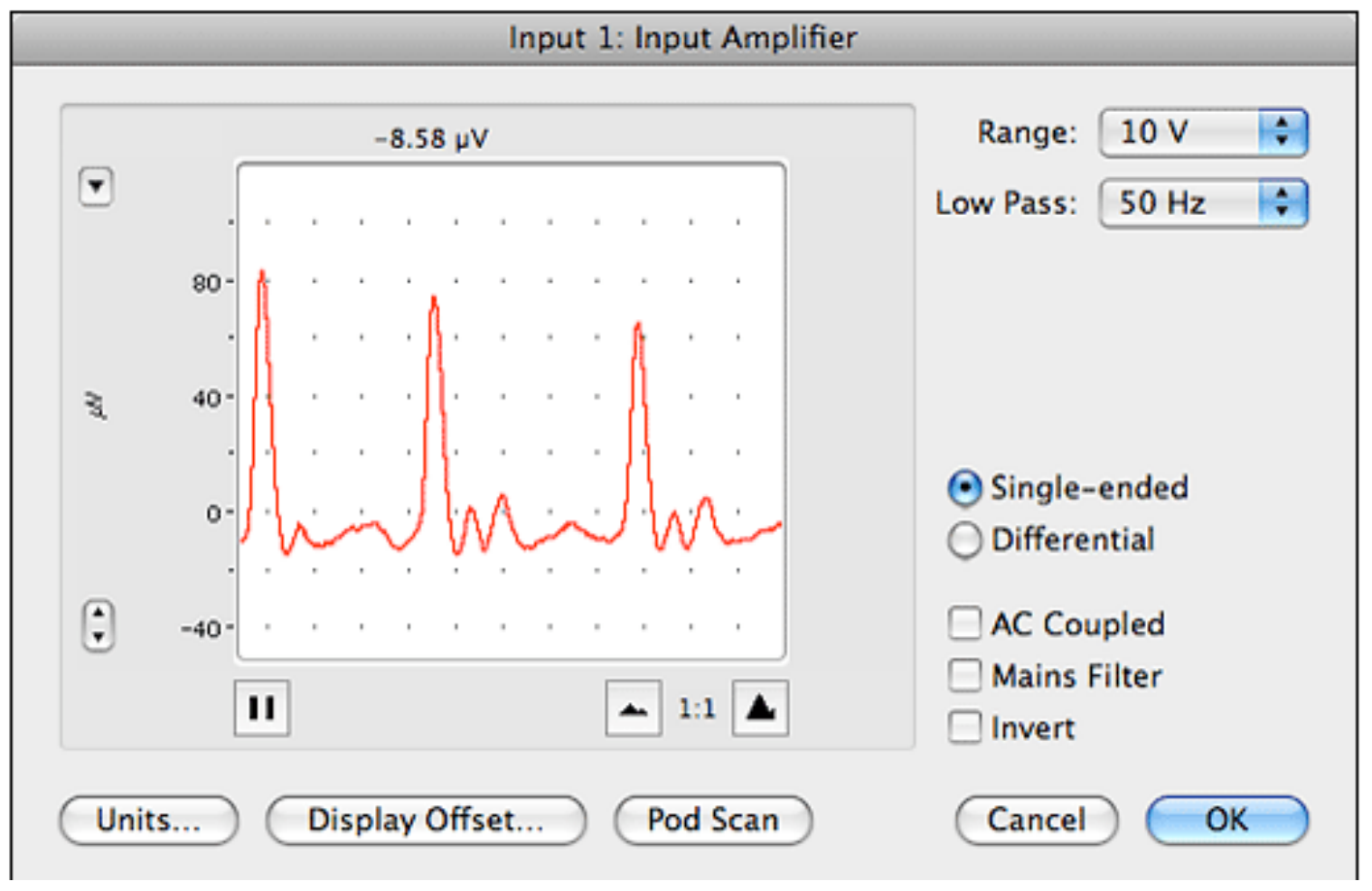




\section{$\underline{\text { Units Conversion/Calibration }}$}

Open the Units Conversion window by selecting the Channel 1 Drop box

Ensure that "2-point Calibration" is displayed in the drop box at the top of the window.

Convert the input voltage signal of channel 1 to "mmHg" using the Unit drop box.

For Point 1: enter the current input voltage in the left field and $0 \mathrm{mmHg}$ in the right field.

Connect the mercury manometer to the Pressure Transducer.

Pump the manometer up to $200 \mathrm{mmHg}$

For Point 2: enter the new input voltage in the left field and $200 \mathrm{mmHg}$ in the right field

Press $O K$

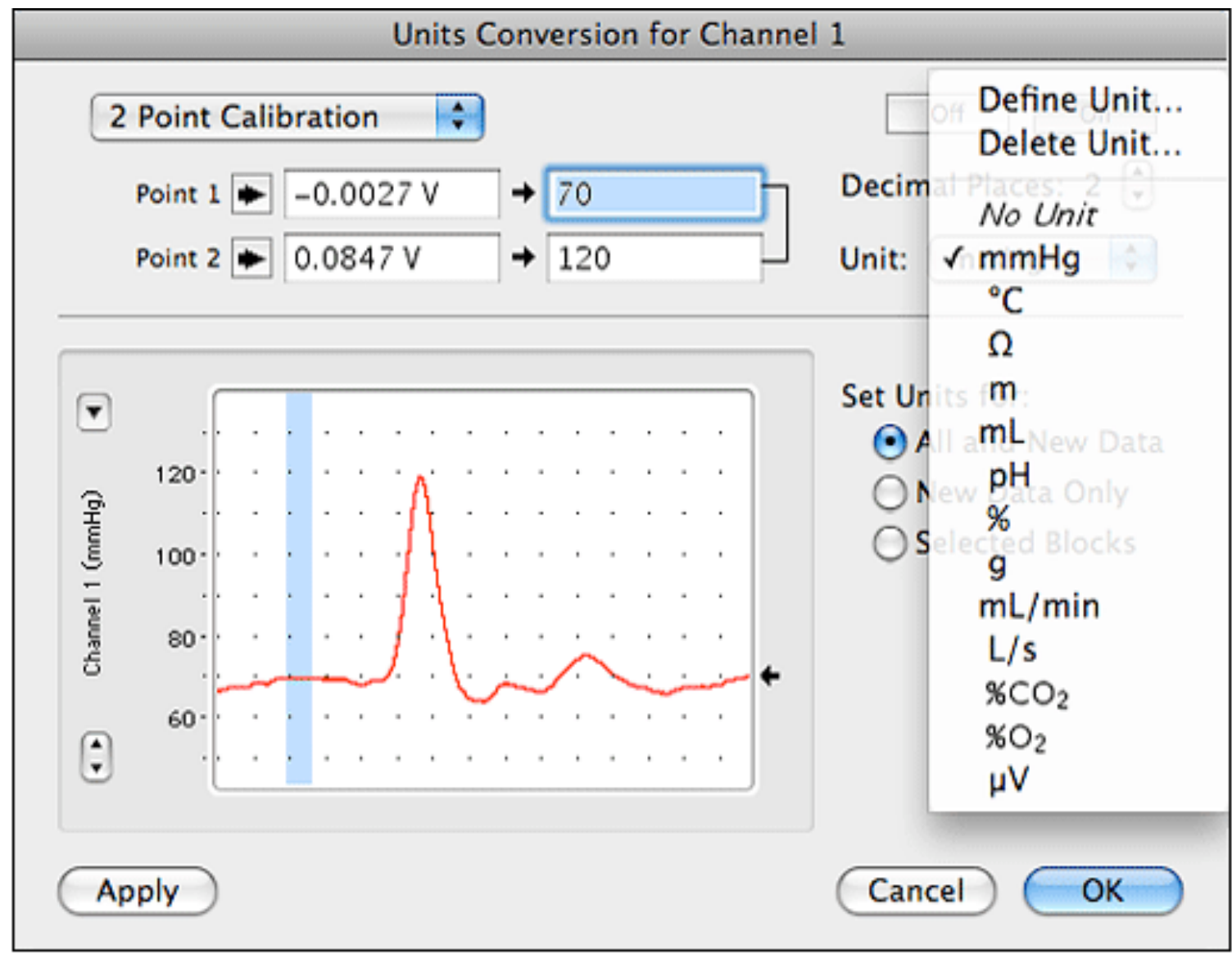




\section{Channel Settings}

In the Setup Menu select "Channel Settings..."

Change the Number of Channels to " 3 "

Press $O K$

Select "Cyclic Variables" in the Channel 2 drop box

Change the Source to "Channel 1"

Ensure that "Rate meter" is displayed in the second drop box

Press $O K$

Select "Arithmetic" in the Channel 3 drop box

Enter "Ch $2 / 3.25$ " in the Channel $3=$ dialog box

Press $O K$

\section{Cycle Variables}

Cycle Variables for Channel 2

Source: Channel 1 $†$ Ratemeter $\quad$ Default Scale: 2000 BPM $\quad$

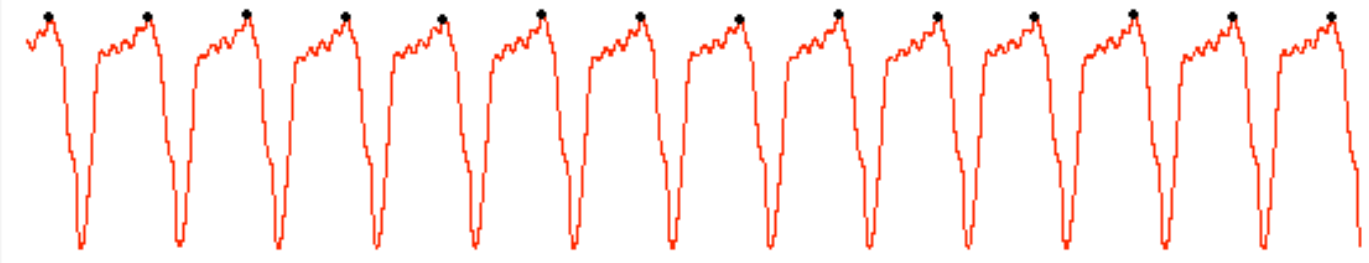

$10.840 \mathrm{~s}$

Displayed Data

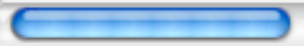

Noise Threshold (\%)

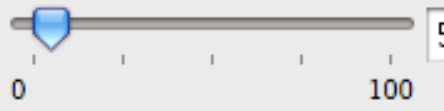

Save As Text...
Trigger:

$\odot$ Maximum

Minimum
Tracking: Off $\bullet$

Average: 2

\section{Taking Measurements and Recording Pressure}

The system is now ready to record system pressure. 
Start pump, press the Start button in the upper right corner of the window.

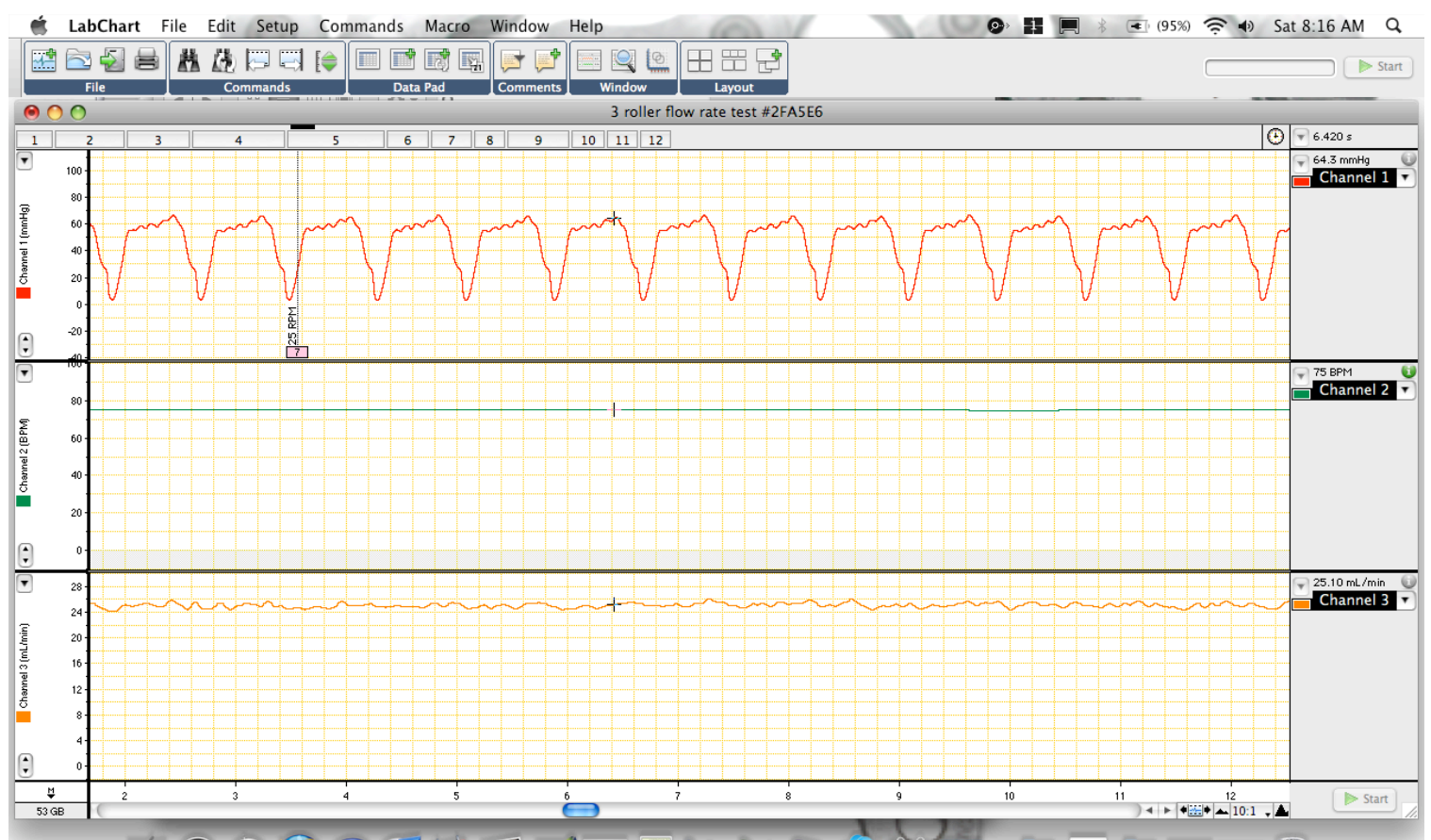




\section{OPERATION OF THE VILASTIC-3 VISCOMETER}

\section{STARTUP}

Turn on Vilastic-3 viscometer and allow it to warm up for at least 30 minutes before analyzing samples.

Turn on PC and Start VMax Software.

Ensure that the correct sample tube is selected on the home screen.

\section{SAMPLE FILLING}

Obtain an appropriate sample cup.

The standard sample cups (red) require $1 \mathrm{ml}$ of sample when using the $1 \mathrm{~mm}$ i.d. measurement tube or up to $1.5 \mathrm{ml}$ of sample with the $2 \mathrm{~mm}$ i.d. tube.

The small sample cups (clear) can be used for smaller sample volumes with only the $1 \mathrm{~mm}$ i.d. measurement tube.

Place the sample cup into the sample cup holder of the measurement tube assembly.

Using the standard sample cup, fill the cup with $\sim 1 \mathrm{ml}$ of sample. Take care not to inject any air bubbles into the sample.

Thread the sample cup holder onto the bottom of the measurement tube.

To begin the sample loading procedure, rotate the stopcock lever to the fill position.

Pull up on the syringe approximately $0.010 \mathrm{ml}$.

This will form an air slug in the measurement tube that during the filling procedure will sweep the tube clear of residual water.

Return the stopcock to the discharge position.

Insert the thermistor into the hole located on the measurement tube.

Move the stopcock lever to the fill position and slowly draw up on the syringe.

The sample should move through the tube, pushing the air slug into the clear chamber of the tube.

NOTE If the air bubble does not release from the top of the tube or attaches to the walls of the acrylic reservoir, the tube and system should be thoroughly cleaned to remove contaminants.

Continue drawing up with the syringe until this air slug moves above and beyond the stopcock. This should leave a water/sample interface in the clear chamber of the tube.

This will take approximately $0.25 \mathrm{ml}$ of sample.

If you are using the red cups and have placed at least $1 \mathrm{ml}$ of sample in the cup continue to draw-up on the syringe until $0.4 \mathrm{mls}$ have been drawn. 
Return the stopcock to the discharge position. The tube is now filled and ready for measurement.

CAUTION: PRESSURE SENSOR LIMITS If the pressure in the measurement system exceeds $40 \mathrm{psi}$, the pressure sensor may be damaged. Be very careful during the filling and purging operation not to produce excessive pressure that could damage the pressure sensor. During measurements the pressure will not exceed $\sim 1 \mathrm{psi}$; if the measurement tube is blocked then it is possible to develop extreme pressures when filling the measurement tube that could damage the sensor.

DENSITY For accurate calculations, the density of a test sample should be known to within $1 \%$ or better accuracy. The sensitivity of measured values to density can be determined by modifying the density value a small amount and recalculating the data. To enter the density select Set Up menu option and the Density submenu option.

\section{SAMPLE EVALUATION}

Perform a quick evaluation of the test sample at two operator-selected drive levels to determine the possible measurement range of shear rate or strain for a given fluid sample.

When testing 0-30\% dextran media, use the following settings.

Enter " 2 " in the Frequency box

Enter "5" in the Integration Time box

Enter "10" in the Low Drive box

Enter "30" in the High Drive box

Press Evaluate Sample 


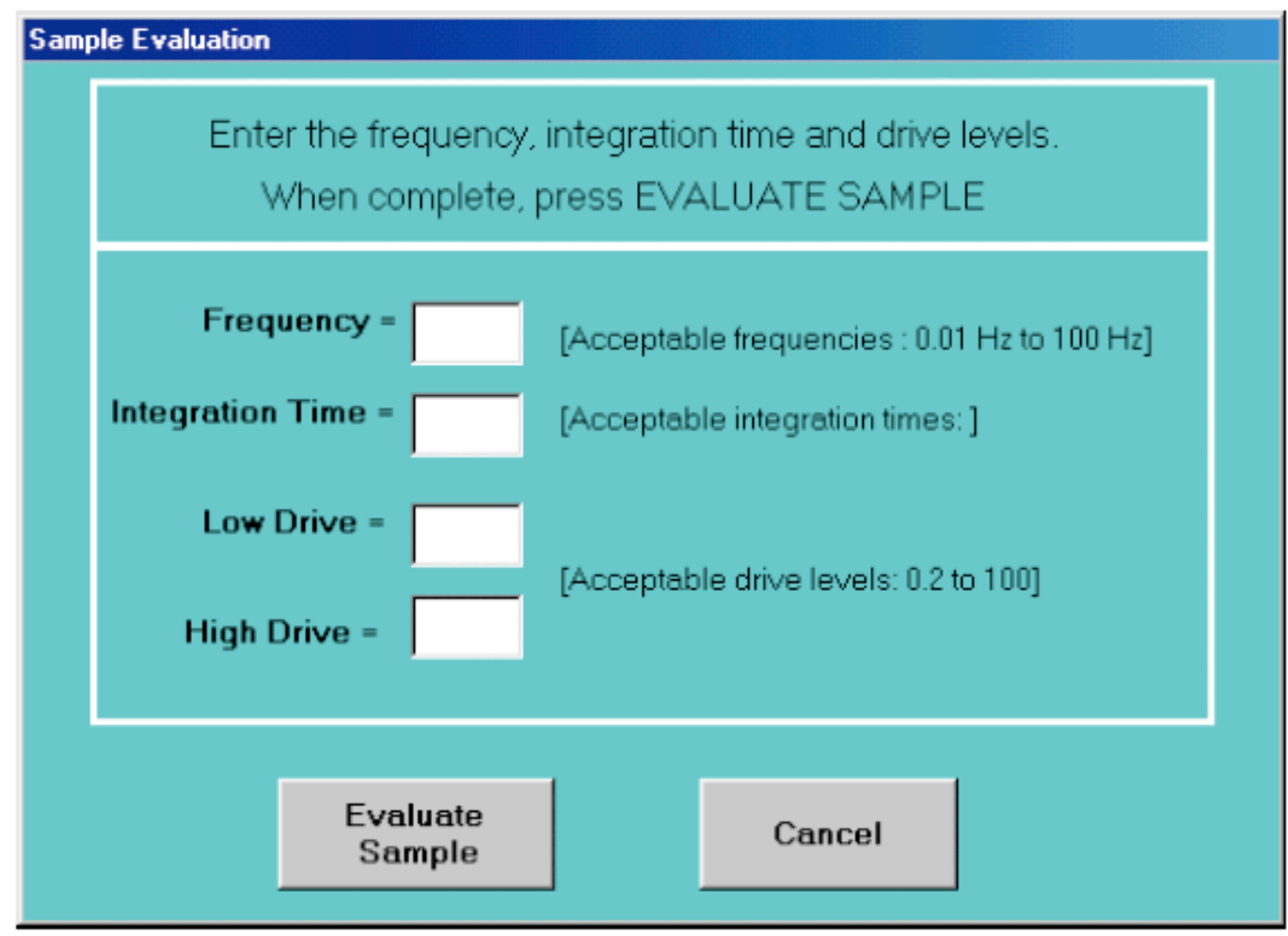

After measurements are completed diagnostics will appear on screen. The diagnostics indicate if there are any questionable measurements. After the display of diagnostics, the measured properties of the fluid at the two drive levels are displayed. The results are used by the software to determine the minimum and maximum shear rate or strain possible.

\section{SET PROTOCOL}

After a sample evaluation has been completed the option to set a sequence of shear rate or shear strain measurements is available.

Select Set Protocol under the Protocols menu to set the parameters of the run.

The frequency of measurement is fixed at the frequency of the sample evaluation.

Ensure that the integration time is set to $5 \mathrm{sec}$.

Select "Shear Rate" in the measurement sweep options

The acceptable range of the sweep parameter was determined by the sample evaluation.

Enter a minimum and maximum shear rate within this range.

If the sample evaluation did not produce the desired range, repeating the sample evaluation at other drive levels or Drive Switch position can produce different sweep parameter range. 
Enter the number of measurements desired.

Leave all other setting at the default selection

\section{Press Continue}

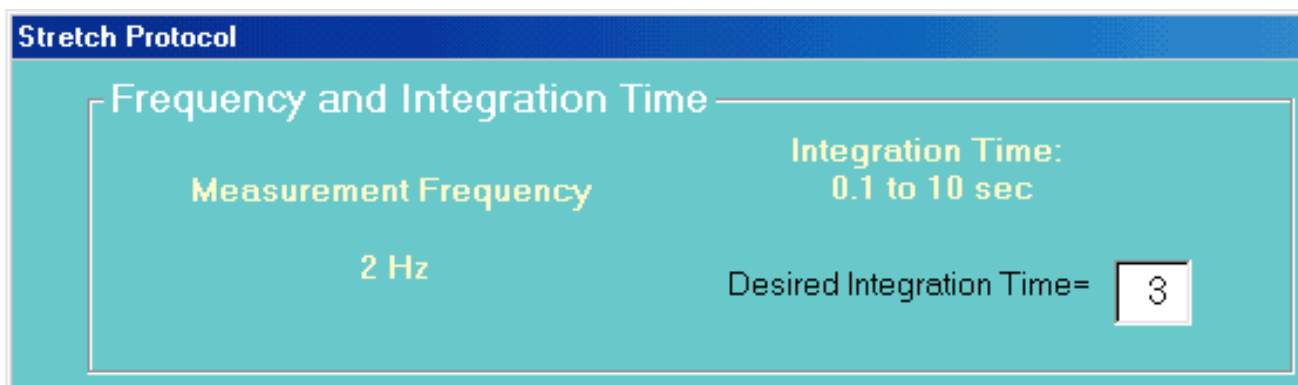

- Sweep Parameter

c Shear Strain

Shear Strain Range : 0.1052 to 105.5264

C Shear Rate

C Drive Level

Min.

Shear Strain =
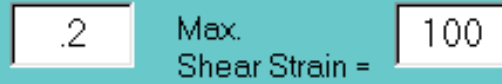

Measurement Protocol Interval Spacing:

cogarithmically Spaced

Measurements

c Linearly Spaced

Measurements

Sequence:

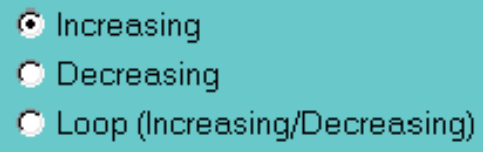

Number of Measurements: 2 to 200

Number of Measurements $=\quad 2$

Continue

Cancel

\section{MEASUREMENTS}

Once a STRETCH protocol or previous protocol has been established to initiate measurements;

Select the menu option Run/Calculate.

Select Measure and Calculate.

Click Start $\boldsymbol{R} \boldsymbol{U N}$ 
The viscosity vs. shear rate plot will be displayed during the measurements.

When all measurements are complete, the resulting data will be displayed and can be viewed in text and graphical formats. 
Date

Study Information

Study Title:

Purpose:

Material:

Diameter

Length:

Cell Type:

\section{Prep 1 week prior}

1. Gas sterilize biochambers and 2-port reservoirs

2. Determine target number of cells and passage schedule, then thaw cells

3. Cut grafts, mount on fittings, and suture

4. Autoclave grafts, flasks, and forceps

Prep the day before

5. Make media:

a. Bioreactor Media (Human Complete w/o ECGS w/ antibiotics)

b. Conditioning Media (1:6 solution of FBS:M199 + antibiotics)

6. Denucleate grafts (using filtered $\mathrm{EtOH}$ )

a. $15 \min 70 \% \mathrm{EtOH}$

b. $15 \mathrm{~min} 100 \% \mathrm{EtOH}$

c. Leave in degassed Conditioning Media in incubator overnight

Set-up day: BVM conditioning

7. Warm up media (Bioreactor Media and Conditioning Media)

8. Insert sterile grafts into biochambers ** requires non-sterile partner**

9. Using $60 \mathrm{~mL}$ syringe, flush lumen with Conditioning Media to prime graft

a. Clamp lumen and continue to prime graft and chamber until chamber is full

b. Repeat for all vessels

10. Place small WM pump in hood

11. Prime 2-port reservoirs with Conditioning Media

12. Attach primed biochamber to 2-port reservoir and condition graft for $10 \mathrm{~min}$

a. Flow through lumen first to remove air, then clamp lumen and condition transmurally on $90 \mathrm{rpm}$ setting

13. Leave primed biochambers in large incubator until ready for sodding step

14. Prime 2-port reservoirs with Bioreactor Media

a. Prepare one for each vessel

b. Be sure that drip is visible and outlet is submerged

c. Clamp tubing and leave in big incubator

Set-up day: BVM sodding

15. Take corresponding number of primed biochambers and reservoirs to hood
Initials

16. Attach outlet of reservoir to inlet stopcock of biochamber

a. Leave reservoir inlet unattached and biochamber outlet facing trough

17. Record BVM numbers:

18. Harvest cells

a. Apply Trypsin, deactivate with media

b. Take $100 \mathrm{uL}$ from total $\mathrm{mL}$ cells Counts:

19. $\mathrm{X}=$

x $2000 \times$ (cell mL x .10)

total number of cells $=$

20. Pellet cell suspension (on 4 for $4 \mathrm{~min}$ )

21. Resuspend in $\mathrm{mL}$ Bioreactor Media

22. Sod each graft with ___ $\mathrm{mL}$ cell solution

a. Cells per graft $=$

b. Sodding density $=\quad$ cell $\mathrm{s} / \mathrm{cm} 2$

23. Chase with $1-3 \mathrm{~mL}$ Bioreactor Media

24. Attach biochamber outlet to reservoir inlet

25. Bring BVMs to large incubator

26. Place on small WM pump - leave lumen clamped!!

27. Immediately begin transmural flow at $7 \mathrm{rpm}$, and maintain for 1 hour

a. Started on pump at: ___ (time)

28. Unclamp lumen and maintain $7 \mathrm{rpm}$ luminal flow for 1 hour

29. Increase flow to $11 \mathrm{rpm}$

30. Increase flow to $15 \mathrm{rpm}$; leave overnight

The next day and beyond

31. Increase flow by $10-15 \mathrm{rpm}$ at a time to reach $90 \mathrm{rpm}$ by the end of the day

32. BVM maintenance: replace media reservoirs every $3^{\text {rd }}$ day

a. Check CO2!

BVM Usage

Stented:

OCT Imaged:

Other:

BVM Analysis

BBI:

SEM:

Histology/Stains: 
APPENDIX C: Trial Construct Location

\begin{tabular}{|c|c|c|c|}
\hline Block \# & $\begin{array}{c}\text { Sample } \\
\text { Description }\end{array}$ & $\begin{array}{c}\text { Sample } \\
\text { Orientation }\end{array}$ & $\begin{array}{c}\text { Date } \\
\text { Embedded }\end{array}$ \\
\hline $09-019$ & SB3 & Cross & $4 / 14 / 09$ \\
\hline $09-020$ & SB2 & Cross & $4 / 14 / 09$ \\
\hline $09-021$ & SB1 & Cross & $4 / 14 / 09$ \\
\hline $09-022$ & SA2 & Cross & $4 / 14 / 09$ \\
\hline $09-023$ & SA3 & Cross & $4 / 14 / 09$ \\
\hline $09-024$ & SA1 & Cross & $4 / 14 / 09$ \\
\hline $09-025$ & PB5 & Cross & $4 / 14 / 09$ \\
\hline $09-026$ & PB4 & Cross & $4 / 14 / 09$ \\
\hline $09-027$ & PB3 & Cross & $4 / 14 / 09$ \\
\hline $09-028$ & PB1 & Cross & $4 / 14 / 09$ \\
\hline $09-029$ & PB2 & Cross & $4 / 14 / 09$ \\
\hline $09-030$ & PA1 & Cross & $4 / 14 / 09$ \\
\hline
\end{tabular}




\section{APPENDIX D: Figure Reprint Permissions}

\section{Usage Agreement - Encyclopedia Britannica}

\section{Terms of Use}

These Terms of Use govern your use of Encyclopædia Britannica ${ }^{\circledR}$ Online and, unless other terms and conditions expressly govern, any other electronic services provided by Encyclopædia Britannica, Inc. ("Britannica") that may be available from time to time (collectively, the "Services").

General: Your use of the Services constitutes your agreement to these Terms of Use. If you do not agree with these Terms of Use, please do not use the Services. Britannica reserves the right to change, modify, add, or remove portions of these Terms of Use at any time. Please check this page periodically for any modifications. Your use of any of the Services following the posting of changes constitutes your acceptance of the changes.

Ownership: The content on the Services is the property of Britannica, its affiliated companies or licensors and is protected by international copyright, patent, and trademark laws.

Advertising:Advertisements, promotions, and marketing messages may appear on the Services from time to time.

Use of Content: You may display, reproduce, print or download content on the Services only for your personal, non-commercial use. If you are a teacher, scholar or student, you may copy reasonable portions of the content for lesson plans, interactive whiteboards, reports, dissertations, presentations, school newspapers and for similar nonprofit educational purposes to the extent permitted by applicable law. In each case, however, you may not remove or alter any copyright, trademark, service mark or other proprietary notices or legends. You may not publish, distribute, retransmit, sell or provide access to the content on the Services, except as permitted under applicable law or as described in these Terms of Use. Britannica works to ensure that all the content on its Services is in compliance with applicable U.S. copyright laws. However, in the case of works on the Services authored by parties other than Britannica, you may wish to check on their copyright status before downloading them if you are in another country.

If you want to reproduce or use content for any purpose or in any manner other than as described above, you will need Britannica's permission. Requests should be directed to this syndication form. 


\begin{tabular}{|c|c|c|}
\hline License Number & \multicolumn{2}{|c|}{2175060152052} \\
\hline License date & \multicolumn{2}{|c|}{ Apr 23, 2009} \\
\hline $\begin{array}{l}\text { Licensed content } \\
\text { publisher }\end{array}$ & \multicolumn{2}{|c|}{ Springer } \\
\hline $\begin{array}{l}\text { Licensed content } \\
\text { publication }\end{array}$ & \multicolumn{2}{|c|}{ Annals of Biomedical Engineering } \\
\hline Licensed content title & \multicolumn{2}{|r|}{$\begin{array}{l}\text { Physiologic Pulsatile Flow Bioreactor Conditioning of Poly(ethylene glycol)-based Tissue } \\
\text { Engineered Vascular Grafts }\end{array}$} \\
\hline Licensed content author $\mathrm{M}$ & \multicolumn{2}{|r|}{ Mariah S. Hahn } \\
\hline Licensed content date $\mathrm{F}$ & \multicolumn{2}{|c|}{ Feb 1, 2007} \\
\hline Volume number & \multicolumn{2}{|c|}{35} \\
\hline Issue number & \multicolumn{2}{|c|}{2} \\
\hline Type of Use & \multicolumn{2}{|c|}{ Thesis/Dissertation } \\
\hline Portion & \multicolumn{2}{|c|}{ Figures } \\
\hline $\begin{array}{l}\text { Title of your thesis / } \\
\text { dissertation }\end{array}$ & \multicolumn{2}{|r|}{$\begin{array}{l}\text { IMPLEMENTATION OF PHYSIOLOGIC FLOW CONDITIONS IN A BLOOD VESSEL MIMIC } \\
\text { BIOREACTOR SYSTEM FOR THE EVALUATION OF INTRAVASCULAR DEVICES }\end{array}$} \\
\hline Estimated size(pages) & \multicolumn{2}{|c|}{158} \\
\hline Total & \multicolumn{2}{|c|}{0.00 USD } \\
\hline License Number & & 2175061030310 \\
\hline License date & & Apr 23, 2009 \\
\hline \multicolumn{3}{|l|}{ Licensed Chapter Title } \\
\hline \multicolumn{3}{|l|}{ Licensed Chapter ID } \\
\hline Licensed content publisher & & Elsevier \\
\hline Licensed content publicatio & & European Journal of Cardio-Thoracic Surgery \\
\hline Licensed content title & & Tissue engineering of small caliber vascular grafts \\
\hline Licensed content author & & $\begin{array}{l}\text { Simon P. Hoerstrup, Gregor Zünd, Ralf Sodian, Andrea M. Schnell, Jürg Grünenfelder and } \\
\text { Marko I. Turina }\end{array}$ \\
\hline Licensed content date & & July 2001 \\
\hline Volume number & & 20 \\
\hline Issue number & & 1 \\
\hline Pages & & 6 \\
\hline Type of Use & & Thesis / Dissertation \\
\hline Portion & & Figures/table/illustration/abstracts \\
\hline Portion Quantity & & 1 \\
\hline Format & & Both print and electronic \\
\hline $\begin{array}{l}\text { You are the author of this } \\
\text { Elsevier article }\end{array}$ & & No \\
\hline Are you translating? & & No \\
\hline \multicolumn{3}{|l|}{ Order Reference Number } \\
\hline Expected publication date & & Apr 2009 \\
\hline Elsevier VAT number & & GB 494627212 \\
\hline Billing type & & Invoice \\
\hline Company & & Marc C Dawson \\
\hline \multirow{3}{*}{\multicolumn{2}{|c|}{ Billing address }} & 1401 Chorro St. \\
\hline & & San Luis Obispo, CA 93401 \\
\hline & & United States \\
\hline \multicolumn{3}{|l|}{ Customer reference info } \\
\hline Permissions price & & 0.00 USD \\
\hline Value added tax $0.0 \%$ & & 0.00 USD \\
\hline Total & & 0.00 USD \\
\hline
\end{tabular}




\begin{tabular}{|c|c|}
\hline License Number & 2175061265242 \\
\hline License date & Apr 23, 2009 \\
\hline \multicolumn{2}{|l|}{ Licensed Chapter Title } \\
\hline \multicolumn{2}{|l|}{ Licensed Chapter ID } \\
\hline Licensed content publisher & Elsevier \\
\hline Licensed content publication & Medical Engineering \& Physics \\
\hline Licensed content title & $\begin{array}{l}\text { The effect of dynamic culture conditions on endothelial cell seeding and retention on small } \\
\text { diameter polyurethane vascular grafts }\end{array}$ \\
\hline Licensed content author & Shan-hui Hsu, I-jine Tsai, Da-jun Lin and David C. Chen \\
\hline Licensed content date & April 2005 \\
\hline Volume number & 27 \\
\hline Issue number & 3 \\
\hline Pages & 6 \\
\hline Type of Use & Thesis / Dissertation \\
\hline Portion & Figures/table/illustration/abstracts \\
\hline Portion Quantity & 1 \\
\hline Format & Both print and electronic \\
\hline $\begin{array}{l}\text { You are the author of this } \\
\text { Elsevier article }\end{array}$ & No \\
\hline Are you translating? & No \\
\hline \multicolumn{2}{|l|}{ Order Reference Number } \\
\hline Expected publication date & Apr 2009 \\
\hline Elsevier VAT number & GB 494627212 \\
\hline Billing type & Invoice \\
\hline Company & Marc C Dawson \\
\hline \multirow[t]{3}{*}{ Billing address } & 1401 Chorro St. \\
\hline & San Luis Obispo, CA 93401 \\
\hline & United States \\
\hline \multicolumn{2}{|l|}{ Customer reference info } \\
\hline Permissions price & 0.00 USD \\
\hline Value added $\operatorname{tax} 0.0 \%$ & 0.00 USD \\
\hline Total & 0.00 USD \\
\hline
\end{tabular}

\section{Dear Marc Dawson}

Thank you for your email request. Permission is granted for you to use the material below for your thesis/dissertation subject to the usual acknowledgements and on the understanding that you will reapply for permission if you wish to distribute or publish your thesis/dissertation commercially.

Kind Regards,

Katie B Wade

Permissions Assistant

Wiley-Blackwell

9600 Garsington Road

Oxford OX4 2DQ

UK

Tel: $+44(0) 1865476149$

Fax: +44 (0) 1865471158

Email: katie.wade@wiley.com 
-----Original Message-----

From: Marc Dawson [mailto:mcdawson78@hotmail.com]

Sent: 24 April 2009 02:11

To: Permission Requests - UK

Subject: Permission request

Hello,

I am a Master student at California Polytechnic State University in San Luis Obispo, CA and I would like to use a figure from one of your publications in my Masters Thesis.

The Article:

Pulse wave analysis. British journal of clinical pharmacology, 2001. 51(6)

Authored by:

O'Rourke, M.F., A. Pauca, and X.J. Jiang

Figure 1, page 509

My paper will be available to university students and faculty only. Not public.

Thank you,

Marc C. Dawson 


\section{AMA AMERICAN \\ ASSOCIATION}

JOURNAL PERMISSIONS REQUEST

May 9, 2009

\begin{tabular}{lllll}
\hline Marc & Dawson & Client\# & 14636 \\
P.O. Box 15014 & & & Request\# & 23374 \\
$\begin{array}{l}\text { San Luis Obispo } \\
\text { USA }\end{array}$ & CA & 93406 & \\
\hline \hline
\end{tabular}

In response to your request to use:

$\begin{array}{lccc}\text { Journal Name } & \text { Year } & \text { Citation } & \text { Item used } \\ \text { JAMA } & 1999 & 282(21): 2035-2042 & \text { Figure 2 }\end{array}$

Intended Use: Reproduction for use in Masters Thesis "IMPLEMENTATION OF PHYSIOLOGIC FLOW CONDITIONS IN A BLOOD VESSEL MIMIC BIOREACTOR SYSTEM FOR THE EVALUATION OF INTRAVASCULAR DEVICES' ${ }^{-1}$ for presentation at Cal Poly State University, San Luis Obispo.

\section{PERMISSION GRANTED}

Thank you for your interest in AMA's scientific publications. Rights granted herein are non-exclusive for reproduction in print, online and CD-ROM as specified in this request. If the permission requested is for inclusion of AMA material in a book or CD-ROM, such permission is granted for the single edition only as specified in this request.

Your credit line must include the name of the publication, issue date, volume and page number, as well as "Copyright $\oplus$ (Year of Publication) American Medical Association. All rights reserved.

American Medical Association Journal Permissions

$515 \mathrm{~N}$. State St., $11^{\text {th }}$ Floor Chicago IL 60654

Tel. (312) 464-2513 Fax (312) 464-5834 email: permissions@ama-assn.org 